\title{
FAMILY COHESION AND FLEXIBILITY IN \\ EARLY EPISODE PSYCHOSIS
}

By

Maya Gupta

A Thesis Submitted to the Graduate Program in Psychology

in conformity with the requirements for the

Degree of Doctor of Philosophy

Queen's University

Kingston, Ontario, Canada

July 2015

Copyright ( Maya Gupta, 2015 


\begin{abstract}
Our understanding of treatment for psychotic disorders has improved considerably over the last 20 years. One area that has received international attention is the importance of early intervention for psychosis. Research suggests that family support promotes recovery and is a key component of any successful early intervention program. However, very little is known about familial interaction patterns in families that are coping with the unexpected onset of a psychotic illness and the factors that help to foster adaptive family functioning during this challenging time.

The purpose of this study was to understand the individual, caregiver, and family factors that are associated with healthy and adaptive family functioning in early episode psychosis and to explore differences in family interaction patterns between families with an individual with psychosis and healthy control families. This study employed two methodologies: (1) an online caregiver survey that examined relationships among various caregiver and family system variables such as caregiver appraisals, coping strategies, quality of life, perceived helpfulness of support systems, and family functioning and (2) an experimental laboratory-based study that examined the real time affective states and dynamic flexibility of family interactions in families with an individual with early psychosis and healthy control families.

The results of this project indicate that cognitive appraisals about the illness, its consequences, and one's caregiving role are important predictors of family functioning. Specifically, greater positive caregiver appraisals were associated with better family functioning. Family members of an individual with psychosis reported more family difficulties across dimensions of cohesion and flexibility; however in the laboratory-
\end{abstract}


based observation study, families with an individual with psychosis were equally flexible and diverse in their expression of affect, and had more predictable interactions compared to healthy control families. This is the first study to use state space grids to visually depict family interactions in real time, demonstrating its unique applications both for research and clinical intervention.

The findings from this study have important implications for the field of early intervention in psychosis and could inform relevant treatment targets for family-based interventions. 


\section{Acknowledgments}

I would like to take this opportunity to express my sincere gratitude to my supervisor, Dr. Christopher Bowie, whose excellent mentorship, expertise, and encouragement guided me through graduate school. Thank you for fostering my growth and creativity as a scientist and clinician, and for instilling me with a sense of adventure in research. I am also grateful to Drs. Tom Hollenstein and Patricia Minnes for their patience, guidance, and invaluable contributions to this project. Thank you to Dr. Stephanie McDermid Vaz, Dr. Sean Kidd, Kelly Robinson, and Pat Offer for your interest in my work and for your efforts in helping to spread word about this project. I am greatly indebted to Jessica Lougheed for her expertise and support, and to Tanya Tran for her help in getting this study off the ground. To Sarah Cassidy and Mariana BorsukGudz, I am so grateful for your unparalleled work ethic, and unwavering commitment and enthusiasm even during the most arduous of tasks.

I offer my warm gratitude to my fellow lab mates, Katherine, Michael, Mike, and Melissa for their academic and emotional support throughout the years. Thank you to all my lab members, past and present, for making the lab a fun and positive learning environment. I would also like to give special thanks to my dear friends Katherine, Emma, Jenn, and Laura for being by my side through every major milestone and for making graduate school not only bearable, but incredibly fun. I would also like to thank my family for raising me to value innovation, social responsibility, and compassion, values that inspired me to go to graduate school and gave me strength to see it through. Finally, to my husband Scott, thank you for your unconditional love and generosity, and for always nurturing my dreams. 


\section{Table of Contents}

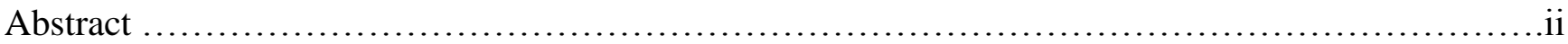

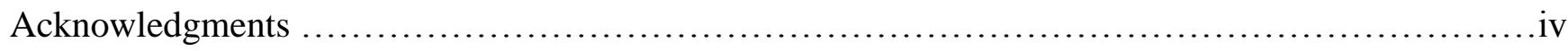

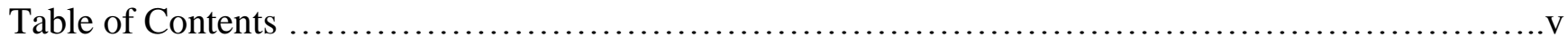

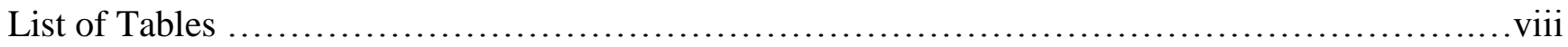

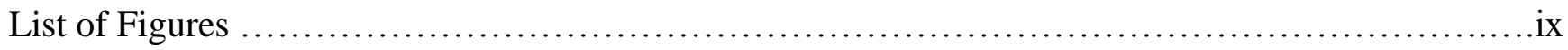

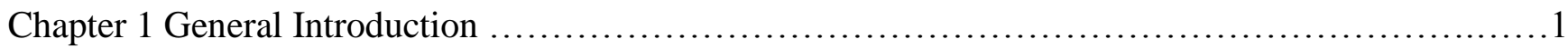

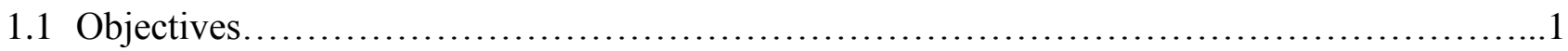

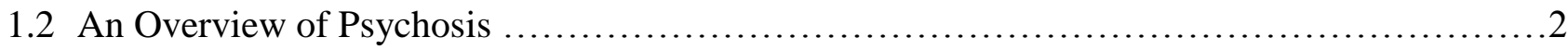

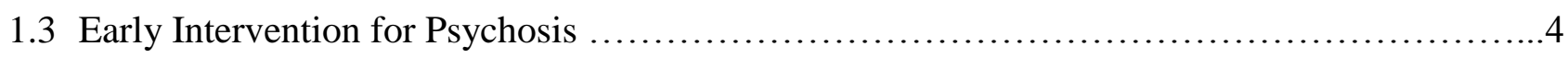

1.4 Family Functioning in Early Episode Psychosis …......................................

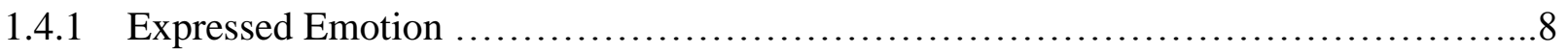

1.4.2 Family and Caregiver Burden .........................................................

1.4.3 Limitations and New Directions................................................

1.5 The Circumplex Model of Family Functioning............................................11

1.6 Dynamic Systems and State Space Grids ..................................................

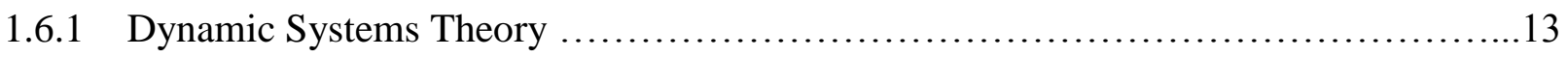

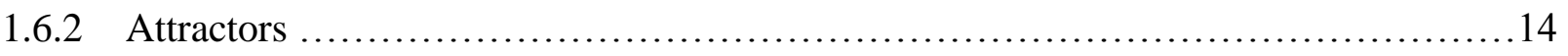

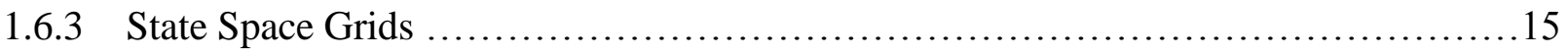

1.6.4 An Overview of Key State Space Grid Studies ......................................19

1.7 Towards a Better Understanding of Family Functioning in Early Psychosis ...................21

Chapter 2 Caregiver Appraisals and Family Functioning in Early Episode Psychosis..................24

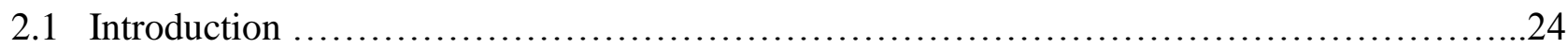




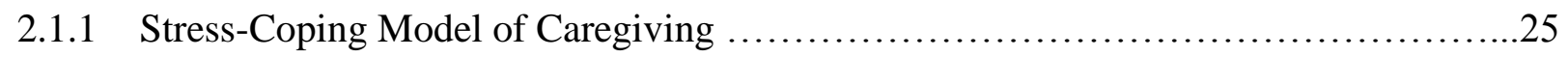

2.1.2 Circumplex Model of Family Functioning ....................................27

2.1.3 Strengths-Based Model of Family Resiliency ...............................28

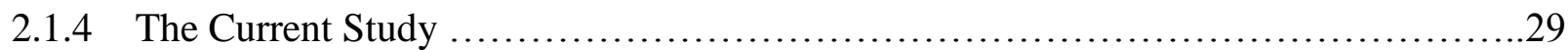

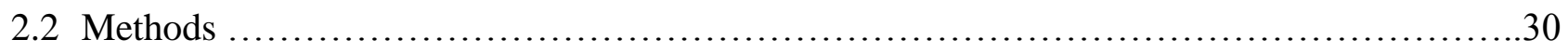

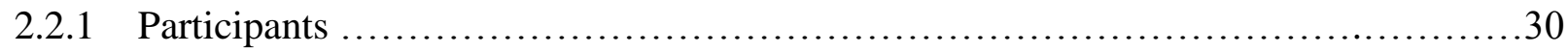

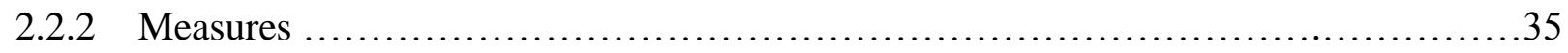

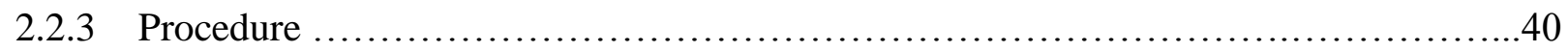

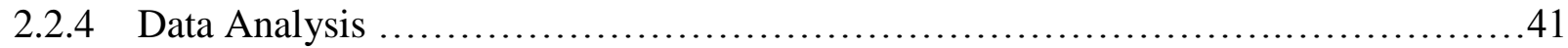

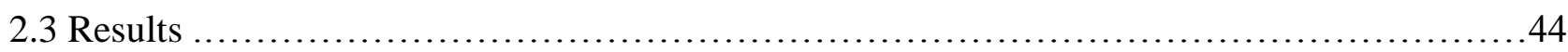

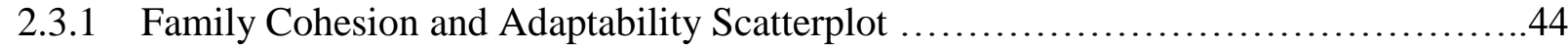

2.3.2 Chi-Square Analysis .................................................. 45

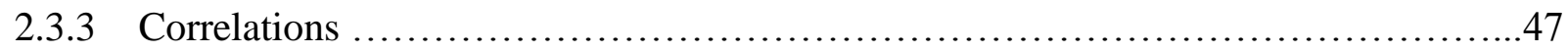

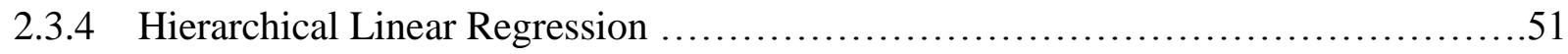

2.3.5 Stepwise Linear Regression . ...........................................52

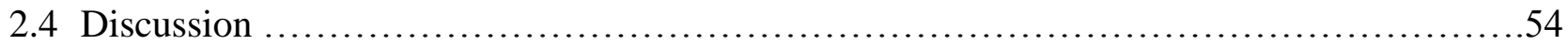

Chapter 3 Application of a Behavioural Paradigm to Understanding Family Functioning

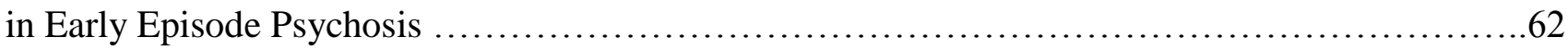

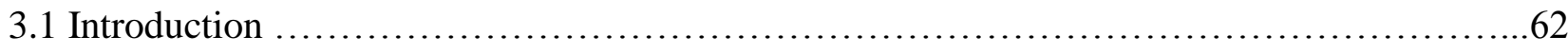

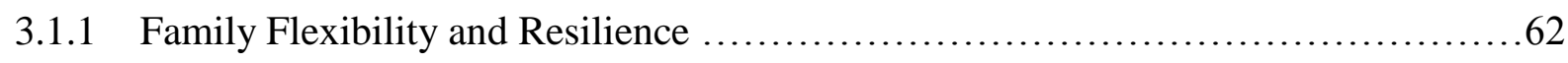

3.1.2 State Space Grids ..................................................65

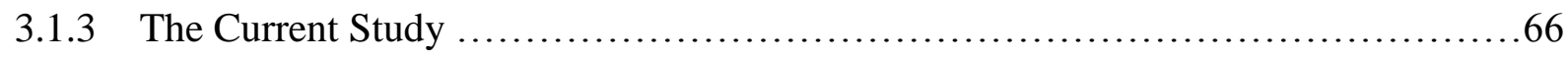

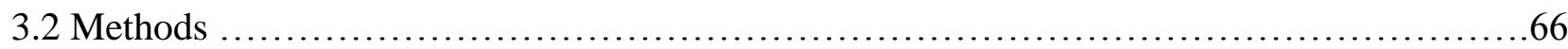




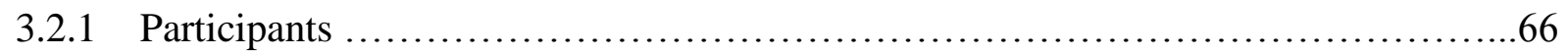

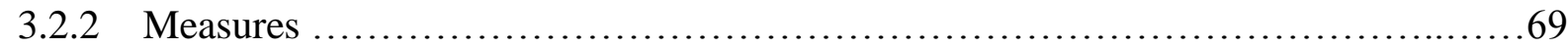

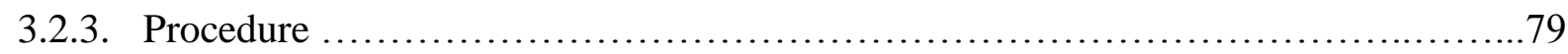

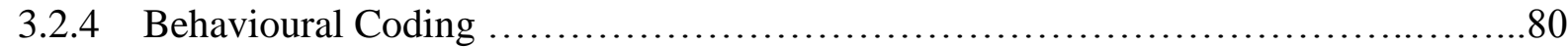

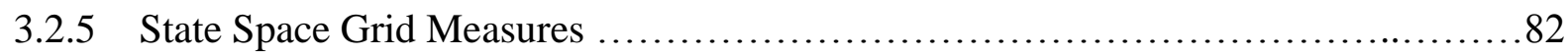

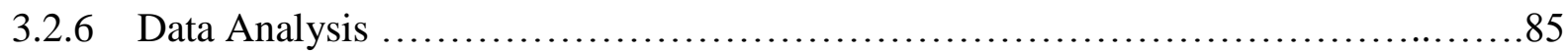

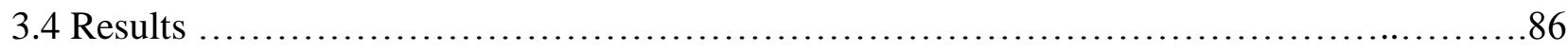

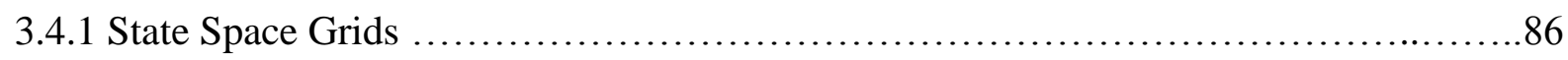

3.4.2 Summary State Space Grids ................................................. 92

3.4.3 Attractor Analyses ........................................................ 98

3.4.4 Repeated Measures Analysis of Variance (ANOVA) ................................ 101

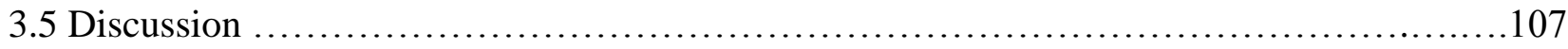

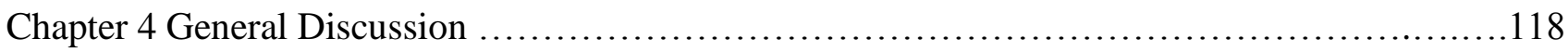

4.1 Broadening the Conceptualization of Family Functioning …............................118

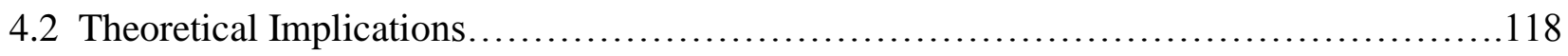

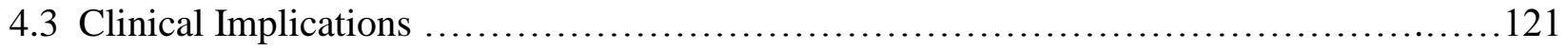

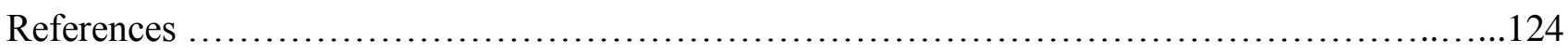

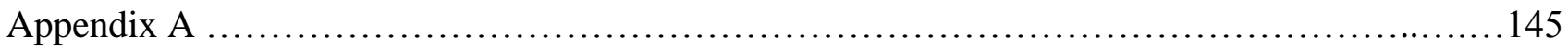

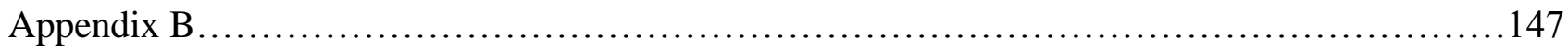




\section{List of Tables}

Table 2.1. Comparison of MTurk respondents and other online survey respondents.................32

Table 2.2. Means and standard deviations for participant characteristics and survey measures........36

Table 2.3. Observed and expected frequencies of families as a function of type of cohesion..........46

Table 2.4. Observed and expected frequencies of families as a function of type of adaptability........46

Table 2.5. Observed and expected frequencies of global family functioning $\ldots \ldots \ldots \ldots \ldots \ldots \ldots \ldots . . . . .47$

Table 2.6. Pearson's r correlation coefficients of the "distance from center" of the Circumplex model with youth clinical variables and caregiver variables.....................................48

Table 2.7. Pearson's r correlation coefficients among youth clinical variables, caregiver appraisals, coping, perceived helpfulness of supports, and caregiver and youth quality of life..................50

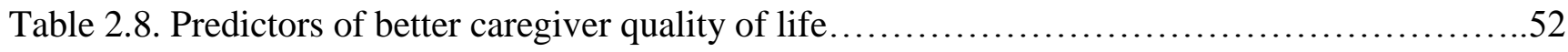

Table 3.1. Descriptive statistics for participant demographic data..............................69

Table 3.2. Frequency count for the FACES-III cohesion groups.............................76

Table 3.3. Frequency count for the FACES-III adaptability groups........................... 76

Table 3.4. Frequency count for the FACES-III general family functioning groups..................76

Table 3.5. Descriptive statistics for youth assessment measures..............................77

Table 3.6. Descriptive statistics for caregiver assessment measures $\ldots \ldots \ldots \ldots \ldots \ldots \ldots \ldots \ldots \ldots \ldots \ldots$

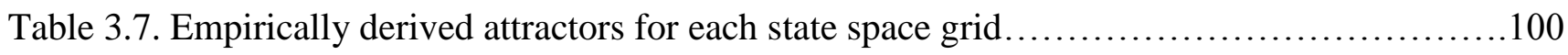




\section{List of Figures}

Figure 1.1 The Circumplex model of family functioning. The inner circle represents balanced levels of cohesion and flexibility.

Figure 1.2. A hypothetical state space grid for different emotional states of a parent and child during a brief interaction

Figure 1.3. State space grid examples of a flexible and rigid interaction between a caregiver and a youth.....

Figure 2.1. Consort chart. 34

Figure 2.2. Scatterplot of cohesion and adaptability scores.

Figure 2.3. Scatterplot of caregiver positive appraisals and family difficulties

Figure 3.1. State space grid for a positive family discussion between an individual with early episode psychosis and the primary caregiver.

Figure 3.2. State space grid for a family conflict discussion between an individual with early episode psychosis and the primary caregiver.

Figure 3.3. State space grid for a second positive family discussion between an individual with early episode psychosis and the primary caregiver.

Figure 3.4. State space grid for a psychosis-specific family conflict discussion between an individual with early episode psychosis and the primary caregiver.

Figure 3.5. State space grid for a third positive family discussion between an individual with early episode psychosis and the primary caregiver......................................................

Figure 3.6. Summary state space grids for the first positive family discussion by group...............94

Figure 3.7. Summary state space grids for the normative conflict discussion by group................95

Figure 3.8. Summary state space grids for the second positive discussion topic by group.............96

Figure 3.9. Summary state space grids for the psychosis-specific conflict discussion and follow-up positive discussion for the families with an individual with early psychosis.

Figure 3.10. Mean number of transitions (i.e., dynamic flexibility) for each discussion topic by group.

Figure 3.11. Dispersion scores (i.e., diversity of affective states) for each discussion topic by group. 
Figure 3.12. Duration of time in seconds per visit (i.e., rigidity) for each discussion topic

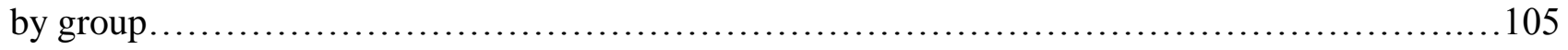

Figure 3.13. Entropy scores (i.e., unpredictability) for each discussion topic by group..............107 


\section{Chapter 1}

\section{General Introduction}

\subsection{Objectives}

Family functioning is a multi-faceted, complex, and ever-evolving phenomenon. Although family functioning plays an important role in one's recovery from psychosis, it remains relatively understudied in the early episode psychosis literature. To date, most of the research on family functioning in early episode psychosis focuses on unidimensional constructs (such as expressed emotion and family burden) that do not fully capture its complexity. My dissertation aimed to explore new avenues of measurement that would allow for a complex understanding of family dynamics in families with an individual with early episode psychosis. I employed two different methodologies to address existing limitations in this field of study. The first was the application of a family systems framework using the Circumplex model, wherein family functioning is assessed along dimensions of cohesion and flexibility. Although this model is well established in many other clinical fields, it has not been widely applied to the psychosis literature. This objective was also achieved with the use of state space grids to examine differences in emotional variability during in vivo family interactions. The use of parallel modes of measurement allows for a fuller and more comprehensive representation of family functioning. This project aimed to shed new light on the conceptualization and measurement of family functioning and resiliency in the early phases of psychosis. The overarching purpose of my dissertation was to better understand family systems and family interactions in early episode psychosis. My dissertation addressed the following 
research questions: (1) what factors contribute to adaptive family functioning during this critical period of illness?; (2) how do family interaction patterns manifest behaviourally in families with an individual with early episode psychosis?; and (3) how do these interaction patterns differ from healthy control families?

\subsection{An Overview of Psychosis}

Psychosis is a mental health condition that is characterized by a disconnection with reality resulting from the brain misperceiving stimuli in the external world. The most salient features of psychosis are hallucinations and delusions. Hallucinations are abnormal sensory and perceptual experiences that are experienced as reality by the individual, such as hearing voices that other people cannot hear. Although auditory hallucinations are most common, hallucinations can affect any of the human senses including visual, olfactory, and/or tactile functions (Tien, 1991). While hallucinations are defined as distorted sensory perceptions, delusions are defined as distorted thought processes. Delusions are beliefs that are firmly held despite contradictory evidence. Although many people hold beliefs that are based on incomplete information or incorrect assumptions, delusional beliefs are qualitatively distinct due to the absolute certainty with which those beliefs are held despite compelling contradictory evidence. Furthermore, delusional beliefs do not conform to common cultural beliefs that are normative within the individual's social and cultural environments. Both hallucinations and delusions are broadly classified as positive symptoms because they represent experiences that are in excess compared to healthy individuals. However, individuals with psychosis may also experience negative symptoms, which are termed negative because they represent deficits in behaviours that are typical of healthy individuals. Negative symptoms comprise of: (i) 
deficits in emotional expressivity that may include (but are not limited to) a reduction or flattening of facial expressivity, body gestures, and vocal qualities such as intonation, lack of emotional reciprocity, and reduced fluency of speech or content of speech, as well as (ii) deficits in motivation that may include social and emotional withdrawal, an inability to experience pleasure, or a reduction in personal interests, desires, and goals (Foussias \& Remington, 2010).

Symptoms of psychosis manifest in many different mental disorders. Psychosis is a necessary, but not sufficient, symptom in schizophrenia-spectrum disorders; however, it can also manifest in other disorders such as major depressive disorder, bipolar disorder, or dementia (DSM-5, 2013). Psychosis is also an exceptionally heterogeneous condition that is highly comorbid with other symptoms such as social anxiety, depression, substance abuse, obsessive compulsions, and trauma (Buckley, Miller, Lehrer, \& Castle, 2009; Strakowski, Keck, McElroy, Lonczak, \& West, 1995).

Analogous to other mental health conditions, psychosis occurs along a continuum and is experienced to varying degrees among all individuals. Prevalence estimates of positive symptoms (i.e., hallucinations and delusions) in healthy individuals range from 4\% (Eaton, Romanoski, Anthony, \& Nestadt, 1991) to 17.5\% (van Os, Hanssen, Bijl, \& Ravelli, 2000). A multitude of cross-sectional and longitudinal studies support the notion of psychosis as a dimensional phenomenon that is expressed along a continuum from normality to clinical disorder (e.g., Chapman et al., 1994; Hanssen et al., 2005; Peters, Joseph, \& Garety, 1999; Poulton et al., 2000; van Os, Hanssen, Bijl, \& Vollebergh, 2001). Provision of a clinical diagnosis is not based solely on the presence of psychosis, but on the duration of symptoms and the severity of functional impairment and/or 
subjective distress that results from the experience of psychosis (DSM-5, 2013;

Allardyce, Suppes, \& Van Os, 2007).

The trajectory of illness for psychosis has been well documented, although there are considerable individual differences in its onset, trajectory, and prognosis. In general, the onset of a first episode of psychosis occurs in late adolescence or early adulthood following a prodromal period wherein the individual experiences non-specific affective symptoms (e.g., low mood, distress), sub-threshold psychotic symptoms (e.g., hearing one's name being called, brief hallucinations that occur in the transition from wakefulness to sleep), and/or a gradual decline in social, academic, or occupational functioning (e.g., Jones et al., 1993; Yung et al., 1996). The prodromal period extends, on average, from 1 to 5 years until the onset of clear psychotic symptoms in a first episode of psychosis (Keith \& Matthews, 1991; Loebel et al., 1992; Beiser et al., 1993). The term "early episode psychosis" refers to the first five years of illness (Birchwood, Todd, \& Jackson, 1998). This phase marks a critical window in which intervention can occur. Early intervention services aim to detect and treat psychosis during the first few years of illness in order to improve long-term outcomes, reduce relapse, and prevent decline into more severe psychotic disorders such as schizophrenia (Bertolote \& McGorry, 2005; McGorry, Killackey, \& Yung, 2008).

\subsection{Early Intervention for Psychosis}

The early intervention movement was pioneered almost 20 years ago in Melbourne, Australia (McGorry et al., 1996). Today, early intervention for psychosis has become a mainstream part of psychiatric services worldwide and services are available in hundreds of locations across the UK, Europe, Asia, and North America. The emergence 
of early intervention for psychosis required a widespread paradigm shift in the way that researchers and clinicians conceptualized psychosis. Early definitions of schizophrenia in the $19^{\text {th }}$ century suggested that schizophrenia was a degenerative disorder caused by a gradual deterioration of the brain (Kraeplin, 1919). This early conceptualization shaped a pessimistic view about the prognosis and long-term outcomes for individuals with a psychotic disorder. Today, there is an abundance of evidence suggesting that psychotic disorders are not inherently degenerative and that there is variability in the course of illness. Although some individuals with psychosis experience a gradual worsening of symptoms, some will show a complete remission of symptoms naturally, while others will experience an episodic course of illness with periods of time where functioning is restored to premorbid levels (Bertelsen et al., 2009; Harrison et al., 2001; Huber, Gross, \& Schuttler, 1975). Most importantly, there is empirical evidence for the benefits of pharmacological and psychological intervention wherein individuals experience improvements in symptoms and functioning in response to adequate treatment (e.g., Baldessarini, Cohen, \& Teicher, 1988; Bird et al., 2010).

The premise of early intervention is based on an extensive body of literature that suggests that the duration of untreated psychosis (DUP) is a critical indicator of illness prognosis. It is well established that a longer DUP is associated with poorer outcomes (Marshall et al., 2005). Intervention during these early stages of psychotic illness aims to prevent further decline and promote better long-term functional outcomes (Bertolote \& McGorry, 2005; Killackey \& Yung, 2007; McGorry et al., 2008) and has been shown to be more cost effective than standard care (Mihalopoulos, Harris, Henry, Harrigan, \& McGorry, 2009). Early interventions services consist of a combination of 
pharmacological and psychosocial interventions (Addington, 2007). However, the exact permutation of services offered varies considerably from site to site. Highly funded clinics that are affiliated with an academic institution often offer the greatest variety of treatment services. Treatment can include some combination of antipsychotic medication, cognitive behavioural therapy, cognitive remediation therapy, family therapy, and supported employment (Addington, 2007; Rinaldi et al., 2004; Wykes et al., 2007). However, the reality of early intervention is often different from what is reported in these large-scale clinical academic studies, as not all clinics have the staffing or resources to provide the gold-standard complement of services. Some services can only afford to offer basic case management and pharmacological follow-up, without many options for individual therapy, group therapy, or skills-based interventions. There remains to this day a large gap between research on early intervention and its application in everyday clinical practice. Given this gap, the field has started to examine the mechanisms of early intervention in order to develop a better understanding of the key components that are most important to include in early intervention services.

One of the critical components that has been identified is family support and psychoeducation (Addington, McKenzie, Norman, Wang, \& Bond, 2013). Several studies have reported on the need to provide support to family members and the importance of including families in treatment planning (e.g., Addington, Collins, McCleery, \& Addington, 2005; Bertolote \& McGorry, 2005; Birchwood et al., 1998; International Early Psychosis Association Writing Group, 2005). Many guidelines for early intervention services include a section on family education and support. For example, the Early Psychosis Intervention Standards for Ontario encourage a family-centered approach 
for both assessment and treatment, including education about the family's roles and responsibilities (Ministry of Health and Long Term Care, 2011). The evidence in support of family intervention for psychosis is such that it is now considered to be an evidencebased treatment (Lehman et al., 2004; Addington et al., 2005).

Given that the age of illness onset for psychosis falls around adolescence and early adulthood, many individuals are at an age where they still live at home, or are in close contact with family, when symptoms emerge. As such, families are often included as active participants in the recovery process. Although the importance of including family members and providing family support is well established, very little is known about the factors that initiate and maintain adaptive family functioning in this population. This is especially important given the unique stressors that early episode psychosis places on the family unit. Published research in this area to date has largely focused on onedimensional constructs such as expressed emotion or caregiver burden, which don't account for family functioning as a multi-faceted phenomenon. As such, an overview of studies that have examined family functioning in early episode psychosis, their limitations, and new directions for research are described below.

\subsection{Family Functioning in Early Episode Psychosis}

Understanding family functioning is a widespread research pursuit, and the research literature in psychology and sociology is overflowing with family studies in both clinical and healthy populations. However, the theoretical underpinnings, research methodologies, and measurement tools vary considerably not only from field to field but also study to study. In this paper, I will focus on the literature most relevant to my population of interest - early episode psychosis. To date, the field of family functioning 
in early episode psychosis has focused almost exclusively on two constructs: expressed emotion and family burden (Koutra et al., 2014a).

\subsubsection{Expressed Emotion}

Expressed emotion is a long-standing concept that refers to the family's negative emotional environment and is conceptualized as three different components: (i) critical comments, (ii) hostility, and (iii) emotional over-involvement such as over-protectiveness towards the individual with psychosis (Kuipers, 1992; Scazufca \& Kuipers, 1996; Vaughn, 1989). Expressed emotion is typically measured by clinician-ratings of criticism, hostility, and emotional over-involvement based on interviews with family members. A recent review by Koutra and colleagues in 2014 concluded that similar to families of an individual with chronic schizophrenia, families with an individual with early episode psychosis exhibit similar prevalence rates of expressed emotion. Estimates of early psychosis families that display high levels of expressed emotion range from $35 \%$ (Meneghelli et al., 2011) to 74\% (McNab et al., 2007).

Illness characteristics, such as symptoms and psychosocial functioning, appear to have a minimal impact on caregivers' expressed emotion (Bachmann et al., 2002; Heikkila et al., 2002; Meneghelli et al., 2011; Möller-Leimkuhler, 2005; Raune, Kuipers, Bebbington, 2004); rather high expressed emotion is associated with relatives' internal appraisals about the illness (González-Blanch et al., 2010; Patterson, Birchwood, \& Cochrane, 2005; Raune et al., 2004). Furthermore, although years of research have demonstrated that high expressed emotion predicts relapse in schizophrenia (Butzlaff \& Hooley, 1998; Cechnicki, Bielanskab, Hanuszkiewiczb, \& Darenb, 2013; Hooley, 2007), this relationship is unclear in early episode psychosis, with some studies finding an 
association between high expressed emotion and relapse (Barrelet, Ferrero, Szigethy, Giddey, \& Pellizzer, 1990; Huguelet, Favre, Binyet, González, \& Zabala, 1995; Lee, Barrowclough, \& Lobban, 2013), and others finding no significant association between these two variables (Sterling et al., 1991; Sterling et al., 1993). Some researchers suggest that this may be due in part to differing attitudes towards the individual with psychosis, as relatives of an individual with early episode psychosis may not be biased by the experience of repeated relapses (Bachmann et al., 2002).

\subsubsection{Family and Caregiver Burden}

The construct of family and caregiver burden has received substantial attention in the research literature. In the past, researchers would distinguish between objective and subjective burden, where objective burden indicated effects on the household (e.g., financial loss, disruption of family routines, and loss of work productivity), whereas subjective burden indicated the extent to which caregivers perceived the burden of care (e.g., difficulty coping with the illness and the emotional climate at home). However, recent studies have found that caregivers can experience objective burden and subjective satisfaction with the caretaking role, and as such the term burden has largely been replaced with the term "caregiver experience" (Treasure et al., 2001; Martens \& Addington, 2001).

Caregiver experiences can encompass distress, stigma, worry, shame, as well as positive elements such as reward or fulfillment (Szmukler et al., 1996). Approximately one third of caregivers have symptoms of depression or anxiety related to their caretaking role (Goldberg \& Hillier, 1979; MacCarthy, Lesage, Brewin, 1989), although some studies report as low as $12 \%$ of caregivers with psychiatric symptoms of their own 
(Tennakoon et al., 2000). Predictors of negative caregiver experiences include a greater number of disruptive patient behaviours, increased patient disability, and greater severity of the patient's psychotic symptoms (Ohaeri, 2003), as well as greater severity of depressive symptoms (Tucker, Barker, \& Gregoire, 1998). However, these studies do not account for the caregiver's own internal psychological resources and coping strategies. When caregiver resources are taken into account, caregiver appraisals or coping strategies are more closely related to the caregiver's wellbeing than the severity of the family member's symptoms (Addington, Codham, Jones, Ko, \& Addington, 2003). Caregiver stress is independent of objective stressors such as the severity of the patients' illness, type of symptoms, and level of functioning; rather negative caregiver experiences are associated with the psychological resources and the disposition of the relatives themselves (Möller-Leimkühler, 2005).

Over time, research on caregiver burden has also led to the conceptualization of caregiving within a 'stress-coping' framework (Lazarus \& Folkman, 1984; Szmukler et al., 1996), where the caregiving experience is conceptualized as the appraisal of its demands. This can refer to both positive and negative appraisals of caregiving. In general, the 'stress-coping' model of caregiving suggests that caregivers experience stressors such as the needs of their family member and illness severity, but that there are other factors such as social support that mediate the effect of those stressors on the caregivers' appraisals. The model suggests that the interaction between the caregivers' appraisals and coping strategies predicts their psychological wellbeing (Joyce et al., 2003; Joyce, Leese, \& Szmukler, 2000; Szmukler et al., 1996). 


\subsubsection{Limitations and New Directions}

The literature on family functioning in early episode psychosis to date has focused primarily on expressed emotion and family burden; however, these constructs are inherently deficit-focused and do not adequately address the multifaceted nature of family functioning and the complexity of a dynamic and ever-changing family system. The field is undergoing a paradigm shift where it is moving away from a deficits-based approach and towards a strengths-based approach that focuses on family resilience, adaptive family coping, measuring family functioning along dimensions of cohesion and flexibility, and understanding how families bounce back from stress. A recent review of family functioning in early episode psychosis made a call to arms for the need to examine family functioning based on dimensional constructs of family cohesion and flexibility, rather than expressed emotion and burden (Koutra et al., 2014a). My dissertation aims to answer that call by exploring new avenues of measurement that allow for a complex understanding of dynamics in families with an individual with early episode psychosis. One way in which this goal was achieved was by examining family functioning within the family systems theoretical framework of the Circumplex model (Olson, 2000), wherein family functioning is assessed along two dimensions: cohesion and flexibility. Although this model is well established in many other clinical fields, it has not been widely applied to the psychosis literature. The other method with which this goal was achieved was through the application of dynamic systems theory and state space grids.

\subsection{The Circumplex Model of Family Functioning}

In the Circumplex model, family functioning is conceptualized as an interaction of family cohesion and family adaptability (Minuchin, 1974; Olson et al., 1979; 2000). 
Family cohesion refers to the emotional bond that family members develop toward one another, whereas family adaptability refers to the ability of the family system to alter its power structure, roles, and relationship rules in response to situational and developmental stress. The Circumplex model proposes four levels of cohesion, which include (1) disengaged, (2) separated, (3) connected, and (4) enmeshed, as well as four levels of adaptability include (1) rigid, (2) structured, (3) flexible, and (4) chaotic (please refer to Figure 1.1). Family cohesion and adaptability are curvilinear variables, wherein both extremely low and extremely high levels of cohesion and adaptability are maladaptive and associated with poor family outcomes. Healthy families have been found to display balanced levels of these traits (Henggeler, Burr-Harris, Borduin, \& McCallum, 1991; Rodick, Henggeler, \& Hanson, 1986). 


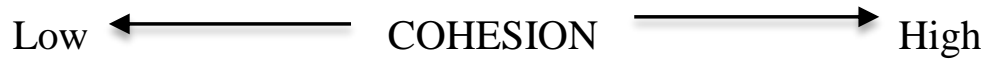

\section{Disengaged Separated Connected Enmeshed}

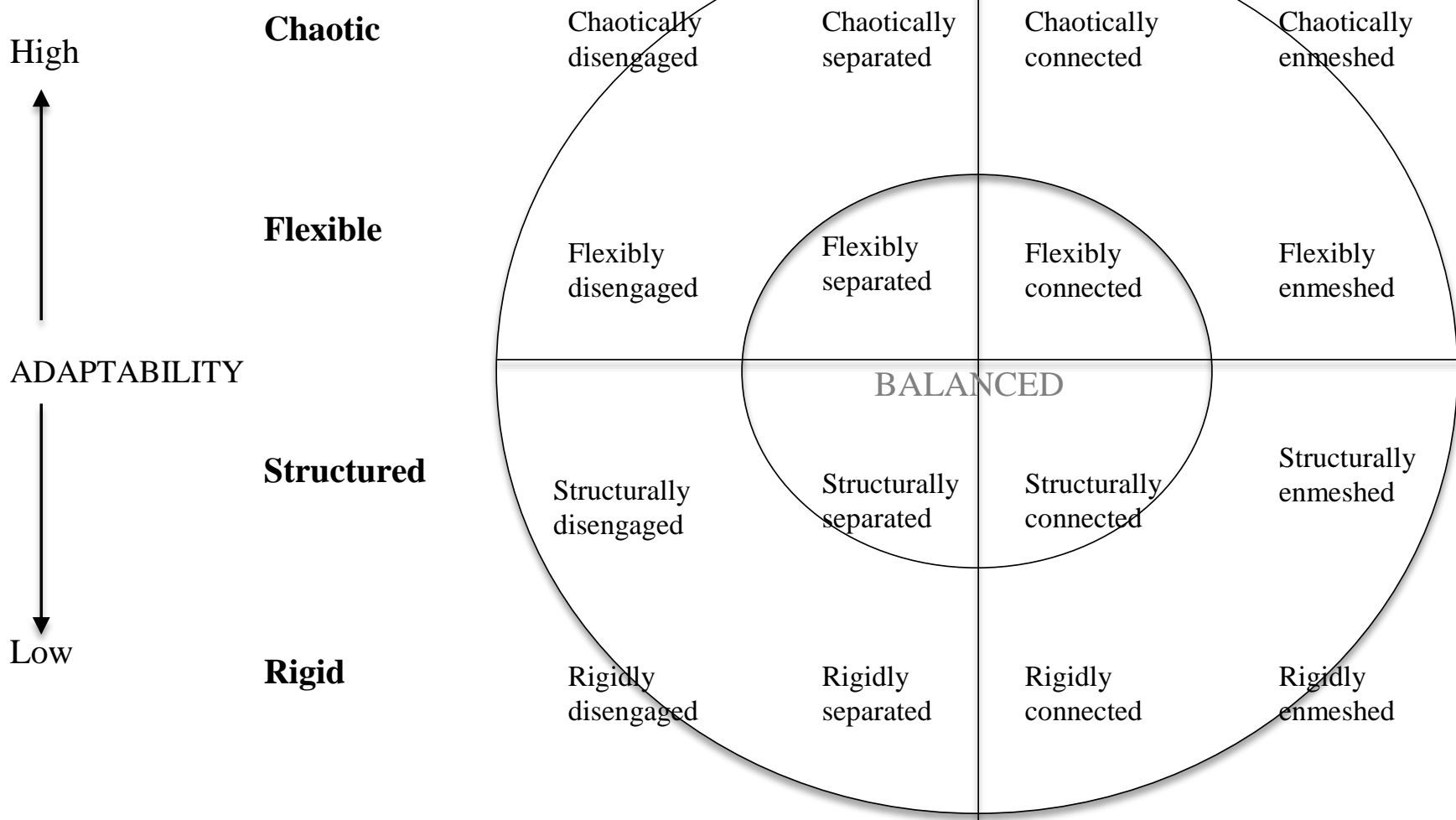

Figure 1.1. The Circumplex model of family functioning. The inner circle represents balanced levels of cohesion and flexibility.

\subsection{Dynamic Systems and State Space Grids}

\subsubsection{Dynamic Systems Theory}

Dynamic systems theory is a scientific approach that aims to understand the structure of relationships within complex behavioural systems and how these systems stabilize over time (Prigogine \& Stengers, 1984). Dynamic systems theory postulates that all open systems have bi-directional relationships among system elements that occur within and across micro, meso, and macro levels (Hollenstein, 2013; Hollenstein, 
Lichtwarck-Aschoff, \& Potworowski, 2013). The micro level is the bottom level that is compromised of the most simple system components (e.g., moment-to-moment processes), the meso level is the intermediate level that is made up of interactions at the micro level (e.g., behaviour that occurs over minutes to hours), and the macro level is the top (and most complex) level that is made up of interrelations among the systems at the meso level as they unfold over time (e.g., occurring over months or years; Hollenstein, 2013). This process of interactions across multiple levels of a system is called selforganization. Self-organization can be both bottom-up (i.e., where low level elements cascade towards the superordinate structure of the system) and top-down (i.e., where higher order interactions influence the rudimentary components of the system). These principles can be applied to numerous systems, physical and behavioural, including celestial systems, thermodynamic systems, and of relevance to this project - family systems (Hollenstein, 2013). Before reviewing how principles of dynamic systems theory apply to family research and existing studies in this area to date, it is first important to provide an overview of key concepts such as attractors and state space grids.

\subsubsection{Attractors}

Self-organizing systems have many possible states but often have only a few specific states that are stable and occur regularly. These recurring states are called "attractors", and are aptly named as they attract or pull a system towards that particular state (e.g., Granic \& Lamey, 2002; Johnson \& Nowak, 2002). For example, depression has been described as an attractor as it is an emotional state that has a high probability of re-occurrence and from which it is difficult or effortful to emerge once pulled into that state (Johnson \& Nowak, 2002). When thinking about family systems, attractors are 
conceptualized as recurring patterns of interactions between family members that develop and stabilize over time (e.g., Fogel, 1993; Fogel \& Thelen, 1987, Hollenstein, Granic, Stollmiller, \& Syder, 2004).

\subsubsection{State Space Grids}

State space grids provide a visual model for the range of behavioural possibilities for any given system, and allow for the examination of variability and stability of different interaction patterns as they unfold (Lewis, Lamey, \& Douglas, 1999). State space grids are grids depicted in two dimensions along an $x$-axis and $y$-axis. Each axis represents a variable and each cell in the grid represents a mutually exclusive state for that variable. A hypothetical state space grid for a parent-child interaction is presented in Figure 1.2. In this example, the parent is represented along the $x$-axis and the child is represented along the $y$-axis. The cells in this grid represent different emotional states of the parent and child, such that each cell indicates the intersection of the parent and child's behaviour. In this example, the states are labeled: positive affect, interest, neutral, withdrawal, and hostility. Each plot point (or node) on the state space grid represents an event and the size of that node indicates how long the dyad was in that state. The hollow node represents the very first state of the dyad and each node's position within the cell is randomly generated. The lines represent the dyad's transition to different states as they visit different cells on the grid. Figure 1.2 is a hypothetical state space grid for a parent and child discussion. Both the parent and child started the conversation in neutral. The parent then displayed interest and curiosity about the child while the child remained in neutral. Then, as the parent remained in interest, the child displayed positive affect, 
which was reciprocated by the parent, and the dyad ends in their conversation in mutual positivity.

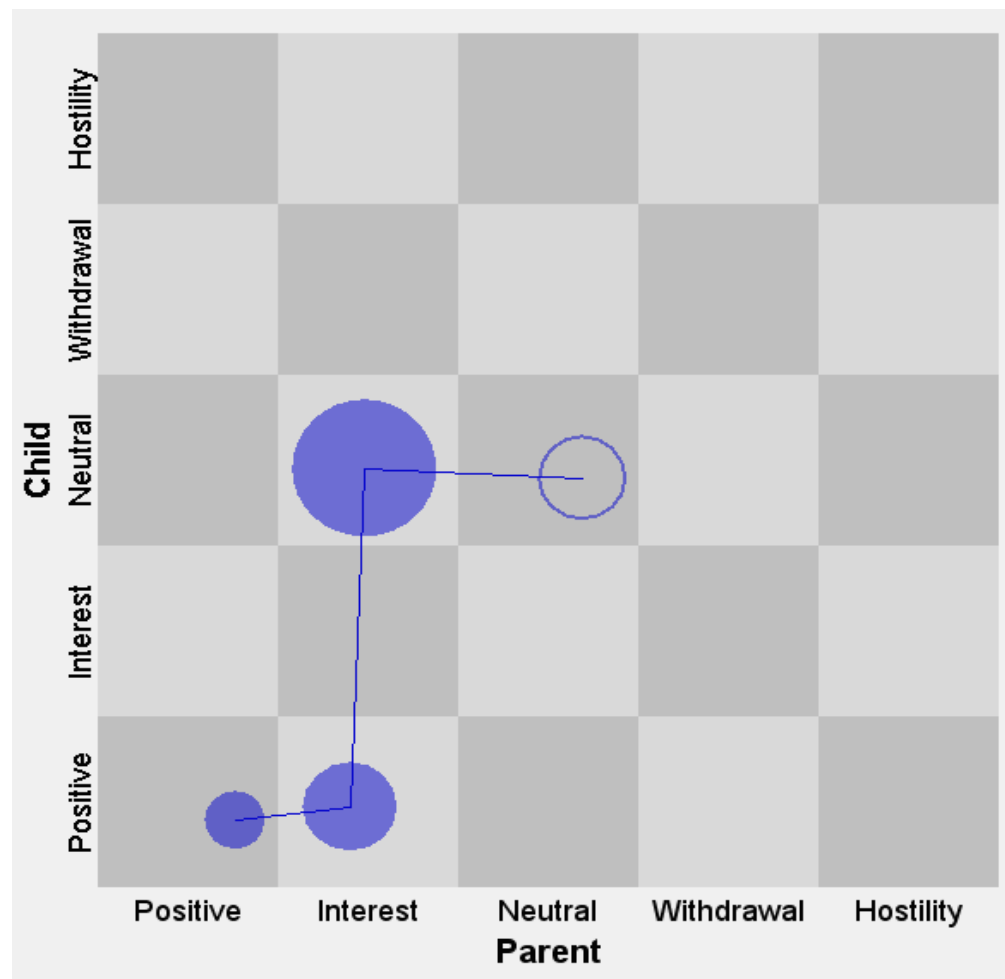

Figure 1.2. A hypothetical state space grid for different emotional states of a parent and child during a brief interaction.

In addition to the visual illustration of dyadic interactions in real time, state space grids also provide quantitative measures of variability. Within the field of dyadic family interactions, variability, sometimes referred to as flexibility, represents the ability to adapt to changes in the environment (Hollenstein et al., 2013). Several research studies (described below) have indicated that a lack of variability in a dyadic family interaction is related to psychopathology and clinical outcomes. Four measures of variability can be obtained from state space grids: (1) dynamic flexibility, which is the number of transitions between different states on the grid represented by the trajectory line on the 
grid, (2) diversity or range of affective and behavioural states, (3) rigidity, or the tendency to get stuck in a particular state as measured by the duration of time spent in a particular state, and (4) unpredictability in the sequence of events during an interaction measured as entropy. Each of these four measures will be described in detail in Chapter 3. In general, variability indicates movement across the grid, where participants frequently enter and exit different states, whereas its counterpart, rigidity, is a restricted pattern of fixed behaviours. Flexibility and rigidity can be viewed as opposites ends of a single dimension (Hollenstein et al., 2013). Please refer to Figure 1.3 for an example of both a flexible and rigid caregiver-youth interaction. In the flexible example (on the left) the two family members frequently shift between positive, interest, and neutral states. In the rigid example (on the right) the two family members remain in only a few states for longer periods of time. 
Flexible

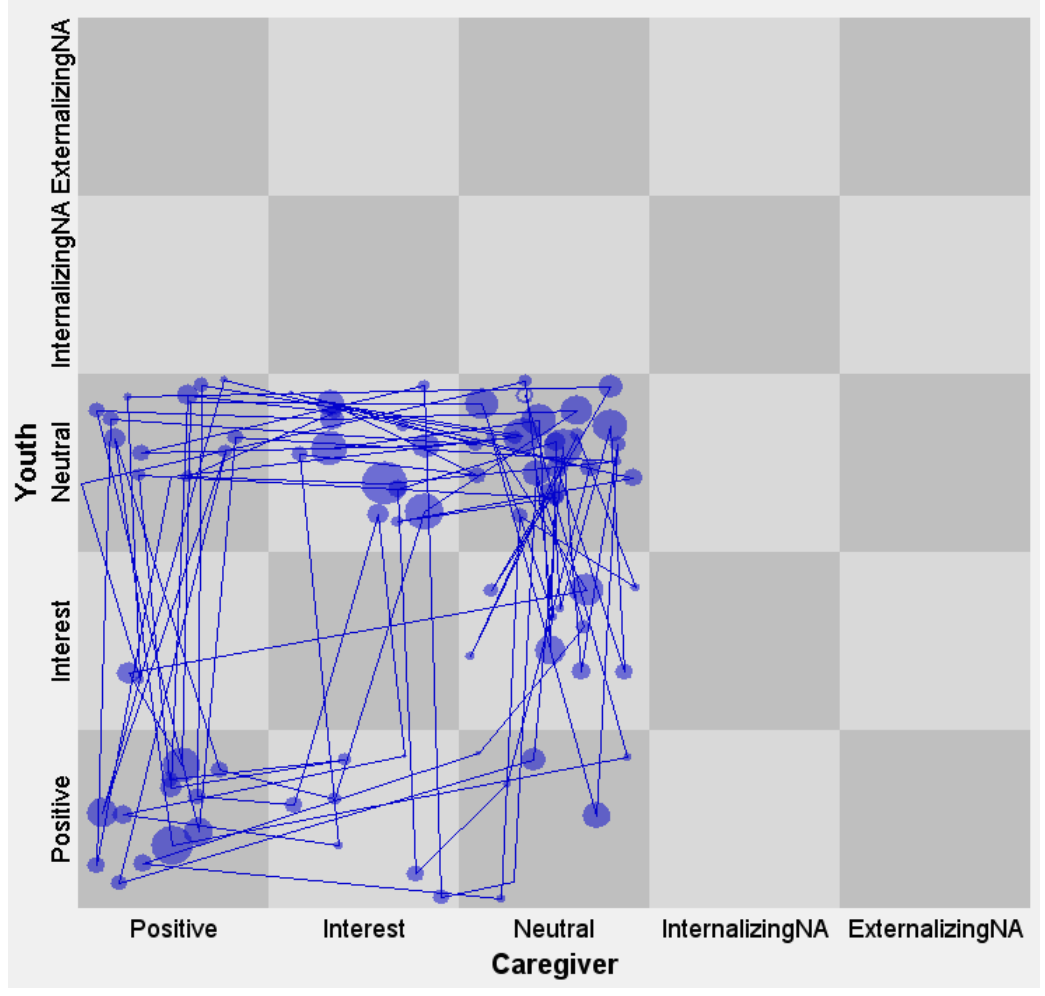

Rigid

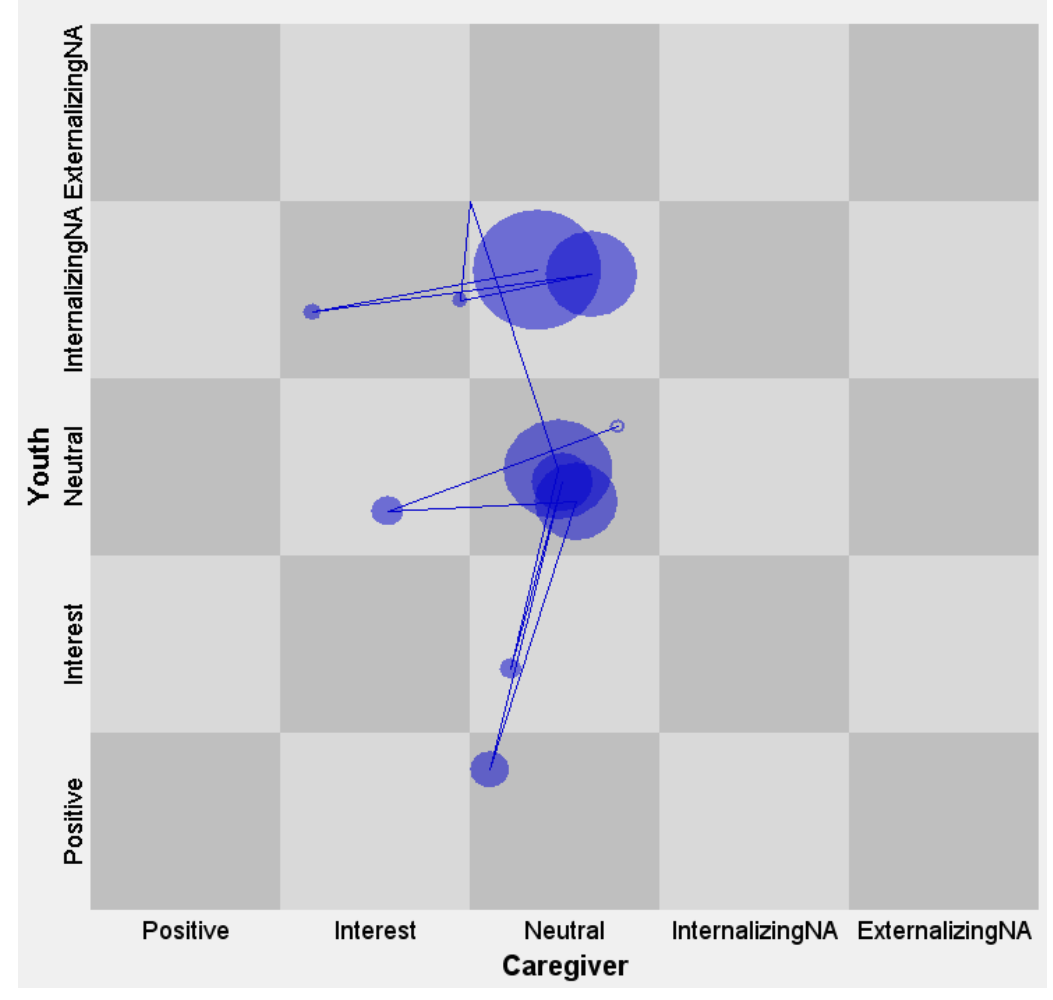

Figure 1.3. State space grid examples of a flexible and rigid interaction between a caregiver and a youth. 


\subsubsection{An Overview of Key State Space Grid Studies}

An overview of state space grid studies to date demonstrates its versatility in the behavioural sciences. The initial state space grid study, in which the method was first introduced, was conducted by Lewis, Lamely, and Douglas in 1999 where they used state space grids to examine the socioemotional development of infants. Infants were given frustrating toys to play with when in close proximity to their mother, and the state space grid included distress intensity on one axis and angle of gaze to the mother on the other axis. The authors noted individual and developmental changes in behaviour that would not have been apparent with traditional research methods. Since this seminal paper was published, state space grids have been expanded to examine parent-child interactions, primarily with children with externalizing and internalizing behaviour problems. Several influential articles have been published in this area and form the theoretical basis for the study presented in Chapter 3.

In 2002, Granic and Lamey first used state space grids to examine parent-child interactions in children with externalizing behaviour problems. The researchers examined two groups, pure externalizing children and mixed externalizing and internalizing children. Each dyad had six minutes to discuss a family conflict. These discussions were video recorded and coded for the parent and child's behaviour and affect. The researchers also introduced a perturbation in their design - where the research assistant knocked on the door with two minutes left and asked the dyad to "wrap up on a good note". Perturbations increase the pressure on a family dyad and cause instability within the system, revealing interaction patterns that would otherwise be unknown. Interestingly, the authors found that although the two groups looked quite similar in their interaction 
patterns prior to the perturbation, after the perturbation only those with mixed behaviour problems entered a state of mutual hostility. This was the first study to demonstrate how state space grids could reveal interesting patterns of interactions that would be missed by the use of static self-report or clinician-rated measures.

Variability measures derived from state space grids have also been examined in families. Overall, many studies have revealed that flexible family interactions are adaptive whereas rigid family interactions are maladaptive. For example, one study found associations between rigidity in parent-child interactions and externalizing behaviour problems, increases in externalizing problems over time, and chronic levels of internalizing problems in high risk kindergarten children (Hollenstein, 2004). Flexibility in parent-child interactions is correlated with behavioural ratings of emotion regulation (Lunkenheimer, Hollenstein, Wang, \& Shields, 2012) and with cortical mediators of emotion regulation in children with aggression problems (Lewis et al., 2006). Flexibility in dyadic interactions is also relevant to adolescent development. For example, dyadic flexibility between mothers and adolescents played an important role in those adolescents' ability to adaptively manage conflict (van der Giessen, Branje, Keijsers, Van Lier, Koot, \& Meeus, 2014) and less flexibility has been found to predict increases in mothers' internalizing behaviours and adolescent symptoms of depression and anxiety five years later (Van der Giessen, Hollenstein, Hale, Koot, Meeus, \& Branje, 2015). Given the important role of family flexibility in clinical outcomes, emotional regulation, and development, flexibility has become a primary variable of interest in this field of research. 
A popular study design in the application of state space grids involves an experimental manipulation where families are asked to engage in a variety of positive and negative family discussions, following an ABA design (positive - negative - positive). Families are provided with a standard positive discussion topic (e.g., plan a fun family vacation) or a conflict discussion topic that is personally meaningful to them (e.g., fighting with siblings), and allowed several minutes to talk and resolve the issue. These family discussions are video recorded and coded for different emotional expressions. Researchers have found that flexibility in emotional expression is restricted during conflict, but not positive discussions, indicating that in general, negative emotion may restrict interpersonal flexibility (Hollenstein \& Lewis, 2006). An important article by Granic and colleagues in 2007 applied the same ABA design of positive and negative family discussion topics and examined state space grids as an outcome measure for a family intervention directed at parents of children with externalizing behaviour problems. The authors found that improvements in children's externalizing behaviour following 12weeks of cognitive-behavioural therapy was associated with greater flexibility during the conflict discussion but that there was no change in flexibility in non-improvers, marking flexibility as a successful treatment outcome measure.

\subsection{Towards a Better Understanding of Family Functioning in Early Psychosis}

Flexibility is an important component of family functioning; however, the definition of flexibility can vary considerably. In the Circumplex model, flexibility is defined as the ability of a family system to change its power structure, roles, and relationship rules in response to situational and developmental stress (Olson, Sprenkle, \& Russel, 1979). In the dynamic systems literature, however, flexibility refers to emotional 
variability and the ability to transition in and out of affective states (Hollenstein et al., 2013). This type of emotional flexibility can either be moment-to-moment changes in emotion that occur within the same context, or changes in emotion that are in response to alterations in environmental or contextual demands (i.e., shifting emotion because the situation has changed; Hollenstein et al., 2013). Although the Circumplex and dynamic systems definitions of flexibility are different (one being changes in family structure and the other being changes in and out of emotional states), these constructs do conceptually overlap to some degree. Both underscore the importance of being able to adapt to changing environmental demands. As previously described, flexibility is associated with better socioemotional and family functioning across both theories. These two definitions of flexibility provide a different, yet somewhat overlapping, framework from which family systems can be conceptualized and understood, and both of these concepts will be explored in this paper.

The overarching aim of this dissertation is to build upon previous work on family functioning in early episode psychosis by applying multi-faceted, dimensional, and dynamic tools of measurement to better understand family cohesion and flexibility in this population. Chapter 2 presents an online caregiver survey that examined relationships among various caregiver and family system variables in an effort to replicate the stresscoping model of caregiver wellbeing and develop a foundation for a new model of family cohesion and flexibility. Chapter 3 presents the novel application of state space grids to examine the behavioural manifestation of family interaction patterns in real time and determine differences in family interaction patterns between families with early episode psychosis and healthy control families. Finally, Chapter 4 provides a general discussion 
about the clinical implications of this research and what it means for clinicians and family members who are supporting individuals with psychosis during their recovery. 


\section{Chapter 2}

\section{Caregiver Appraisals and Family Functioning in Early Episode Psychosis}

\subsection{Introduction}

Family involvement is a cornerstone of early intervention services for psychosis. Family members are often included in treatment planning and are active participants in the recovery process (McCann, Lubman, \& Clark, 2011). In fact, the mandate for many early psychosis intervention services across Canada is to provide support and promote recovery, not only for the individual with psychosis, but for the whole family.

Family involvement is particularly crucial in early episode psychosis in large part because of the typical age of illness onset. The onset of a first episode of psychosis commonly occurs in late adolescence or early adulthood following a prodromal period wherein the individual experiences non-specific affective symptoms, sub-threshold psychotic symptoms, and a gradual decline in functioning (e.g., Jones et al., 1993; Yung et al., 1996). As such, many individuals with psychosis are still in contact with or living with family during the early phases of illness (Addington, 2004). The early stages of psychosis are often a chaotic, frightening, and stressful period for individuals and their

families, who struggle to understand the symptoms of the illness and access appropriate mental health services (Addington et al., 2003; Barker, Lavender, \& Morant, 2001; Gerson et al., 2009; Jones, 2009; Knock et al., 2011; McCann et al., 2011).

Importantly, the early phases of psychosis mark a critical period in which early intervention can occur. Early intervention has become a rapidly developing field that has received international attention and has now become a part of mainstream psychiatric 
services. Early intervention for psychosis has been shown to reduce the reoccurrence of psychotic symptoms following a first episode of psychosis (Robinson et al., 1999; 2004), lead to greater functional recovery, lower the risk of progression to more severe forms of psychotic disorder, and reduce mortality related to comorbidities such as substance use, smoking, social anxiety, depression, and suicide (see McGorry \& Yung, 2003). Furthermore, early intervention has been shown to be more cost effective than standard psychiatric care (McCrone et al., 2013). Family education and support are crucial parts of any early intervention program for psychosis (Addington et al., 2013) and the evidence in support of family intervention for psychosis is such that it is now considered to be an evidence-based treatment (Lehman et al., 2004; Addington et al., 2005). Components of evidence-based family intervention include illness education, crisis intervention, emotional support, and coping skills training (Lehman et al., 2004). Although the importance of family support and intervention for early psychosis is well known, very little is known about the factors that initiate and maintain adaptive family functioning in this population.

The theoretical framework for the current study on family functioning in early episode psychosis is based on three existing theoretical models: the Stress-Coping Model of Caregiving, the Circumplex Model of Family Functioning, and the Strengths-Based Model of Family Resiliency.

\subsubsection{Stress-Coping Model of Caregiving}

Caregiver burden and caregiver wellbeing are important considerations in family functioning. Approximately one-third of caregivers of an individual with psychosis have symptoms of depression or anxiety related to their caretaking role (Goldberg \& Hillier, 
1979; MacCarthy et al., 1989), although some studies report as low as $12 \%$ of caregivers with psychiatric symptoms of their own (Tennakoon et al., 2000). However, caregiving is undoubtedly complex, and encompasses both positive and negative experiences. For example, caregivers' experiences can encompass negative experiences such as distress, stigma, worry, shame, as well as positive elements such as reward and fulfillment (Szmukler et al., 1996). Negative caregiver experiences are associated with a greater number of disruptive behaviours from the family member with psychosis, increased disability of their family member with psychosis, and greater severity of their family member's psychotic symptoms (Ohaeri, 2003) and depressive symptoms (Tucker et al., 1998), as well as reduced working memory and executive functioning (Hjärthag et al., 2010). Interestingly, when caregivers' coping strategies and internal psychological resources are taken into account it is not the severity of their family member's symptoms, but the caregivers' internal appraisals of the illness that are most closely related to caregiver wellbeing (Addington et al., 2003). This research suggests that caregivers' own psychological resources are particularly important in being able to manage the stress associated with having a family member with psychosis.

Caregiving is often conceptualized within a 'stress-coping' framework (Lazarus \& Folkman, 1984; Szmukler et al., 1996), where the caregiving experience is conceptualized as the appraisal of its demands. This can refer to both positive and negative appraisals of caregiving. The 'stress-coping' model of caregiving suggests that caregivers experience external stressors (such as the needs of the family member and illness severity), but that caregiver outcomes (such as their own wellbeing) are not directly associated with the stressors themselves, but to the cognitive appraisals of the 
illness and its consequences, and the coping strategies they employ. The model suggests that both the caregivers' appraisals and coping strategies predict their psychological wellbeing. The stress-coping model has received support over the years with evidence for a relationship between caregiver appraisals and their psychological wellbeing (Joyce et al., 2000; Joyce et al., 2003; Szmukler et al., 1996). However, the stress-coping model of caregiver wellbeing has yet to be applied to our understanding of family wellbeing. Understanding family wellbeing requires treating the family as a dynamic and evolving system, at a level above the individual.

\subsubsection{Circumplex Model of Family Functioning}

When examining family systems in the general family literature, family functioning has been conceptualized as an interaction of family cohesion and family adaptability (Minuchin, 1974; Olson et al., 1979; 2000). In this model, called the Circumplex model of family functioning, family cohesion is defined as the emotional bond that family members develop toward one another, whereas family adaptability is defined as the ability of the family system to alter its power structure, roles, and relationship rules in response to situational and developmental stress. In the Circumplex model of family cohesion and adaptability, cohesion and adaptability are curvilinear variables, wherein both extremely low and extremely high levels of cohesion and adaptability can be maladaptive and are associated with poor family outcomes, whereas healthy families display balanced levels of these traits (Henggeler, Burr-Harris, Borduin, \& McCallum, 1991; Rodick, Henggeler, \& Hanson, 1986). This model was initially developed by Olson and colleagues in 1979, and has since been used in numerous studies, including studies on families with a depressed adolescent (Cumsille \& Epstein, 1994; 
McKeown et al., 1997), families with a member with schizophrenia (King \& Dixon, 1995), and families with an individual with early episode psychosis (Koutra et al., 2014b). The only study to date to employ the Circumplex model in early episode psychosis examined cohesion and adaptability within Greek families and suggested that unbalanced levels of cohesion and adaptability do not trigger illness onset, but rather are outcomes of psychosis (Koutra et al., 2014b). To date, very little data exist on the structure of cohesion and adaptability in families with early episode psychosis in a large and diverse sample, and to my knowledge, no study has examined predictors of family cohesion and adaptability within this population.

\subsubsection{Strengths-Based Model of Family Resiliency}

Within the last two decades, the field of family research and family therapy has slowly undergone a paradigm shift to move away from a deficits-based approach and towards a strengths-based approach of understanding and working with families. A strength-based approach encompasses more emphasis on family resiliency, positive aspects of caregiving, and adaptive family coping strategies. Within the psychosis literature, the strengths-based movement emerged in response to previous work that had predominantly focused on concepts such as caregiver burden, expressed emotion, and maladaptive family functioning as they relate to the onset of illness and relapse. Several studies to date have shifted away from this approach and have focused instead on the interplay of family strengths and mental health. For example, studies have demonstrated that family strengths such as positive coping, active problem solving, adaptive family characteristics such as pride and cohesion, as well availability of supports and resources, 
were related to a lower duration of untreated psychosis and more treatment-seeking behaviour (e.g., Compton et al., 2009; Goulding et al., 2008; Mo’tamedi et al., 2014).

It is important to note that the family resiliency model differentiates between normative and non-normative family stressors (McCubbin et al., 1996). Normative stressors are expected life changes that many families face over their lifetime, whereas non-normative stressors are unexpected or unpredictable situational events or chronic stressors that can disrupt a family system (Lavee, McCubbin, \& Olson, 1987). The onset of a severe mental illness such as psychosis has been identified as a non-normative stressor (Saunders, 2003). The onset of a severe mental illness may pose additional challenges to the family system, wherein the family's existing strengths and coping strategies are not sufficient to resolve the difficulties that emerge in response to that stressor. Understanding the ways in which families adapt to disruptive or unexpected life changes is at the crux of family resiliency research.

\subsubsection{The Current Study}

This study aims to merge theoretical perspectives from the stress-coping model of caregiving, Circumplex model of family functioning, and strengths-based model of family resiliency. The purpose of the current study is to understand family functioning in early episode psychosis from the caregiver's perspective and to examine the factors that are associated with healthy family cohesion and adaptability during a critical period of illness. The long-term goal of this study is to contribute to a body of literature that will inform best practice family interventions and help promote healthy family functioning during this stressful time. 
There were three primary hypotheses for the current study. First, given that the onset of psychosis is a non-normative stressor that can disrupt a family system, I hypothesized that families with an individual with psychosis would report greater family difficulties compared to normative data, as measured by extreme levels of cohesion and flexibility. Second, I hypothesized that this study would replicate the stress-coping model of caregiving, such that caregiver appraisals and coping will predict caregiver quality of life over and above the contribution of the family member's level of disability and the caregiver's perceived helpfulness of support systems. Finally, I hypothesized that caregiver appraisals and coping strategies would emerge as the most relevant predictors of overall family functioning in families with an individual with early episode psychosis.

\subsection{Methods}

\subsubsection{Participants}

The inclusion criteria for this study were purposefully broad in order to capture a wide sample of caregivers and allow for the inclusion of complex or non-traditional family structures. There was only one inclusion criterion for participation in this study: participants had to be primary caregivers of an individual with early episode psychosis. Primary caregivers were defined as biological parents, foster parents, siblings, or extended family such as aunts, uncles, or grandparents who either live with or perform regular caregiver activities (e.g., groceries, household chores, scheduling appointments) for their family member with psychosis. Early episode psychosis was defined as having a first episode of psychosis within the last 5 years.

Caregivers had the option to complete the survey online or use a hard copy. Several strategies for recruitment were utilized, including posting the survey link on 
online forums, placing flyers at early psychosis intervention clinics, in-person recruitment by the primary investigator, and clinician referrals. Caregivers who participated in the online survey were recruited through various methods. Online advertisements (e.g., email listservs, message forums, Facebook) were posted with agencies such as Early Psychosis Intervention Ontario (EPION; formerly the Ontario Working Group for Early Psychosis Intervention), the Schizophrenia Society of Ontario (SSO), the Schizophrenia Society of Canada (SSC), and the National Alliance for Mental Illness (NAMI). In-person recruitment was pursued at several early psychosis intervention clinics, including the Heads Up Early Psychosis Intervention Service in Kingston, ON, the Cleghorn Early Intervention Program in Hamilton, ON, and the Lynx Early Psychosis Intervention Service in Peterborough, ON. Participants were also recruited in the United States using Amazon's Mechanical Turk (MTurk). MTurk is an online crowdsourcing service where individuals, businesses, or researchers (known as "Requesters") can post tasks for community members (known as "Workers") to access and complete. MTurk has been used in research with a variety of clinical populations and has been found to be a reliable and valid method for collecting data in clinical populations (Shapiro, Chandler, \& Mueller, 2013). Unfortunately, to date no data exist on the utility of MTurk for collecting data from caregivers of an individual with a mental illness. As such, $t$-test analyses were conducted in the present study to determine whether there were any significant differences in demographic information or family and caregiver variables between the MTurk respondents and the other online survey respondents. There were no significant differences between the groups on any of the survey measures; however, the MTurk group was younger in age and more likely to be siblings, rather than parents, of an 
individual with early psychosis. The means and standard deviations for both groups of survey respondents are included in Table 2.1.

Table 2.1.

Comparison of MTurk respondents and other online survey respondents.

\begin{tabular}{lcccc}
\hline & $\begin{array}{c}\text { MTurk } \boldsymbol{M}(\boldsymbol{S D}) \\
N=37\end{array}$ & $\begin{array}{c}\text { Other Online } \boldsymbol{M}(\boldsymbol{S D}) \\
N=65\end{array}$ & $\boldsymbol{t}$ & $\boldsymbol{p}$ \\
\hline Caregiver age & $35.43(9.59)$ & $51.92(9.36)$ & 7.94 & $<.001$ \\
Family Cohesion & $32.57(8.37)$ & $34.71(7.63)$ & 1.29 & .200 \\
Family Adaptability & $24.78(7.46)$ & $22.71(7.65)$ & -1.30 & .196 \\
ECI Negative & $1.80(0.57)$ & $1.95(0.65)$ & 1.15 & .252 \\
ECI Positive & $2.10(0.52)$ & $2.24(0.57)$ & 1.22 & .227 \\
Coping Positive & $1.71(0.50)$ & $1.84(0.42)$ & 1.33 & .186 \\
Coping Negative & $1.04(0.55)$ & $0.90(0.36)$ & -1.42 & .159 \\
Perceived Helpfulness & $2.38(1.09)$ & $2.02(0.70)$ & -1.95 & .055 \\
\hline
\end{tabular}

Note: $\mathrm{ECI}=$ Experience of Caregiving Inventory.

A power analysis for a linear multiple regression with a medium effect size, 6 predictors, alpha error probability of 0.05 , and estimated power of 0.80 yielded a required sample size of 98 participants. In total, data from 107 participants were analyzed in the present study. Participants were excluded from data analysis if they only completed the demographic section of the survey and skipped the caregiver/family questionnaires. Questionnaires that had more than 5\% missing data were excluded from analyses. In other words, if a participant only completed the demographics and one caregiver/family questionnaire (with less than 5\% missing data), then their data for those variables were 
still included in the analyses. Cases were excluded from analyses based on survey completion time and inconsistent responding, as a way to rule out invalid responses from participants that completed the survey too quickly or did not read questions carefully. The average Grade 9 reading rate is 211.64 words per minute $(S D=86.39$; Brown, Fishco, \& Hanna, 1993), therefore, I calculated the minimum survey completion time for a reading rate that was two standard deviations above the mean ( 384.42 words per minute). The total word count for the survey was 4186 words, yielding a minimum survey completion time of 10 minutes and 53 seconds. Therefore, participants who completed the survey in less than 11 minutes were excluded from analysis. There was no cutoff for maximum survey completion time as participants were allowed to save their responses and return to the survey multiple times, and I could not account for time lapses due to leaving the internet browser window open. For partially completed surveys, an individual completion time cutoff was calculated for each participant based on the number of words in the questionnaire up until the point where the participant stopped responding. Inconsistent responding on the demographic questionnaire was also used to exclude participants from analysis. Participants who reported the current age of their family member with psychosis to be lower than the age at which their family member was diagnosed were also excluded from the present study. Please refer to the consort chart in Figure 2.1 for details. Data on the differences in demographic characteristics and survey responses between participants who were included and excluded from the present study are presented in Appendix A. 
Accessed survey online

\begin{tabular}{l|c|c|}
\hline \multicolumn{2}{|c|}{$\begin{array}{c}\text { Schizophrenia } \\
\text { Society } \\
n=15\end{array}$} & NAMI \\
& $n=9$ \\
\hline
\end{tabular}

Hard copy

Flyers at early
intervention clinics
$n=14$
In-person at early intervention clinics $n=5$

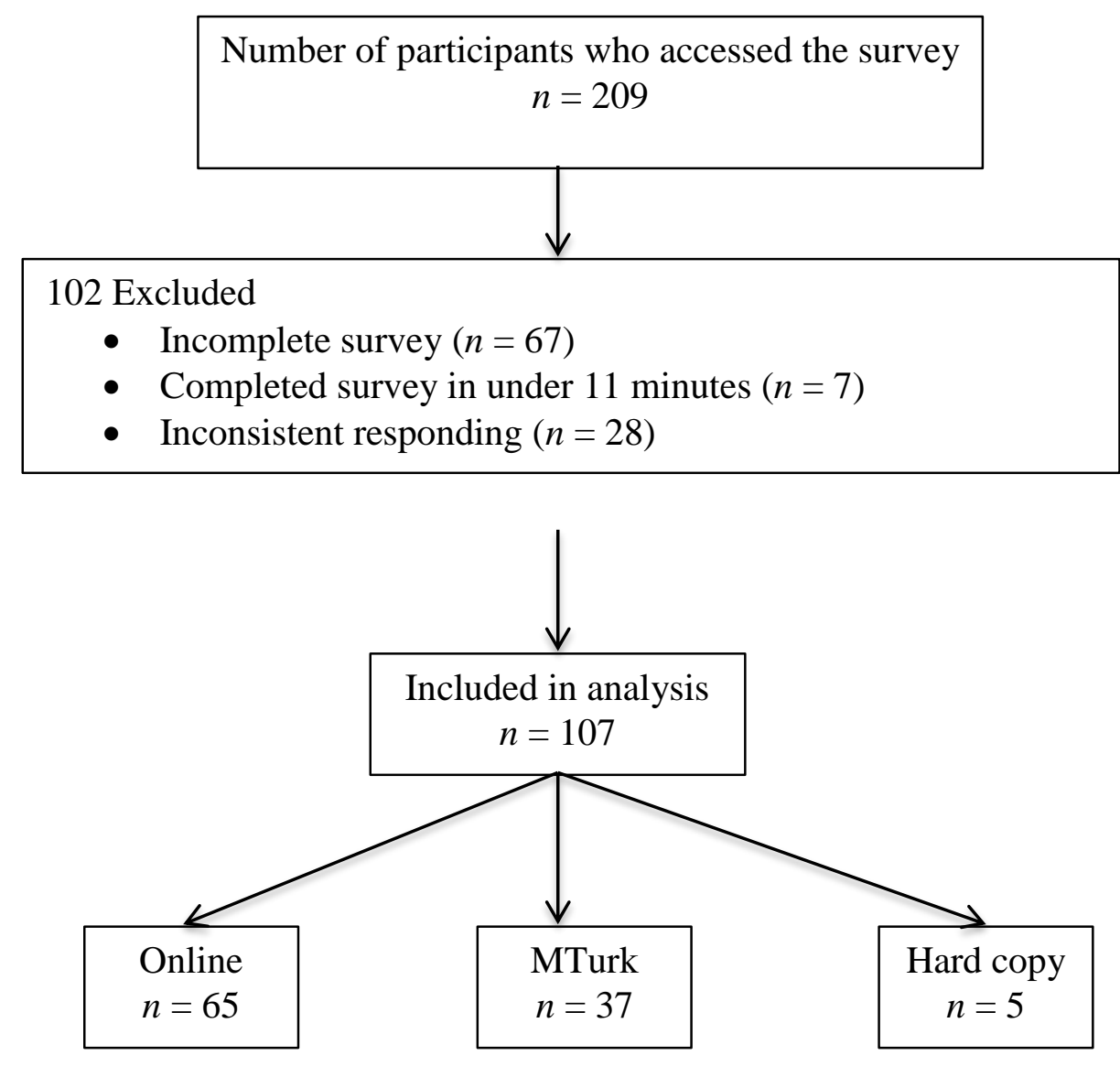

Figure 2.1. Consort chart. 
The final sample size for this study was 107 participants. Of the 107 caregivers who were included in this study, $80 \%$ were women $(n=86)$. Sixty-three percent were married $(n=67), 22 \%$ were separated or divorced $(n=23), 1 \%$ was widowed $(n=1)$, and $15 \%$ were single $(n=16)$. A diverse distribution of family caregivers participated in the study. Approximately $75 \%$ were parents or guardians $(n=80), 16 \%$ were siblings $(n=$ 17), $7 \%$ were spouses or partners $(n=7)$, and $3 \%$ were extended family $(n=3)$ of an individual with early psychosis. The modal level of education of caregivers was a university degree. Seventy-seven percent $(n=82)$ of caregivers were currently employed. Sixty-six percent $(n=71)$ of caregivers reported living with their family member with psychosis. The majority of caregivers reported that their family member with psychosis was male $(72 \%$,$) and had been hospitalized at least once for psychiatric reasons (73 \%)$. Fifty-three percent of families reported participating in family therapy at some point in their lifetime.

\subsubsection{Measures}

A summary of the participant characteristics, as well as the means and standard deviations for all survey measures is provided in Table 2.2. 
Table 2.2.

Means and standard deviations for participant characteristics and survey measures.

$\begin{array}{llll}n & M & S D & \text { Range }\end{array}$

\section{Caregiver}

Age (years)

Number of children

Family member with psychosis

Age (years)

Age of illness onset (years)

Duration psychosis was untreated (months) $\phi$

Duration in treatment (months)

Age at first psychiatric hospitalization (years)

Total number of psychiatric hospitalizations

Time elapsed since last hospitalization (months)

Caregiver survey measures

ECI Positive

Possible range $=0-4$

Higher scores indicate more positive appraisals

ECI Negative

Possible range $=0-4$

Higher scores indicate more negative appraisals

Positive Coping

Possible range $=0-3$

Higher scores indicate more positive coping

Negative Coping

Possible range $=0-3$

Higher scores indicate more negative coping

Perceived helpfulness of supports

Possible range $=1-5$

Higher scores indicate greater perceived helpfulness

$\begin{array}{rrrr}106 & 46.42 & 11.86 & 20-70 \\ 107 & 2.07 & 1.34 & 0-6\end{array}$

$107 \quad 24.36 \quad 6.48 \quad 14-42$

$\begin{array}{llll}106 & 17.84 & 6.37 & 2\end{array}$

$101 \quad 17.45 \quad 28.22 \quad 0-240$

$105 \quad 33.20 \quad 23.83 \quad 0-60$

$\begin{array}{llll}76 & 19.71 & 5.65 & 6-42\end{array}$

$\begin{array}{llll}75 & 4.08 & 5.03 & 1-30\end{array}$

$\begin{array}{llll}75 & 23.89 & 22.47 & 1-63\end{array}$

$\begin{array}{llll}106 & 2.19 & 0.55 & 0.79-3.65\end{array}$

$\begin{array}{llll}106 & 1.90 & 0.62 & 0.36-3.51\end{array}$

$\begin{array}{llll}96 & 1.80 & 0.45 & 0.88-2.63\end{array}$

$\begin{array}{llll}96 & 0.95 & 0.44 & 0.25-2.25\end{array}$

$\begin{array}{llll}98 & 2.15 & 0.87 & 0.50-4.88\end{array}$ 
Caregiver QOL

Possible range $=0-10$

Higher scores indicate greater $Q O L$

Family member with psychosis QOL

Possible range $=0-10$

Higher scores indicate greater $Q O L$
98

6.00

2.21

$0-10$

103

3.71

2.79

$0-10$

Note : ECI = Experience of Caregiving Inventory. QOL $=$ Quality of Life. $\phi$ Duration psychosis was untreated as reported by the caregiver.

2.2.2.1 Survey software. The online survey was created using Fluid Surveys and stored securely online. The following questionnaires were included in the survey.

2.2.2.2 Demographic questionnaire. The demographic questionnaire collected information such as caregiver's age, education, income, marital status, and their relationship to the family member with psychosis, as well as basic questions about family structure (e.g., number of family members in the household, frequency of contact), psychiatric history (e.g., family psychiatric history, hospitalization history, and use of hospital or community-based mental health services including family therapy).

2.2.2.3 Family functioning. This study used the Family Adaptability and Cohesion Evaluation Scale Version 3 (FACES-III; Olson, Portner, \& Lavee, 1985). The FACES-III is a 40-item self-report scale that was developed to assess two major dimensions of family functioning: family cohesion and family adaptability. Family cohesion refers to the emotional bonding that family members have toward one another. Family adaptability refers to the ability of a family system to change its power structure, roles, and relationship rules in response to situational or developmental stress.

The FACES-III is based on the Circumplex model (Olson et al., 1985) and can be used to collect self-report data on family cohesion and adaptability categorically, for example the four levels of cohesion include (1) disengaged, (2) separated, (3) connected, 
and (4) enmeshed, and the four levels of adaptability include (1) rigid, (2) structured, (3) flexible, and (4) chaotic. Participants are asked to describe their family functioning on a 5-point Likert-type scale with items ranging from 0 (almost never) to 4 (almost always). The Circumplex model postulates a curvilinear relationship, wherein both extremely high and extremely low levels of cohesion and adaptability are maladaptive, and balanced levels of these family dimensions are adaptive. A continuous score is calculated as the "distance from center", which indicates the quantitative distance of an individual's cohesion and adaptability scores from the center of the Circumplex model.

Normed data for the FACES-III is provided for parents across all stages of the life cycle, families with adolescents, and couples without children. The internal consistency of the scale in the present study was very good with a Cronbach's alpha of 0.89 .

2.2.2.4 Perceived helpfulness of available support systems. Information about caregivers' informal and formal social support systems was collected using the Family Support Scale (FSS; Dunst, Trivette, \& Hamby, 1994). The FSS measures the use and perceived helpfulness of 18 types of informal (e.g., spouse, friends, coworkers, church) and formal (e.g., agencies, professionals) supports used in the last six months. The items included yes/no answers for use of these various support systems and perceived helpfulness was rated on scale ranging from 1 (not at all helpful) to 5 (extremely helpful), where higher scores indicated greater perceived helpfulness. Participants also had the option to select NA (not available). In the current study, the FSS demonstrated good internal consistency with a Cronbach's alpha of 0.85 .

2.2.2.5 Experience of caregiving. The Experience of Caregiving Inventory (ECI; Szmukler et al., 1996) is a 66-item self-report measure of the experience of caregiving for 
a person with a severe mental illness. In essence, the ECI is a measure of caregiver appraisal. Previous research indicated that caregiver wellbeing is the result of an interaction between the caregiver's appraisal of their role and his or her own coping strategies (Joyce et al., 2000; Szmukler et al., 1996). The caregiver's appraisal is affected both by the demands or stressors that result from their caregiving role, (e.g., family member's symptom severity or level of functional disability), as well as environmental factors such as social support. The ECI consists of ten subscales, eight negative (difficult behaviours; negative symptoms; stigma; problems with services; effects on family; need to backup; dependency; loss) and two positive (rewarding personal experiences; good aspects of relationship with the patient). The ECI asks caregivers how often they have had certain thoughts in the past month, and the items range from 0 (never) to 4 (nearly always). Higher scores on the ECI Negative Subscale indicate greater negative appraisals and higher scores on the ECI Positive Subscale indicate greater positive appraisals. Previous studies have shown that the ECI has acceptable validity, as the results were associated with a stress-coping model of caregiving (Joyce et al., 2000; Szmukler et al., 1996). In the present study, the ECI displayed excellent internal consistency, with a total scale Cronbach's alpha of 0.95. The Cronbach's alpha for the ECI Positive subscale was 0.81 , and 0.75 for the ECI Negative subscale.

2.2.2.6 Coping skills. The Brief COPE (Carver, 1997) is a 28 -item coping response scale that captured 14 coping strategies. These responses can be broadly classified as either adaptive: (1) active coping, (2) planning, (3) positive reframing, (4) acceptance, (5) humour, (6) religion, (7) using emotional support from others, (8) using instrumental support, such as asking for advice from others, or maladaptive: (9) self- 
distraction, (10) denial, (11) venting, (12) substance use, (13) behavioural disengagement, and (14) self-blame. Responses were made on a 4-point scale ranging from 1 (I haven't been doing this at all) to 4 (I've been doing this a lot). The internal consistency of the total scale revealed a Cronbach's alpha of 0.76. The Cronbach's alpha for the positive coping subscale was 0.75 and the Cronbach's alpha for the negative coping scale was 0.72 . This scale has been used in numerous health-related studies, including drug addiction, aging, cancer, AIDS, and depression. It has also been used in studies of individuals with severe mental illness (Meyer, 2001), early episode psychosis, and ultra-high risk for psychosis (Pruessner et al., 2011), as well as caregivers of individuals with psychosis (Fortune, Smith, \& Garvey, 2005).

2.2.2.7 Daily functioning/quality of life. The Sheehan Disability Scale (Sheehan, 2000) is a 3-item rating of a patient's global functioning and satisfaction in three domains: (1) Work/ School, (2) Social Life, and (3) Family Life/ Home Responsibilities. The Sheehan Disability Scale was rated on a 10-point scale. Each caregiver completed the Sheehan Disability Scale twice, once as a self-report measure for himself or herself and once as a third-party report for the family member. The internal consistency for the Sheehan Disability Scale was good, with a Cronbach's alpha of 0.80 for self-report and 0.86 for third-party reports of the family member with psychosis.

\subsubsection{Procedure}

Data were collected online via a survey link that was distributed to early intervention in psychosis clinics across Canada and the United States. The survey link was also posted on online forums, such as EPION, SSO, SSC, and NAMI, as well as to the general community via MTurk. Survey links were also presented by a flyer, in-person 
to the caregiver by the primary investigator, or by clinicians at early intervention clinics who were provided with a brief clinician information sheet and asked to give a pamphlet to caregivers on their caseload.

When recruitment was done in-person by the primary investigator, potential participants were brought to a private room (e.g., an office) to discuss the study. The primary investigator relayed the study aims so as to emphasize that the study is interested in learning about the positive ways in which families help to support one another during the recovery process (and not to perpetuate negative views or stereotypes about parentblaming).

For each caregiver that completed the survey, a donation of $\$ 10$ was made to the Schizophrenia Society of Canada. For caregivers who consented, a brief report was written up for the family worker at the Heads Up Early Psychosis Intervention Service to provide clinically meaningful information to use in individual caregiver support sessions.

\subsubsection{Data Analyses}

A scatterplot of cohesion and adaptability was conducted in order to provide a visual illustration of the distribution of family structures across the various categories of family cohesion and flexibility. Chi-square analyses were conducted for family structures

of cohesion (disengaged, separated, connected, enmeshed) adaptability (rigid, structured, flexible, chaotic), and global family functioning (extreme difficulties, mid-range, and balanced) in families with an individual with early episode psychosis compared to existing normative data on adult families from the FACES-III administration and scoring manual (Olson et al., 1985). These normative data were collected on 2,453 adult parents across the family life span. The categories of cohesion, adaptability, and global family 
functioning were based on cut-off points from the FACES-III manual. The FACES-III also provides a variable called the Distance from Center (DFC), which indicates the quantitative distance of an individual's cohesion and adaptability scores from the center of the Circumplex model. The DFC is calculated using the following formula, where 39.8 is the mean total cohesion score and 24.1 is the mean total adaptability score based on existing normative data:

$\mathrm{DFC}=\sqrt{(\text { Total cohesion score }-39.8)^{2}+(\text { Total adaptability score }-24.1)^{2}}$

Correlations were conducted to examine the relationships among youth clinical variables, caregiver appraisals, caregiver coping strategies, perceived helpfulness of support systems, quality of life, and family functioning. The Holm's step-down correction was used to control for family-wise error. This procedure involves sorting $p$ values from lowest to highest, and then multiplying the $p$-values by sequentially decreasing number of comparisons. For example, the lowest $p$-value is multiplied by the total number of comparisons $(n)$, and this adjusted $p$-value is compared to an alpha of .05 . The second lowest $p$-value is then multiplied by $n-1$ and compared to an alpha of .05 . The third lowest $p$-value is multiplied by $n-2$ and compared to .05 . This sequential procedure continues for all comparisons and only adjusted $p$-values that are below .05 are statistically significant. The Holm step-down procedure has been considered superior to the Bonferonni correction because it is conservative, yet more powerful (Aickin \& Gensler, 1996). It is also the most optimal correction among other step-down procedures (Gordon \& Salzman, 2008). 
A forced-entry hierarchical linear regression was used to replicate the stresscoping model of caregiving (Addington et al., 2003; Joyce et al., 2003; Szmukler et al., 1996). The dependent variable in the regression was caregiver quality of life, and the independent variables were entered in the following order: Step 1 = youth global functioning, Step 2 = caregiver perceived helpfulness of supports, and Step $3=$ positive caregiver appraisals, negative caregiver appraisals, positive coping strategies, negative coping strategies. The order of variables in each step was selected based on the stresscoping model of caregiver wellbeing, wherein support systems produce added predictive value above illness demands, and appraisals and coping strategies produce added predictive value above and beyond both illness demands and social support (Addington et al., 2003; Joyce et al., 2003; Szmukler et al., 1996).

Finally, an exploratory stepwise linear regression was conducted to determine if variables related to the stress-coping model of caregiving were also associated with family functioning. The analysis is exploratory as this is the first study to examine predictors of family cohesion and adaptability in early episode psychosis and a strong theoretical model has not yet been built. The measure of family of functioning, "distance from center" of the family Circumplex model was used as the dependent variable in the regression. The independent variables were based on previous literature in support of a caregiver stress-coping model to determine if a similar stress-coping model can be applied to family functioning. The predictors were: positive caregiver appraisals, negative caregiver appraisals, use of positive coping strategies, use of negative coping strategies, and perceived helpfulness of available support systems. 


\subsection{Results}

\subsubsection{Family Cohesion and Adaptability Scatterplot}

A scatterplot was created in order to illustrate the distribution of family cohesion and adaptability in the current sample. Each participant's total score for cohesion was plotted along the $x$-axis and the total score for adaptability was plotted along the $y$-axis. The total sum for the cohesion subscale was significantly correlated with the total sum for the adaptability subscale, $r(100)=.24, p=.016$. The Circumplex model (Olson et al., 1985) was superimposed over the scatterplot in order to visually illustrate the quadrants of both dimensions of cohesion and adaptability (refer to Figure 2.2).

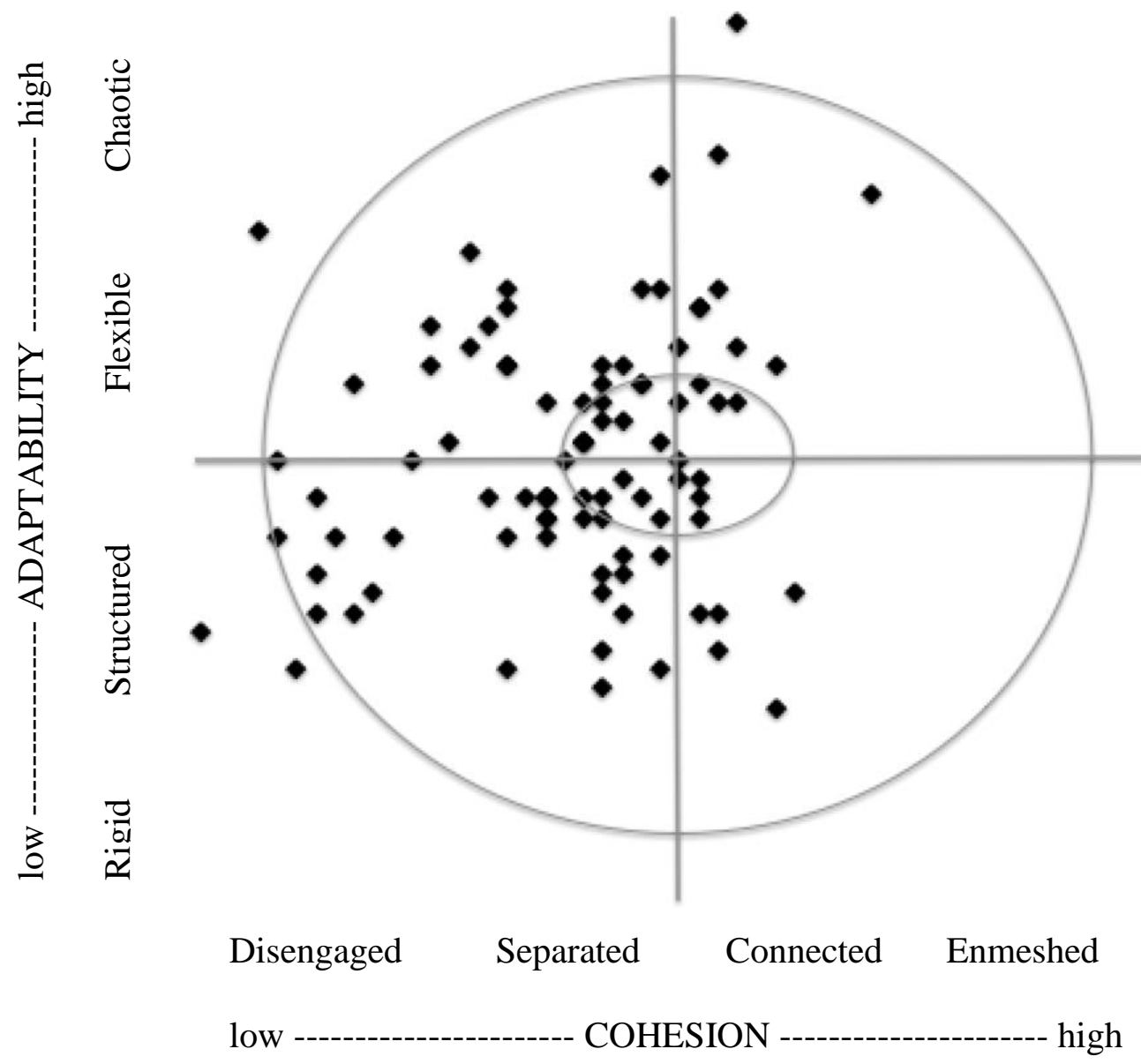

Figure 2.2.

Scatterplot of cohesion and adaptability scores. 


\subsubsection{Chi-Square Analysis}

The frequencies of cohesive and adaptable family structures were compared to normative data from the FACES-III manual. A two-way chi-square analysis was conducted to evaluate whether family structures from the current study were significantly different from existing normative data on 2,453 adult families (Olson et al., 1985). Expected frequencies were calculated using the manual's normative data. The manual reported the percentage of families that fell into each of the eight family structure categories (4 levels of cohesion and 4 levels of adaptability). Expected frequencies were then calculated by multiplying the percentage value by the current sample size to get an estimate of the expected numbers of families in each group (e.g., the percent of families who were "disengaged" in the normative data was $16.3 \%$. Therefore, 0.163 was multiplied by the total number of participants who completed the FACES-III, $n=100$, and yielded an expected frequency of 16.3 disengaged families within the current sample).

The chi-square was significant for the dimension of cohesion, $\chi^{2}(3)=57.35, p<$ .001. The observed and expected proportions across the four levels of cohesion (disengaged, separated, connected, and enmeshed) are displayed in Table 2.3. It is important to keep in mind that extreme levels of cohesion are maladaptive, such that both the "disengaged" and "enmeshed" categories are indicative of maladaptive levels of cohesion, and mid-level categories of "separated" and "connected" are indicative of balanced and healthy family cohesion. Follow-up chi-square analyses could not be conducted given that raw data on healthy controls were not collected as part of this study, but rather data on families with an individual with psychosis were compared to normative 
data from the FACES-III manual. In general, families with an individual with early psychosis who participated in the current study were more disengaged, more separated, less connected, and less enmeshed compared to the normative data.

Table 2.3.

Observed and expected frequencies of families as a function of type of cohesion.

\begin{tabular}{lll}
\hline & Observed & Expected \\
\hline Disengaged & 41 & 16.3 \\
Separated & 39 & 33.8 \\
Connected & 18 & 36.3 \\
Enmeshed & 2 & 13.6 \\
Total & 100 & \\
\hline
\end{tabular}

The chi-square was also significant for the dimension of adaptability, $\chi^{2}(3)=$ $16.30, p=.001$. The observed and expected proportions across the four levels of adaptability (rigid, structured, flexible, and chaotic) are displayed in Table 2.4. Again, extreme levels of adaptability are maladaptive, such that both the "rigid" and "chaotic" categories are indicative of maladaptive levels of adaptability, and mid-level categories of "structured" and "flexible" are indicative of balanced and healthy family adaptability. Families with an individual with early psychosis who participated in the current study were generally more rigid, less structured, less flexible, and more chaotic compared to the normative data.

Table 2.4.

Observed and expected frequencies of families as a function of type of adaptability.

\begin{tabular}{lll}
\hline & Observed & Expected \\
\hline Rigid & 26 & 16.2 \\
Structured & 29 & 38.4 \\
Flexible & 20 & 29.4 \\
Chaotic & 25 & 16.0 \\
Total & 100 & \\
\hline
\end{tabular}


A chi-square analysis was also conducted for global family functioning. The global family functioning variable combines the dimensions of cohesion and adaptability to categorize families as having "extreme difficulties" (i.e., the extreme and outer circle of the Circumplex model), "mid-range difficulties", or "balanced" (i.e., the inner circle of the Circumplex model where families exhibit balanced levels of both cohesion and adaptability). The chi-square was significant for global family functioning, $\chi^{2}(2)=22.01$, $p<.001$. The observed and expected proportions across the three levels of family functioning are displayed in Table 2.5. Families with an individual with early episode psychosis generally reported less balanced family systems, as well as more extreme difficulties and mid-range difficulties, relative to normative data.

Table 2.5.

Observed and expected frequencies of global family

functioning.

\begin{tabular}{lll}
\hline & Observed & Expected \\
\hline Balanced & 28 & 48.6 \\
Mid-Range & 50 & 40.4 \\
Extreme & 22 & 11.0 \\
Total & 100 & \\
\hline
\end{tabular}

\subsubsection{Correlations}

I analyzed Pearson's $r$ correlations between the means for the youth clinical variables, difficulties with family functioning, self-reported caregiver appraisals (both positive and negative appraisals about the caregiving role, the demands of the illness, and its consequences), use of coping strategies, perceived helpfulness of support systems, quality of life, and difficulties with family functioning as calculated by the "distance from center" (DFC) in the Circumplex model. 
The Pearson's $r$ correlation coefficients for the youth clinical variables and caregiver variables related to family difficulties (DFC) are presented in Table 2.6. Only positive caregiver appraisals were significantly correlated with DFC at the 0.05 level, $r$ $(99)=-.26 p=.009$, such that greater positive caregiver appraisals were associated with more adaptive family functioning. However, it is important to note that this correlation does not maintain significance when a Holm step-down correction to control family-wise error rate is applied.

Table 2.6.

Pearson's $r$ correlation coefficients of the "distance from center" of the Circumplex model with youth clinical variables and caregiver variables.

\begin{tabular}{ll}
\hline & DFC \\
\hline Age of illness onset & -.14 \\
Duration illness was untreated & .16 \\
Number of psychiatric hospitalizations & .10 \\
Duration since last hospitalization & .01 \\
Positive appraisals & $-.26^{*}$ \\
Negative appraisals & .07 \\
Positive coping & .03 \\
Negative coping & .08 \\
Caregiver quality of life & -.17 \\
Youth quality of life & -.16 \\
Perceived helpfulness & -.17 \\
\hline
\end{tabular}

Notes: *indicates $p<.05$ level (2-tailed), however this statistic does not maintain significance after applying a Holm step-down procedure to control for family-wise error.

Pearson's $r$ correlations were also conducted to determine the association among the means of the youth clinical variables, caregiver appraisals, coping, perceived helpfulness of supports, as well as caregiver and youth quality of life. The results of the correlational analysis are summarized in Table 2.7. After applying Holm step-down correction to control for family-wise error, the correlations that remained significant were (1) the association between greater negative caregiver appraisals and lower caregiver 
quality of life, $r(97)=-.49, p<.001$, (2) the association between greater negative caregiver appraisals and lower youth quality of life, $r(102)=-.62, p<.001$ (3) the association between greater use of positive coping strategies and greater perceived helpfulness of support systems, $r(95)=.35, p<.001$, (4) the association between greater use of positive coping strategies and better caregiver quality of life, $r(96)=.41, p<.001$, (5) greater perceived helpfulness of supports and better caregiver quality of life, $r(98)=$ $.42, p<.001,(6)$ greater perceived helpfulness of supports and better youth quality of life, $r(97)=.42, p<.001$, and (7) greater caregiver quality of life and greater youth quality of life, $r(98)=.48, p<.001$. 
Table 2.7.

Pearson's r correlation coefficients among youth clinical variables, caregiver appraisals, coping, perceived helpfulness of supports, and caregiver and youth quality of life.

\begin{tabular}{|c|c|c|c|c|c|c|c|c|c|c|}
\hline & $\begin{array}{l}\text { Duration } \\
\text { untreated } \\
\text { illness }\end{array}$ & $\begin{array}{l}\text { Number } \\
\text { of hosp. }\end{array}$ & $\begin{array}{l}\text { Time since } \\
\text { last hosp. }\end{array}$ & $\begin{array}{l}\text { Positive } \\
\text { appraisals }\end{array}$ & $\begin{array}{l}\text { Negative } \\
\text { appraisals }\end{array}$ & $\begin{array}{l}\text { Positive } \\
\text { coping }\end{array}$ & $\begin{array}{l}\text { Negative } \\
\text { coping }\end{array}$ & $\begin{array}{l}\text { Caregiver } \\
\text { QOL }\end{array}$ & $\begin{array}{l}\text { Youth } \\
\text { QOL }\end{array}$ & $\begin{array}{l}\text { Perceived } \\
\text { helpfulness }\end{array}$ \\
\hline $\begin{array}{l}\text { Age of illness } \\
\text { onset }\end{array}$ & .07 & .01 & .04 & -.13 & .10 & $-.24 *$ & -.11 & -.18 & $-.21 *$ & $-.31 * *$ \\
\hline $\begin{array}{l}\text { Duration } \\
\text { untreated illness }\end{array}$ & & .21 & .12 & -.11 & .01 & .05 & $-.21 *$ & .06 & -.17 & -.09 \\
\hline Number of hosp. & & & $-.28 *$ & -.18 & -.01 & .22 & .05 & -.12 & -.22 & -.07 \\
\hline $\begin{array}{l}\text { Time since last } \\
\text { hosp. }\end{array}$ & & & & .12 & $-.30 *$ & -.03 & -.19 & $.30 *$ & $.29 *$ & -.07 \\
\hline $\begin{array}{l}\text { Positive } \\
\text { appraisals }\end{array}$ & & & & & .17 & .05 & -.07 & .07 & .18 & .10 \\
\hline $\begin{array}{l}\text { Negative } \\
\text { appraisals }\end{array}$ & & & & & & -.18 & $.22 *$ & $-.49 * *$ & $-.62 * *$ & $-.32 * *$ \\
\hline Positive coping & & & & & & & -.01 & $.41 * *$ & .12 & $.35 * *$ \\
\hline Negative coping & & & & & & & & -.20 & .06 & .16 \\
\hline Caregiver QOL & & & & & & & & & $.42 * *$ & $.42 * *$ \\
\hline Youth QOL & & & & & & & & & & $.48 * *$ \\
\hline
\end{tabular}

Notes: Values represent Pearson's r correlation coefficients rounded to the second decimal place. The sample size varies slightly depending on the variables included within each correlation. * indicates significance at the $p<.05$ level (2-tailed). ** indicates significance at the $p<.01$ level (2tailed). 


\subsubsection{Hierarchical Linear Regression}

A forced-entry hierarchical linear regression was conducted to replicate the stresscoping model of caregiving (Addington et al., 2003; Joyce et al., 2003; Szmukler et al., 1996). The dependent variable was caregiver quality of life. The independent variables were entered in the following steps: Step $1=$ patient level of functioning and disability (Sheehan Disability Scale), Step 2 = perceived helpfulness of supports (Family Support Scale), Step 3 = positive and negative caregiver appraisals (Experience of Caregiving Inventory) and positive and negative coping strategies (Brief Cope). The full regression model was statistically significant, $F(6,88)=10.43, p<.001$, with better caregiver quality of life being predicted by better patient functioning in the first step $\left(\mathrm{R}^{2} \Delta=.20\right)$, greater perceived helpfulness of supports in the second step $\left(\mathrm{R}^{2} \Delta=.05\right)$, and fewer negative appraisals, fewer negative coping strategies, greater positive appraisals, and greater positive coping strategies in the third step $\left(\mathrm{R}^{2} \Delta=.17\right)$. Please refer to Table 2.8. for the regression model coefficients of each step.

A follow-up Fisher's $r$ to $z$ test was conducted to determine the difference between the $\boldsymbol{\beta}$ coefficients of positive appraisals and negative appraisals, as well as between positive coping and negative coping from the caregiver QOL regression analysis. The magnitude of the coefficient for negative appraisals is statistically significantly different than that of positive appraisals $(z=2.21, p=.027)$, and the magnitude of the coefficient for positive coping is statistically significantly different than that of negative coping $(z=3.03, p=.002)$. 
Table 2.8.

Predictors of better caregiver quality of life.

\begin{tabular}{clcccc}
\hline Model & & $\boldsymbol{\beta}$ & $\boldsymbol{t}$ & $\boldsymbol{p}$ & $\boldsymbol{R}^{2} \boldsymbol{\Delta}$ \\
\hline 1 & Patient disability & .45 & 4.8 & $<.001$ & .20 \\
\hline 2 & Patient disability & .32 & 3.0 & .004 & .05 \\
& Perceived helpfulness of supports & .25 & 2.4 & .019 & \\
\hline 3 & Patient disability & .11 & .87 & .389 & .17 \\
& Perceived helpfulness of supports & .20 & 1.9 & .057 & \\
& Negative appraisals & -.27 & -2.22 & .029 & \\
& Negative coping & -.17 & -1.97 & .053 & \\
& Positive appraisals & .05 & .54 & .588 & \\
& Positive coping & .27 & 3.05 & .003 & \\
\hline
\end{tabular}

\subsubsection{Stepwise Linear Regression}

A stepwise linear regression was conducted to determine the association between family functioning and various caregiver factors including positive caregiver appraisals, negative caregiver appraisals, positive coping, negative coping, and perceived helpfulness of supports. Family functioning was calculated as the "distance from center" (DFC) in the Circumplex model, and as such higher DFC scores are indicative of more extreme or unbalanced levels of cohesion and adaptability. The regression model was statistically significant, $F(1,92)=8.59, p=.004$. The only predictor variable that was retained in the regression model was positive caregiver appraisals, such that more positive appraisals of the illness and one's caregiving role was associated with better family functioning, $\beta=$ $-.29, t=-2.73, p=.004, \mathrm{R}^{2}=0.09$. Please refer to Figure 2.3 for a scatterplot of the relationship between positive caregiver appraisals and family difficulties as measured by DFC. 


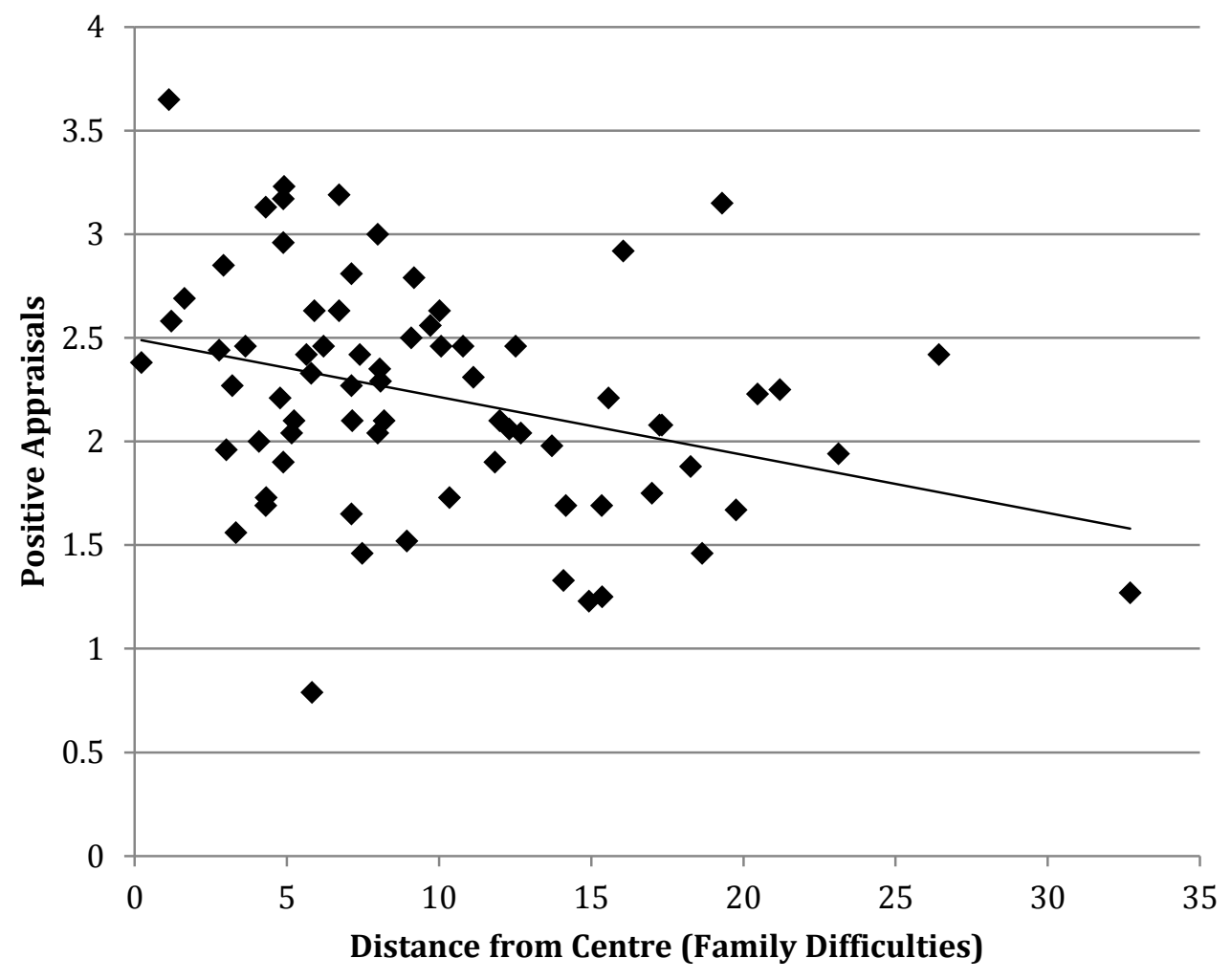

Figure 2.3. Scatterplot of caregiver positive appraisals and family difficulties.

Given the important relationship between positive caregiver appraisals and family functioning, a bivariate correlational analysis was conducted between family difficulties and the two subscales that comprise the domain of positive appraisals. Difficulties with family functioning, as measured by DFC, was significantly inversely correlated with the subscale of Positive Relationships $r(99)=-.29, p=.004$, but not with the subscale of positive Personal Experiences, $r(99)=-.15, p=.125$. This indicates that positive appraisals about one's relationship with the affected family member (e.g., "I feel useful in my relationship with him", "He has shown strengths in coping with his illness") may be driving the relationship between positive appraisals and better family functioning relative 
to positive appraisals about one's personal caregiving experiences (e.g., "I have learnt more about myself", "I have discovered strengths in myself").

\subsection{Discussion}

The purpose of this study was to examine differences in family cohesion and flexibility in families with an individual with early episode psychosis, and to assess the relationships among caregiver appraisals, coping strategies, perceived helpfulness of support systems, and family functioning. There were three primary hypotheses: (1) that families with an individual with psychosis will exhibit greater family difficulties, such as extreme levels of cohesion and flexibility, (2) that caregiver appraisals and coping strategies would predict caregiver quality of life above and beyond patient disability and perceived helpfulness of support systems, and (3) that caregiver appraisals and coping strategies would emerge as the most relevant predictors of adaptive family functioning.

The first hypothesis, that families with an individual with psychosis would experience more family difficulties, was borne from the family resiliency model wherein the onset of a severe mental illness, such as psychosis, is considered a non-normative stressor that can disrupt a family system. Families with an individual with early psychosis must face many challenges, including understanding the symptoms of the illness, accessing health care services, and managing the stress and trauma of a hospitalization (Addington et al., 2003; Barker et al., 2001; Gerson et al., 2009; Jones, 2009; Knock et al., 2011; McCann et al., 2011; Onwumere, Bebbington, \& Kuipers, 2011). As such, these family systems may be more vulnerable to disruption and more likely to experience difficulties with family functioning. The results of the present study supported the hypothesis that families with an individual with psychosis experience greater challenges 
with everyday family functioning. Compared to normative data, family members of an individual with psychosis were more likely to report extreme family difficulties such as disengagement, rigidity, and chaos compared to normative data from healthy control families.

Correlational analyses were conducted to determine relationships across a range of clinical, caregiver, and family variables. Statistically significant associations were observed between caregivers' own negative cognitions about the illness and reduced satisfaction with work, family, and social life, both for the caregiver and the individual with psychosis. Frequent use of healthy and positive coping strategies was linked to a greater likelihood of perceiving other people and agencies as being helpful resources. Furthermore, caregivers who more frequently used positive coping strategies were also likely to experience increased satisfaction with work, family, and social life. Finally, caregivers' perception that other people and agencies are helpful resources was associated with greater satisfaction with work, family, and social life for both the caregiver and the individual with early episode psychosis. Although these associations shed light on some interesting relationships between potentially malleable treatment targets (i.e., cognitive appraisals, behavioural coping strategies, attitudes related to the helpfulness of support systems), with outcome variables such as caregiver quality of life and patient quality of life, it is important to exercise caution in interpreting correlational findings. Many of these relationships could be bi-directional or influenced by other factors that have not been measured in this study.

The findings from the present study provided support for the stress-coping model of caregiving. In line with previous research, caregivers' cognitive appraisals about the 
impact of the illness and its consequences, as well as the behavioural coping strategies they employed, were strongly associated with caregiver quality of life above and beyond the level of disability of their affected family member and the perceived helpfulness of available support systems. These findings contribute to an expanding body of literature highlighting the importance of caregiver support and caregiver self-care as part of early intervention services. People have the capacity to experience simultaneously positive and negative emotions (Larsen, McGraw, \& Cacioppo, 2001); therefore caregivers of an individual with psychosis may experience both positive and negative appraisals about the illness and their caregiving role. Interestingly, negative appraisals appeared to have a greater association with caregiver quality of life than positive appraisals, suggesting that negative cognitions related to the illness, its consequences, and one's own caregiving role can have a detrimental impact on caregiver quality of life despite the presence of positive appraisals. However, the behavioural implementation of positive coping strategies offers a potential protective effect on caregiver quality of life. Although negative appraisals may be an unavoidable part of any challenging role such as caretaking, interventions aimed at the development of positive coping strategies may be promising treatment targets for family members of an individual with early psychosis.

The results from the present study provided partial support for the hypothesis that similar to a stress-coping model of caregiving, appraisals and coping strategies are also strongly associated with family functioning. Analyses revealed that positive caregiver appraisals were significantly associated with family functioning. Other caregiver variables, such as positive coping strategies, negative coping strategies, negative caregiver appraisals, and perceived helpfulness of available support systems, were not 
related to family functioning. Interestingly, greater positive appraisals, but not necessarily fewer negative appraisals, were predictive of adaptive family functioning. This finding underscores the importance of fostering positive appraisals in family members. Positive caregiver appraisals, such as a sense of pride and increased closeness to the affected family member are critical components of adaptive family functioning. Taken together, the findings from both regression analyses suggest that although negative appraisals are detrimental to caregiver wellbeing (despite the presence of positive appraisals), it is positive appraisals that are most important for family wellbeing.

This study has important implications for family work in early episode psychosis. Caregiving for a family member with psychosis can be a stressful, emotionally taxing, and frightening task (Addington et al., 2003; Barker et al., 2001; Gerson et al., 2009; Jones, 2009; Knock et al., 2011; McCann et al., 2011); as such caregivers will experience some negative appraisals about their caregiving role. Human beings have a great propensity to experience both positive and negative emotions simultaneously (Larsen et al., 2001), therefore negative appraisals related to the cost of illness in a family member, its consequences for that individual and oneself, and the challenges of caregiving are not surprising. Although negative appraisals are strongly related to the caregiver's quality of life, the use of positive coping strategies may offer some protection from the impact of these negative appraisals. On the other hand it is the presence of positive appraisals (and not the absence of negative appraisals) that is most related to overall family wellbeing. Clinically, this suggests differential treatment targets based on the goals of intervention. Promoting healthy behavioural coping strategies through individual therapy may be important to preserving caregiver wellbeing during an undoubtedly stressful period, 
whereas developing positive appraisals about illness in the family may be a critical treatment target for a system-based family intervention.

It is important to consider several limitations when interpreting these results. First, the normative data used in the chi-square analyses were derived from the FACESIII instructor manual (Olson et al., 1985), which was published over 30 years ago. As such, the normative data may not represent family functioning in the present day. Although the updated FACES-IV (Olson, 2011) is available, it was not used in the current study given that limited validation data were published at the time of data collection. It is also important to note that the use of the FACES as a measure of family functioning has both advantages and limitations. Its distinct advantage is that it measures family functioning along two dimensions, cohesion and flexibility, rather than a single construct such as expressed emotion. However, it is limited by nature of being a selfreport measure, which can be influenced by a multitude of factors including bias, social desirability, and memory deficits, to name a few. There remains a strong need for the use objective measures of family functioning that are not biased by self-report. Furthermore, in the present study the FACES-III was completed by a family member of an individual with psychosis and therefore represents that individual's unique perception of general family functioning. It is possible, and quite likely, that different family members from the same family unit would report varying degrees of cohesion and flexibility. The implementation of this tool across multiple family members, including the family member affected by psychosis, would be an important future direction for researchers interested in examining family functioning from a systems perspective. 
To my knowledge, this is the first paper to examine predictors of family cohesion and flexibility in early episode psychosis, and in many ways, this study raises more questions than it answers. Positive caregiver appraisals emerged as the only significant predictor of family functioning, however, it only accounted for $9 \%$ of the variance. Clearly, there are other variables not measured in the present study that account for a substantial portion of family functioning in this population. An important next step that would build upon the foundation of this study would be to collect information from multiple family members in order to gather a more comprehensive landscape of family functioning from each individual within a family system. In addition, other studies offer some clues as to potential variables of interest for future research in this area. For example, metacognition may be related to caregiving experiences (Jansen et al., 2014). Metacognition is the capacity to understand one's own thought processes. Jansen and colleagues found that while higher levels of metacognition were not associated with less negative caregiver experiences, they were associated with greater positive caregiver experiences. The authors concluded that although greater awareness of and capacity for complex ideas may not reduce distress, it might broaden one's ability to appreciate both positive and negative caregiver experiences. Metacognition, in all family members, may be an important contributor to family functioning. Another variable of interest for caregiver wellbeing and family functioning is hope. Hopefulness may be a critical factor in a family's ability to cope with the impact of a severe mental illness such as psychosis (Bland \& Darlington, 2002). Hope may be particularly important in fostering positive caregiver appraisals, which could then translate to more adaptive family functioning. This may be especially relevant in the context of accessing early intervention services, 
wherein health professionals may play a role in sustaining or diminishing a family's hopefulness (Bland \& Darlington, 2002). Finally, a recent review identified protective factors that may help to foster family resiliency in general. The authors identified protective factors across multiple levels, including individual, family, and community levels (Benzies \& Mychasiuk, 2009). Individual factors that related to family resiliency were: locus of control, emotional regulation, belief systems, self-efficacy, effective coping, increased education, health, temperament, and being of female gender. Family factors included: family structure (i.e., smaller families and mature parents), intimate partner relationship stability, family cohesion, supportive parent-child interactions, stimulating learning environments, social support, stable income, and adequate housing. Community-level factors that were important for family resiliency included: involvement in the community, peer acceptance among children, supportive mentors for children, safe neighborhoods, access to quality schools, and access to quality health care. Understanding family functioning and resiliency is an unquestionably complex task, wherein multiple factors across individual and system levels interact to promote or disrupt family functioning.

Family functioning is a complex and ever-evolving construct. However, despite its complexity, developing an empirical understanding of the factors that initiate and maintain adaptive family functioning in the face of illness and stress is an important research goal for the field of early psychosis intervention. This study provides a platform from which we can refine our understanding of family functioning in early episode psychosis and inform important treatment targets. Although treatment targets aimed at improving patient outcomes and caregiver outcomes are well established in the literature, 
differential treatment targets may emerge for systems-based family therapy in psychosis. Although positive appraisals are a promising treatment target, we still have a long way to go in understanding the set of factors, and their interactions, that are most critical for fostering healthy family functioning and promoting recovery from psychosis for the whole family. 


\section{Chapter 3}

\section{Application of a Behavioural Paradigm to Understanding Family Functioning in Early Episode Psychosis}

\subsection{Introduction}

\subsubsection{Family Flexibility and Resilience}

For several decades, the construct of family flexibility has been at the forefront of family research and family therapy. Family flexibility can be defined as the ability of the family system to alter its power structure, roles, and relationship rules in response to situational and developmental stress (Olson, Sprenkle, \& Russel, 1979; Olson et al., 2000). This definition overlaps considerably with the notion of family resilience. The family resiliency model (McCubbin \& McCubbin, 1993; McCubbin, Thompson, \& McCubbin, 1996) is a theoretical approach to research wherein family resilience refers to the dimensions or properties of a family that allow it to adapt to disruptive life changes or crisis events.

The family resiliency model proposes two types of family stressors: normative and non-normative (McCubbin et al., 1996). Normative stressors are expected life changes that most families face over their lifetime, and as such do not pose high risk to the disruption of a family system. On the other hand, non-normative stressors are unexpected and unpredictable situational events (e.g., natural disaster, war, death of a family member) or persistent stressors that bring long-term demands (e.g., chronic illness, unemployment) that pose a risk to a family system (Lavee et al., 1987). The family resiliency model further suggests that in the face of a normative stressor, families 
often adjust to the change using their existing strengths. However, non-normative stressors may pose additional challenges in which the existing strengths of the family are not sufficient to resolve the difficulties that emerge in response to that stressor. In order to overcome the non-normative stressor, families often need to engage in a process of adaptation in which they alter their family patterns. The onset of a severe mental illness, such as psychosis, has been identified as a potential non-normative stressor for a family system (Saunders, 2003).

The concept of family flexibility is particularly interesting in the context of psychosis. Many people with a psychotic disorder experience difficulties with flexibility across an array of psychological, neurocognitive, and emotional domains. For example, psychosis is inherently characterized by a lack of psychological flexibility that continues to perpetuate delusional convictions despite the presence of contradictory evidence (e.g., Colbert, Peters, \& Garety, 2010; So et al., 2012). In general, individuals with a psychotic disorder also experience impairments in executive functioning, which include high-order neurocognitive processes such as cognitive flexibility (e.g., Reichenberg et al., 2009; Riley et al., 2000). Difficulty with cognitive flexibility and other executive functions has a direct effect on real world interpersonal behaviour, and indirect effects on real world work skills and community participation, for individuals with a diagnosis of schizophrenia (Bowie et al., 2008). Finally, individuals with negative symptoms also experience a restriction in their expression of emotion due to the presence of flattened or blunted affect (e.g., Andreasen, 1984a; Foussias \& Remington, 2010). Despite the prevalence of literature on psychological, cognitive, and emotional flexibility in 
psychosis, the extent to which it is related to the larger systemic construct of family flexibility is unknown.

Family flexibility and resilience is an important, albeit relatively understudied, topic in the early psychosis intervention literature. Many studies employ self-report data to gather information about family resilience, however, every family member has his or her own perspective and self-report data can be confounded by the perspective of the family member who completes the forms. Furthermore, self-report data may not capture the complexity of a family's process of adapting to stressful life events in such a way as to inform potential treatment targets for family intervention. The current study is the first of its kind to apply an established behavioural paradigm, through the use of behavioural coding and state space grids, to the exploration of family flexibility and resilience in early episode psychosis.

Family resilience refers to the dimensions or characteristics of a family that allow it to adapt to unexpected changes or crisis events (McCubbin \& McCubbin, 1993; McCubbin, Thompson, \& McCubbin, 1996). Emotional variability (being able to transition in and out of different affective states) may be one of many dimensions that help to foster family resilience. Previous research has found that emotional variability during family interactions is associated with better emotion regulation (Lewis et al., 2006; Lunkenheimer et al., 2012), open communication and ability to resolve family conflict adaptively (van der Giessen et al., 2014), as well as better clinical outcomes (e.g., Granic et al, 2007; Hollenstein et al., 2004). These protective factors are well known to help foster family resilience (Benzies \& Mychasiuk, 2009). The present study will examine emotional variability during real time family interactions. Measures of 
emotional variability include affective flexibility and its counterpart rigidity, as well as diversity of emotional states and unpredictability in the sequence of emotional states. These four measures of emotional variability are derived from state space grids.

\subsubsection{State Space Grids}

A state space grid is a visual depiction of an interaction pattern, and as such, can be used to examine emotional variability during family interactions in real time (Hollenstein, 2013). By experimentally creating a stressful condition for the family (e.g., a difficult family discussion), family members rely on over-learned, automatic response patterns that have developed over time in similar situations. This method provides an experimental and behavioural counterpart to the self-report scales that are typically used to assess family flexibility.

State space grids have been used to examine behavioural flexibility in families with children who have internalizing or externalizing disorders. For example, affective rigidity during parent-child interactions was associated with concurrent levels of internalizing problems in children (Hollenstein et al., 2004) and families with clinically aggressive children exhibited escalating patterns of coercive behaviour and displayed an inability to exit these coercive interactions (Granic et al., 2007). Although family rigidity has been shown to be associated with more psychopathology, it is just as important, if not more so, to note that family flexibility is associated with positive clinical outcomes. For example, family flexibility during parent-child interactions was associated with a successful treatment response to cognitive-behavioural therapy (Granic et al., 2007). In other words, not getting "stuck" in one behavioural pattern, and being able to break a 
negative interaction pattern, is related to better treatment response. This behavioural phenomenon underlines the critical component of family resiliency.

\subsubsection{The Current Study}

This is the first study to apply state space grid analysis in families with an individual with early episode psychosis. This study aims to identify how family flexibility manifests behaviourally in families with a loved one with early episode psychosis, and to understand how families "bounce back" from stress.

There were four primary hypotheses for this study. Given the symptoms of psychosis, including deficits in cognitive flexibility, flattened affect, and disorganized behaviour and thought processes, I hypothesized that families with an individual with early episode psychosis would be (1) less flexible, (2) more rigid, (3) less diverse in their expression of affect, and (4) more unpredictable in the sequence of their interactions compared to healthy control families.

\subsection{Methods}

\subsubsection{Participants}

Individuals with early episode psychosis and their primary caregiver were recruited to participate in this study. Participants were referred from the Heads Up Early Psychosis Intervention Service at Hotel Dieu Hospital in Kingston, Ontario. Exclusion criteria were substance abuse within the past 3 weeks, a diagnosis of pervasive developmental disability, any medical condition that would interfere with testing such as neurological illness or event (e.g., traumatic brain injury, stroke, seizure), or uncorrectable visual or hearing impairments. 
We recruited a healthy control population via the Queen's University

Developmental Database and Kijiji ads for family dyads with an adolescent/young adult that was age and gender matched. Exclusion criteria for the healthy control group included substance abuse within the past 3 weeks, diagnosis of a psychiatric illness, pervasive developmental disability, any medical condition that would interfere with testing such as neurological illness or event (i.e., traumatic brain injury, stroke, seizure), or uncorrectable visual or hearing impairments.

A total of 13 family dyads (i.e., caregiver and youth/young adult) participated in the study. Three healthy control families were excluded from the study for meeting DSMIV criteria for an Axis I disorder. In total, data were analyzed for five families with an individual with psychosis and five healthy control families that were age and gender matched to the adolescents/ young adults with early psychosis. From here on in, for ease of reading, the adolescent or young adult will be referred to as the "youth" and the parent or guardian will be referred to as the "caregiver" across both groups.

3.2.1.1 Families with an individual with psychosis. Of the five families with an individual with psychosis, all of the caregivers were female and all of the youth were male. All caregivers reported engaging in caregiving activities (e.g., helping with groceries, cleaning, or scheduling appointments). Two caregivers were married, two were separated/divorced and one was in a common law relationship. Three of the caregivers were university educated and the other two were college educated and three of the five caregivers were employed at the time of data collection. In regards to the youth, three of the youth were elementary school educated, one was college educated, and one did not want to report education status. Two of the five youth were employed and three of the 
five youth lived at home at the time of data collection. Four of the youth had been hospitalized at some point for psychiatric reasons. Overall, three of the five families had a history of family therapy and one was currently engaged in family therapy.

3.2.1.2 Healthy control families. Of the five healthy control families, three of the caregivers were female and all five of the youth were male. Four of the five healthy control caregivers were married and one was separated/divorced. All were university educated and employed at the time of data collection. In regards to the youth, all of the youth were high school educated and employed. Four of the five youth lived at home with their caregiver. Overall, two of the five families reported a history of family therapy and one family was currently engaged in family therapy at the time of the study. Upon further questioning, the caregiver revealed that the family was receiving support services (e.g., aid for a child with developmental disabilities or emotional support for a separation/divorce) rather than formal family therapy. Table 3.1 includes a summary of the participant demographic information for both the healthy control and psychosis groups. 
Table 3.1.

Descriptive statistics for participant demographic data.

\begin{tabular}{lcccccc}
\hline & \multicolumn{2}{c}{ Psychosis } & \multicolumn{5}{c}{ Healthy Control } \\
& $M$ & $S D$ & Range & $M$ & $S D$ & Range \\
\hline Caregiver & & & & & & \\
Age & 45.60 & 5.73 & $38-52$ & 44.80 & 6.83 & $34-50$ \\
$\begin{array}{l}\text { No. of children } \\
\text { Youth }\end{array}$ & 1.80 & 0.84 & $1-3$ & 3.40 & 1.14 & $2-5$ \\
Age & 22.40 & 7.50 & $17-34$ & 19.60 & 2.19 & $18-22$ \\
$\begin{array}{l}\text { Age of illness } \\
\text { onset }\end{array}$ & 19.80 & 5.63 & $15-29$ & -- & -- & -- \\
$\begin{array}{l}\text { Months untreated } \\
\text { Months in }\end{array}$ & 49.60 & 106.45 & $0-240$ & -- & -- & -- \\
treatment & 33.70 & 30.29 & $5-72$ & -- & -- & -- \\
$\begin{array}{l}\text { Age at first hosp. } \\
\text { No. of hosp. }\end{array}$ & 17.25 & 2.22 & $15-20$ & -- & -- & - \\
$\begin{array}{l}\text { Months since last } \\
\text { hosp. }\end{array}$ & 5.20 & 6.30 & $0-15$ & -- & -- & -- \\
\hline $\begin{array}{l}\text { Note. Hosp. } \\
2.25\end{array}$ & 1.26 & $1-4$ & -- & -- & -- \\
\hline
\end{tabular}

Note. Hosp. $=$ hospitalization

\subsubsection{Measures}

Each family dyad was separated into different rooms for a confidential individual assessment prior to engaging in a family discussion task.

3.2.2.1 Youth assessment. Youth who participated in the study completed a Demographics Questionnaire that included information about age, gender, education, and family history of psychiatric illness. Youth with early episode psychosis also answered questions about their illness such as age of onset, number of hospitalizations, and length of longest hospitalization. All youth completed the FACES-III and Sheehan Disability Scale (described in Chapter 2). The internal consistency for the total score of the FACESIII was excellent with a Cronbach's alpha of 0.91 . However, the internal consistency for the Sheehan Disability scale was poor at 0.34 . This indicated that youth's assessment of their satisfaction and quality of life across occupational/academic, social, and family 
domains was inconsistent, with some youth reporting extremely low satisfaction, and some reporting extremely high satisfaction across these different domains. As such, the three domains of quality of life will be reported independently.

In addition to the family functioning measure (FACES-III) and the quality of life measure (Sheehan Disability Scale), used in Chapter 2, each youth also completed the Issues Checklist (Robin \& Weiss, 1980) and Symptom Checklist (SCL-90; Derogatis, 1977). Youth with early episode psychosis completed a symptom assessment using the Scale for the Assessment of Negative Symptoms (SANS; Andreasen, 1984a) and the Scale for the Assessment of Positive Symptoms (SAPS; Andreasen, 1984b). Healthy control youth completed a psychiatric screen using the Mini International Neuropsychiatric Interview (MINI; Sheehan et al., 1998). These measures are described in detail below.

\subsection{Issues checklist. Youth completed an Issues Checklist that identified} common topics of conflict within the home (e.g., lying, swearing, curfew, drug use). The participant stated whether or not each item on the Issues Checklist had ever caused a fight between them and their caregiver (yes or no), and if they selected yes, then they were asked to rate how upset they felt thinking about the conflict now. The topic for the family discussion task was selected based on an issue that both the youth and caregiver rated as being contentious at home. If there was no consensus among conflict topics between the youth and caregiver, then the item that was rated as most upsetting was selected for the conflict family discussion.

Youth with early episode psychosis completed a second Issues Checklist that was revised to identify topics of conflict that were specific to families who have an individual 
with psychosis (e.g., communication difficulties, withdrawal from others, unusual or strange behaviour). The items for this scale were based on items from standardized symptom measures such as the SANS and SAPS that were revised so as to be written in lay language. For example, the item of social anhedonia related to not initiating and maintaining friendships from the SANS was re-worded as "lack of interest in developing friendships" on the Issues Checklist.

3.2.2.1.2 Symptom checklist. The SCL-90 is a standardized multidimensional 90item self-report symptom inventory that covered various dimensions of psychological distress. Each item was rated on a 5-point scale, ranging from 0 (not at all) to 4 (extremely). The SCL-90 yielded nine subscales: somatization, obsessive-compulsive behaviour, interpersonal sensitivity, depression, anxiety, hostility, phobic anxiety, paranoia, and psychoticism, in addition to a global severity index. The SCL-90 provides a continuous measure of symptom severity and was completed by both groups. The nine subscales of the SCL-90 ranged in reliability from Cronbach's alphas of 0.60 to 0.94 . The two subscales that had Cronbach's alphas below 0.65 were somatization and paranoid ideation. The Cronbach's alpha for the paranoia subscale increased to 0.74 when one of the subscale items was deleted. Therefore, this revised subscale was used in all further analysis. The somatization subscale did not reach adequate reliability and was therefore excluded from analyses.

\subsection{Interview-based symptom assessment for youth with psychosis. Positive} and negative symptoms are hallmark features of schizophrenia-spectrum disorders and were assessed with a structured interview using the SANS (Andreasen, 1984a) and the SAPS (Andreasen, 1984b). Three Doctoral-level clinical psychology graduate students, 
of which one was the primary investigator, conducted the symptom assessment to determine the presence and severity of positive and negative symptoms. Training for the SANS and SAPS consisted of observation of a licensed clinical psychologist conducting the assessment, regular meetings to discuss administration and scoring with the licensed psychologist, practicing the assessment measure on peers, and practicing the assessment on a volunteer patient, until good inter-rater reliability was obtained among the three assessors and the gold standard of the licensed supervising psychologist. The inter-rater reliability among all four raters for the SANS and the SAPS was good, with an intra-class coefficient (ICC) of 0.82 .

The SANS contained 20 items which are designed to rate the presence and severity of negative symptoms, such as (i) Affective Flattening or Blunting; (ii) Alogia (poverty of speech); (iii) Avolition-Apathy (lack of initiation and motivation); (iv) Anhedonia-Asociality (inability to experience pleasure, social and emotional withdrawal); and (v) Attention. The SAPS contains 30 items designed to assess psychopathology caused by positive symptoms such as (i) Hallucinations; (ii) Delusions; (iii) Bizarre Behaviours; and (iv) Formal Thought Disorder. Each domain assessed specific symptoms and provided a global item that represented the perceived level of severity of the symptoms in that domain. In both measures, ratings were made by trained observers based on a standard clinical interview and observed behaviours during the interview. Each item is scored on a 6 -point scale $(0=$ symptom is absent to $5=$ symptom is present in a severe form). A subscale score was calculated for each domain by summing the scores for each item in that domain, including the global rating. The SANS and SAPS were completed following information collected in a 30 to 45 minute 
structured interview. The internal consistency for the SANS was good with a Cronbach's alpha of 0.81 . The SAPS was divided into two distinct constructs. The internal consistency for both positive symptoms and disorganized symptoms of the SAPS were good, with a Cronbach's alpha of 0.80 and 0.77 , respectively.

\subsection{Psychiatric screen for healthy control youth. Three Doctoral-level}

clinical psychology graduate students, of which one was the primary investigator, conducted the Mini International Neuropsychiatric Interview (MINI) to screen for psychiatric disorders. Training for the MINI consisted of observation of a licensed clinical psychologist conducting the assessment, regular meetings to discuss administration and scoring with the licensed psychologist, and practicing the assessment measure on peers. The MINI is a 30-minute structured diagnostic interview that provides verbatim prompts for the interviewer and solicits a yes or no answer from the interviewee. The MINI provided diagnoses according to the DSM-IV and ICD-10 criteria. The MINI modules employed in this study were: Major Depressive Disorder, Dysthymic Disorder, Mania, Panic Disorder, Agoraphobia, Social Phobia, Specific Phobia, Obsessive-Compulsive Disorder, Generalized Anxiety Disorder, Post-Traumatic Stress Disorder, Alcohol Dependence and Abuse, Drug Dependence and Abuse, and Psychotic Disorders. Each module contained a series of yes/no questions that followed diagnostic criteria for each disorder. The interview allowed for the use of follow-up questions to take into account the necessary dimensions of the criteria, such as frequency, severity, and chronological order. Each module began with a screening question and if the answer to that question was negative, the interviewer moved to the next module. If 
the participant endorsed the screening question, then the interview proceeded to the remaining questions in that module.

3.2.2.2 Caregiver assessment. Caregivers completed the same Demographic Questionnaire as the youth. In addition, caregivers completed self-report questionnaires including the Experience of Caregiving Inventory, Family Support Scale, the Brief COPE, and the FACES-III (described in Chapter 2). The Cronbach's alphas for these questionnaires were $0.98,0.60,0.84$, and 0.95 respectively. Since the Family Support Scale did not reach adequate reliability, an item analysis was conducted. This analysis revealed that Cronbach's alpha increased to 0.73 when the "support from co-workers" item was deleted from the Family Support Scale. Therefore, this item was deleted from the Family Support Scale and this revised scale was used in all further analyses. The Sheehan Disability Scale (described in Chapter 2) was completed both as a third-party rating of quality of life for the family member with psychosis and as a self-report measure for the quality of life of the caregiver. The Cronbach's alpha for their report of the youth's quality of life was 0.84 , however the Cronbach's alpha was 0.56 for their own self-reported quality of life, indicating that the caregivers own assessment of their satisfaction across occupational/academic, social, and family domains is inconsistent. As a result, the three domains of quality of life will be reported separately.

3.2.2.2.1 Issues checklist. Caregivers completed the same Issues Checklists as the youth.

3.2.2.2.2 Symptom checklist. The SCL-90 was used to obtain a measure of symptom severity. For caregivers, several subscales of the SCL-90 had items with zero variance, therefore the Cronbach's alphas were largely inconsistent, ranging from 0.04 to 
0.97. The zero variance items indicate that the caregivers in the current sample did not display the symptoms of psychopathology that were measured on several domains of the SCL-90. Subscales with inadequate reliability (i.e., Cronbach's alpha below 0.65), such as the interpersonal sensitivity, paranoia, and psychoticism subscales, were excluded from further analyses. The Cronbach's alpha for the anxiety subscale increased to 0.67 when one of the subscale items was deleted. Therefore, this item was removed and a revised anxiety subscale was used in place of the original.

3.2.2.2.3 Psychiatric screen. All caregivers, from both the psychosis and healthy control groups, completed the MINI to screen for psychiatric disorders as previously described.

Frequency counts for family cohesion (see Table 3.2), flexibility (see Table 3.3), and general family functioning (see Table 3.4) as reported by both the caregiver and the youth are presented below. Table 3.5 displays the means and standard deviations for all of the youth assessment measures and Table 3.6 includes the means and standard deviations for all of the caregiver assessment measures. 
Table 3.2.

Frequency count for the FACES-III cohesion groups.

\begin{tabular}{l|cc|cc}
\hline & \multicolumn{2}{|c|}{ Caregiver } & \multicolumn{2}{c}{ Youth } \\
& Psychosis & Healthy Control & Psychosis & Healthy Control \\
\hline Disengaged & 3 & 1 & 1 & 3 \\
Separated & 1 & 2 & 4 & 2 \\
Connected & 1 & 2 & 0 & 0 \\
Enmeshed & 0 & 0 & 0 & 0 \\
\hline
\end{tabular}

Table 3.3.

Frequency count for the FACES-III adaptability groups.

\begin{tabular}{l|cc|cc}
\hline & \multicolumn{2}{|c|}{ Caregiver } & \multicolumn{2}{c}{ Youth } \\
& Psychosis & Healthy Control & Psychosis & Healthy Control \\
\hline Rigid & 0 & 1 & 0 & 1 \\
Structured & 1 & 1 & 0 & 3 \\
Flexible & 4 & 1 & 3 & 1 \\
Chaotic & 0 & 2 & 2 & 0 \\
\hline
\end{tabular}

Table 3.4.

Frequency count for the FACES-III general family functioning groups.

\begin{tabular}{l|cc|cc}
\hline & \multicolumn{2}{|c|}{ Caregiver } & \multicolumn{2}{c}{ Youth } \\
& Psychosis & Healthy Control & Psychosis & Healthy Control \\
\hline Balanced & 2 & 2 & 3 & 2 \\
Mid-Range & 3 & 2 & 1 & 2 \\
Extreme & 0 & 1 & 1 & 1 \\
\hline
\end{tabular}


Table 3.5.

Descriptive statistics for youth assessment measures.

\begin{tabular}{|c|c|c|c|c|c|c|}
\hline & \multicolumn{2}{|c|}{ Psychosis } & \multicolumn{4}{|c|}{ Healthy Control } \\
\hline & $M$ & $S D$ & Range & $M$ & $S D$ & Range \\
\hline \multicolumn{7}{|c|}{$\begin{array}{l}\text { QOL } \\
\text { Possible range }=0-10 \\
\text { Higher scores indicate greater satisfactic }\end{array}$} \\
\hline Self: Work/school & 1.80 & 2.68 & $0-6$ & 7.00 & 2.55 & $3-10$ \\
\hline Self: Recreational & 2.20 & 0.84 & $1-3$ & 7.00 & 3.08 & $2-10$ \\
\hline Self: Family/home & 3.20 & 2.86 & $0-7$ & 8.00 & 2.00 & $6-10$ \\
\hline \multicolumn{7}{|c|}{$\begin{array}{l}\text { Symptoms } \\
\text { Possible range }=0-4 \\
\text { Higher scores indicate greater symptom severity }\end{array}$} \\
\hline SANS & 1.64 & 0.78 & $0.79-2.48$ & -- & -- & -- \\
\hline SAPS & 0.82 & 0.31 & $0.32-1.15$ & -- & -- & -- \\
\hline \multicolumn{7}{|c|}{$\begin{array}{l}\text { SCL-90 } \\
\text { Possible range }=0-4 \\
\text { Higher scores indicate } g\end{array}$} \\
\hline $\begin{array}{l}\text { Obsessive- } \\
\text { compulsive }\end{array}$ & 18.20 & 25.86 & $2-63$ & 7.80 & 5.72 & $3-14$ \\
\hline $\begin{array}{l}\text { Interpersonal } \\
\text { sensitivity }\end{array}$ & 11.40 & 10.74 & $2-25$ & 5.20 & 5.81 & $0-14$ \\
\hline Depression & 17.00 & 16.54 & $0-37$ & 5.40 & 6.11 & $1-16$ \\
\hline Anxiety & 12.40 & 14.01 & $0-29$ & 3.40 & 5.41 & $0-13$ \\
\hline Hostility & 2.20 & 1.92 & $0-5$ & 6.00 & 3.53 & $1-10$ \\
\hline Phobic anxiety & 4.60 & 7.80 & $0-18$ & 0.60 & 0.89 & $0-2$ \\
\hline Paranoia & 8.20 & 6.22 & $1-14$ & 5.40 & 7.76 & $0-19$ \\
\hline Psychoticism & 8.40 & 7.80 & $1-17$ & 2.00 & 1.87 & $0-5$ \\
\hline Total score & 94.00 & 88.60 & $11-218$ & 47.00 & 42.00 & $16-120$ \\
\hline
\end{tabular}

Note. $\mathrm{QOL}=$ Quality of life. SANS $=$ Scale for the Assessment of Negative Symptoms. SAPS $=$ Scale for the Assessment of Positive Symptoms. SCL-90 = Symptom Checklist 90. 
Table 3.6.

Descriptive statistics for caregiver assessment measures.

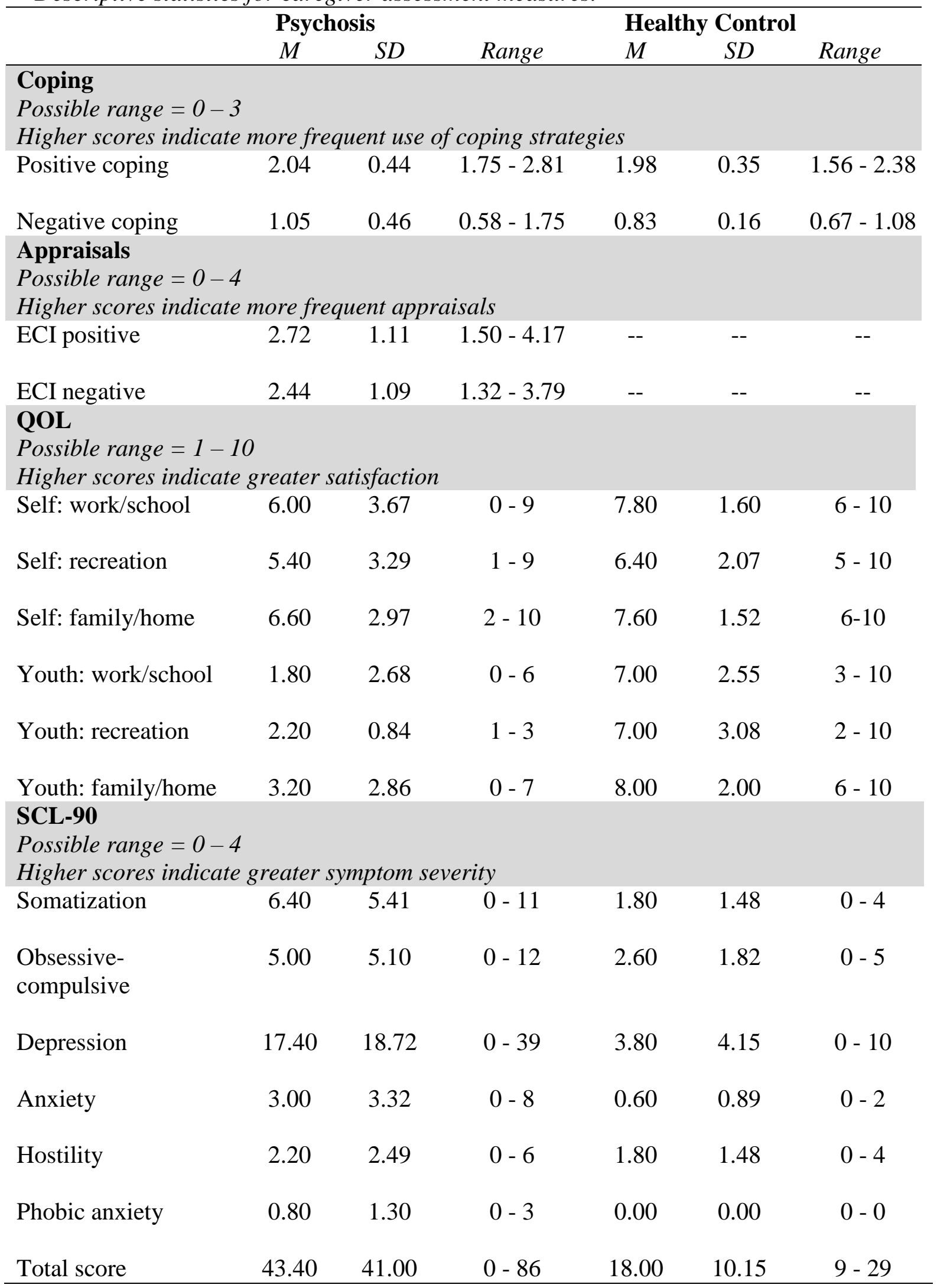

Note. ECI $=$ Experience of caregiving inventory. QOL $=$ Quality of life. SCL-90 = Symptom Checklist 90. 


\subsubsection{Procedure}

The youth and their caregiver were asked to arrive together. At first, they met together with the researchers for general introductions and an overview of the study. After a few minutes, when the family was ready, they were asked to go into separate rooms to obtain consent and begin the individual assessments. Upon completion of the assessments, the youth and caregiver were offered a short break. Afterwards, they came together in a large room to engage in the family interaction task. These interactions were video and audio recorded and coded at a later date. Participation in this study took approximately 2 hours in total. Both family members received $\$ 50$ compensation for their time and travel.

The family interaction task followed an "ABA" design for the healthy control families, and an "ABACA" design for the families with a member with psychosis. Each family dyad was asked to discuss and resolve a fixed order of topics. The first and last topics "A" were always positive (e.g., plan a fun family vacation together given unlimited money). The middle task was a conflict task "B", wherein the dyad was asked to resolve an issue that was selected from the Issues Checklist and rated by both members as being contentious at home. Since the families with a member with psychosis completed two Issues Checklists, their second psychosis-specific issue was the " $\mathrm{C}$ " of the $\mathrm{ABACA}$ design. The ABA component of the design allowed for comparisons between the two groups, whereas the CA allowed for an exploration of family issues specific to the difficulties that families with an individual with psychosis would experience. A positive topic was always selected for the first and final discussion. This design allowed for the examination of behavioural flexibility, as the family dyad was asked to switch between 
contextually diverse affective states between the discussion topics. The dyad was given 5 minutes to discuss each topic and come to a resolution. These interactions were video and audio recorded for coding purposes. The experimenter introduced the topic to the dyad, clarified any questions, and encouraged the dyad to speak to each other naturally (i.e., to each other rather than to the camera).

\subsubsection{Behavioural Coding}

The family interaction tasks were video recorded and coded using a revised 5code version of the Specific Affect (SPAFF) coding system (Gottman et al., 1996). Each code was based on a combination of nonverbal (e.g., facial expressions, gestures, posture) and paralinguistic (e.g., intonation, volume, and speech rate) features, as well as verbal content, in order to capture a gestalt of the affective tone of the youth and caregiver's behaviour. This study employed a revised 5-item version of SPAFF, which consists of the following behavioural categories: (1) Positive Affect, (2) Interest/Curiosity, (3) Neutral, (4) Internalized Negative Affect, and (5) Externalized Negative Affect. Positive Affect was defined as a positive emotional state that included attributes of happiness, affection, caring, laughter, smiling, and enjoyment. Interest/Curiosity was used to capture an emotional state in which the participant communicated an active interest in their partner through non-verbal positive attentive behaviour (e.g., leaning forward, nodding, warm questioning tone of voice), elaboration or clarification seeking, paraphrasing, validation or asking opinion questions. Neutral was used to encompass non-emotional or factual exchanges. Internalizing Negative Affect captured withdrawal behaviours that indicated avoidance or turning inward, such as passivity, sadness, fear, tension, or nervous laughter. Finally, Externalizing Negative Affect comprised of displays of anger, hostility, 
disgust, or domineering behaviours and included attributes such as raised voice, irritation, invalidation, contempt, patronizing, or physical cues of constrained anger.

The SPAFF requires extensive training in integrating multiple channels of information, including non-verbal body and facial behaviour, paralinguistic qualities of varying intensities, and verbal content. Even a relatively simple behaviour such as a laugh could potentially be coded as any of the five categories, including the negative affect categories (e.g., a nervous laugh could be internalizing negative affect or a contemptuous laugh could be externalizing negative affect), as such coders were extensively trained on being able to objectively and reliably categorize ambiguous behaviours.

Coders were two undergraduate-level laboratory staff members who were trained with standard procedures in the SPAFF system. The primary investigator trained the coders. The primary investigator received training from an experimental psychologist who had used the SPAFF system in previous studies. The primary investigator achieved good reliability (Cronbach's alpha above 0.80 ) with the SPAFF system prior to training the coders. Coders were blind to the conditions and hypotheses of the study. Coders' training involved four months of weekly training objectives and coding assignments and practice coding on over 100 videotaped examples from a different study. Furthermore, coders completed two coding "games" as part of training, which involved making flashcards for the different behaviours encompassed within each affective category and writing dialogue scripts that were acted out and coded live by the team. At the end of the four-month training period both coders completed and passed a written and practical coding exam. Once training was complete, both coders coded all of the family 
discussions. Weekly recalibration training was provided to minimize observer drift. The primary investigator compared the coded files from each family in the current study to determine if there were any discrepancies between the raters. All rater discrepancies were resolved in a team-based consensus meeting. Both coders and myself, the primary investigator, attended the consensus meetings. I prepared the files and highlighted the time intervals within each video where there was a rater discrepancy. We then watched the segment of the video together as a team. As I was aware of study hypotheses, I did not make any final decisions on the code; however, when necessary, I helped guide the decision-making process of the coders by reminding them to refer to the SPAFF manual (as opposed to "gut" feelings about the affect of the participants), mediating disagreements, and asking them to each justify their position with concrete examples from the SPAFF manual.

\subsubsection{State Space Grid Measures}

State space grids were created to provide a visual illustration of family interactions in real time. The caregiver's affect is represented along the $x$-axis and the youth's affect is represented along the $y$-axis. Both family members were coded in realtime on five different affect categories: positive affect (e.g., affection, joy) interest/curiosity (e.g., attentive listening, seeking elaboration), neutral (e.g., nonemotional factual exchange), internalized negative affect (e.g., withdrawal, tension, sadness), and externalized negative affect (e.g., anger, hostility, contempt). The codes were determined using a standardized coding protocol, the Specific Affect (SPAFF;

Gottman, McCoy, Coan, \& Collier, 1996) coding system, which captures a gestalt of each family member's affect through a combination of non-verbal behaviour, paralinguistic 
features, and verbal content. A state space grid provides a visual representation of the family interaction (see Figure 1.1, Chapter 1). Each circle (or node) represents an event, for example a node in the very bottom left cell would represent a state where both the youth and caregiver are in a positive affect state. The size of the node represents how long the dyad stayed in that state. Each line represents a transition from one cell to another cell.

Four measures of variability were calculated from the state space grids using the software program GridWare 1.1. (Lamey, Hollenstien, Lewis \& Granic, 2004). The four measures are: transitions (i.e., flexibility), dispersion (i.e., diversity), duration per visit (i.e., rigidity), and entropy (i.e., unpredictability).

(1) Transitions are a measure of dynamic flexibility. All dyads start in one cell of the state space grid and as time progresses they express different affective behaviours that move them to other cells on the grid. Visits are a count of the number of transitions from one cell to another. Transitions are calculated as the number of visits minus one (which is subtracted so that the very first event in the grid is not counted). Higher transition scores indicate greater dynamic flexibility (Hollenstein et al., 2013). (2) Dispersion is a measure of affective diversity. Dispersion is calculated by counting the total number of cells occupied in the grid, wherein a greater number of cells represent a greater range of affective states. The calculation of dispersion also controls for the proportional base rate duration in each cell (in order to not equate a brief visit to one cell with a long visit or repeated visits to that cell). Since each discussion, regardless of content, was five minutes in duration, the calculation of dispersion allows for meaningful comparisons across 
dyads. Higher dispersion scores indicate more diversity of affective states. The formula for calculating dispersion is:

$$
1-\frac{\left(n \Sigma\left(d_{i} / D\right)^{2}\right)-1}{n-1}
$$

where $d_{i}$ is the duration in each cell, $D$ is the total duration, and $n$ is the total number of cells. (3) Duration per visit is a measure of rigidity. Duration per visit (also called average mean duration) is the mean duration spent in each cell and is calculated by taking the total duration in a cell and dividing it by the frequency of visits to that cell. The average of each of these cell durations is then taken in order to obtain an overall rigidity score. This is an estimate of how strongly a dyad gets "stuck" in a particular state. It is the opposite of dynamic flexibility, and as such higher duration per visit scores indicate greater rigidity. (4) Entropy is a measure of unpredictability. The calculation of entropy is based on the formula by Shannon and Weaver (1949):

$$
\Sigma\left(P_{i} * \ln \left(1-P_{i}\right)\right)
$$

where $P_{i}$ is the number of visits to each cell i divided by the total number of visits for the entire trajectory. This calculation determines how many pieces of information are necessary to recreate a sequence event-by-event. For example, a low entropy sequence could be "ABABA", because you only need one piece of information in order to recreate the sequence. If you know when event "A" is, you also know when event "B" will take place. Whereas a high entropy event sequence could be "ABAADCPZKB" because it would require all of the individual pieces of information in order to recreate it (Hollenstein, 2013). Higher entropy scores indicate more unpredictability. 


\subsubsection{Data Analysis}

State space grids were constructed based on the coding procedures from the SPAFF, wherein each behaviour category acted as a cell in the grid. State space grids for each family discussion were created to provide a visual illustration of family interactions in real time. Summary state space grids were also created in order to provide a visual representation of real time family interactions collapsed across groups (psychosis and healthy control) to examine visual trends between the two groups across positive and negative family discussion topics. An attractor analysis was conducted to determine if families spent significantly more time in particular affective states depending on the topic of discussion. As discussed in Chapter 1, attractors are recurring states that characterize a system. In other words, they are a state that family members return to repeatedly, or spend long periods of time in. In the present study, an attractor analysis was used to determine if there were particular regions of the state space grid that a family spent significantly more time in compared to other regions of the grid. Finally, a one-way repeated measures analysis of variance (ANOVA) was conducted for each of the variability measures (flexibility, diversity, rigidity, and unpredictability) to determine the within-subject effects for the psychosis group across the five discussion topics, the within-subjects effect for the healthy control group across the three discussion topics, the between-subjects effect for the psychosis versus healthy control groups in the first three shared discussion topics, and their interactions. Data were bootstrapped for each of the repeated measures ANOVAs. 


\subsection{Results}

\subsubsection{State Space Grids}

State space grids (SSG) were created to provide a visual illustration of family interactions in real time. The SSGs for each family dyad that participated in the current study are included in Appendix B.

The SSGs for one family dyad are presented below along with a narrative that describes the interaction patterns using visual examination. In Figures 3.1 to 3.5 below, five SSGs are presented for one family dyad comprised of a youth with early episode psychosis and his primary caregiver. Each SSG represents one of the five discussions, following an $\mathrm{ABACA}$ design where the first discussion was a positive topic, the second was a normative conflict, the third was a positive topic, the fourth was a psychosisspecific conflict, and the final topic was positive.

Figure 3.1 depicts the youth with psychosis and the primary caregiver engaging in a standardized positive family discussion topic (i.e., plan your next birthday celebration). Upon visual examination of this grid, we can see that both the youth and caregiver remained in positive, interest, and neutral states throughout the discussion. Neither the youth nor the caregiver displayed any internalizing or externalizing negative affect during this discussion. Overall, the youth was predominantly in neutral, while the caregiver was predominantly in neutral and interest. 


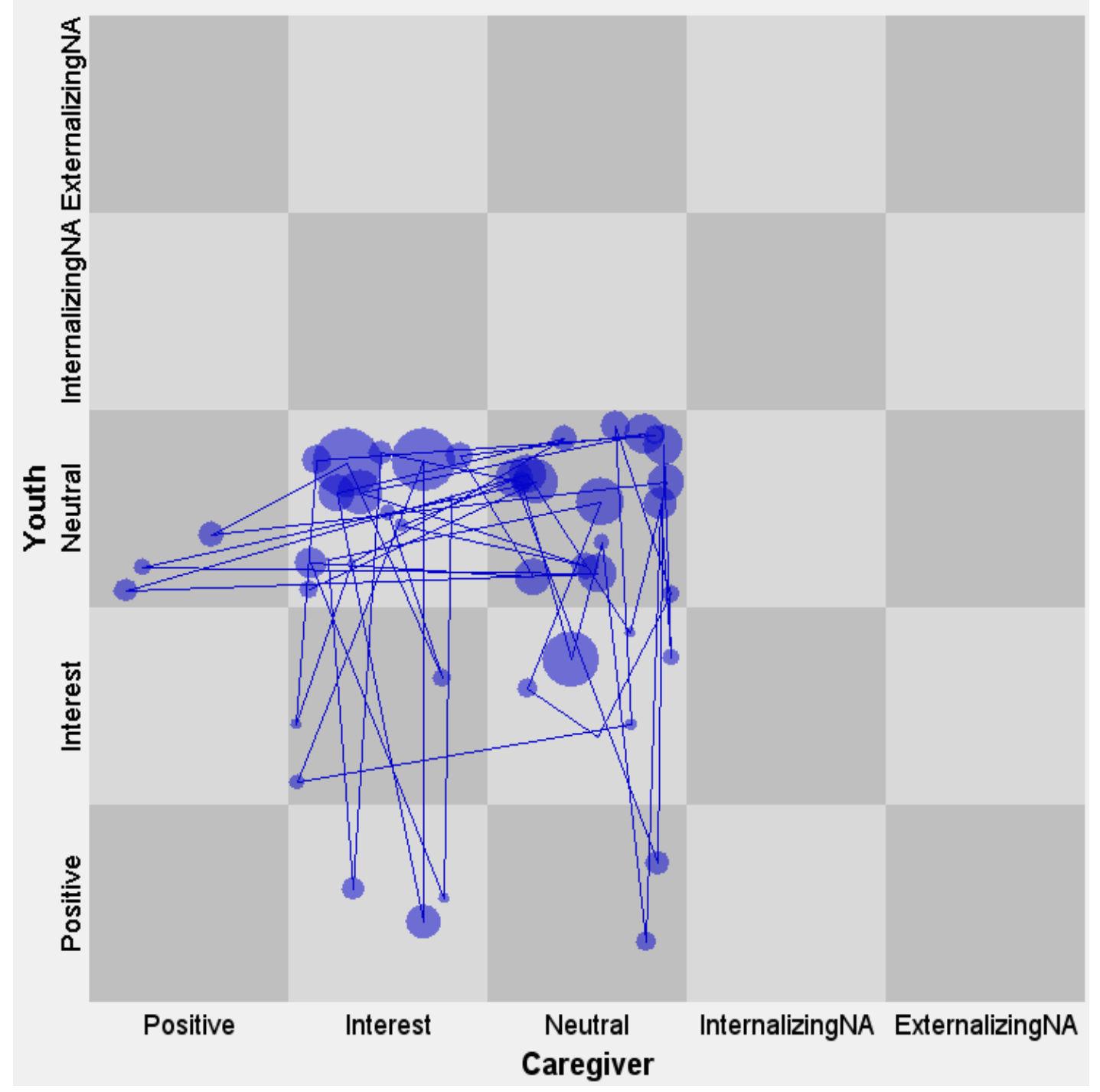

Figure 3.1. State space grid for a positive family discussion between an individual with early episode psychosis and the primary caregiver.

Figure 3.2 below presents the SSG for the same family during a normative conflict discussion that was selected from their dyad's issues checklist (i.e., drinking, smoking, and drug use). In this grid we see a clear restriction in affect, particularly in the youth who remains in neutral for almost the entire discussion (except for two brief transitions to interest and one brief transition to internalizing negative affect). The 
caregiver remains primarily in neutral, with occasional transitions to interest and positive affect and one brief transition to externalizing negative affect.

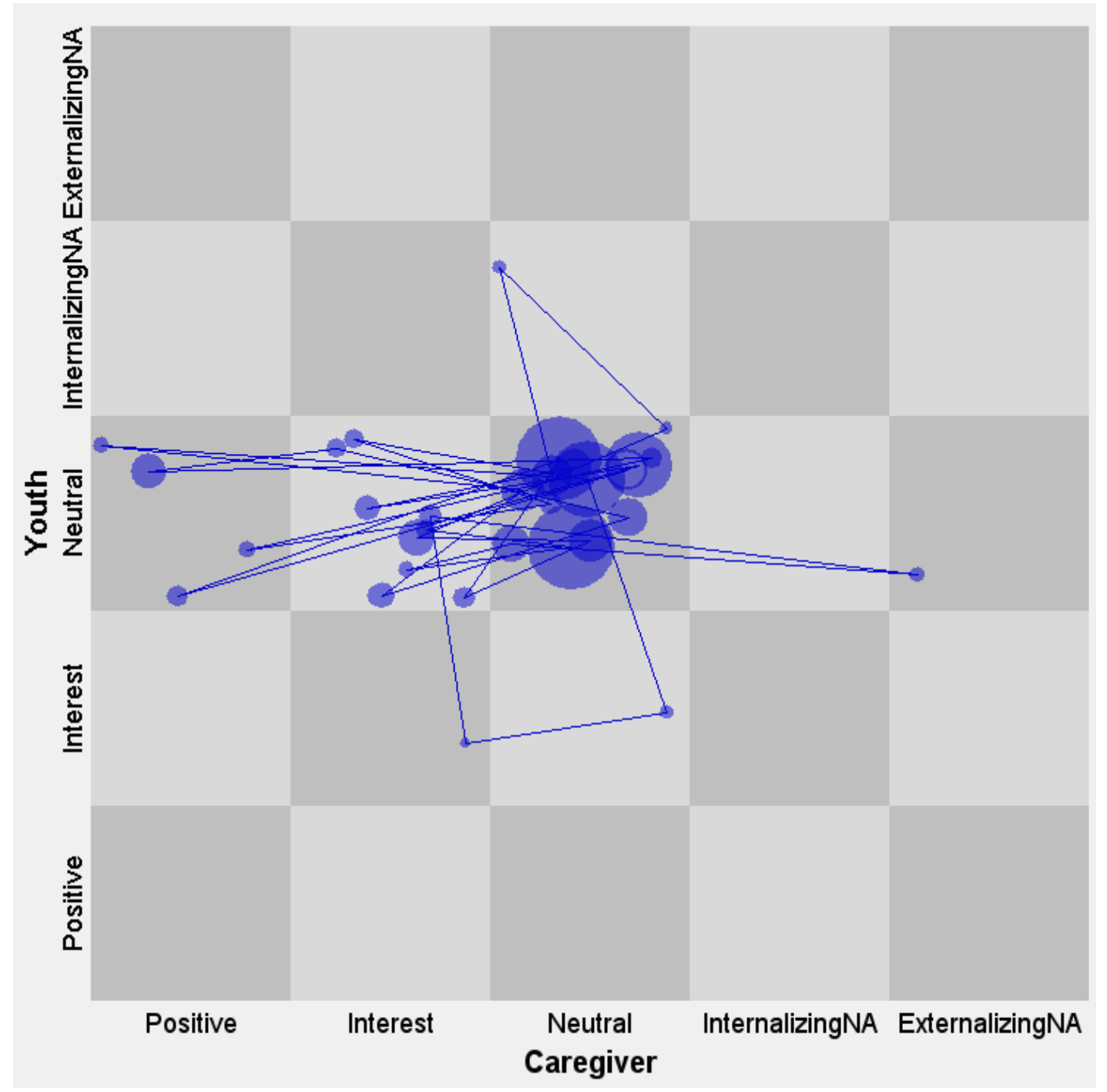

Figure 3.2. State space grid for a family conflict discussion between an individual with early episode psychosis and the primary caregiver.

Figure 3.3 depicts the SSG for the second standardized positive discussion topic (i.e., plan your next family vacation). Similar to the first positive discussion, both the 
caregiver and the youth remain in neutral, interest, and positive affective states with no expression of negative affect. However unlike the first positive discussion, the interaction is primarily neutral with less time spent in positive and interest states. This could suggest some residual tension from the previous conflict discussion.

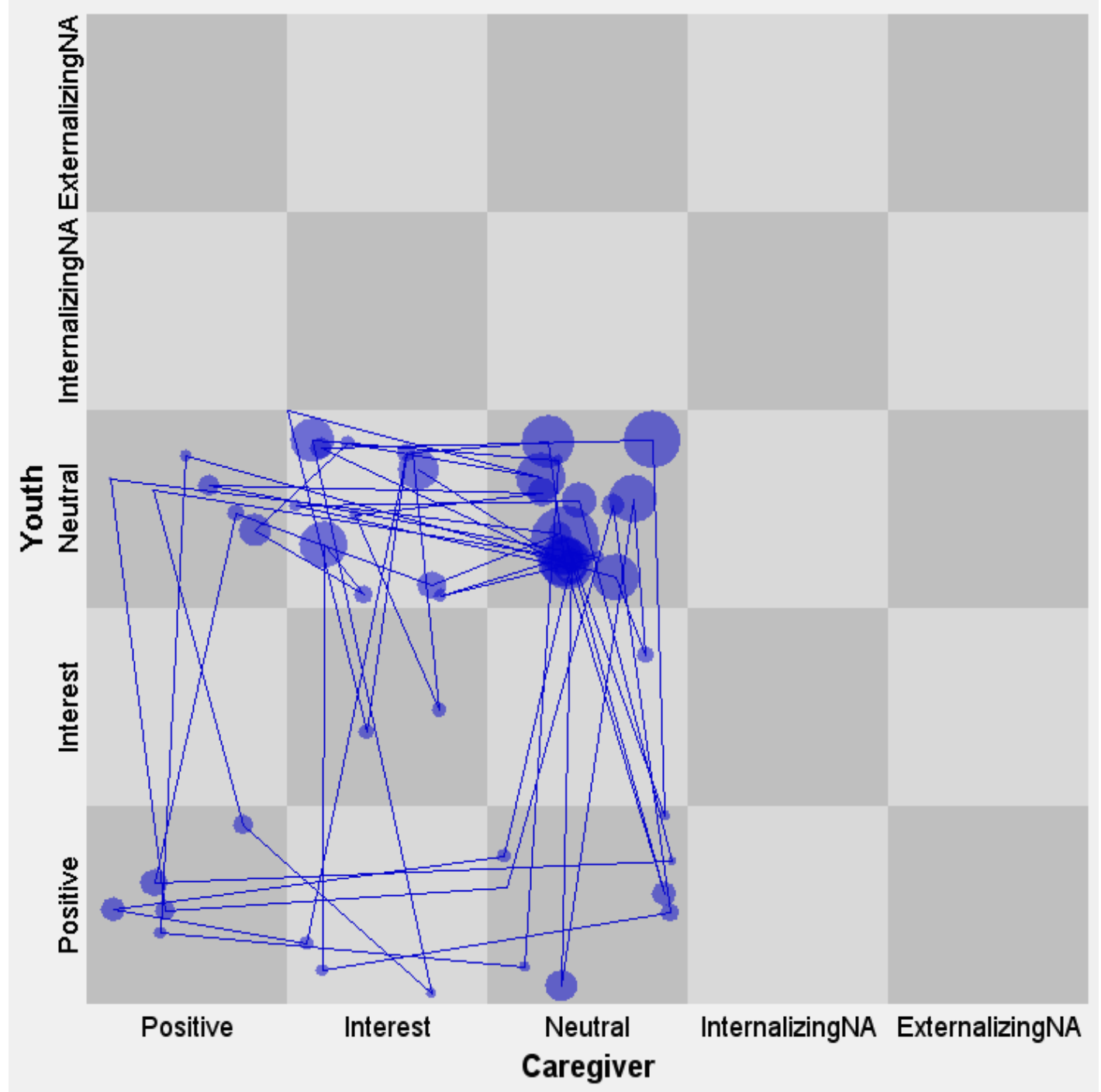

Figure 3.3. State space grid for a second positive family discussion between an individual with early episode psychosis and the primary caregiver. 
Figure 3.4 illustrates the SSG for the psychosis-specific conflict discussion, which was selected from the dyad's issues checklist (i.e., aggressive behaviour). This grid is marked by a restriction of affect in the caregiver who remains primarily in neutral with occasional shifts to interest or internalizing negative affect and one brief transition to positive affect. The youth is also primarily in neutral, however, compared to the normative conflict discussion, the youth displays multiple transitions to externalizing negative affect during this symptom-specific conflict.

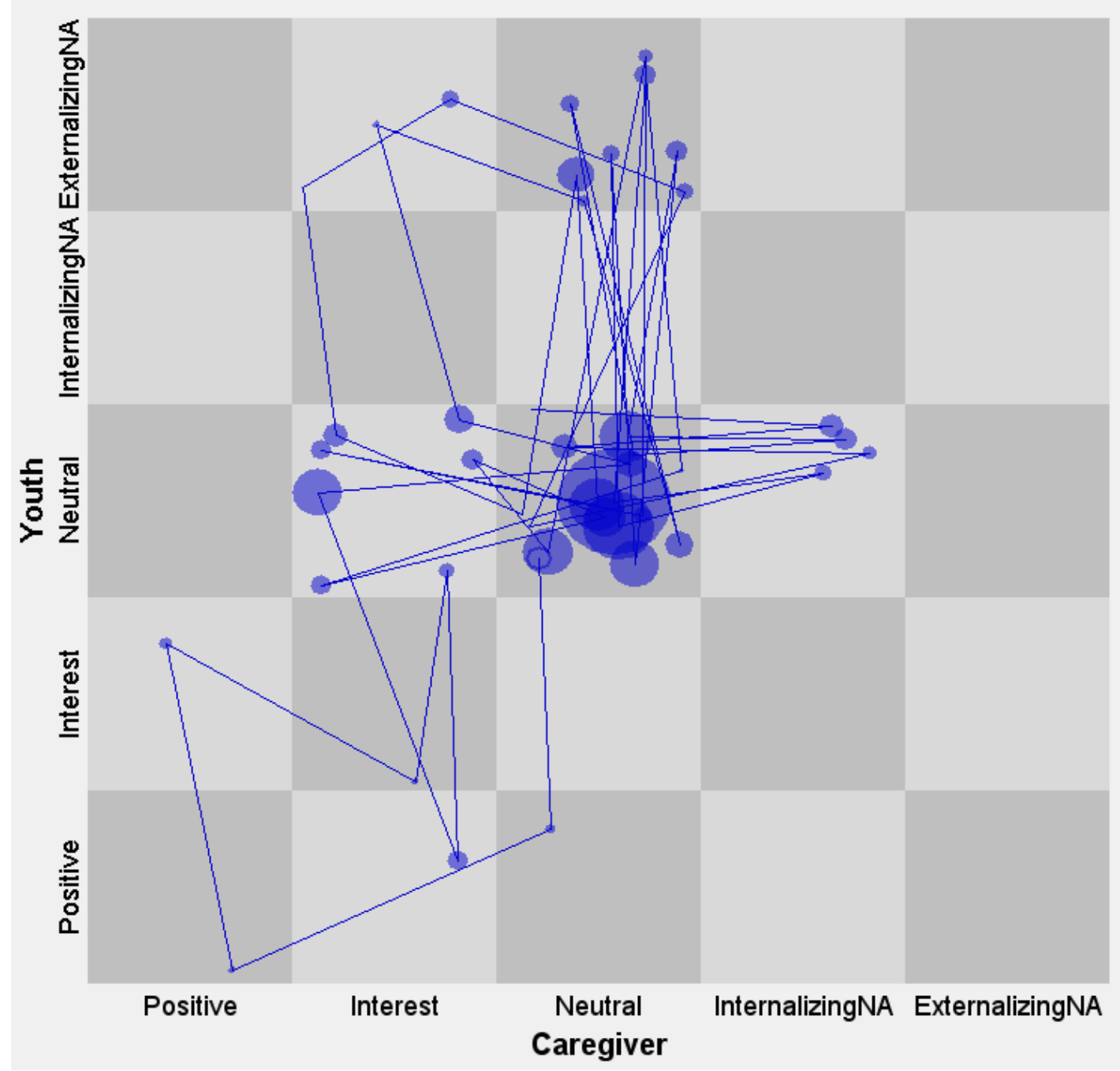

Figure 3.4. State space grid for a psychosis-specific family conflict discussion between an individual with early episode psychosis and the primary caregiver. 
Figure 3.5 presents the fifth and final family discussion. The discussion topic was standardized and positive (i.e., what would you do if you won the lottery). This SSG returns to a similar pattern that was observed in the previous two positive discussions, with affect from both family members in neutral, interest, and positive. However, there is less positive behaviour from both family members, which may be suggestive of residual tension from the previous conflict discussion.

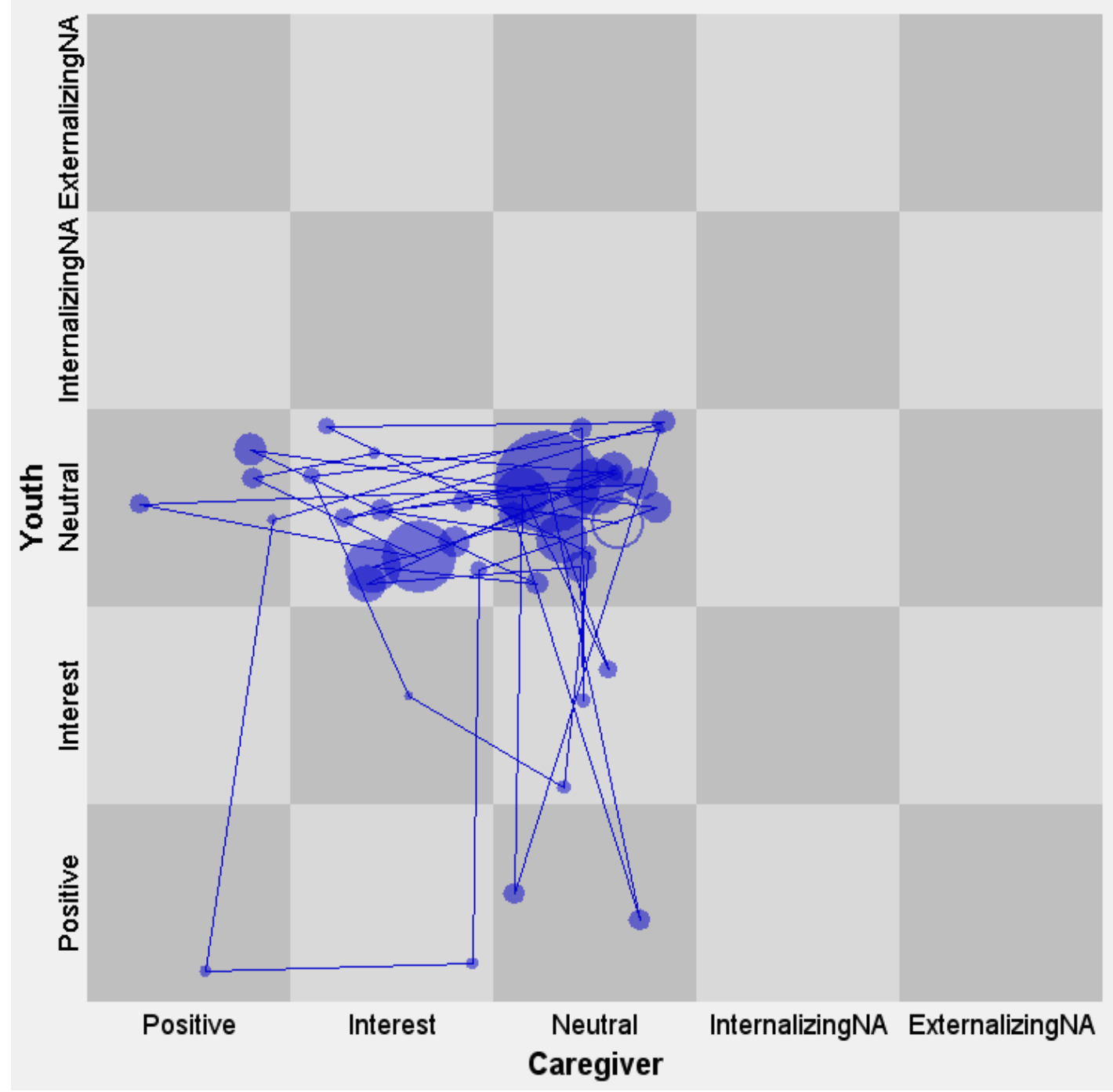

Figure 3.5. State space grid for a third positive family discussion between an individual with early episode psychosis and the primary caregiver. 


\subsubsection{Summary State Space Grids}

In addition to the individual family SSGs, summary SSGs that collapsed across each group (psychosis and healthy control) were created to provide a visual illustration of group differences during the positive and negative discussion topics. Summary grids were collapsed across group such that SSGs for the five families with individuals with early psychosis were combined, and the five healthy control families were combined. The summary SSGs provide an overall summary of the family interactions. Rather than displaying each event and transition between affective states, the summary grids display one single node in each cell that represents the total number of visits to that cell of the grid. The summary state space grids for each group are included below (Figures 3.6 to 3.9). Visual examination of the SSGs demonstrates some minor differences between the two groups across the positive and negative discussion topics. In the first positive family discussion (Figure 3.6), both healthy control families and families with an individual with early psychosis were predominantly characterized by neutral, interest, and positive affect states with some internalizing negative affect on the part of the youth. There was no display of negative affect with the healthy control caregivers, and only infrequent internalizing negative affect with the caregivers of an individual with psychosis. In the normative conflict discussion (Figure 3.7), many similarities between the two groups are observed, where both the youth and the caregivers display more instances of negative affect; however, healthy control families appear to maintain more positive affect during the conflict discussion. In the second positive family discussion (Figure 3.8), both groups display a similar number of neutral, interest, and positive affect events, and youth in both groups exhibit some instances of internalizing negative affect during this positive 
discussion topic. Families with an individual with early psychosis differ from the healthy control families in that youth exhibit some externalizing negative affect and caregivers exhibit some internalizing negative affect. For the families with an individual with psychosis, two additional discussions (a non-normative, symptom-specific conflict and a follow-up positive discussion topic) were included (Figure 3.9). Interestingly, these two grids are remarkably similar with the exception of less interest behaviour from the youth and a brief instance of externalizing negative affect from a caregiver in the follow-up positive discussion. The youth appear to maintain externalizing negative affect across both the symptom-specific conflict discussion and the follow-up positive discussion, suggesting some residual externalized negativity following this psychosis-specific conflict discussion. 

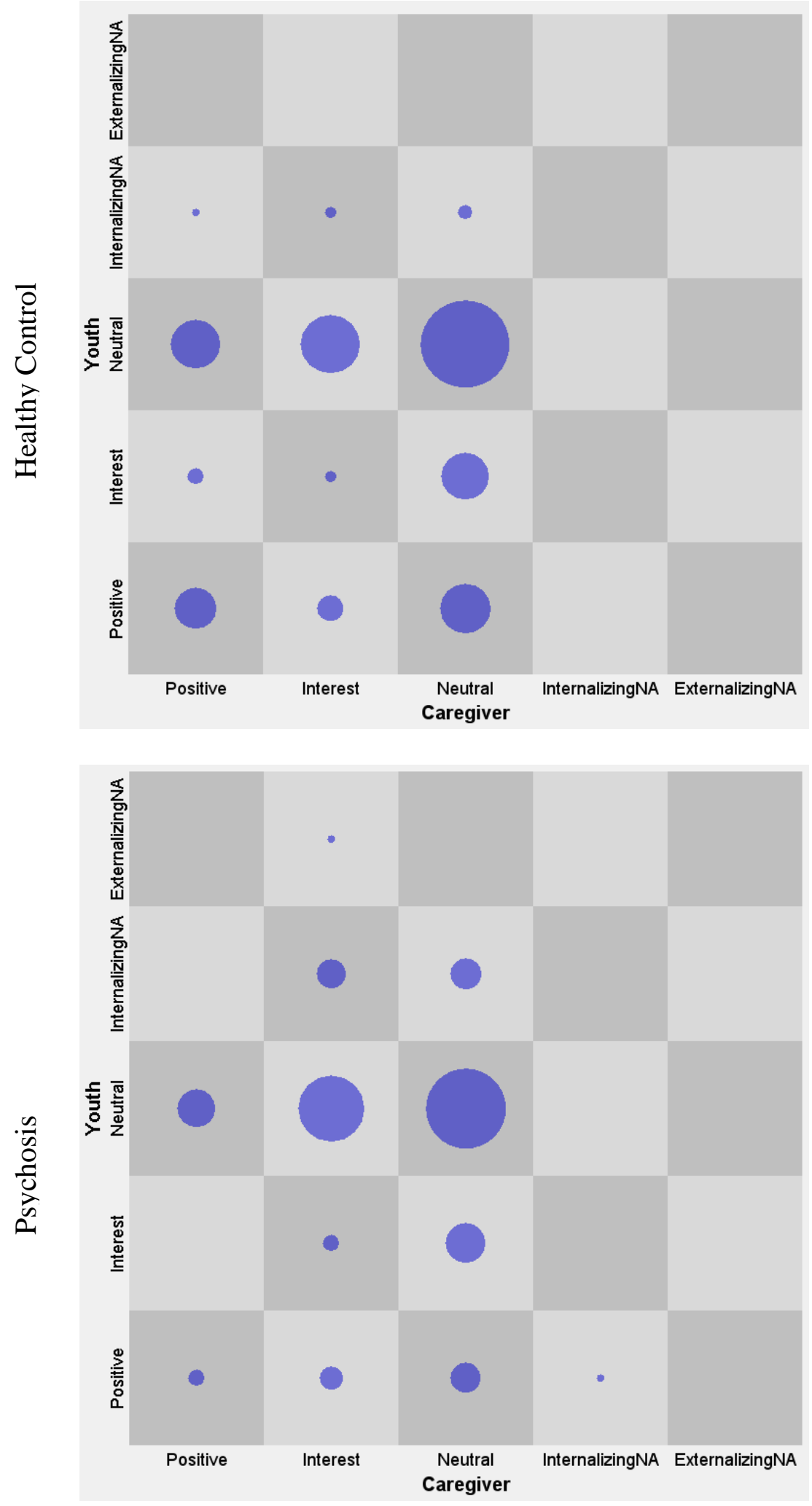

Figure 3.6. Summary state space grids for the first positive family discussion by group. 

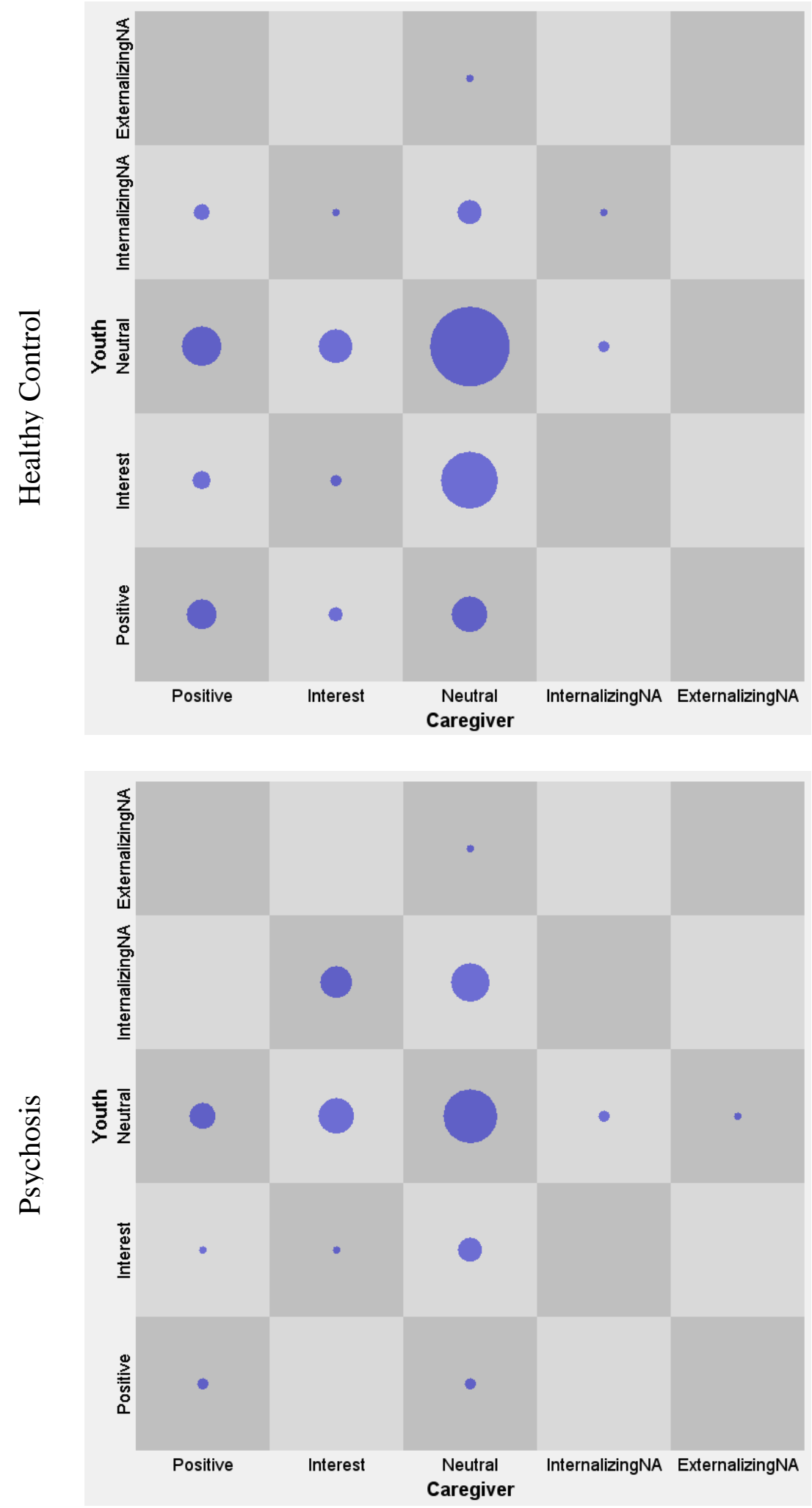

Figure 3.7. Summary state space grids for the normative conflict discussion by group. 

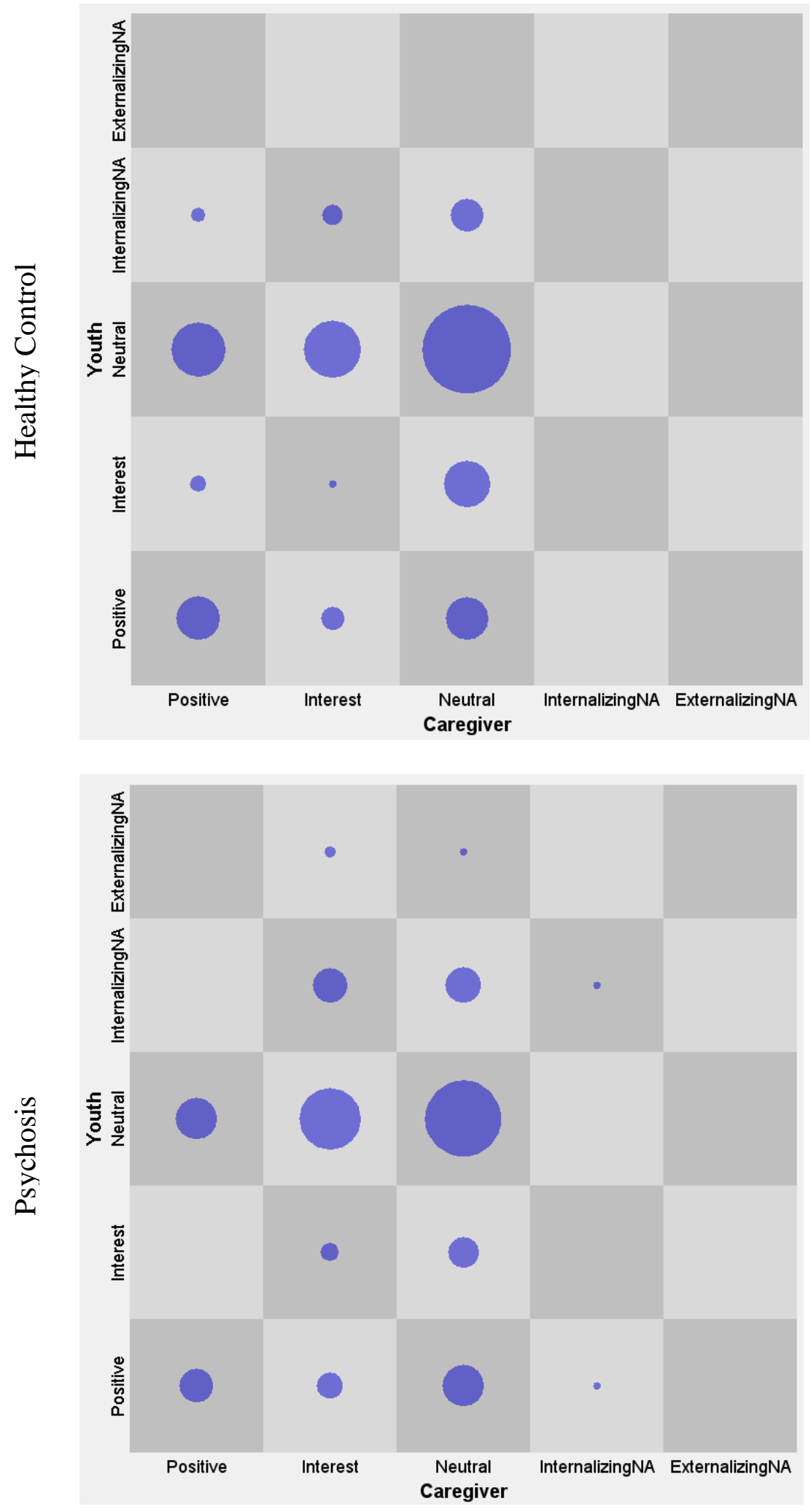

Figure 3.8. Summary state space grids for the second positive discussion topic by group. 

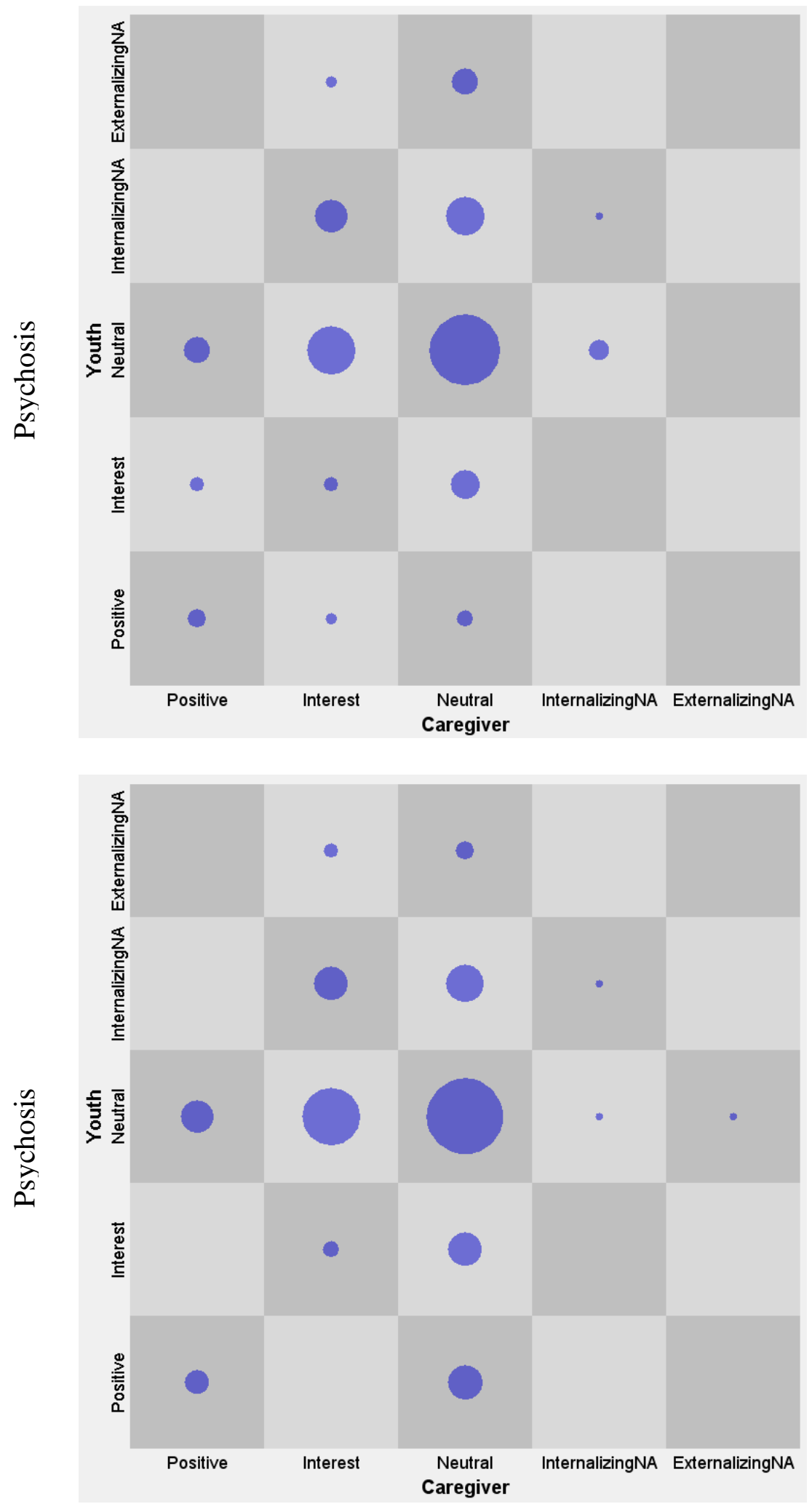

Figure 3.9. Summary state space grids for the psychosis-specific conflict discussion and follow-up positive discussion for the families with an individual with early psychosis. 


\subsubsection{Attractor Analyses}

As described in Chapter 1, attractors are states that a dyad gets repeatedly drawn to, or "stuck in", over time. An attractor analysis can be conducted to determine empirically derived attractors within each state space grid. The theoretical premise for an attractor analysis is that an attractor will occur more frequently than what is expected by chance, and as such an attractor analysis follows a similar procedure to a chi-square analysis where comparisons are made between observed and expected frequencies. Lewis et al., (1999) described how to calculate attractors using a procedure called the winnowing method. The winnowing method eliminates cells where the dyad spends the least amount of time in an iterative process of elimination so that one is left only with the cells with the highest duration. The null hypothesis is that all cells (or affective states) on the state space grid are equivalently distributed. A heterogeneity score is calculated using the following formula:

$$
\text { Heterogeneity }_{j}=\frac{\sum\left(\text { Observed }_{i}-\text { Expected }_{j}\right)^{2} / \text { Expected }_{j}}{\# \text { of Cells }} \text { Exp }_{j}
$$

Where $i$ is an index of the cell and $j$ is an index of the current iteration. As such, the heterogeneity score is calculated at each iterative step in the winnowing process. The heterogeneity scores decreases towards zero as the number of cells decrease through this process of elimination.

Then, heterogeneity values are examined for a drop in proportion similar to a scree plot. The scree value is either the last value drop to zero in the final iteration or where the difference in proportion is greater than $50 \%$. Given the interest in affective 
states, the Neutral - Neutral cell (where both the caregiver and youth are in neutral) was excluded from all attractor analyses.

Attractors were calculated for each grid and are presented in Table 3.7. Overall there were few differences between the healthy control families and the families with an individual with psychosis, such that most attractors were a combination of positive, interest, and neutral states. Caregivers were generally in an attractor state of interest or neutral regardless of the valence of the discussion. Healthy control youth were typically in neutral or interest states, except for one youth (HC3) who was predominantly positive and one youth (HC2) who was predominantly internalizing negative affect. The youth with early psychosis were almost uniformly neutral, with PSY2 and PSY3 demonstrating some attractor states in internalizing negative affect depending on the discussion topic. An exception to this was PSY1 wherein the youth was uniformly in internalizing negative affect across all discussion topics. It is important to note that this youth's symptom profile was distinct from the others as the youth exhibited severe negative symptoms that were likely captured in the internalizing negative affect code (i.e., withdrawal).

Interestingly, across all dyads, there did not appear to be considerable differences in the attractor states between positive and negative discussion topics, although specific cases demonstrated a clear $\mathrm{ABA}$ response, wherein attractor states varied according to the valence of the discussion topic (see HC5, PSY2, PSY3). 
Table 3.7.

Empirically derived attractors for each state space grid.

\begin{tabular}{|c|c|c|}
\hline Family & Discussion & Attractor State (Caregiver - Youth) \\
\hline \multicolumn{3}{|c|}{ Healthy Control } \\
\hline \multirow[t]{3}{*}{$\mathrm{HC} 1$} & Positive & Neutral - Interest \\
\hline & Normative Conflict & Neutral - Interest \\
\hline & Positive & Interest - Neutral \\
\hline \multirow[t]{3}{*}{$\mathrm{HC} 2$} & Positive & Interest - Neutral \\
\hline & Normative Conflict & Neutral - Internalizing \\
\hline & Positive & Neutral - Internalizing \\
\hline \multirow[t]{3}{*}{$\mathrm{HC} 3$} & Positive & Positive - Positive \\
\hline & Normative Conflict & Positive - Positive \\
\hline & Positive & Interest - Neutral \\
\hline \multirow[t]{3}{*}{$\mathrm{HC} 4$} & Positive & Interest - Neutral \\
\hline & Normative Conflict & Interest - Neutral \\
\hline & Positive & Interest - Neutral \\
\hline \multirow[t]{3}{*}{$\mathrm{HC} 5$} & Positive & Positive - Neutral \\
\hline & Normative Conflict & Neutral - Interest \\
\hline & Positive & Positive - Neutral \\
\hline \multicolumn{3}{|c|}{ Psychosis } \\
\hline \multirow[t]{5}{*}{ PSY1 } & Positive & Neutral - Internalizing \\
\hline & Normative Conflict & Neutral - Internalizing \\
\hline & Positive & Neutral - Internalizing \\
\hline & Psychosis-specific conflict & Neutral - Internalizing \\
\hline & Positive & Neutral - Internalizing \\
\hline \multirow[t]{5}{*}{ PSY2 } & Positive & Interest - Neutral \\
\hline & Normative Conflict & Neutral - Internalizing \\
\hline & Positive & Interest - Neutral \\
\hline & Psychosis-specific conflict & Neutral - Internalizing \\
\hline & Positive & Neutral - Interest \\
\hline \multirow[t]{5}{*}{ PSY3 } & Positive & Interest - Neutral \\
\hline & Normative Conflict & Neutral - Internalizing \\
\hline & Positive & Interest - Neutral \\
\hline & Psychosis-specific conflict & Interest - Neutral \\
\hline & Positive & Interest - Neutral \\
\hline \multirow[t]{5}{*}{ PSY4 } & Positive & Interest - Neutral \\
\hline & Normative Conflict & Interest - Neutral \\
\hline & Positive & Interest - Neutral \\
\hline & Psychosis-specific conflict & Interest - Neutral \\
\hline & Positive & Interest - Neutral \\
\hline \multirow[t]{5}{*}{ PSY5 } & Positive & Interest - Neutral \\
\hline & Normative Conflict & Positive - Positive \\
\hline & Positive & Interest - Neutral \\
\hline & Psychosis-specific conflict & Interest - Neutral \\
\hline & Positive & Interest - Neutral \\
\hline
\end{tabular}




\subsubsection{Repeated Measures Analysis of Variance (ANOVA)}

Given the small sample size, data were not normally distributed. All data were bootstrapped as part of each repeated measures ANOVA. There were no outliers (defined as 3.5 standard deviations above or below the mean). The ANOVAs were conducted for each state space grid variable of interest: dynamic flexibility (i.e., number of transitions between cells), diversity (i.e., dispersion across the cells), rigidity (i.e., duration per visit), and unpredictability (i.e., entropy).

3.4.4.1 Dynamic flexibility. Two repeated measures ANOVAs were conducted on the variable of dynamic flexibility (number of transitions). Please refer to Figure 3.10.

\subsection{Within-subjects effect for psychosis group. A 1x5 repeated measures}

ANOVA was conducted for the families with an individual with psychosis across the five discussion topics to examine a within-subject effect of discussion topic (positive, normative conflict, positive, psychosis-specific conflict, positive) on dynamic flexibility. The within-subjects effect for discussion was not significant for the quadratic effect, $F$ (1, $4)=4.95, p=.090$, partial $\eta^{2}=.55$, but did display a significant order 4 polynomial effect, $F(1,4)=12.60, p=.024$, partial $\eta^{2}=.76$.

\subsection{Within-subjects effect for healthy control group. A 1x3 repeated}

measures ANOVA was conducted across the three discussion topics for healthy control families (positive, normative conflict, positive) on dynamic flexibility. The withinsubjects effect was significant, displaying a quadratic effect, $F(1,4)=17.36, p=.014$, partial $\eta^{2}=.81$

\subsection{Between-subjects effect. A 2x3 repeated measures ANOVA was}

conducted to examine the between-subject effect of group (psychosis, healthy control) 
and the interaction between group and discussion for the three discussions that were shared across groups (positive, normative conflict, positive). The between-subject effect for dynamic flexibility was not statistically significant, $F(1,8)=4.24, p=.074$, partial $\eta^{2}=.35$

3.4.4.1.4 Interaction effect. There was no significant group by discussion interaction effect for dynamic flexibility, $F(2,16)=1.11, p=.352$, partial $\eta^{2}=.12$.

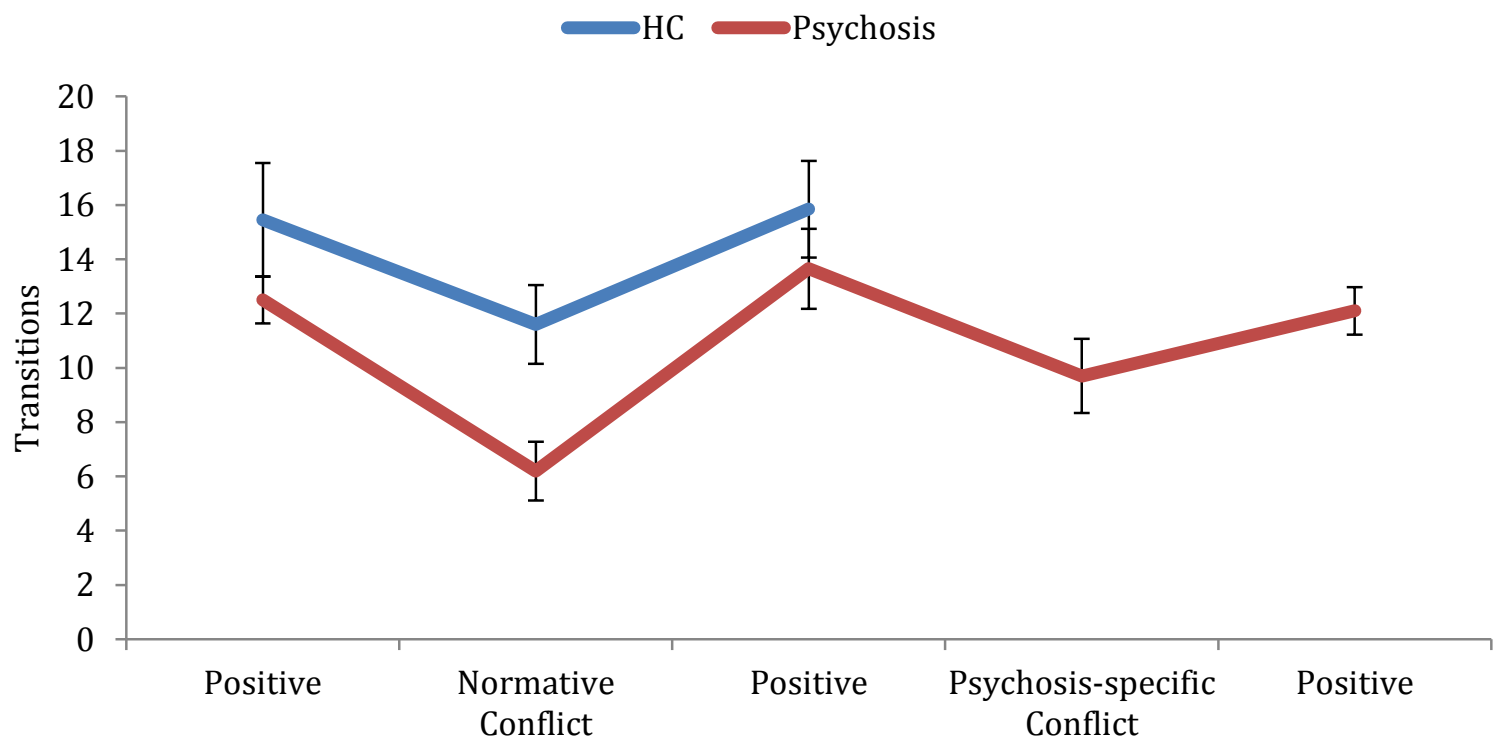

Figure 3.10. Mean number of transitions (i.e., dynamic flexibility) for each discussion topic by group.

3.4.4.2 Diversity. Three repeated measures ANOVAs were conducted on the variable of diversity (dispersion scores). Please refer to Figure 3.11.

3.4.4.2.1 Within-subjects effect for psychosis group. A 1x5 repeated measures ANOVA was conducted for the families with an individual with psychosis across the five discussion topics to examine a within-subject effect of discussion topic (positive, 
normative conflict, positive, psychosis-specific conflict, positive) on diversity. The within-subjects effect of discussion on diversity scores was not statistically significant for a quadratic effect, $F(1,4)=1.51, p=.286$, partial $\eta^{2}=.27$.

\subsection{Within-subjects effect for healthy control group. A 1x3 repeated}

measures ANOVA was conducted across the three discussion topics for healthy control families (positive, normative conflict, positive) on diversity scores. The within-subjects effect was not significant for a quadratic effect $F(1,4)=5.17, p=.085$, partial $\eta^{2}=.56$, but did display a significant linear effect, $F(1,4)=24.91, p=.008$, partial $\eta^{2}=.86$.

3.4.4.2.3 Between-subjects effect. A 2x3 repeated measures ANOVA was conducted to examine the between-subject effect of group (psychosis, healthy control) and the interaction between group and discussion for the three discussions that were shared across groups (positive, normative conflict, positive). The between-subject effect for diversity was not statistically significant, $F(1,8)=0.27, p=.615$, partial $\eta^{2}=.03$.

3.4.4.2.4 Interaction effect. There was no significant group by discussion interaction effect for diversity, $F(2,16)=1.90, p=.182$, partial $\eta^{2}=.19$. 


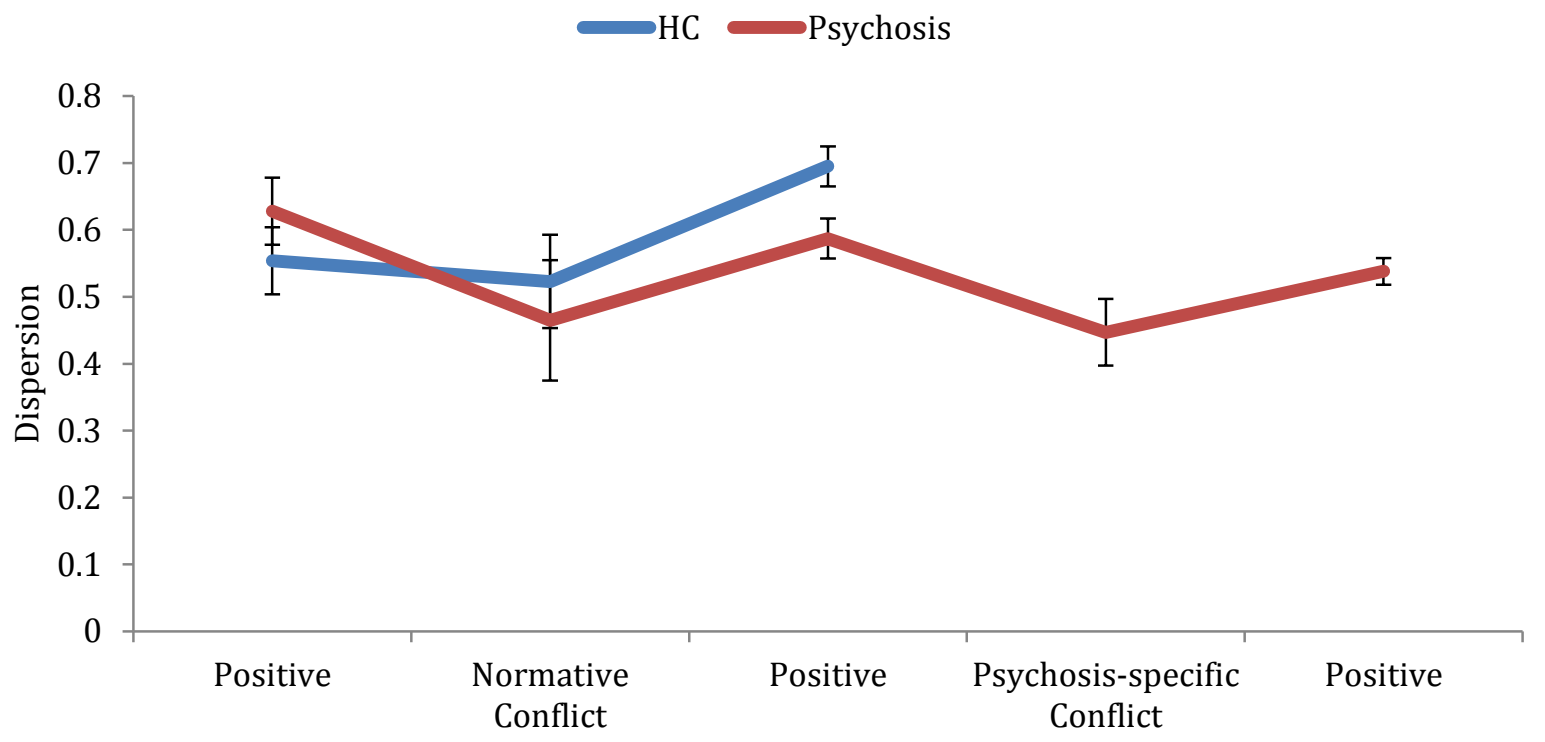

Figure 3.11. Dispersion scores (i.e., diversity of affective states) for each discussion topic by group.

3.4.4.3 Rigidity. Three repeated measures ANOVAs were conducted on the variable of rigidity (duration per visit). Please refer to Figure 3.12.

3.4.4.3.1 Within-subjects effect for psychosis group. A 1x5 repeated measures ANOVA was conducted for the families with an individual with psychosis across the five discussion topics to examine a within-subject effect of discussion topic (positive, normative conflict, positive, psychosis-specific conflict, positive) on rigidity. The withinsubjects effect for discussion was significant, $F(4,16)=3.50, p=.031$, partial $\eta^{2}=.47$, however it did not significantly conform to a quadratic effect.

\subsection{Within-subjects effect for healthy control group. A 1x3 repeated}

measures ANOVA was conducted across the three discussion topics for healthy control families (positive, normative conflict, positive) on rigidity. The within-subjects effect 
displayed a statistically significant quadratic effect, $F(1,4)=13.28, p=.022$, partial $\eta^{2}=.77$

\subsection{Between-subjects effect. A 2x3 repeated measures ANOVA was}

conducted to examine the between-subject effect of group (psychosis, healthy control) and the interaction between group and discussion for the three discussions that were shared across groups (positive, normative conflict, positive). The between-subject effect for rigidity was not statistically significant, $F(1,8)=3.93, p=.083$, partial $\eta^{2}=.33$.

\subsection{Interaction effect. There was no significant group by discussion} interaction effect for rigidity, $F(2,16)=2.33, p=.130$, partial $\eta^{2}=.22$.

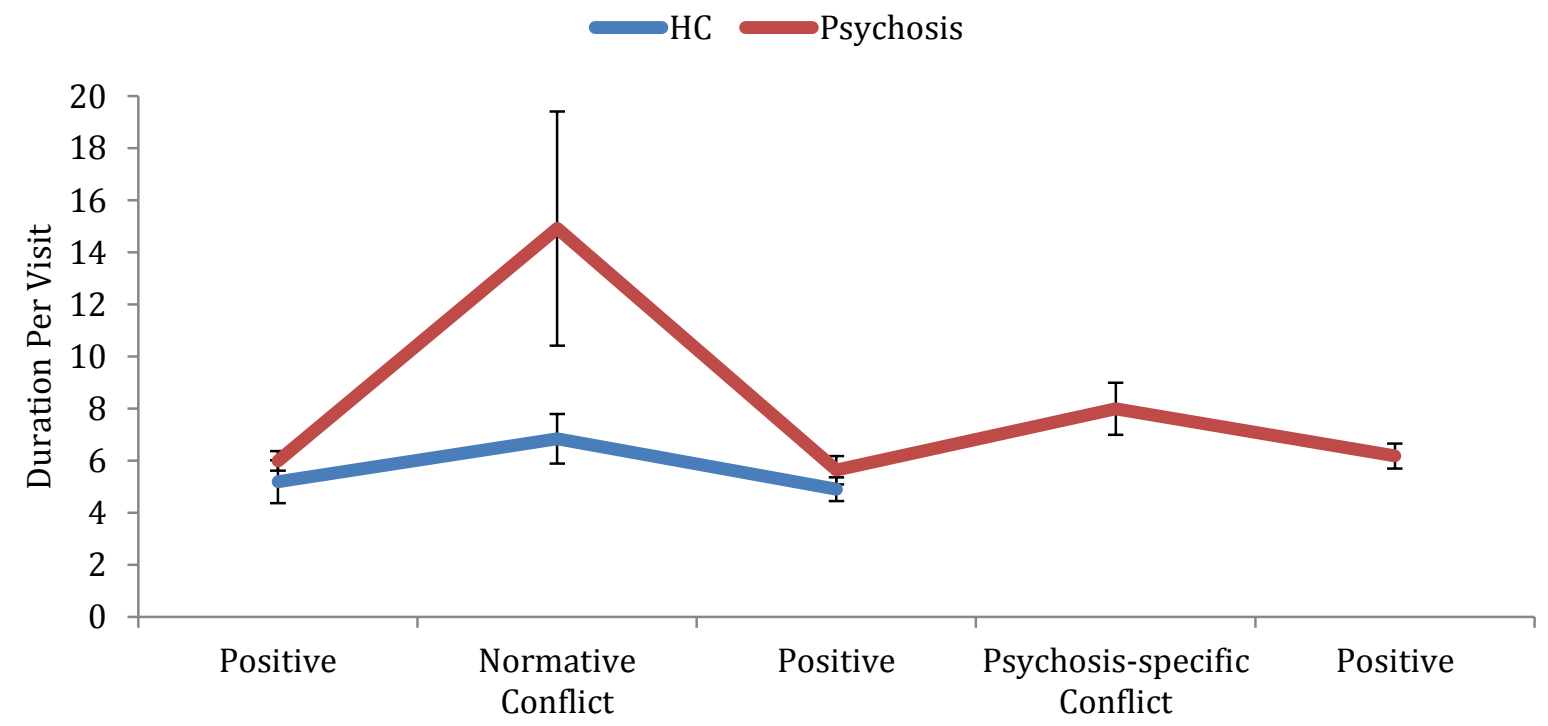

Figure 3.12. Duration of time in seconds per visit (i.e., rigidity) for each discussion topic by group. 
3.4.4.4 Unpredictability. Three repeated measures ANOVAs were conducted on the variable of unpredictability (entropy scores). Please refer to Figure 3.13.

\subsection{Within-subjects effect for psychosis group. A $1 \times 5$ repeated measures}

ANOVA was conducted for the families with an individual with psychosis across the five discussion topics to examine a within-subject effect of discussion topic (positive, normative conflict, positive, psychosis-specific conflict, positive) on unpredictability. The within-subjects quadratic effect for discussion was not significant, $F(1,4)=0.16, p$ $=.707$, partial $\eta^{2}=.04$.

\subsection{Within-subjects effect for healthy control group. A 1x3 repeated} measures ANOVA was conducted across the three discussion topics for healthy control families (positive, normative conflict, positive) on unpredictability. The within-subjects quadratic effect was not statistically significant, $F(1,4)=0.10, p=.771$, partial $\eta^{2}=.02$.

\subsection{Between-subjects effect. A $2 \times 3$ repeated measures ANOVA was}

conducted to examine the between-subject effect of group (psychosis, healthy control) and the interaction between group and discussion for the three discussions that were shared across groups (positive, normative conflict, positive). The between-subject effect for unpredictability was significant, $F(1,8)=5.34, p=.050$, partial $\eta^{2}=.40$, with higher entropy scores for the healthy control group $(M=1.76, S E=0.05)$ than the psychosis group $(M=1.58, S E=0.05)$.

3.4.4.4.4 Interaction effect. There was no significant group by discussion interaction effect for unpredictability, $F(2,16)=0.41, p=.668$, partial $\eta^{2}=.05$. 


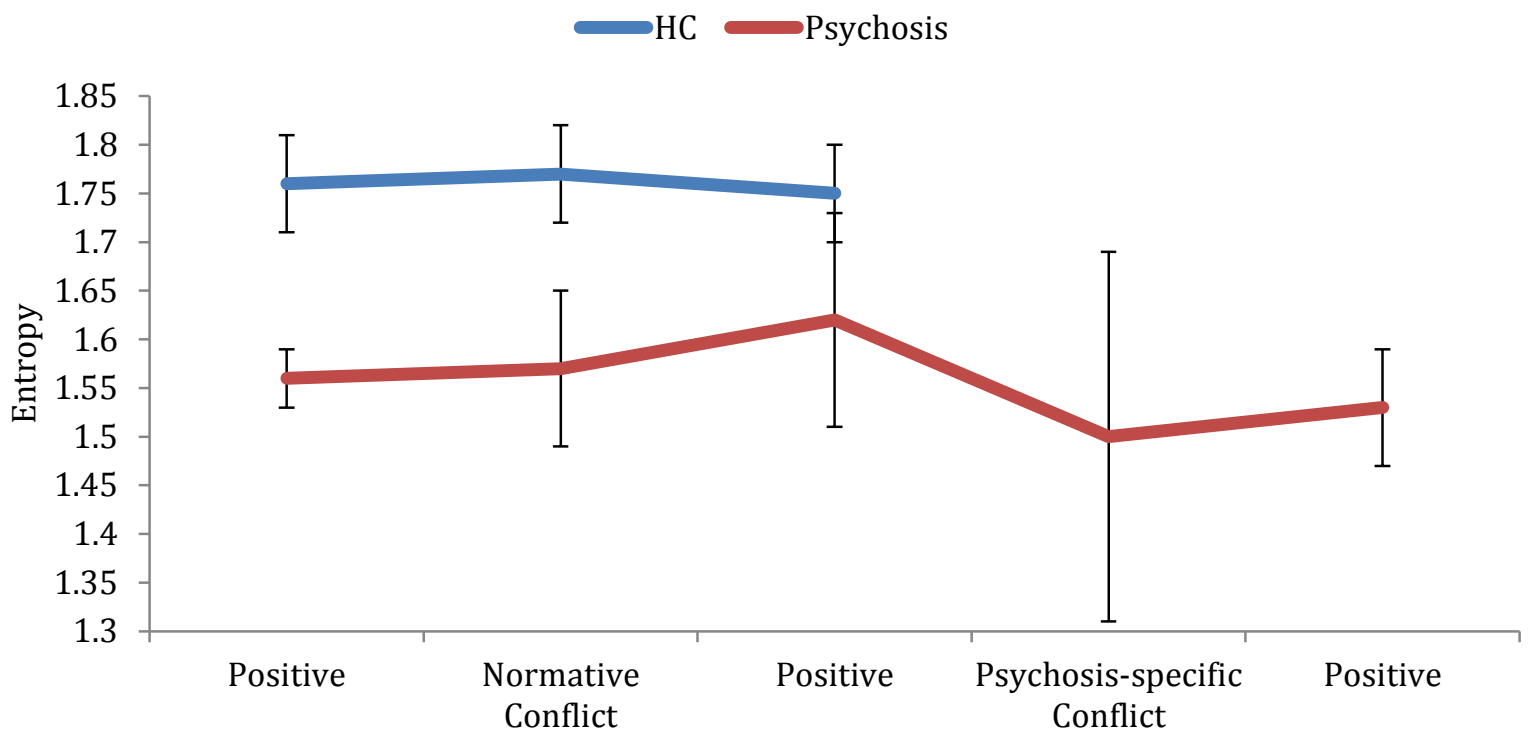

Figure 3.13. Entropy scores (i.e., unpredictability) for each discussion topic by group.

\subsection{Discussion}

The purpose of this family interaction study was two-fold. First, this study aimed to apply state space grids to the field of family research in psychosis in order to better understand family functioning between an individual with early episode psychosis and their primary caregiver. Second, this study examined differences in family interaction patterns between families with an individual with early psychosis and healthy control families across positive and negative family discussion topics.

There is an inherent value in being able to examine the idiosyncratic pattern of emotions and behaviour of each family system on a case-by-case basis. In line with a true systems approach, state space grids allow for the simultaneous evaluation of different individual perspectives within the same family unit; in this case, a youth and their primary family caregiver. Visual examination of each family's state space grid provides detailed information about the ways in which a family adapts to the changing 
circumstances presented by positive and negative family discussion topics. This information is visually illustrated by a change in the pattern of events and transitions across state space grids following an $\mathrm{ABA}$ design that manipulates the emotional context of the conversation (positive - negative - positive). Previous research has shown that family flexibility is lowest during conflict discussions when negative emotions arise, suggesting that dynamic flexibility decreases with the presence of negative emotion (Hollenstein \& Lewis, 2006). This pattern can be observed in the present study (please refer to Appendix B) in grids PSY2, PSY4, and PSY5, wherein affect is restricted during the conflict discussions but rebounds during the positive discussions. Furthermore, one can examine differences in positive and negative affect within the same family unit across different conversational contexts. For example in grids HC4 and PSY4 there is evidence of negative affect only during the conflict discussions, whereas negative affect is present throughout all discussions in grids $\mathrm{HC} 2$ and PSY1. In grids $\mathrm{HC} 3, \mathrm{HC} 5$, and PSY5 positive affect is present across all of the discussions but is less frequent during the conflict discussions. In addition to providing clinically relevant information for each family, state space grids can also be used to examine differences on a group level.

Visual examination of the summary state space grids, in addition to the attractor analysis, demonstrate differences between the psychosis and healthy control family groups across the positive and negative discussion topics. In general, youth with psychosis exhibited a tendency towards more frequent internalizing and externalizing negative affect and fewer expressions of positive affect and interest behaviours compared to the healthy control youth. The youth with psychosis also appeared to maintain externalizing negative affect across both the psychosis-specific conflict discussion and 
the follow-up positive discussion, suggesting some residual anger or hostility when discussing their symptoms with their caregiver. Caregivers across both groups displayed predominantly neutral, interest, or positive affect, with brief instances of internalizing or externalizing behaviour from caregivers of an individual with psychosis, particularly during the conflict discussions and the follow-up positive discussions.

Four measures of emotional variability were derived from the state space grids (i.e., flexibility, diversity, rigidity, and unpredictability). These measures are similar yet distinct estimates of family functioning, such that each evaluates a slightly different facet of family interactions. Dynamic flexibility captures the propensity to shift between affective states. Diversity reflects the repertoire or range of affect displayed during each discussion. Rigidity is a measure of getting "stuck" in particular affective states. Finally, unpredictability represents the degree to which the pattern or sequence of affective states can be anticipated. Analyses revealed that families differed in unpredictability, such that healthy control families were more unpredictable than families with an individual with early psychosis. However, there were no group differences in dynamic flexibility, diversity, or rigidity. These findings suggest that families with an individual with psychosis transition to different emotional states, display a similar repertoire of emotional states, and get stuck in particular emotional states to the same extent as healthy families; however the interactions of families with an individual with psychosis are more predictable in their sequence. This finding was contrary to my hypotheses, as previous research has found increased entropy to be associated with more deviant behaviours (Dishion et al., 2004). However, mean entropy scores for the healthy control group in the present study were considerably lower than those reported in the Dishion and colleagues 
paper. Therefore, although the healthy control dyads in the current study are significantly more unpredictable than the families with an individual with early psychosis, this does not necessarily reflect a more pathological level of unpredictability in the healthy controls. Unpredictability may differ because healthy controls display a mix match of positive and negative states that don't always follow a logical order - this could be a result of a variety of factors (e.g., the use of an inside joke to break the tension during a serious discussion). Healthy control youth tended to exhibit more interest behaviours (compared to neutral behaviours in the youth with psychosis), therefore the conversation may have been more reciprocal, with "back and forth" questioning, that allowed for quick changes in subject that may have resulted in spontaneous changes in affect. Although families with an individual with psychosis displayed the same diversity of affect and flexibly in shifting between affective states, the sequence in which they did so was more orderly and predictable. This may be due, in part, to symptoms of blunted affect and restricted emotional expression that are common in psychosis.

Within each group, the state space grid variability measures exhibited a polynomial effect that confirmed the effectiveness of the ABA design (positive negative - positive). There was an order 4 polynomial effect for the families with an individual with early psychosis for the measure of diversity, as well as a quadratic effect for the healthy control families for flexibility and rigidity. Healthy control families displayed a linear effect for diversity, wherein the range of affect displayed increased over the course of the three discussions perhaps indicating that the families "warmed up" over time. 
This study is the first of its kind. It allows the field to pose new research questions that have not yet been asked in the psychosis literature and begins to answer questions about differences between psychosis and healthy control families in their patterns of interaction, as well as how these families react differently to positive versus conflict discussion topics. Results from this study indicated that families with an individual with early episode psychosis were more predictable in the sequence of their affect during family interactions compared to healthy control families; however, there were no differences between the groups in interpersonal flexibility, rigidity, or diversity of affect. The findings from the present study, although contrary to initial hypotheses, are promising for future research and encouraging for clinical interventions. Although families with an individual with early episode psychosis face unique challenges and stressors (Addington et al., 2003; Barker et al., 2001; Gerson et al., 2009; Jones, 2009; Knock et al., 2011; McCann et al., 2011), the families in the present study displayed adaptive and healthy behaviours in vivo. However, it is important to note that there was a trend in the data for families with an individual with psychosis to be less flexible and more rigid. Although these effects were not statistically significant in the present sample, the effect sizes were quite large. Future research will need to determine if these effects would emerge significant in a larger sample size.

There are several limitations to this study that must be carefully considered when interpreting these results. It is important to keep in mind the potential sampling bias in this study. Recruitment of family members of an individual with early psychosis was unexpectedly challenging, contributing to the small sample size of five family dyads. There are several reasons why recruitment may have been challenging, some of which 
may be idiosyncratic to this project and some of which may have larger methodological implications for family research in psychosis.

Recruitment for this project involved attending family support groups to speak to families directly, meeting with caregivers one-on-one while they were waiting for their family member at a psychiatric appointment, and meeting with clients as they arrived for their appointments. In addition to collecting data from our own clinic, collaborations were attempted at eight clinics across Ontario and Quebec with no success. Reasons for not collaborating included not having the time or resources to support the study. Three of those eight sites offered to make referrals, but not host the research project; however, no referrals from those sites were received during the span of one year. Research ethics were obtained to run the study within the family home to reduce environmental and financial barriers for families who may want to participate; however, no family expressed a preference for this option. When meeting with families, a positive and recovery-oriented approach was employed by making considerable effort to be transparent about all aspects of the study, including debunking myths about research, addressing concerns about 'parent-blaming', and discussing how the purpose of the study was to identify strengths and learn how families support one another through the recovery process. Attempts were made to meet with families in a private room (i.e., not in a waiting room with other people) to allow family members to speak freely and ask questions about the project. Despite all of these efforts, over a period of 15 months, only five families out of the 130 new cases per year in our local program with an individual with early psychosis were willing to participate in the study. Approximately 50 families of these 130 cases were approached in-person by the primary investigator. Therefore, sampling bias is one of the 
primary limitations of this study, as it is possible that I recruited well-functioning families where the youth were coping reasonably well with the illness and the caregivers were actively engaged in their family member's treatment and recovery.

There are several probable explanations for these recruitment challenges. One idiosyncratic issue that may be specific to this study includes primary recruitment at an early intervention clinic where the clinicians making referrals do not frequently engage in research. Gaining trust from both clinicians and families and conveying the importance of research within a clinical context was difficult. However, these recruitment difficulties also pose larger systemic questions about research with families in this population. It is important for researchers and clinicians alike to be sensitive to potential issues related to stigma and concern about 'parent-blaming messages' in this line of research. Many families report feeling stigmatized and report receiving messages of hopelessness about their family member's illness, even from the healthcare professionals involved in their treatment (Gerson et al., 2009).

There is another limitation related to the use of a healthy control population. One in five Canadians experience mental health issues in their lifetime (Smetanin et al., 2011), therefore, a pure "healthy control" family may not be a realistic representation of the average Canadian family. As this was the first study of its kind, I decided to include a control group for precision of experimental design and proof of concept. Future studies should be more inclusive and consider mental health in families as a dimensional rather than categorical variable.

There are many exciting future directions for research that could build upon the present study. With a larger sample, measures of flexibility, diversity, rigidity, and 
unpredictability could be examined in relation to a variety of individual and family variables, such as symptoms, coping strategies, and appraisals to determine differential associations between these variables. In this study, there were no significant differences in flexibility between the healthy control families and families with an individual with psychosis. Mental health research is moving towards a dimensional, rather than categorical, understanding of mental health (Goldberg, 2000; Kessler, 2002; Widiger \& Samuel, 2006). Family flexibility may have little to do with diagnosis and more to do with underlying symptomatology that is inherently dimensional and varies in both clinical and healthy populations. Examining symptoms dimensionally in a larger sample would be more informative for treatment and would help to illuminate mechanisms that may perpetuate dysfunctional family systems (regardless of the presence of a diagnosis).

Furthermore, there was one case in the present study where the youth with psychosis displayed severe negative systems. This family's state space grids were quantitatively and qualitatively different from all others given the static nature of the youth's affect. Examining differences in symptom profiles may also inform differential treatment targets for families with an individual with psychosis who present with severe negative symptoms.

Another interesting direction for future research could include the use of a "perturbation" during the experimental family discussion paradigm. A perturbation involves interrupting the family mid-way through the discussion by knocking on the door and informing them that they have two minutes to resolve their issue. Previous studies have found that a perturbation increases pressure on a family dyad and causes instability within the system, which can trigger a change in their interaction pattern and reveal 
response patterns that may otherwise be unknown. For example, one study found that dyads with children with externalizing disorders only displayed hostility after the perturbation (Granic \& Lamey, 2002). This would be an interesting experimental feature to introduce in future iterations of this design, as it may bring to light dormant interaction states that are only revealed under stress.

Recently, the state space grid literature has expanded beyond child-parent interactions. Researchers have added a third dimension that has been used to examine triadic interactions between a child, mother, and father. One study examined triadic interactions in depressed adolescents and healthy control adolescents, and found that depressed adolescents displayed differences in variability such as a wider range of affect and less predictability in their interactions (Hollenstein, Allen, \& Sheeber, in press). State space grids have also been applied to other fields to look at couples interactions (Burr, Hubler, Larzelere, \& Gardner, 2013), peer interactions (Murphy-Mills, Bruner, Erickson, \& Côté, 2011), as well as teacher-student interactions (Mainhard, Pennings, Wubbels, \& Brekelmans, 2012) and coach-athlete interactions (Erickson, Côté, Hollenstein, \& Deakin, 2011), demonstrating the versatility state space grids as a tool for examining a wide range of interpersonal interactions. Future research in psychosis can also expand upon the present study to include multiple family members such as siblings, who often fall to the wayside and have been termed the "forgotten family members" (Bowman et al., 2014), or to examine other social relationships, such as friendships and romantic relationships. In psychosis, this may be particularly important given the presence of social cognitive deficits and impairments in everyday social functioning (Addington, Saeedi, \& Addington, 2006; Ballon, Kaur, Marks, \& Cadenhead, 2007; 
Bertrand, Sutton, Achim, Malla, \& Lepage, 2007; Bowie et al., 2008). Examining dyadic relationships outside the family would allow for an exploration of the differential effects of social deficits across contextually distinct interpersonal relationships.

Finally, the ability to examine family interactions on both a case and group level presents an exciting avenue for clinical research aimed at testing the effectiveness of family interventions. An experimental paradigm similar to this one could be applied preand post-intervention to determine if families demonstrate increased flexibility following a family-based intervention. This would be an ideal integration of research and practice, as state space grids can provide important research data while giving clinicians meaningful information to work with families one-on-one.

The novel application of a well-established experimental paradigm in this population offers new and important implications for the field. The field of family research in psychosis is limited by its heavy reliance on self-report measures and static instruments of family functioning that are unable to capture the complexity of dynamic family interactions as they unfold and evolve in real time. State space grids provide an innovative way to conceptualize and measure family functioning that can yield meaningful data on both a research and clinical level. Families with an individual with psychosis display more negative affect and less positive affect compared to healthy controls. This pattern is exacerbated in conflict discussions that are focused on resolving issues related to the symptoms of psychosis, which are likely difficult and sensitive topics for both family members. In this study I found that families with an individual with psychosis are significantly more predictable in their interactions compared to healthy controls, but do not differ in flexibility, rigidity, or range of expressive affect. This study 
presents a foundation from which future research can better understand the factors that maintain healthy and adaptive functioning and to inform important treatment targets for family intervention. 


\section{Chapter 4}

\section{General Discussion}

\subsection{Broadening the Conceptualization of Family Functioning}

The early stages of psychosis are often a chaotic, frightening, and stressful period for individuals and their families (Addington et al., 2003; Barker et al., 2001; Gerson et al., 2009; Jones, 2009; Knock et al., 2011; McCann et al., 2011). The present study confirmed that family members of an individual with early episode psychosis report more extreme family difficulties, across both dimensions of cohesion and flexibility. However, even with more self-reported family difficulties, in general families with an individual with early psychosis exhibited healthy patterns of behaviour and affect during in vivo family interactions. The combination of self-report and objective third party data is of utmost importance in the field of family research in psychosis. The integration of selfreport measures and state space grid analysis allows for a fuller and more comprehensive representation of family functioning that can capture the multi-faceted and complex phenomenon of a family system. This project aims to shed new light on the conceptualization and measurement of family functioning and resiliency in psychosis.

\subsection{Theoretical Implications}

The theoretical catalyst for this project materialized in response to several gaps in the early psychosis family literature. First and foremost, existing family studies within this field would often focus either on patient wellbeing or caregiver wellbeing as their outcome variable of interest, without considering the dynamic phenomenon of family wellbeing. Second, the research literature is inordinately fixated on family deficits, and 
does not adequately consider family functioning as a dimensional construct that encapsulates both positive and negative family experiences. Third, family functioning is often assessed with the use of self-report measures, which although useful in some cases, are confounded by the perspective of the family member who completes them and therefore do not provide an objective measure of family dynamics. My dissertation aimed to address these gaps in the literature through two parallel studies: (1) a caregiver selfreport survey study that examined family functioning along dimensions of cohesion and flexibility, and (2) an experimental study that investigated in vivo family interactions and emotional variability of family members across contextually different situations.

In the caregiver survey study, I replicated the stress-coping model of caregiver wellbeing and found that, consistent with previous research, cognitive appraisals and coping strategies significantly contributed to caregiver wellbeing above and beyond illness severity and availability of support systems (Addington et al., 2003; Joyce et al., 2000; Joyce et al., 2003; Szmukler et al., 1996). Negative appraisals about the illness and its consequences, in particular, were strongly associated with lower caregiver quality of life. However, when investigating predictors of family wellbeing in early episode psychosis, positive appraisals emerged as the most prominent predictor of adaptive family functioning. The ability to conjure and maintain positive appraisals, such as a sense of pride and increased closeness to the family member with psychosis, while managing the inherent negative appraisals associated with the stress of an unexpected illness onset appears to foster healthy family functioning in families with an individual with early episode psychosis. This study helps to refine existing theory by providing a next step towards developing a model of family wellbeing in early episode psychosis. 
In the family interaction study, I examined real time changes in affect in families with an individual with early episode psychosis and healthy control families. In general, youth with early episode psychosis displayed more negative affect and less positive affect compared to healthy control youth. Negative affect in the psychosis group appeared to be exacerbated during conflict discussions, particularly when discussing sensitive topics related to psychosis-specific symptoms. This study found that families with an individual with early psychosis were more predictable in their sequence of affective states across positive and negative contexts. Emotional variability as measured by flexibility, rigidity, and diversity of emotional states was similar in families with an individual with early psychosis compared to healthy control families, however, despite not reaching statistical significance the effect sizes in the domains of flexibility and rigidity were large; future work with larger sample sizes will help determine whether these effects reach statistical significance. Emotional variability is an important part of healthy family functioning (e.g., Granic et al., 2007; Granic \& Lamey, 2002; Hollenstein et al., 2004; Hollenstein \& Lewis, 2006; Lewis et al., 2006; Lunkenheimer et al., 2012); however, this is the first study to examine emotional variability in family interactions in psychosis. Future research conducted in larger samples is needed to be able to determine whether emotional variability during family interactions is related to family flexibility and resiliency on a larger scale. It is possible that being able to flexibly shift in and out of different emotional states to engage in family discussions and adaptively resolve conflict may be one of many underlying mechanisms that can promote family resiliency in the face of adverse events.

Though conceptually linked, the theoretical underpinnings of these two studies differ in important ways. The definition and conceptualization of the term flexibility 
differs considerably across these two fields of study. In one, flexibility refers to the shifting of family roles, relationships, and rules in response to environmental changes. In

the other, flexibility refers to emotional variability and being able to transfer in and out of different affective states, the diversity of those affective states, and the predictability of the sequence of those affective states across contextually different environments. Although these distinct constructs and their respective field of study share an interest in how families adapt to change, it is important to keep in mind that they offer unique channels for conceptualization and measurement. The overarching purpose of my dissertation was to take a comprehensive and multi-faceted approach to the study of family functioning in early episode psychosis, and these two studies provided two different orientations with which to achieve that goal. Family functioning is a complex process and we still have a long way to go in understanding how to best support family functioning and resilience in early episode psychosis. Taken together, these two studies expand upon existing theoretical models, challenge our fundamental understanding of the key principles in the field of family functioning in early psychosis by opening new gateways of conceptualization, and provide a platform from which we can better understand this complex, yet undoubtedly important, phenomenon.

\subsection{Clinical Implications}

Several implications for clinical practice emerged from the findings of these studies. Fostering positive appraisals about the illness and the role of caregiving in family members of an individual with psychosis is an important treatment consideration, as positive caregiver appraisals, such a sense of pride and closeness to the affected family member, are critical to healthy family functioning. It is possible that positive appraisals 
are linked to a sense of optimism and hope for recovery. Cognitive appraisals are excellent candidates for standard cognitive behavioural therapy (CBT) techniques. The crux of CBT aims to address maladaptive thoughts and develop more balanced thoughts through a variety of techniques including identifying underlying assumptions and thought patterns, challenging maladaptive thoughts, and testing new thoughts in the real world through carefully constructed behavioural experiments. Similar to the way a therapist might address the negative filter that occurs in depression (where there is a tendency to filter out positive thoughts and ruminate on negative ones), a therapist can also apply these techniques to thoughts related to one's appraisals about the illness, its consequences, and what it means for the caregiver and the family. Caregivers will likely benefit from a strengths-based approach to therapy that validates their difficult experiences while encouraging them to discover positive meanings in these difficult experiences and to re-frame distress in more positive ways. Although youth enrolled in an early psychosis service may have access to a CBT therapist, it is far less common that these services are available to family members. Fostering positive appraisals across all family members may be important for promoting healthy family functioning in the family system. Clinicians may also benefit from the use of multiple modes of measurement. Family resilience is best understood through the integration of subjective and objective data, and patterns of family interactions may be best detected by observing families over time and in different emotional contexts.

Finally, the application of state space grids in family therapy may have the potential to act as a powerful therapeutic aid. Therapists can share the state space grids with families as a tool to identify patterns of interaction and evaluate changes over time. 
Although the labour of behaviour coding may prevent general application of state space grids in clinical practice at this time, clinical treatment trials evaluating the effectiveness of family interventions may benefit from the application state space grids pre- and postintervention as they simultaneously act as a clinical tool and research measure (Granic et al., 2007).

Each family experiences its own unique stressors and challenges as it learns to cope with the unexpected onset of a psychotic illness. However, families also have a great capacity for resiliency in the face of adversity. Approaching the concept of family functioning as a complex, multi-faceted, and ever-evolving phenomenon, both in our clinical practice and in our research measurement, will help us understand how we can best support individuals with psychosis and their families during their process of recovery. 


\section{References}

Addington, J. (2007). The promise of early intervention. Early Intervention in Psychiatry, 1(4), 294-307.

Addington, J., \& Burnett, P. (2004). Working with families in the early stages of psychosis. In J. Gleeson, \& P. McGorry (Eds.), Psychological interventions in early psychosis: A treatment handbook (pp.99-116). Chichester, UK: John Wiley and Sons.

Addington, J., Coldham, E. L., Jones, B., Ko, T., Addington, D. (2003). The first episode of psychosis: the experience of relatives. Acta Psychiatrica Scandinavica, 108, $285-289$.

Addington, J., Collins, A., McCleery, A., \& Addington, D. (2005). The role of family work in early psychosis. Schizophrenia Research, 79(1), 77-83.

Addington, D. E., McKenzie, E., Norman, R., Wang, J., \& Bond, G. R. (2013). Essential evidence-based components of first-episode psychosis services. Psychiatric Services, 64(5), 452-457.

Addington, J., Saeedi, H., \& Addington, D. (2006). Influence of social perception and social knowledge on cognitive and social functioning in early psychosis. The British Journal of Psychiatry, 189(4), 373-378.

Aickin, M., \& Gensler, H. (1996). Adjusting for multiple testing when reporting eseach results: the Bonferroni vs Holm methods. American Journal of Public Health, $86(5), 726-728$.

Allardyce, J., Suppes, T., \& Van Os, J. (2007). Dimensions and the psychosis phenotype. International Journal of Methods in Psychiatric Research, 16(S1), s34-s40. 
American Psychiatric Association. (2013). Diagnostic and statistical manual of mental disorders, (DSM-5). American Psychiatric Association Publication.

Andreasen, N.C. (1984a). Scale for the Assessment of Negative Symptoms (SANS). Iowa City, IA, University of Iowa.

Andreasen, N.C. (1984b). Scale for the Assessment of Positive Symptoms (SAPS). Iowa City, IA, University of Iowa.

Bachmann, S., Bottmer, C., Jacob, S., Kronmuller, K.T., Backenstrass, M., Mundt, C., Renneberg, B., Fiedler, P., \& Schroder, J. (2002). Expressed emotion in relatives of first-episode and chronic patients with schizophrenia and major depressive disorder - a comparison. Psychiatry Research, 112(3), 239-250.

Baldessarini, R. J., Cohen, B. M., \& Teicher, M. H. (1988). Significance of neuroleptic dose and plasma level in the pharmacological treatment of psychoses. Archives of General Psychiatry, 45(1), 79-91.

Ballon, J. S., Kaur, T., Marks, I. I., \& Cadenhead, K. S. (2007). Social functioning in young people at risk for schizophrenia. Psychiatry Research, 151(1), 29-35.

Barker, S., Lavender, T., \& Morant, N. (2001). Client and family narratives on schizophrenia. Journal of Mental Health, 10(2), 199-212.

Barrelet, L., Ferrero, F., Szigethy, L., Giddey, C., \& Pellizzer, G. (1990). Expressed emotion and first-admission schizophrenia. Ninemonth follow-up in a French cultural environment. British Journal of Psychiatry, 156(3), 357-362.

Beiser, M., Erikson, D., Fleming, J. A. E., \& Iacono, W. G. (1993). Establishing the onset of psychotic illness. American Journal of Psychiatry, 150, 1349-1354. 
Benzies, K., \& Mychasiuk, R. (2009). Fostering family resiliency: A review of the key protective factors. Child \& Family Social Work, 14(1), 103-114.

Bertelsen, M., Jeppesen, P., Petersen, L., Thorup, A., Øhlenschlæger, J., Le Quach, P., ... \& Nordentoft, M. (2009). Course of illness in a sample of 265 patients with firstepisode psychosis - five-year follow-up of the Danish OPUS trial. Schizophrenia Research, 107(2), 173-178.

Bertrand, M. C., Sutton, H., Achim, A. M., Malla, A. K., \& Lepage, M. (2007). Social cognitive impairments in first episode psychosis. Schizophrenia Research, 95(1), $124-133$.

Bertolote, J., \& McGorry, P. (2005). Early intervention and recovery for young people with early psychosis: consensus statement. The British Journal of Psychiatry, 187(48), s116-s119.

Birchwood, M., Todd, P., \& Jackson, C. (1998). Early intervention in psychosis. The critical period hypothesis. The British Journal of Psychiatry, 172(33), s53-s59.

Bird, V., Premkumar, P., Kendall, T., Whittington, C., Mitchell, J., \& Kuipers, E. (2010). Early intervention services, cognitive-behavioural therapy and family intervention in early psychosis: systematic review. The British Journal of Psychiatry, 197(5), $350-356$.

Bland, R., \& Darlington, Y. (2002). The nature and sources of hope: Perspectives of family caregivers of people with serious mental illness. Perspectives in Psychiatric Care, 38(2), 61-68. 
Bowie, C. R., Leung, W. W., Reichenberg, A., McClure, M. M., Patterson, T. L., Heaton, R. K., \& Harvey, P. D. (2008). Predicting schizophrenia patients' real-world behavior with specific neuropsychological and functional capacity measures. Biological Psychiatry, 63(5), 505-511.

Bowman, S., Alvarez-Jimenez, M., Wade, D., McGorry, P., \& Howie, L. (2014).

Forgotten family members: the importance of siblings in early psychosis. Early Intervention in Psychiatry, 8(3), 269-275.

Brown, J. I., Fishco, V. V., \& Hanna, G. (1993). Nelson-Denny Reading Test: Manual for scoring and interpretation for forms G \& H. Chicago: Riverside.

Buckley, P. F., Miller, B. J., Lehrer, D. S., \& Castle, D. J. (2009). Psychiatric comorbidities and schizophrenia. Schizophrenia Nulletin, 35(2), 383-402.

Burr, B. K., Hubler, D. S., Larzelere, R. E., \& Gardner, B. C. (2013). Applying the ActorPartner Interdependence Model to Micro-Level Analyses of Marital Partner Affect During Couple Conversations: An Exploratory Investigation. Marriage \& Family Review, 49(4), 284-308.

Butzlaff, R. L., \& Hooley, J. M. (1998). Expressed emotion and psychiatric relapse: a meta-analysis. Archives of General Psychiatry, 55(6), 547-552.

Carver, C. S. (1997). You want to measure coping but your protocol's too long: Consider the Brief COPE. International Journal of Behavioral Medicine, 4(1), 92-100.

Cechnicki, A., Bielanskab, A., Hanuszkiewiczb, I., \& Darenb, A. (2013). The predictive validity of expressed emotions (EE) in schizophrenia. A 20 year prospective study. Journal of Psychiatric Research, 47(2), 208-214. 
Chapman, L. J., Chapman, J. P., Kwapil, T. R., Eckblad, M., \& Zinser, M. C. (1994).

Putatively psychosis-prone subjects 10 years later. Journal of Abnormal Psychology, 103(2), 171-183.

Colbert, S. M., Peters, E. R., \& Garety, P. A. (2010). Delusions and belief flexibility in psychosis. Psychology and Psychotherapy: Theory, Research and Practice, 83(1), $45-57$.

Compton, M. T., Goulding, S. M., Gordon, T. L., Weiss, P. S., \& Kaslow, N. J. (2009). Family-level predictors and correlates of the duration of untreated psychosis in African American first-episode patients. Schizophrenia Research, 115(2), 338-345.

Cumsille, P. E., \& Epstein, N. (1994). Family cohesion, family adaptability, social support, and adolescent depressive symptoms in outpatient clinic families. Journal of Family Psychology, 8(2), 202-214.

Derogatis, L.R. (1977). SCL-90-R, administration, scoring and procedures. Manual for the Revised Version. John Hopkins University School of Medicine.

Dishion, T. J., Nelson, S. E., Winter, C. E., \& Bullock, B. M. (2004). Adolescent friendship as a dynamic system: Entropy and deviance in the etiology and course of male antisocial behavior. Journal of Abnormal Child Psychology, 32(6), 651-663.

Dunst, C. J., Trivette, C. M., \& Hamby, D. W. (1994). Measuring social support in families with young children with disabilities. In C. J. Dunst, C. M. Trivette, \& A. G. Deal (Eds.), Supporting \& strengthening families. Volume 1: Methods, strategies and practices (pp. 152-160). Cambridge, MA: Brookline Books. 
Eaton, W. W., Romanoski, A., Anthony, J. C., \& Nestadt, G. (1991). Screening for psychosis in the general population with a self-report interview. Journal of Nervous and Mental Disease, 179(11), 689-693.

Erickson, K., Côté, J., Hollenstein, T., \& Deakin, J. (2011). Examining coach-athlete interactions using state space grids: An observational analysis in competitive youth sport. Psychology of Sport and Exercise, 12(6), 645-654.

Fogel, A. (1993). Developing through relationships: Origins of communication, self, and culture. Chicago, IL: University of Chicago Press.

Fogel, A., \& Thelen, E. (1987). Development of early expressive and communicative action: Re-interpreting the evidence from a dynamic systems perspective. Developmental Psychology, 23(6), 747-761.

Fortune, D. G., Smith, J. V., \& Garvey, K. (2005). Perceptions of psychosis, coping, appraisals, and psychological distress in the relatives of patients with schizophrenia: An exploration using self-regulation theory. British Journal of Clinical Psychology, 44(3), 319-331.

Foussias, G., \& Remington, G. (2010). Negative symptoms in schizophrenia: avolition and Occam's razor. Schizophrenia Bulletin, 36(2), 359-369.

Gerson, R., Davidson, L., Booty, A., McGlashan, T., Malespina, D., Harold Alan Pincus, M. D., \& Corcoran, C. (2009). Families' experience with seeking treatment for recent-onset psychosis. Psychiatric Services, 60(6), 812-816.

Goldberg, D. (2000). Plato versus Aristotle: categorical and dimensional models for common mental disorders. Comprehensive Psychiatry, 41(2), 8-13. 
Goldberg, D. P., \& Hillier, V. F. (1979). A scaled version of the General Health Questionnaire. Psychological Medicine, 9, 139-145.

González-Blanch, C., Martin-Munoz, V., Pardo-Garcia, G., Martinez-Garcia, O., Alvarez-Jimenez, M., Rodriguez-Sanchez, J. M., Vazquez-Barquero, J. L., \& Crespo-Facorro, B. (2010). Effects of family psychoeducation on expressed emotion and burden of care in first-episode psychosis: a prospective observational study. Spanish Journal of Psychology, 13(1), 389-395.

Gordon, A. Y., \& Salzman, P. (2008). Optimality of the Holm procedure among general step-down multiple testing procedures. Statistics \& Probability Letters, 78(13), $1878-1884$.

Gottman, J. M., McCoy, K., Coan, J., \& Collier, H. (1996). The specific affect coding system (SPAFF) for observing emotional communication in marital and family interaction. Mahwah, NJ: Erlbaum.

Goulding, S. M., Leiner, A. S., Thompson, N. J., Weiss, P. S., Kaslow, N. J., \& Compton, M. T. (2008). Family strengths: a potential determinant of the duration of untreated psychosis among hospitalized African-American first-episode patients. Early Intervention in Psychiatry, 2(3), 162-168.

Granic, I., O’Hara, A., \& Pepler, D. (2007). A dynamic systems analysis of parent-child changes associated with successful "real-world" interventions for aggressive children. Journal of Abnormal Child Psychology, 35, 845-857.

Granic, I., Hollenstein, T., Dishion, T. J., \& Patterson, G. R. (2003). Longitudinal analysis of flexibility and reorganization in early adolescence: a dynamic systems study of family interactions. Developmental Psychology, 39(3), 606-617. 
Granic, I., \& Lamey, A. V. (2002). Combining dynamic systems and multivariate analyses to compare the mother-child interactions of externalizing subtypes. Journal of Abnormal Child Psychology, 30(3), 265-283.

Hanssen, M., Bak, M., Bijl, R., Vollebergh, W., \& van Os, J. (2005). The incidence and outcome of subclinical psychotic experiences in the general population. British Journal of Clinical Psychology, 44(2), 181-191.

Harrison, G., Hopper, K., Craig, T., Laska, E., Siegel, C., Wanderling, J., ... \& Wiersma, D. (2001). Recovery from psychotic illness: a 15-and 25-year international followup study. The British Journal of Psychiatry, 178(6), 506-517.

Heikkila, J., Karlsson, H., Taiminen, T., Lauerma, H., Ilonen, T., Leinonen, K. M., Wallenius, E., Virtanen, H., Heinimaa, M., Koponen, S., Jalo, P., Kaljonen, A., \& Salakangas, R. K. (2002). Expressed emotion is not associated with disorder severity in first-episode mental disorder. Psychiatry Research, 111(2-3), 155-165. Henggeler, S. W., Burr-Harris, A W., Borduin, C. M., \& McCallum, G. (1991). Use of the Family Adaptability and Cohesion Evaluation Scales in child clinical research. Journal of Abnormal Child Psychology, 19(1), 53-63.

Hjärthag, F., Helldin, L., Karilampi, U., \& Norlander, T. (2010). Illness-related components for the family burden of relatives to patients with psychotic illness. Social Psychiatry and Psychiatric Epidemiology, 45(2), 275-283.

Hollenstein, T. (2013). State Space Grids: Depicting Dynamics Across Development. Springer Science \& Business Media.

Hollenstein, T., Allen, N., \& Sheeber, L. (In press). Affective Patterns in Triadic Family Interactions: Associations with Adolescent Depression. 
Hollenstein, T., Granic, I., Stoolmiller, M., \& Snyder, J. (2004). Rigidity in parent-child interactions and the development of externalizing and internalizing behavior in early childhood. Journal of Abnormal Psychology, 32(6), 595-607.

Hollenstein, T., \& Lewis, M. D. (2006). A state space analysis of emotion and flexibility in parent-child interactions. Emotion, 6, 656-662.

Hollenstein, T., Lichtwarck-Aschoff, A., \& Potworowski, G. (2013). A model of socioemotional flexibility at three time scales. Emotion Review, 5(4), 397-405.

Hooley, J. M. (2007). Expressed emotion and relapse of psychopathology. Annual Review of Clinical Psychology, 3, 329-352.

Huber, G., Gross, G., \& Schüttler, R. (1975). A long-term follow-up study of schizophrenia: psychiatric course of illness and prognosis. Acta Psychiatrica Scandinavica, 52(1), 49-57.

Huguelet, P., Favre, S., Binyet, S., González, C., \& Zabala, I. (1995). The use of the expressed emotion index as a predictor of outcome in first admitted schizophrenic patients in a French speaking area of Switzerland. Acta Psychiatrica Scandinavica, 92(6), 447-452.

International Early Psychosis Association Writing Group. (2005). International clinical practice guidelines for early psychosis. The British Journal of Psychiatry, 187(48), s120-s124. 
Jansen, J. E., Lysaker, P. H., Harder, S., Haahr, U. H., Lyse, H. G., Pedersen, M. B., Trauelsen, A. M., \& Simonsen, E. (2014). Positive and negative caregiver experiences in first-episode psychosis: Emotional overinvolvement, wellbeing and metacognition. Psychology and Psychotherapy: Theory, Research and Practice, 87(3), 298-310.

Johnson, S. L., \& Nowak, A. (2002). Dynamical patterns in bipolar depression. Personality and Social Psychology Review, 6(4), 380-387.

Jones, K. (2009). Addressing the needs of carers during early psychosis. Early Intervention in Psychiatry, 3, s22-s26

Jones, P. B., Bebbington, P., Foerster, A., Lewis, S. W., Murray, R. M., Russell, A., Sham, P. C., Toone, B. K., \& Wilkins, S. (1993). Premorbid social underachievement in schizophrenia: results from the Camberwell Collaborative Psychosis Study. British Journal of Psychiatry, 162, 65-71.

Joyce, J., Leese, M., \& Szmukler, G. (2000). The Experience of Caregiving Inventory: further evidence. Society of Psychiatry and Psychiatric Epidemiology, 35, 185-189.

Joyce, J., Leese, M., Kuipers, E., Szmukler, G., Harris, T., \& Staples, E. (2003).

Evaluating a model of caregiving for people with psychosis. Society of Psychiatry and Psychiatric Epidemiology, 38, 189-195.

Keith, S. J., \& Matthews, S. M. (1991). The diagnosis of schizophrenia: a review of onset and duration issues. Schizophrenia Bulletin, 17, 51-67.

Kessler, R. C. (2002). The categorical versus dimensional assessment controversy in the sociology of mental illness. Journal of Health and Social Behavior, 171-188. 
Killackey, E., \& Yung, A. R. (2007). Effectiveness of early intervention in psychosis. Current Opinion in Psychiatry, 20(2), 121-125.

King, S., \& Dixon, M. J. (1995). Expressed emotion, family dynamics and symptom severity in a predictive model of social adjustment for schizophrenic young adults. Schizophrenia research, 14(2), 121-132.

Knock, J., Kline, E., Schiffman, J., Maynard, A., \& Reeves, G. (2011). Burdens and difficulties experienced by caregivers of children and adolescents with schizophrenia-spectrum disorders: a qualitative study. Early Intervention in Psychiatry, 5(4), 349-354.

Koutra, K., Vgontzas, A. N., Lionis, C., \& Triliva, S. (2014a). Family functioning in first-episode psychosis: a systematic review of the literature. Social Psychiatry and Psychiatric Epidemiology, 49(7), 1023-1036.

Koutra, K., Triliva, S., Roumeliotaki, T., Stefanakis, Z., Basta, M., Lionis, C., \& Vgontzas, A. N. (2014b). Family functioning in families of first-episode psychosis patients as compared to chronic mentally ill patients and healthy controls. Psychiatry Research, 219(3), 486-496.

Kraeplin, E. (1919). Dementia praecox and paraphrenia. Edinburgh, UK: Livingstone, $275-276$.

Kuipers, L. (1992). Expressed emotion research in Europe. British Journal of Clinical Psychology, 31(4), 429-443.

Lamey, A., Hollenstein, T., Lewis, M. D., \& Granic, I. (2004). GridWare (Version 1.1). [Computer software]. http://statespacegrids.org 
Larsen, J. T., McGraw, A. P., \& Cacioppo, J. T. (2001). Can people feel happy and sad at the same time?. Journal of Personality and Social Psychology, 81(4), 684-696.

Lavee, Y., McCubbin, H. I., \& Olson, D. H. (1987). The effect of stressful life events and transitions on family functioning and well-being. Journal of Marriage and Family, 49(4), 857-873.

Lazarus, R. S., \& Folkman, S. (1984). Stress appraisal, and coping. Springer, Berlin Heidelberg, New York.

Lee, G., Barrowclough, C., \& Lobban, F. (2014). Positive affect in the family environment protects against relapse in first-episode psychosis. Social Psychiatry and Psychiatric Epidemiology, 49(3), $367-376$.

Lehman, A.F., Kreyenbuhl, J., Buchanan, R.W., Dickerson, F. B., Dixon, L. B., Goldberg, R., Green-Paden, L. D., Tenhula, W. N., Boerescu, D., Tek, C., Sandson, N., \& Steinwahc, D. M. (2004). The schizophrenia patient outcomes research team (PORT). Schizophrenia Bulletin, 30, 193-217.

Lewis, M. D., Granic, I., \& Lamm, C. (2006). Behavioral differences in aggressive children linked with neural mechanisms of emotion regulation. Annals of the New York Academy of Sciences, 1094(1), 164-177.

Lewis, M. D., Lamey, A. V., \& Douglas, L. (1999). A new dynamic systems method for the analysis of early socioemotional development. Developmental Science, 2(4), $457-475$.

Loebel, A. D., Lieberman, J. A., Alvir, J. M., Mayerhoff, D. I., Geisler, S. H., \& Szymanski, S. R. (1992). Duration of psychosis and outcome in first episode schizophrenia. American Journal of Psychiatry 149, 1183-1188. 
Lunkenheimer, E. S., Hollenstein, T., Wang, J., \& Shields, A. M. (2012). Flexibility and attractors in context: Family emotion socialization patterns and children's emotion regulation in late childhood. Nonlinear Dynamics-Psychology and Life Sciences, 16(3), 269-291.

MacCarthy, B., Lesage, A., \& Brewin, C. R. (1989). Needs for care among the relatives of long-term users of day care. Psychological Medicine, 19, 725-736.

Mainhard, M. T., Pennings, H. J., Wubbels, T., \& Brekelmans, M. (2012). Mapping control and affiliation in teacher-student interaction with State Space Grids. Teaching and Teacher Education, 28(7), 1027-1037.

Marshall, M., Lewis, S., Lockwood, A., Drake, R., Jones, P., \& Croudace, T. (2005). Association between duration of untreated psychosis and outcome in cohorts of first-episode patients: a systematic review. Archives of General Psychiatry, 62(9), 975-983.

Martens, L., \& Addington, J. (2001). The psychological well-being of family members of individuals with schizophrenia. Society of Psychiatry and Psychiatric Epidemiology, 36, 128-133.

McCann, T. V., Lubman, D. I., \& Clark, E. (2011). First-time primary caregivers' experience accessing first-episode psychosis services. Early Intervention in Psychiatry, 5, 156-162.

McCrone, P., Singh, S. P., Knapp, M., Smith, J., Clark, M., Shiers, D., \& Tiffin, P. A. (2013). The economic impact of early intervention in psychosis services for children and adolescents. Early Intervention in Psychiatry, 7(4), 368-373. 
McCubbin, M., \& McCubbin, H. (1993). Family coping with health crises: the resiliency model of family stress, adjustment and adaptation. In: Danielson, C., Hamel- Bisell, B., Winstead-Fry, P. (Eds.), Families, Health, and Illness: Perspectives on Coping and Intervention. Mosby, St. Louis, pp. 3-63.

McCubbin, H. I., Thompson, A. I., \& McCubbin, M. A. (1996). Family aAssessment:

Resiliency, Coping, and Adaptation: Inventories for Research and Practice. University of Wisconsin Publishers, Madison.

McGorry, P. D., Edwards, J., Mihalopoulos, C. Harrigan, S. M., \& Jackson, H. J. (1996). EPPIC: an evolving system of early detection and optimal management. Schizophrenia Bulletin, 22(2), 305-326.

McGorry, P. D., Killackey, E., \& Yung, A. (2008). Early intervention in psychosis: concepts, evidence and future directions. World Psychiatry, 7(3), 148-156.

McGorry, P. D., \& Yung, A. R. (2003). Early intervention in psychosis: An overdue reform. Australian and New Zeaaland Journal of Psychiatry, 37(4), 393-398.

McKeown, R. E., Garrison, C. Z., Jackson, K. L., Cuffe, S. P., Addy, C. L., \& Waller, J. L. (1997). Family structure and cohesion, and depressive symptoms in adolescents. Journal of Research on Adolescence, 7(3), 267-281.

McNab, C., Haslam, N., \& Burnett, P. (2007). Expressed emotion, attributions, utility beliefs, and distress in parents of young people with first episode psychosis. Psychiatry Research, 151(1-2), 97-106. 
Meneghelli, A., Alpi, A., Pafumi, N., Patelli, G., Preti, A., \& Cocchi, A. (2011).

Expressed emotion in first-episode schizophrenia and in ultra high-risk patients: results from the Programma 2000 (Milan, Italy). Psychiatry Research, 189(3), 331338.

Meyer, B. (2001). Coping with severe mental illness: Relations of the Brief COPE with symptoms, functioning, and well-being. Journal of Psychopathology and Behavioral Assessment, 23(4), 265-277.

Mihalopoulos, C., Harris, M., Henry, L., Harrigan, S., \& McGorry, P. (2009). Is early intervention in psychosis cost-effective over the long term?. Schizophrenia Bulletin, 35(5), 909-918.

Ministry of Health and Long Term Care. (2011). Early Psychosis Intervention Program Standards. http://www.health.gov.on.ca/english/providers/pub/mental/epi _program_standards.pdf

Minuchin, S. (1974). Families and family therapy. Oxford, UK: Harvard University Press.

Möller-Leimkuhler, A. M. (2005). Burden of relatives and predictors of burden. Baseline results from the Munich 5 year-follow-up study on relatives of first hospitalized patients with schizophrenia or depression. European Archives of Psychiatry and Clinical Neuroscience, 255(4), 223-231.

Mo'tamedi, H., Rezaiemaram, P., Aguilar-Vafaie, M. E., Tavallaie, A., Azimian, M., \& Shemshadi, H. (2014). The relationship between family resiliency factors and caregiver-perceived duration of untreated psychosis in persons with first-episode psychosis. Psychiatry Research, 219(3), 497-505. 
Murphy-Mills, J., Bruner, M. W., Erickson, K., \& Côté, J. (2011). The utility of the state space grid method for studying peer interactions in youth sport. Journal of Applied Sport Psychology, 23(2), 159-174.

Ohaeri, J. U. (2003). The burden of caregiving in families with a mental illness: a review of 2002. Current Opinions in Psychiatry, 16, 457-465.

Olson, D. H. (2000). Circumplex model of marital and family systems. Journal of Family Therapy, 22(2), 144-167.

Olson, D. (2011). FACES IV and the circumplex model: Validation study. Journal of Marital and Family Therapy, 37(1), 64-80.

Olson, D. H., Portner, J., \& Lavee, Y. (1985). FACES III Manual. Family Social Science, University of Minnesota, St. Paul, MN.

Olson, D. H., Sprenkle, D. H., \& Russell, C. S. (1979). Circumplex model of marital and family systems I: Cohesion and adaptability dimensions, family types, and clinical applications. Family Process, 18(1), 3-28.

Onwumere, J., Bebbington, P., \& Kuipers, E. (2011). Family interventions in early psychosis: specificity and effectiveness. Epidemiology and Psychiatric Sciences, 20(2), 113-119.

Patterson, P., Birchwood, M., \& Cochrane, R. (2005). Expressed emotions as an adaptation to loss: prospective study in first-episode psychosis. British Journal of Psychiatry, 187(48), s59-s64.

Peters, E. R., Joseph, S. A., \& Garety, P. A. (1999). Measurement of delusional ideation in the normal population: introducing the PDI (Peters et al. Delusions Inventory). Schizophrenia Bulletin, 25(3), 553-576. 
Poulton, R., Caspi, A., Moffitt, T. E., Cannon, M., Murray, R., \& Harrington, H. (2000). Children's self-reported psychotic symptoms and adult schizophreniform disorder: a 15-year longitudinal study. Archives of General Psychiatry, 57(11), 1053-1058.

Prigogine, I., \& Stengers, I. (1984). Order out of chaos. New York: Bantam.

Pruessner, M., Iyer, S. N., Faridi, K., Joober, R., Malla, A. K. (2011). Stress and protective factors in individuals at ultra-high risk for psychosis, first episode psychosis and healthy control. Schizophrenia Research, 129(1), 29-35.

Raune, D., Kuipers, E., \& Bebbington, P. E. (2004). Expressed emotion at first-episode psychosis: investigating a carer appraisal model. British Journal of Psychiatry, 184(4), 321-326.

Reichenberg, A., Harvey, P. D., Bowie, C. R., Mojtabai, R., Rabinowitz, J., Heaton, R. K., \& Bromet, E. (2009). Neuropsychological function and dysfunction in schizophrenia and psychotic affective disorders. Schizophrenia Bulletin, 35(5), $1022-1029$.

Riley, E. M., McGovern, D., Mockler, D., Doku, V. C., ÓCeallaigh, S., Fannon, D. G., ... \& Sharma, T. (2000). Neuropsychological functioning in first-episode psychosisevidence of specific deficits. Schizophrenia Research, 43(1), 47-55.

Rinaldi, M., Mcneil, K., Firn, M., Koletsi, M., Perkins, R., \& Singh, S. P. (2004). What are the benefits of evidence-based supported employment for patients with firstepisode psychosis?. Psychiatric Bulletin, 28(8), 281-284.

Robin, A. L., \& Weiss, J. G. (1980). Criterion-related validity of behavioral and selfreport measures of problem-solving communication skills in distressed and nondistressed parent-adolescent dyads. Behavioral Assessment, 2(4), 339-352. 
Robinson, D., Woerner, M. G., Alvir, J. M. J., Bilder, R., Goldman, R., Geisler, S., Koreen, A., Sheitman, B., Chakos, M., Mayerhoff, D., \& Lieberman, J. A. (1999). Predictors of relapse following response from a first episode of schizophrenia or schizoaffective disorder. Archives of General Psychiatry, 56(3), 241-247.

Robinson, D. G., Woerner, M. G., McMeniman, M., Mendelowitz, A., \& \& Bilder, R. M. (2004). Symptomatic and functional recovery from a first episode of schizophrenia or schizoaffective disorder. American Journal of Psychiatry, 161(3), 473-479.

Rodick, J. D., Henggeler, S. W., \& Hanson, C. L. (1986). An evaluation of the Family Adaptability and Cohesion Evaluation Scales and the Circumplex model. Journal of Abnormal Child Psychology, 14(1), 77-87.

Saunders, J. C. (2003). Families living with severe mental illness: A literature review. Issues in Mental Health Nursing, 24(2), 175-198.

Scazufca, M., \& Kuipers, E. (1996). Links between expressed emotion and burden of care in relatives of patients with schizophrenia. British Journal of Psychiatry, $168(5), 580-587$.

Shannon, C. E., \& Weaver, W. (1949). The mathematical theory of communication. Urbana: University of Illinois Press.

Shapiro, D. N., Chandler, J., \& Mueller, P. A. (2013). Using Mechanical Turk to study clinical populations. Clinical Psychological Science, 1(2), 213-220.

Sheehan, D. V. (2000). Sheehan Disability Scale. Handbook of Psychiatric Measures. Washington, DC: American Psychiatric Association, 113-115. 
Sheehan, D.V., Lecrubier, Y., Harnett-Sheehan, K., Janavs, J., Weiller, E., \& Bonora, L. I. (1997). Reliability and validity of the MINI International Neuropsychiatric Interview (M.I.N.I.): according to the SCID-P. European Psychiatry, 12, 232 241.

Sheehan, D.V., Lecrubier, Y., Harnett-Sheehan, K., et al. (1998). The Mini International Neuropsychiatric Interview (M.I.N.I.): the development and validation of a structured diagnostic psychiatric interview for DSM-IV and ICD10. Journal of Clinical Psychiatry, 59, (suppl 20), 22-33.

Smetanin, P., Stiff, D., Briante, C., Adair, C., Ahmad, S., \& Khan, M. (2011). The life and economic impact of major mental illnesses in Canada: 2011 to 2041. Retrieved from Mental Health Commission of Canada website: www.mentalhealthcommission.ca.

So, S. H., Freeman, D., Dunn, G., Kapur, S., Kuipers, E., Bebbington, P., ... \& Garety, P. A. (2012). Jumping to conclusions, a lack of belief flexibility and delusional conviction in psychosis: a longitudinal investigation of the structure, frequency, and relatedness of reasoning biases. Journal of Abnormal Psychology, 121(1), 129-139.

Stirling, J., Tantam, D., Thomas, P., Newby, D., Montague, L., Ring, N., \& Rowe, S. (1991). Expressed emotion and early onset schizophrenia: a 1 year follow-up. Psychological Medicine, 21(3), 675-685.

Stirling, J., Tantam, D., Thomas, P., Newby, D., Montague, L., Ring, N., \& Rowe, S. (1993). Expressed emotion and schizophrenia: the ontogeny of EE during an 18 month follow-up. Psychological Medicine, 23(3), 771-778. 
Strakowski, S. M., Keck, P. E., McElroy, S. L., Lonczak, H. S., \& West, S. A. (1995).

Chronology of comorbid and principal syndromes in first-episode psychosis. Comprehensive Psychiatry, 36(2), 106-112.

Szmukler, G. I., Burgess, P., Herrman, H., Benson, A., Colusa, S., \& Bloch, S. (1996). Caring for relatives with serious mental illness: the development of the Experience of Caregiving Inventory. Society of Psychiatry and Psychiatric Epidemiology, 31, $137-148$.

Tennakoon, L., Fannon, D., Doku, V., O’Ceallaigh, S., Soni, W., Santamaria, M., Kuipers, E., \& Sharma, T. (2000). Experience of caregiving: relatives of people experience a first episode of psychosis. British Journal of Psychiatry, 177, 529533.

Tien, A.Y. (1991). Distributions of hallucinations in the population. Social Psychiatry and Psychiatric Epidemiology, 26(6), 287-292.

Treasure, J., Murphy, T., Szmukler, G., Gavan, T. K., \& Joyce, J. (2001). The experience of caregiving for severe mental illness: a comparison between anorexia nervosa and psychosis. Society of Psychiatry and Psychiatric Epidemiology, 36, 343-347.

Tucker, C., Barker, A., \& Gregoire, A. (1998). Living with schizophrenia: caring for a person with a severe mental illness. Society of Psychiatry and Psychiatric Epidemiology, 33, 305-309.

Van der Giessen, D., Branje, S., Keijsers, L., Van Lier, P. A., Koot, H. M., \& Meeus, W. (2014). Emotional variability during mother-adolescent conflict interactions: Longitudinal links to adolescent disclosure and maternal control. Journal of Adolescence, 37(1), 23-31. 
Van der Giessen, D., Hollenstein, T., Hale III, W. W., Koot, H. M., Meeus, W., \& Branje, S. (2015). Emotional variability in mother-adolescent conflict interactions and internalizing problems of mothers and adolescents: dyadic and individual processes. Journal of Abnormal Child Psychology, 43(2), 339-353.

van Os, J., Hanssen, M., Bijl, R. V., \& Ravelli, A. (2000). Strauss (1969) revisited: a psychosis continuum in the general population? Schizophrenia Research, 45(1-2), $11-20$.

van Os J, Hanssen M, Bijl RV, Vollebergh W. (2001). Prevalence of psychotic disorder and community level of psychotic symptoms: an urban-rural comparison. Archives of General Psychiatry, 58(7), 663-668.

Vaughn, C.E. (1989). Expressed emotion in family relationships. Journal of Child Psychology and Psychiatry, 30(1), 13-22.

Widiger, T. A., \& Samuel, D. B. (2005). Diagnostic categories or dimensions? A question for the Diagnostic and statistical manual of mental disorders. Journal of Abnormal Psychology, 114(4), 494-504.

Wykes, T., Newton, E., Landau, S., Rice, C., Thompson, N., \& Frangou, S. (2007). Cognitive remediation therapy (CRT) for young early onset patients with schizophrenia: an exploratory randomized controlled trial. Schizophrenia Research, 94(1), 221-230.

Yung, A. R., McGorry, P. D., McFarlane, C. A., Jackson, H. J., Patton, G. C., \& Rakkar, A. (1996). Monitoring and care of young people at incipient risk of psychosis. Schizophrenia Bulletin, 22, 283-303. 


\section{Appendix A}

The following table presents data from an independent $t$-test analysis between survey respondents that were included or excluded from the caregiver survey study presented in Chapter 2. Exclusion criteria included incomplete survey data, inconsistent responding, or completing the survey in less than 11 minutes.

\section{Appendix A Table}

Differences between survey respondents who were included or excluded from the caregiver survey study

\begin{tabular}{|c|c|c|c|c|c|}
\hline & \multicolumn{2}{|c|}{ Included } & \multicolumn{2}{|c|}{ Excluded } & \multirow[b]{2}{*}{$p$} \\
\hline & $n$ & $M(S D)$ & $n$ & $M(S D)$ & \\
\hline \multicolumn{6}{|l|}{ Caregiver } \\
\hline Age (years) & 106 & $46.42(11.86)$ & 66 & $33.83(10.45)$ & $<.001$ \\
\hline Number of children & 107 & $2.07(1.34)$ & 66 & $1.55(0.19)$ & .001 \\
\hline \multicolumn{6}{|l|}{ Family member with psychosis } \\
\hline Age (years) & 107 & $23.43(6.48)$ & 40 & $23.43(9.58)$ & .501 \\
\hline Age of illness onset (years) & 106 & $17.84(6.37)$ & 41 & $34.39(21.13)$ & $<.001$ \\
\hline Duration psychosis was untreated (months) $\phi$ & 101 & $17.45(28.22)$ & 40 & $13.80(16.14)$ & .444 \\
\hline Duration in treatment (months) & 105 & $33.20(23.83)$ & 40 & $20.93(20.12)$ & .004 \\
\hline Age at first psychiatric hospitalization & 76 & $19.71(5.65)$ & 17 & $28.94(14.80)$ & $<.001$ \\
\hline Total number of psychiatric hospitalizations & 75 & $4.08(5.03)$ & 17 & $4.47(4.33)$ & .768 \\
\hline Time since last hospitalization (months) & 75 & $23.89(22.47)$ & 17 & $18.71(17.89)$ & .376 \\
\hline \multicolumn{6}{|l|}{ Caregiver survey measures } \\
\hline Family Functioning DFC & 101 & $10.40(7.15)$ & 31 & $8.81(1.58)$ & .129 \\
\hline $\begin{array}{l}\text { ECI Positive } \\
\text { Possible range }=0-4 \\
\text { Higher scores indicate more positive appraisals }\end{array}$ & 106 & $2.19(0.55)$ & 30 & $2.12(0.86)$ & .616 \\
\hline $\begin{array}{l}\text { ECI Negative } \\
\text { Possible range }=0-4 \\
\text { Higher scores indicate more negative appraisals }\end{array}$ & 106 & $1.90(0.62)$ & 31 & $1.78(0.64)$ & .379 \\
\hline
\end{tabular}




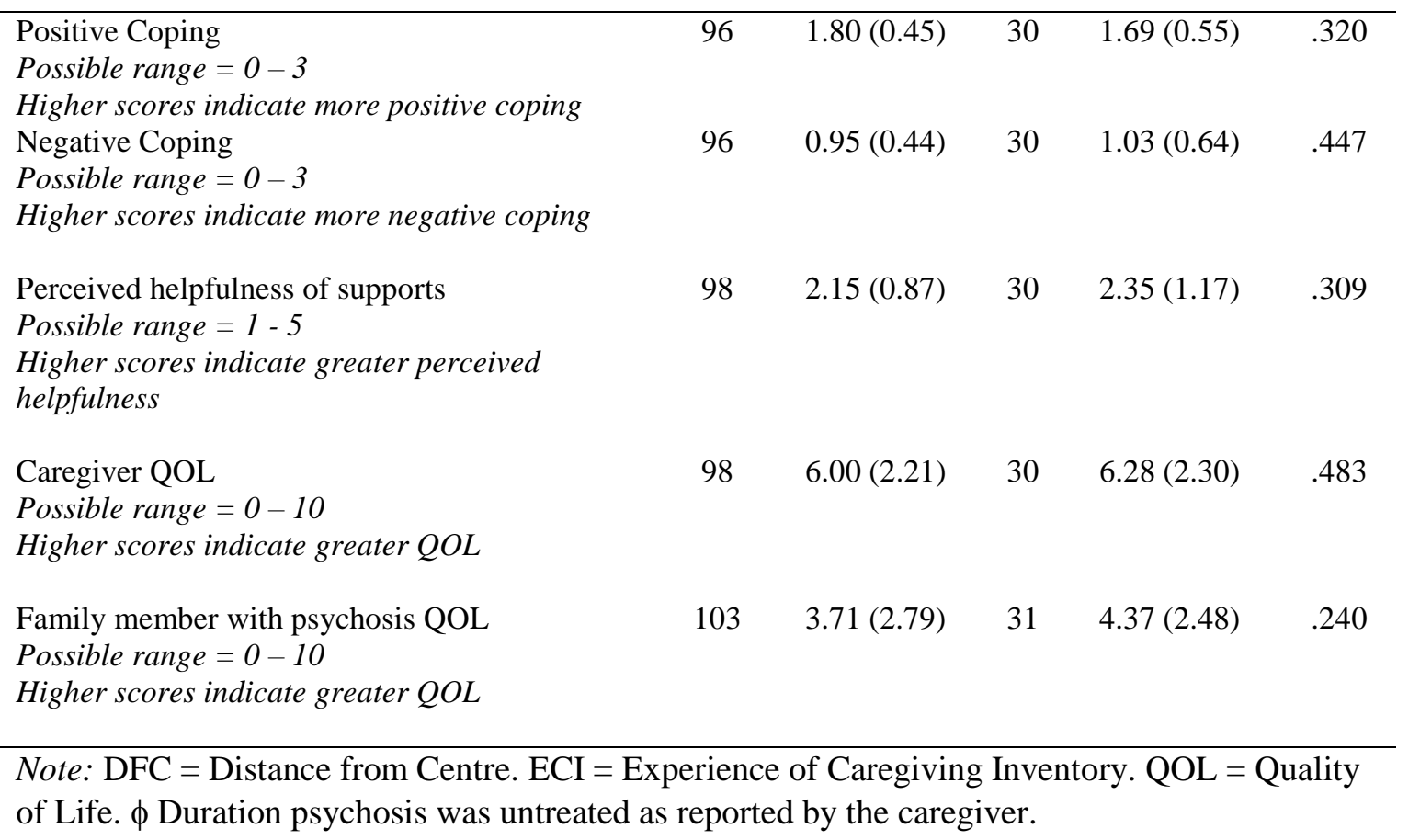




\section{Appendix B}

Healthy Control Family 1 (HC1)

HC1, First Positive Discussion

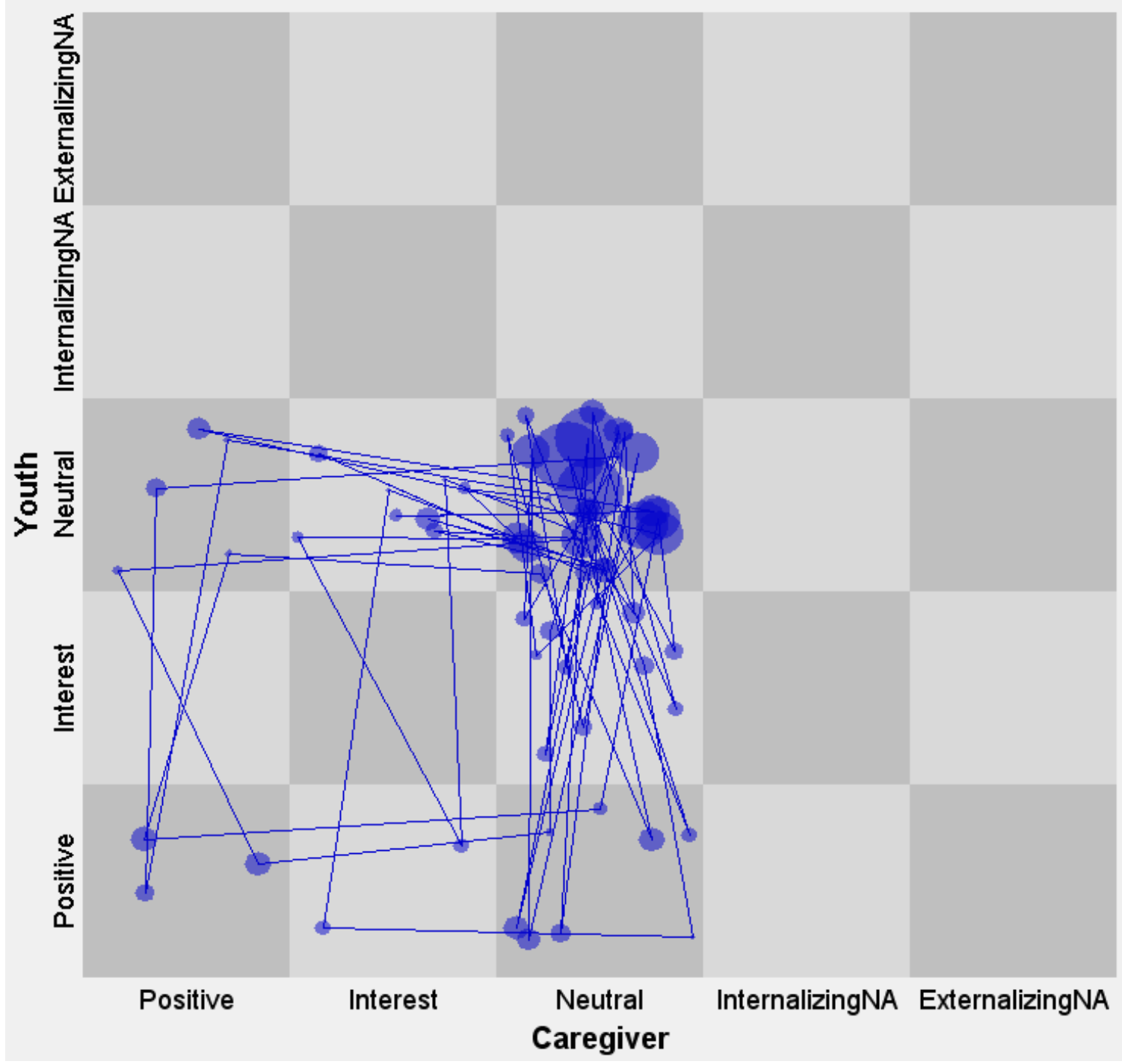


HC1, Normative Conflict Discussion

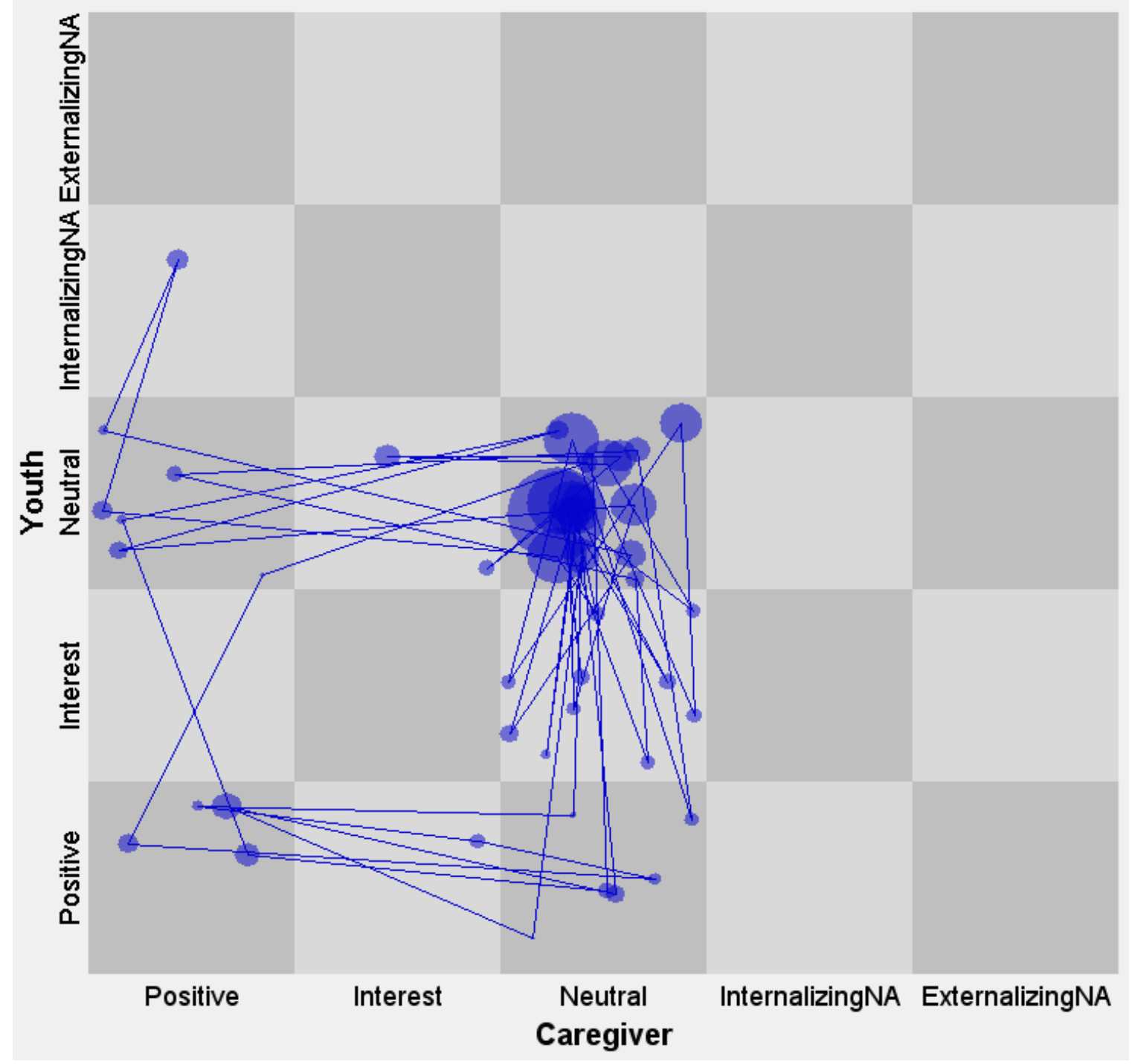


HC 1, Second Positive Discussion

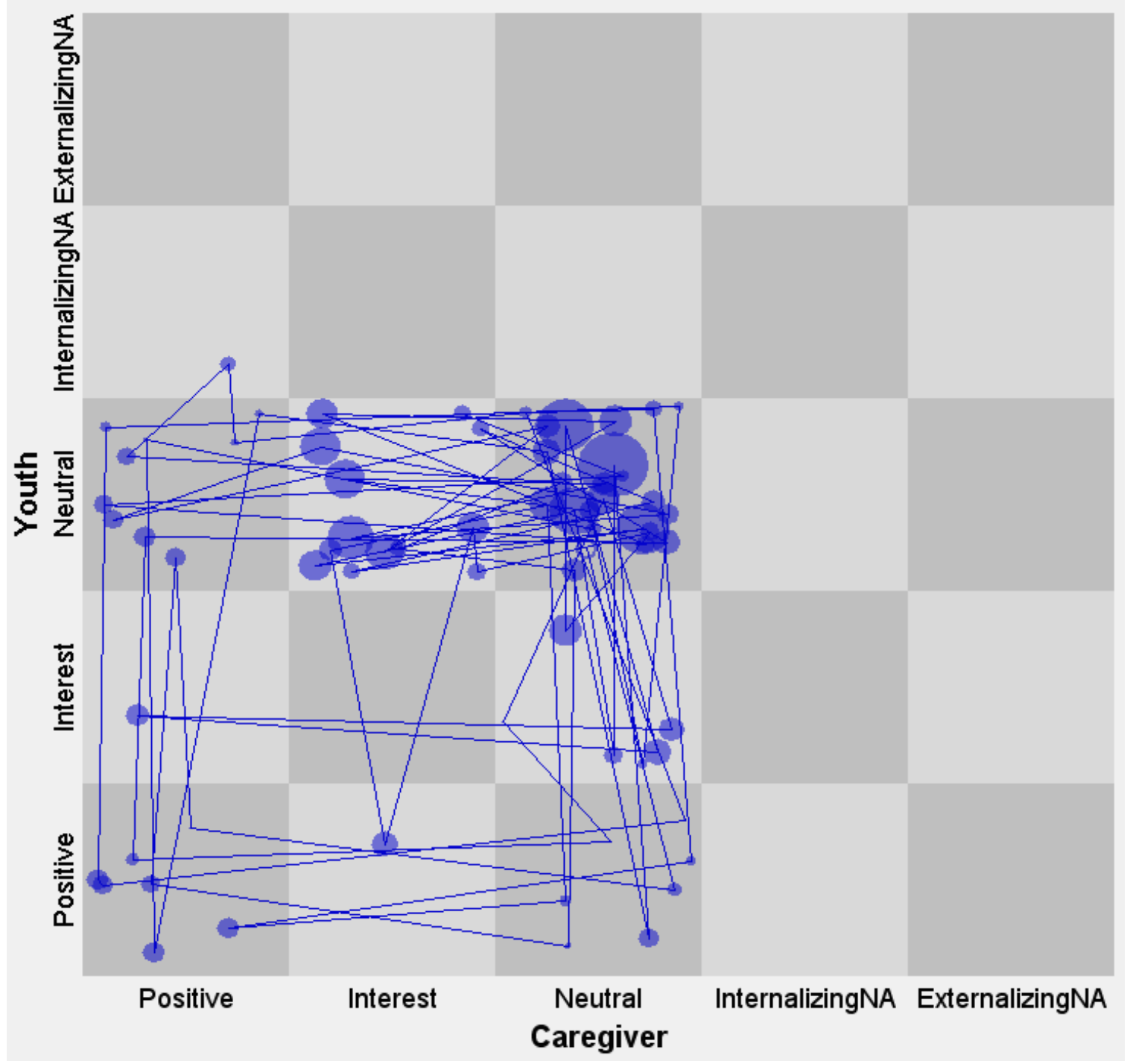


Healthy Control Family 2 (HC2)

HC2, First Positive Discussion

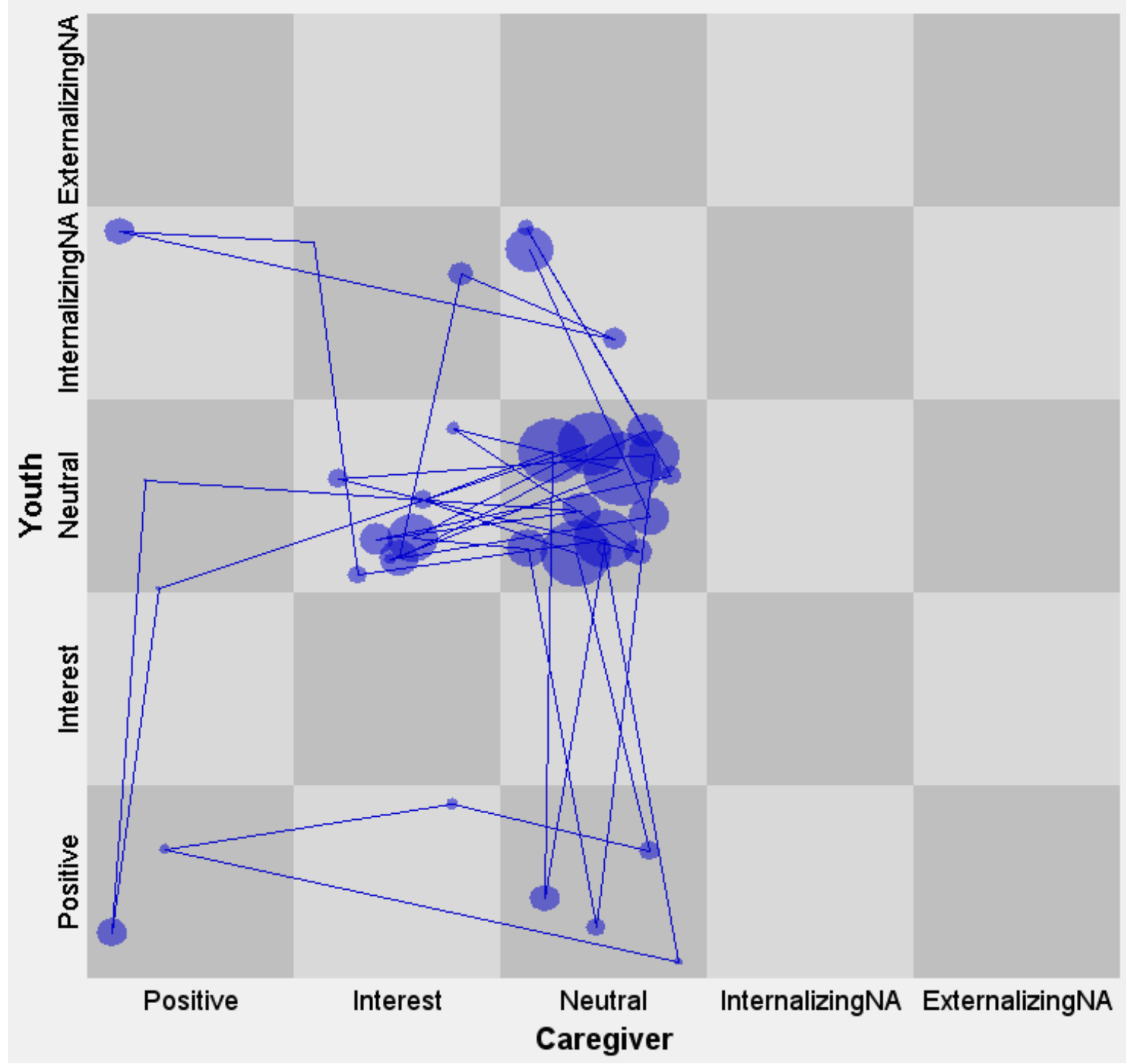


HC2, Normative Conflict Discussion

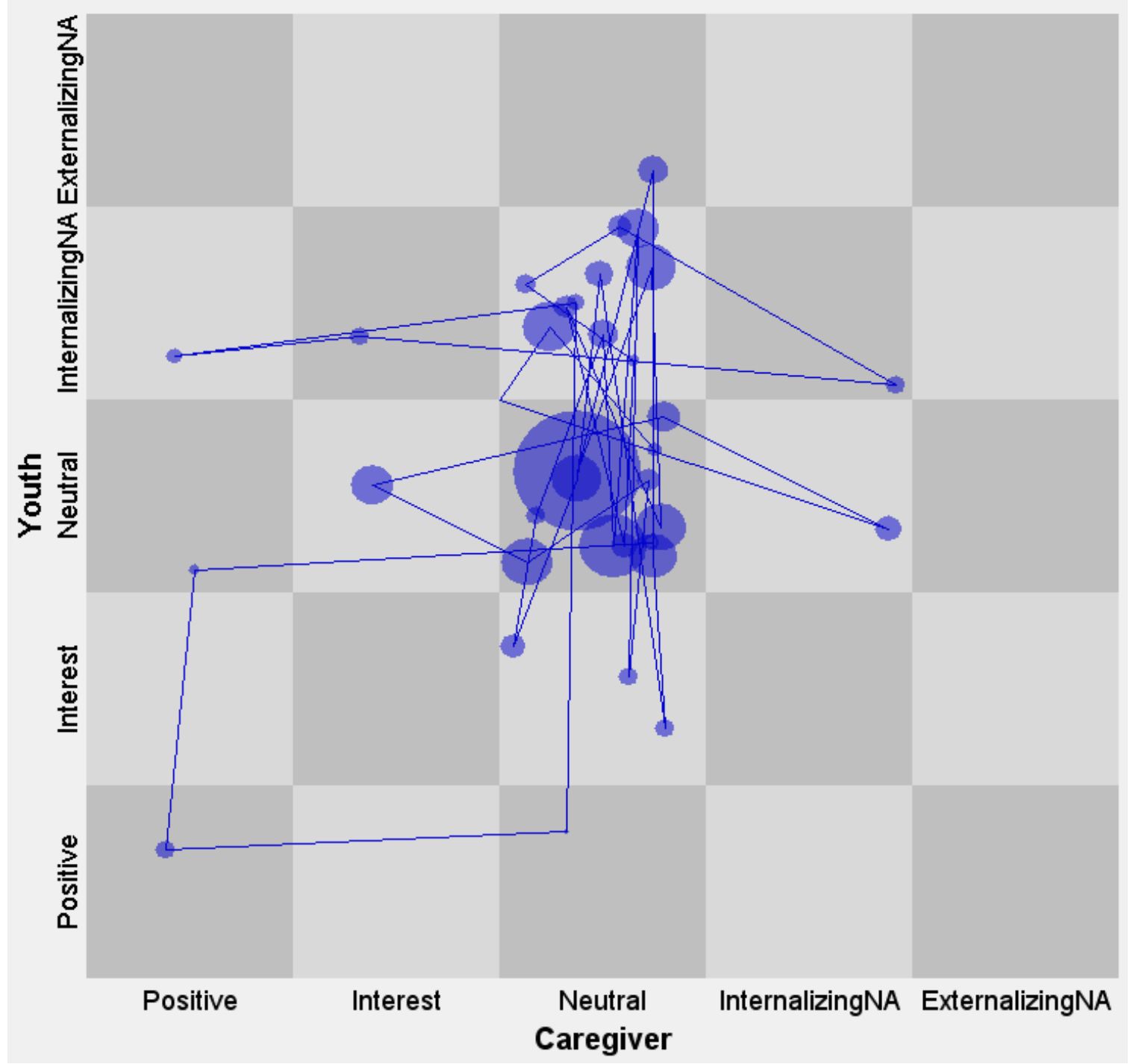


HC2, Second Positive Discussion

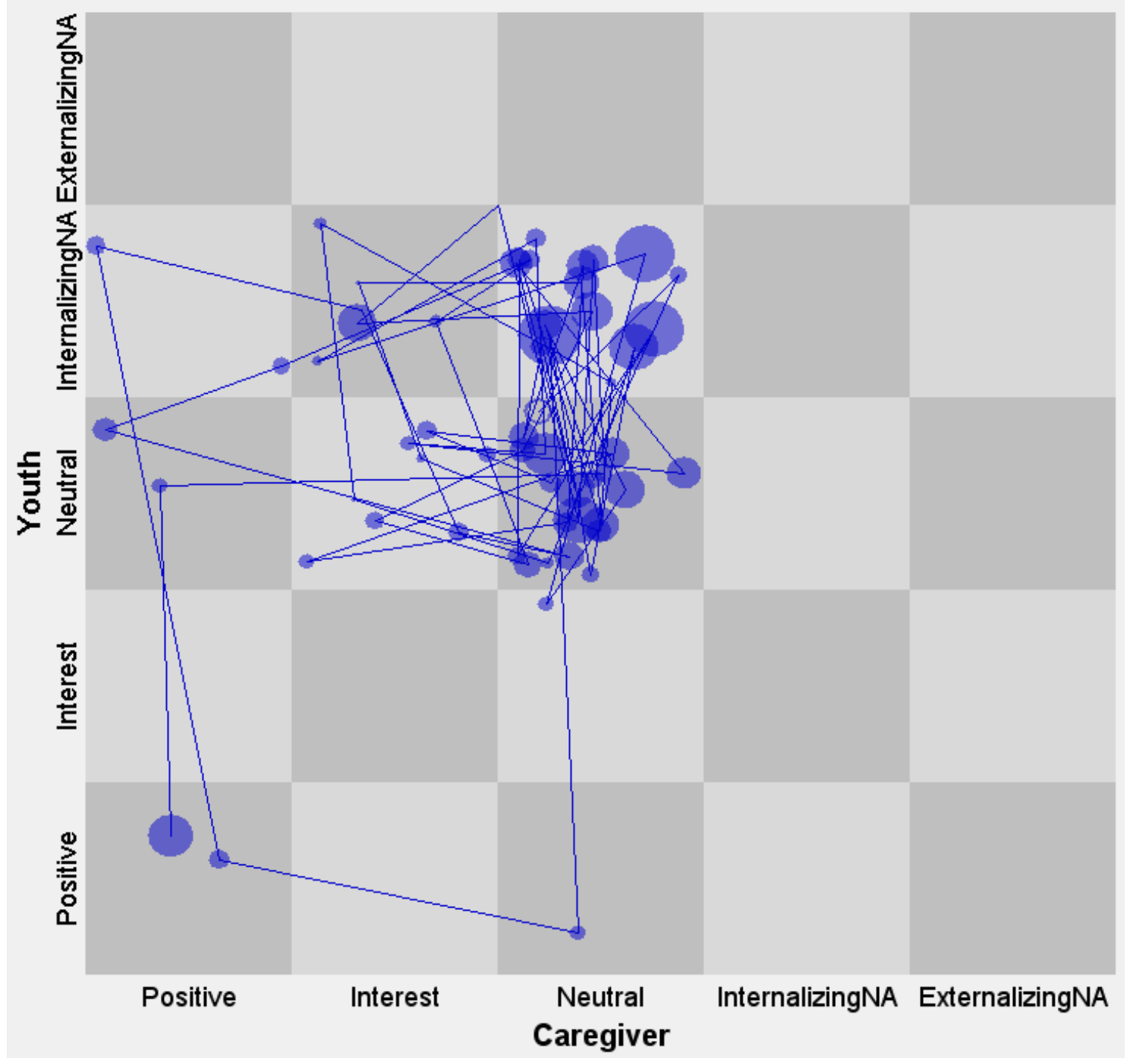


Healthy Control Family 3 (HC3)

HC3, First Positive Discussion

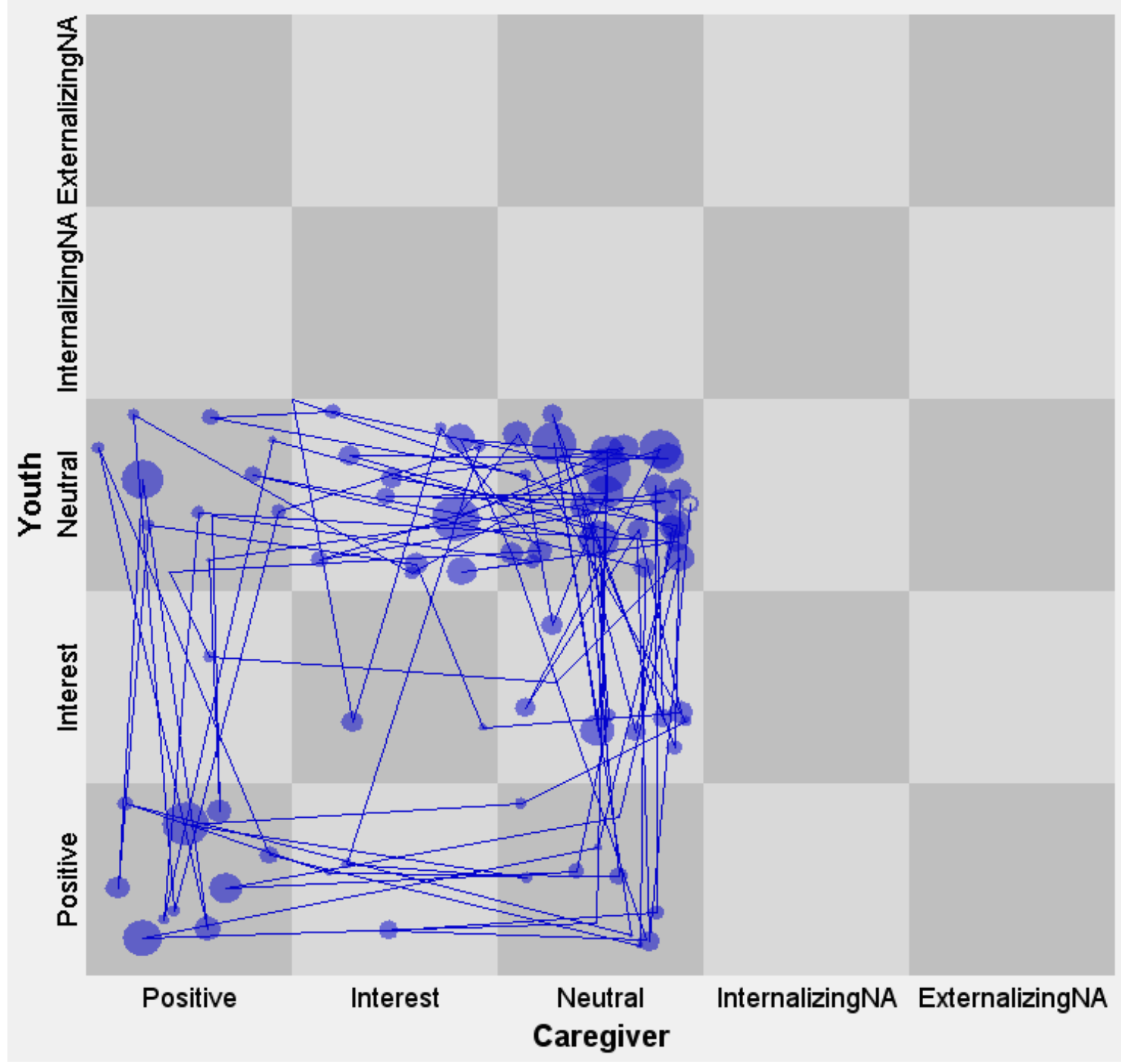


HC3, Normative Conflict Discussion

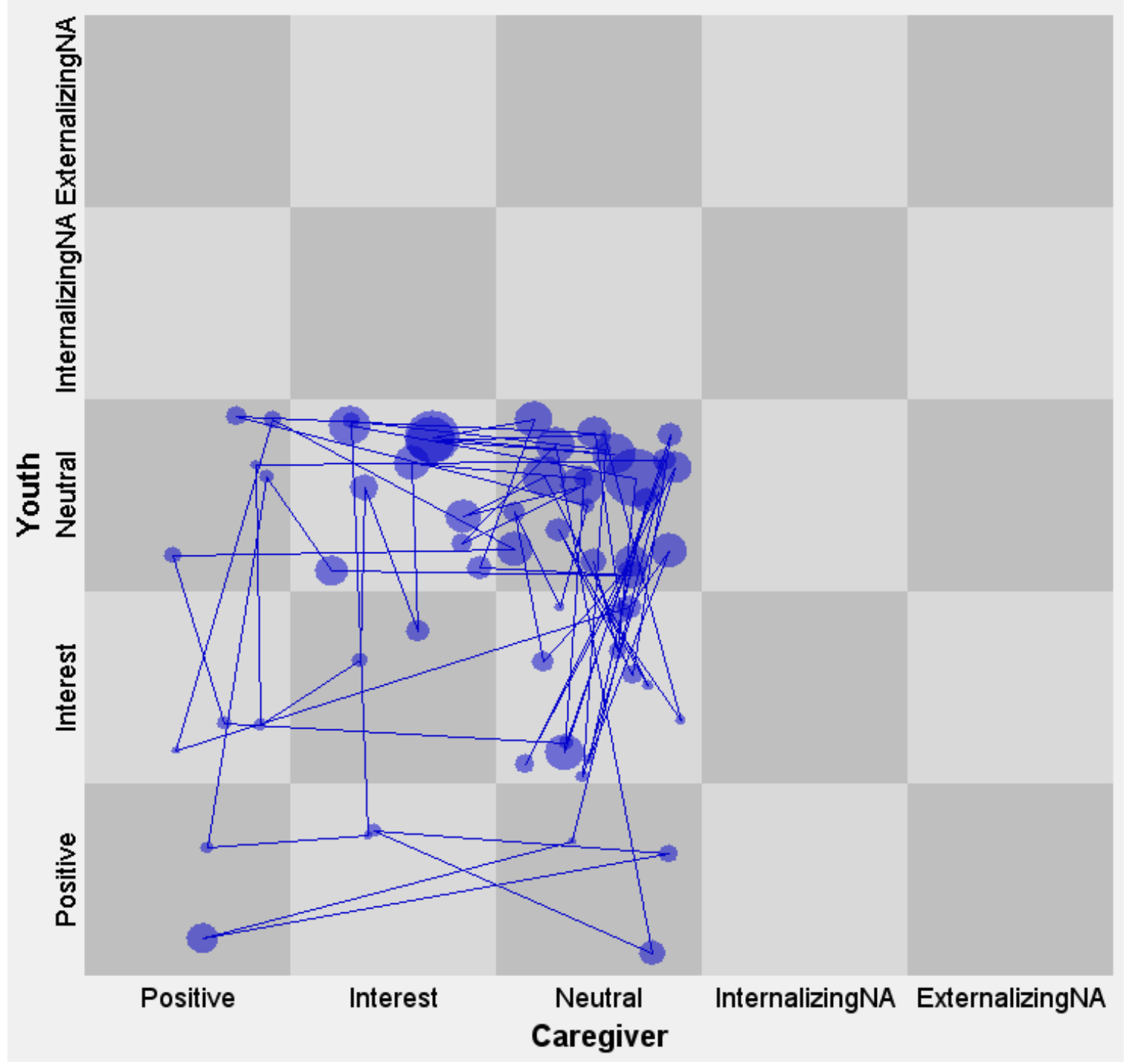


HC3, Second Positive Discussion

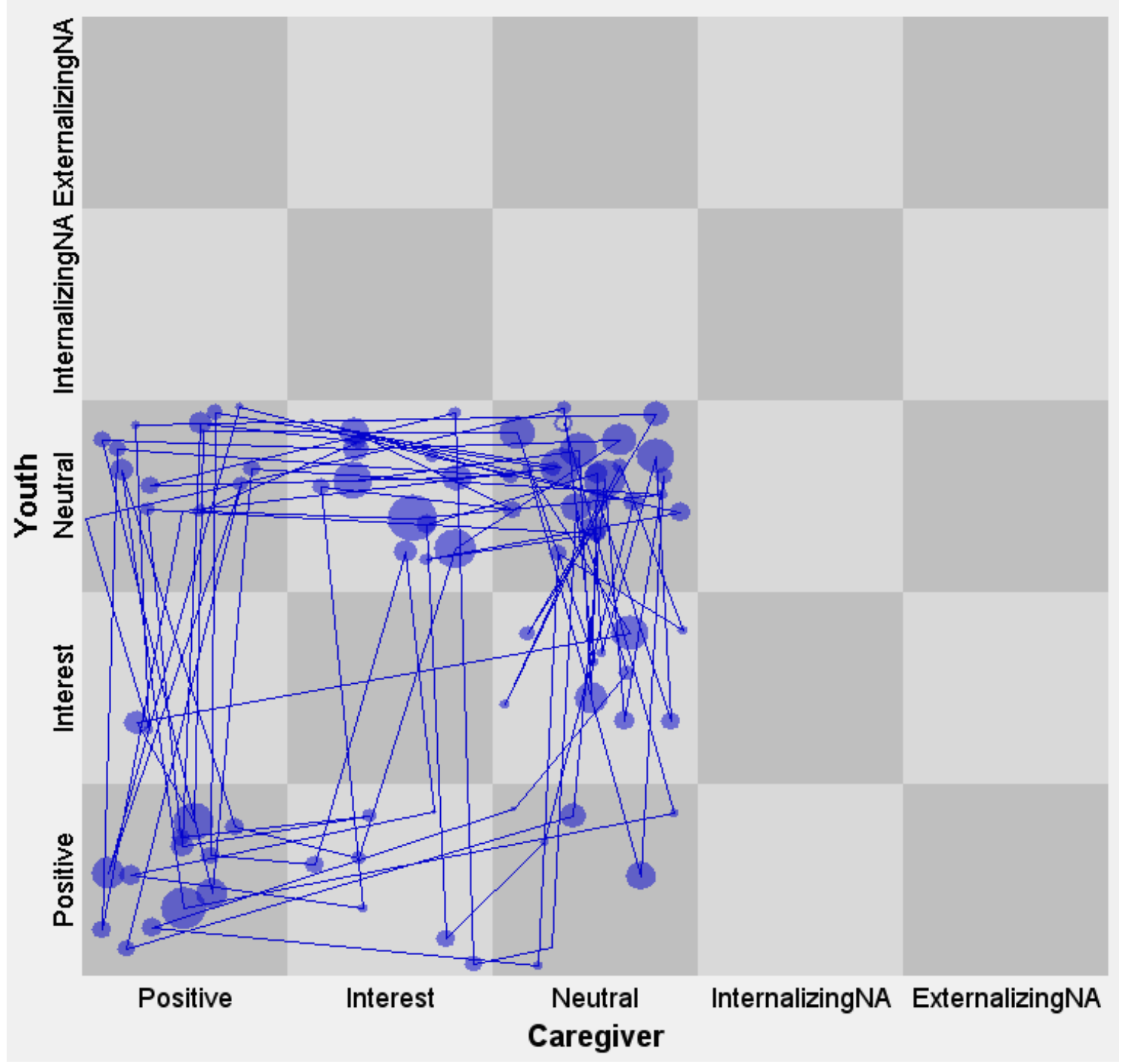


Healthy Control Family 4 (HC4)

HC4, First Positive Discussion

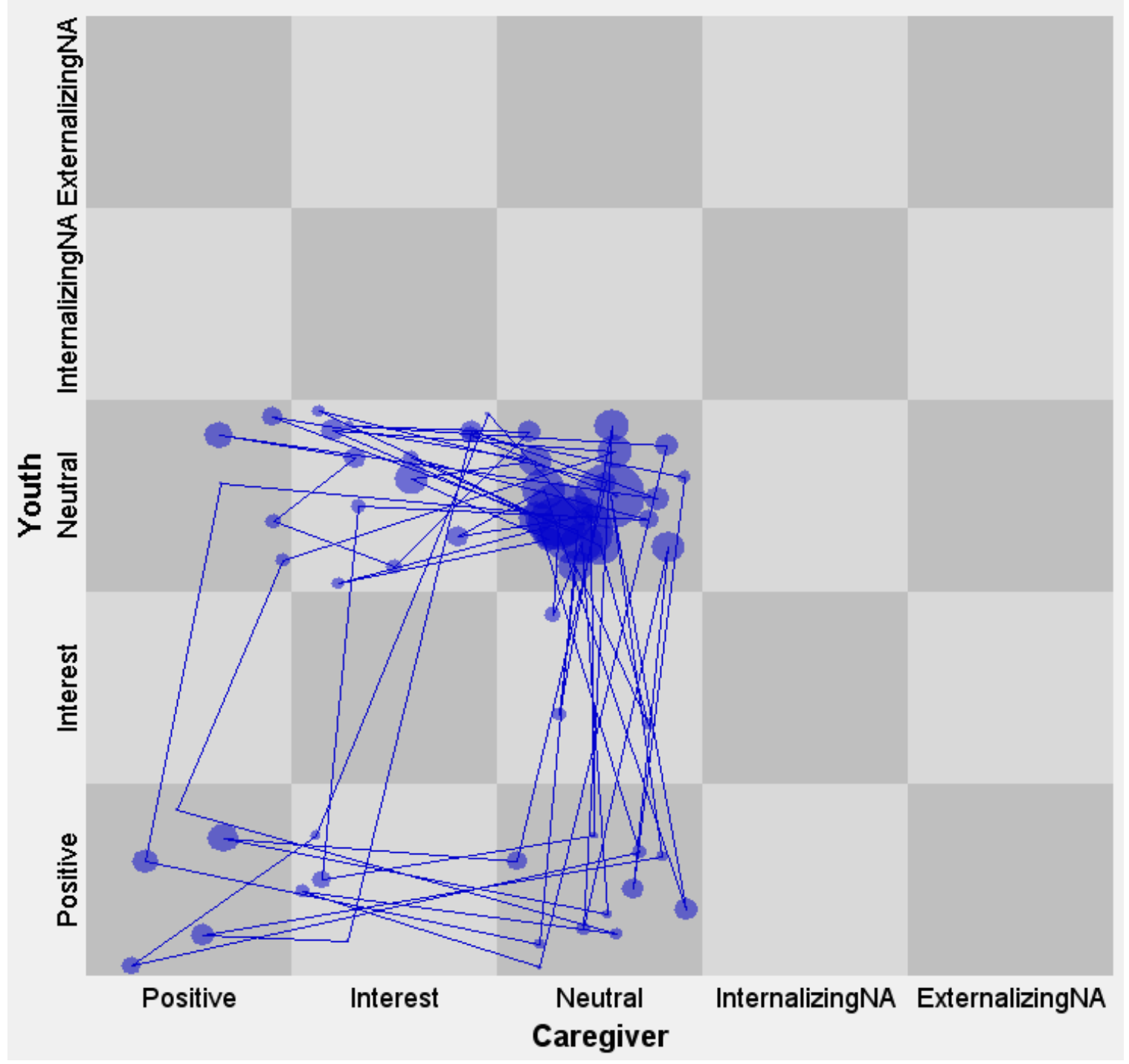


HC4, Normative Conflict Discussion

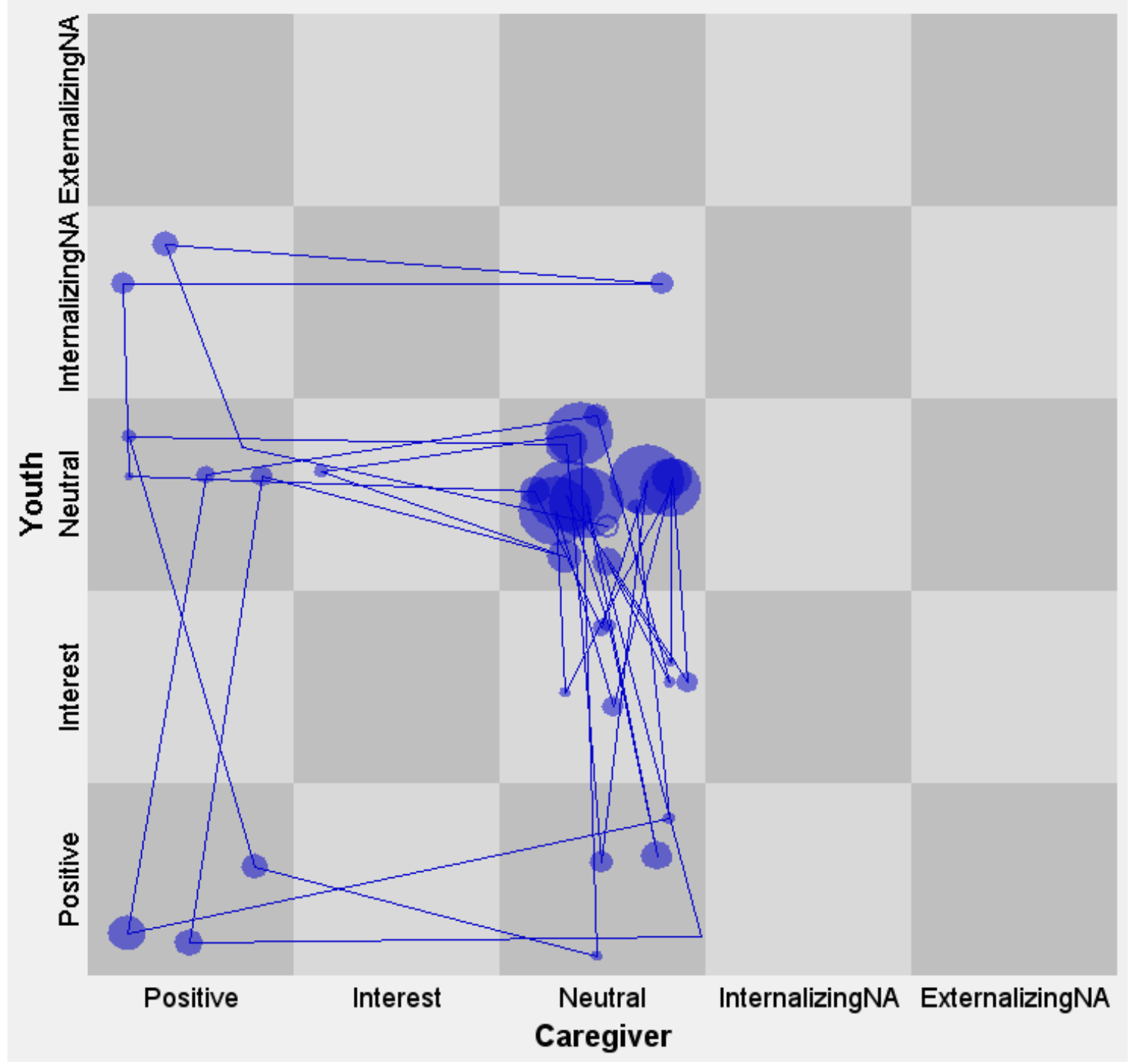


HC4, Second Positive Discussion

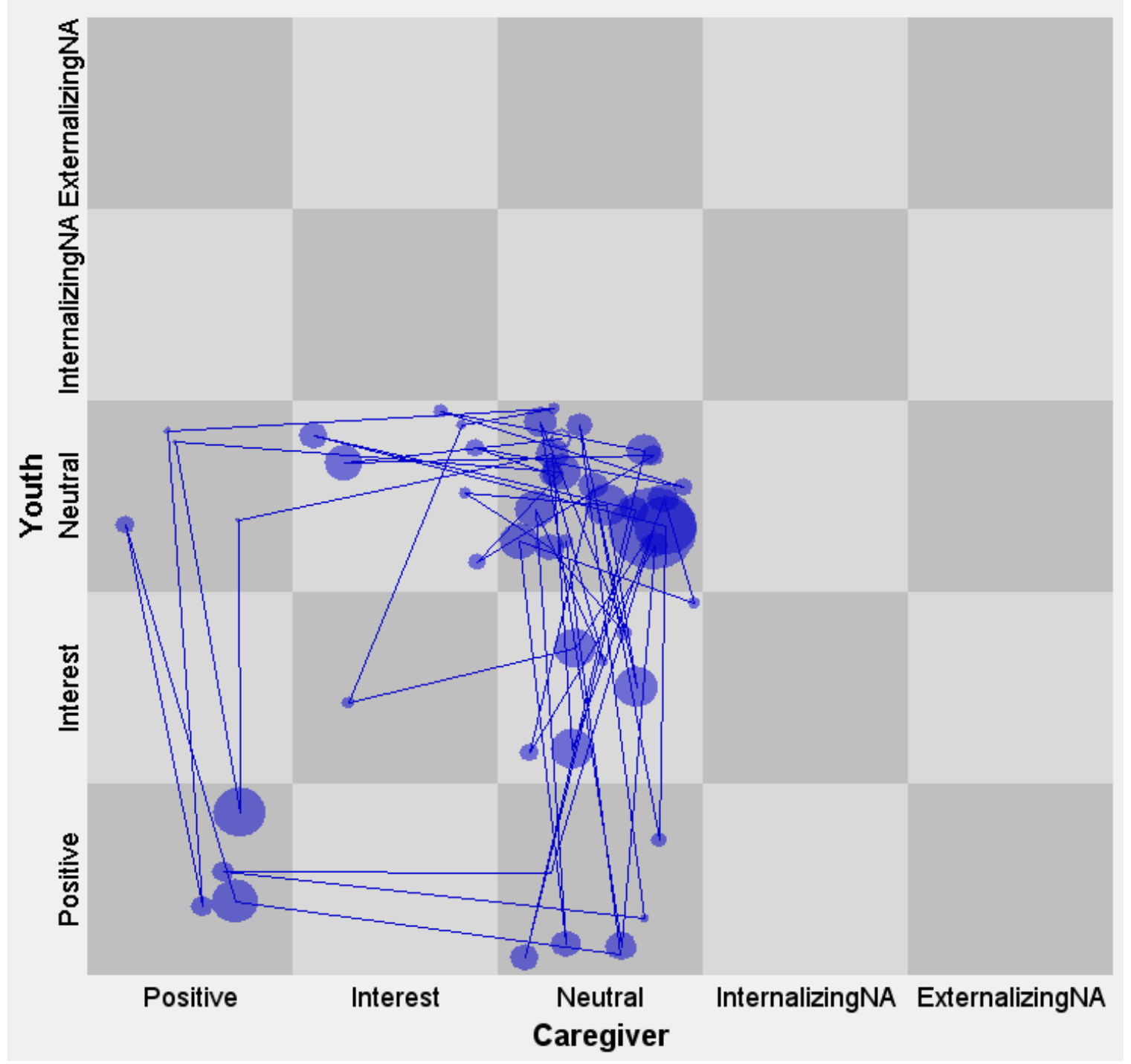


Healthy Control Family 5 (HC5)

HC5, First Positive Discussion

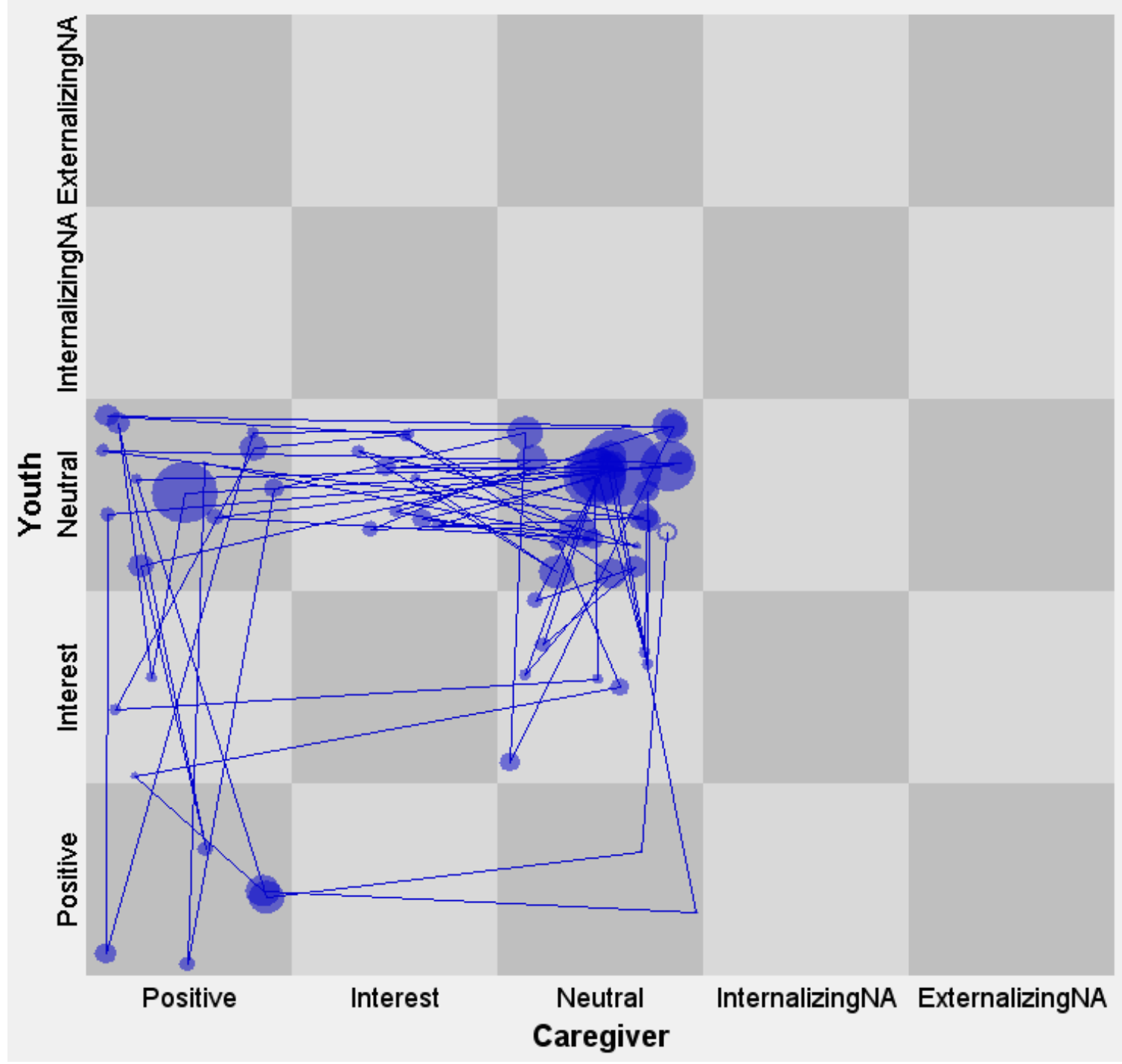


HC5, Normative Conflict Discussion

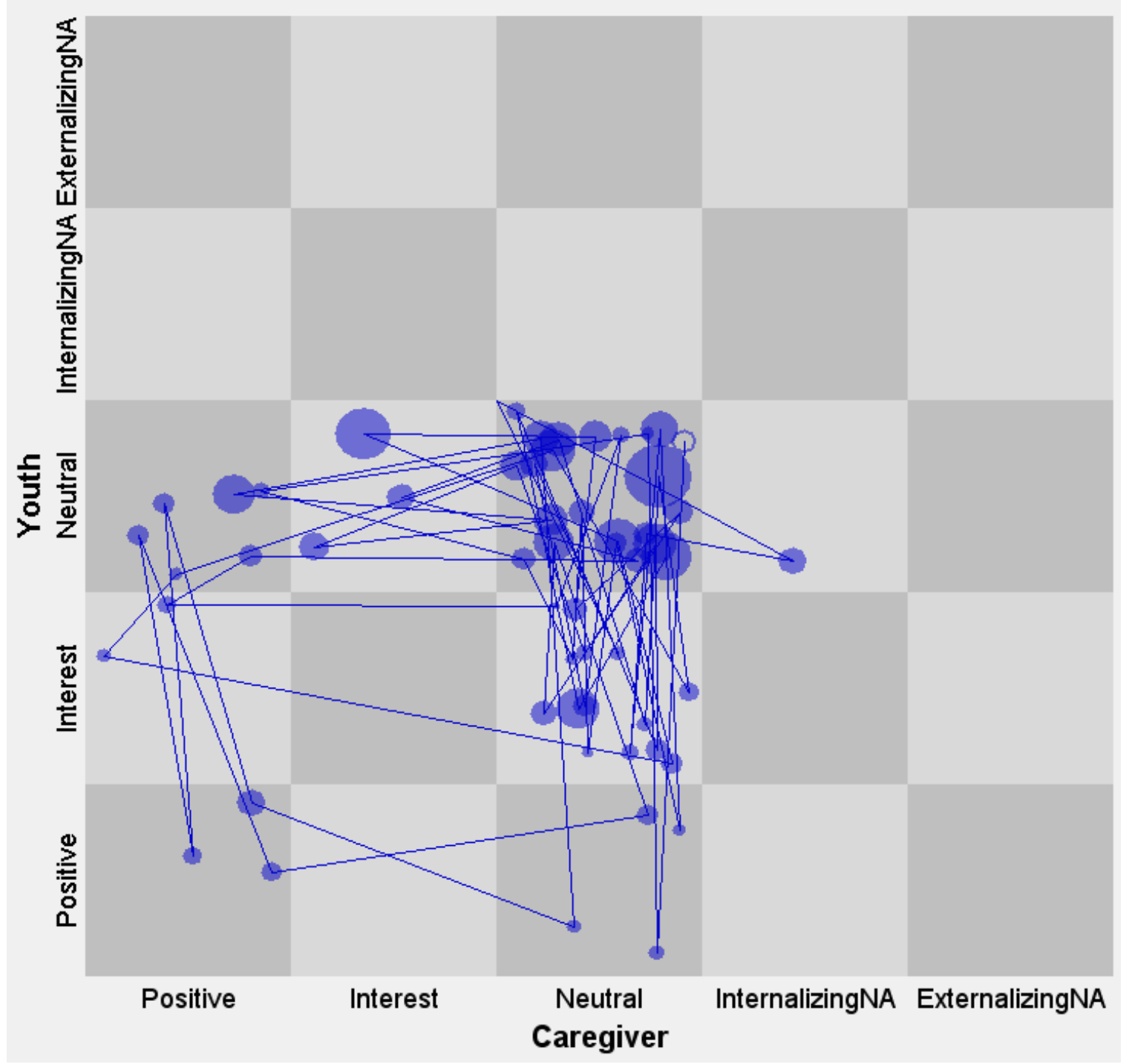


HC5, Second Positive Discussion

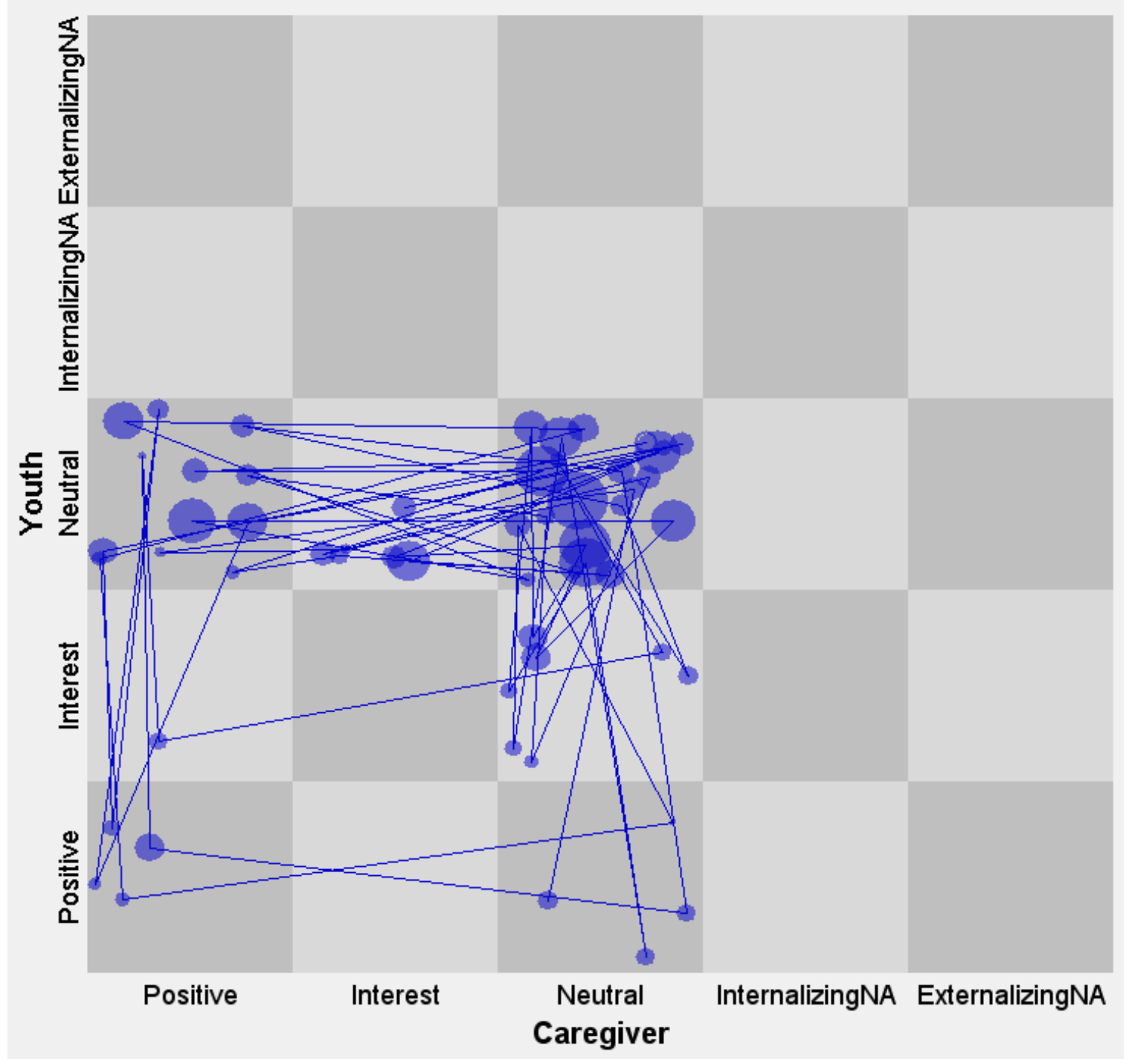


Family with an Individual with Psychosis 1 (PSY1)

PSY1, First Positive Discussion

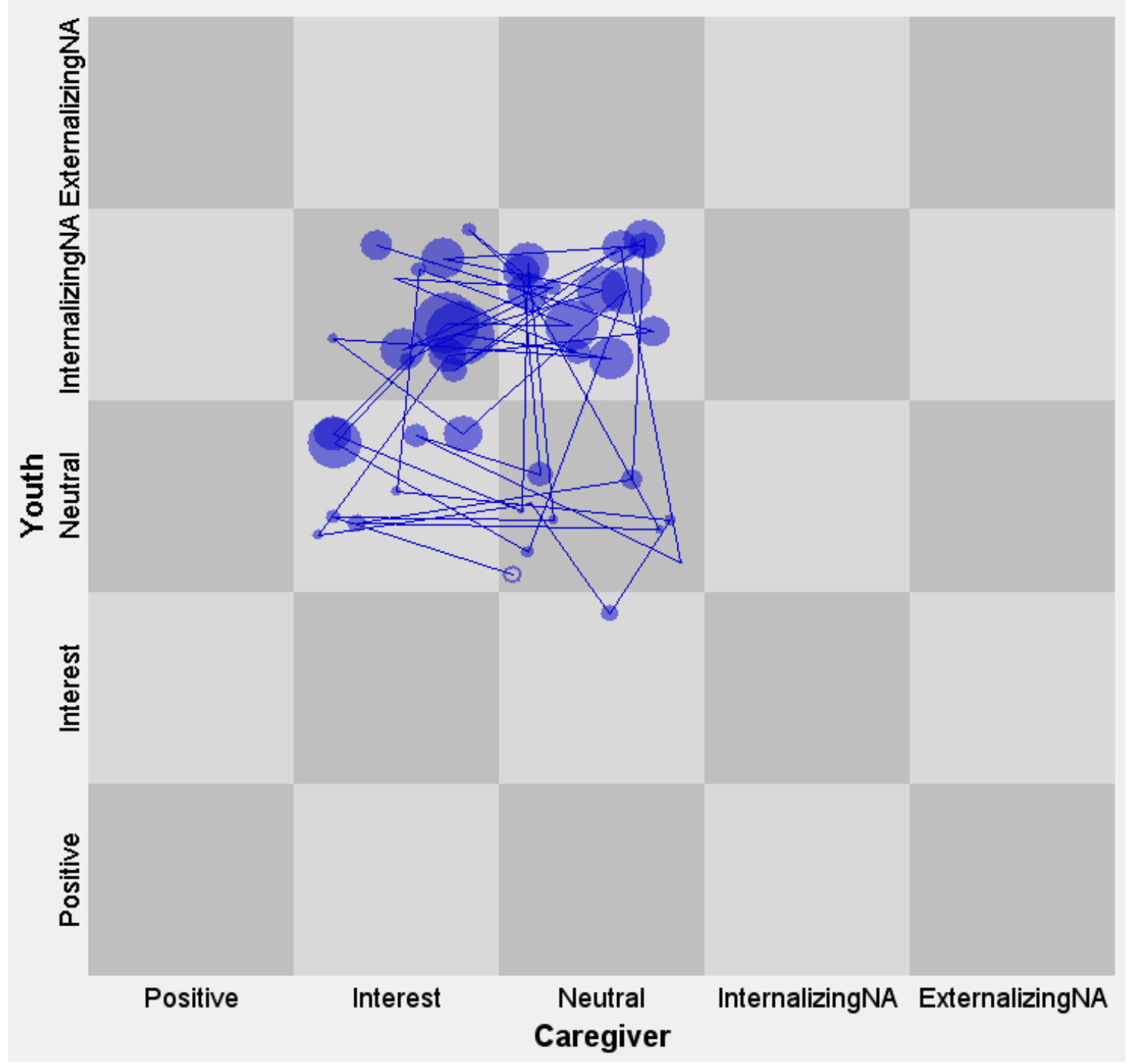


PSY1, Normative Conflict Discussion

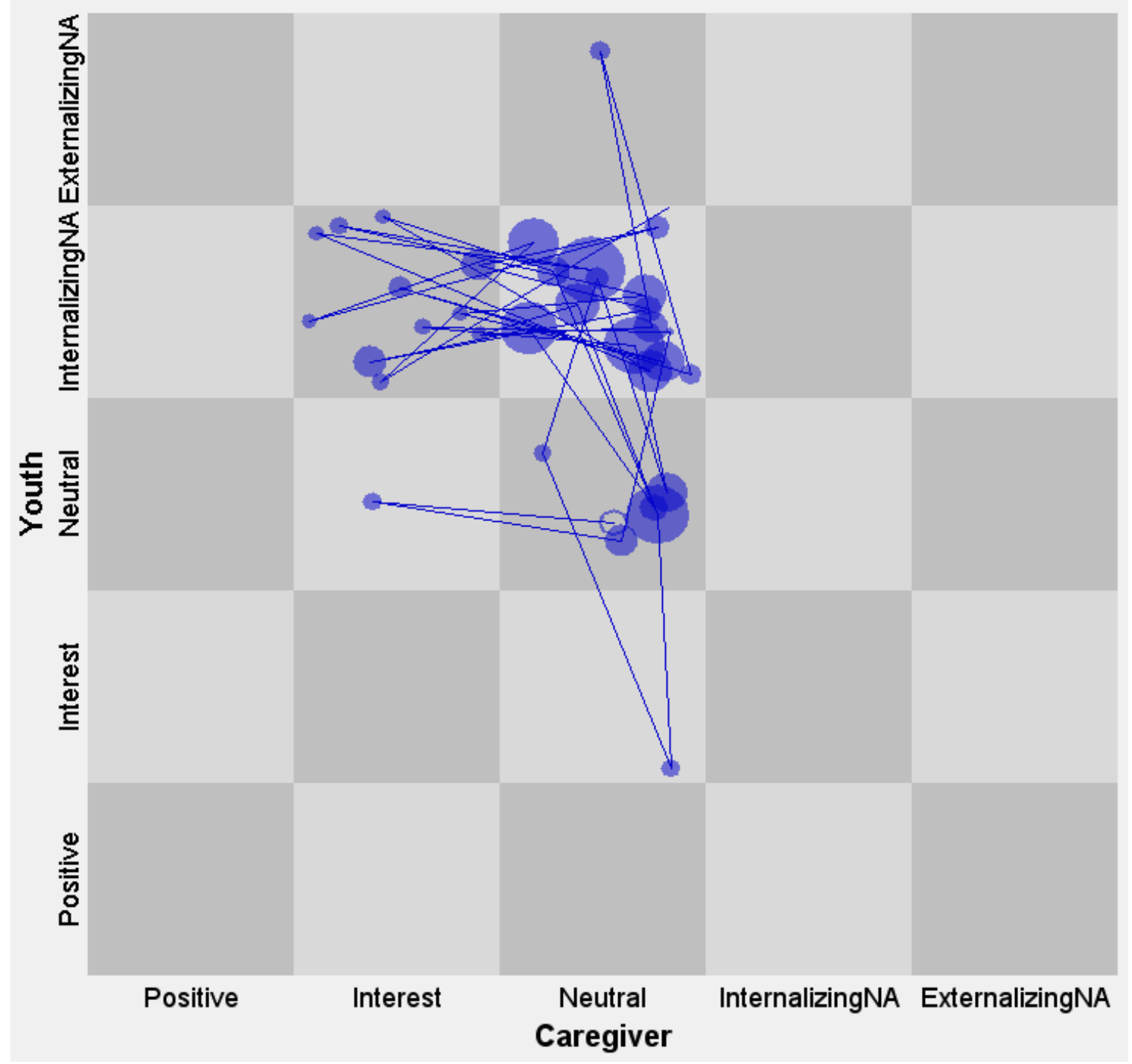


PSY1, Second Positive Discussion

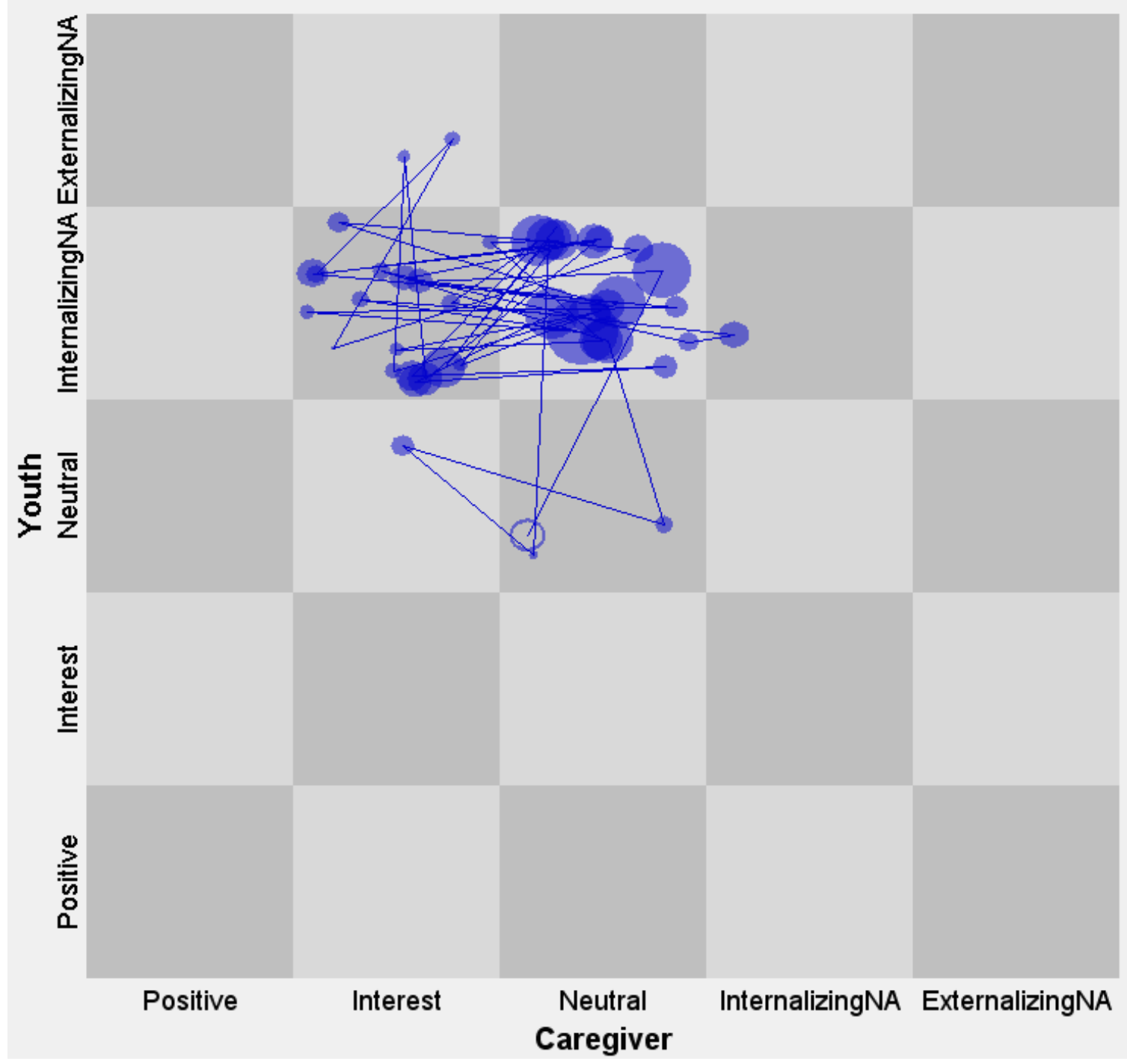


PSY1, Psychosis-Specific Conflict Discussion

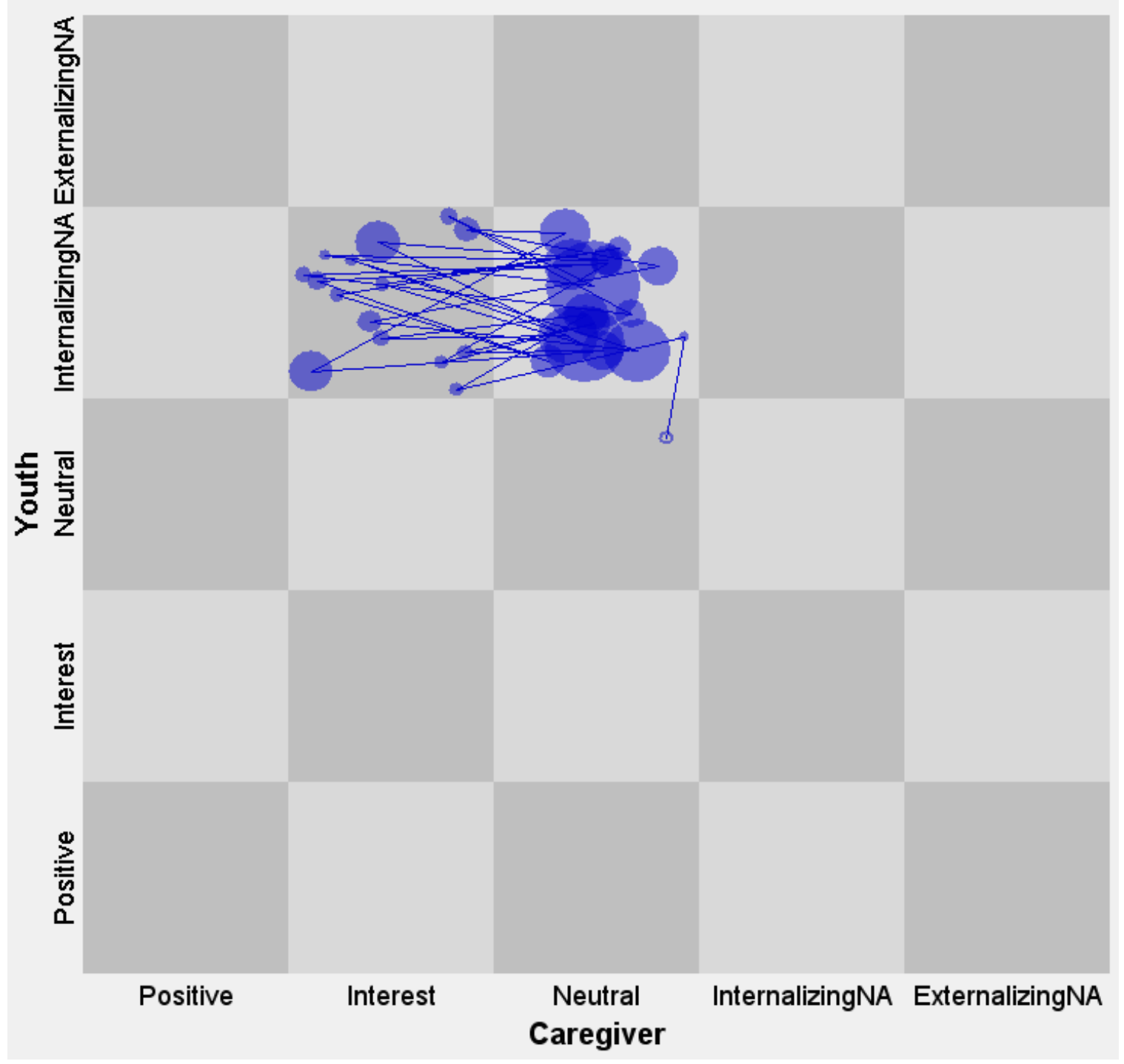


PSY1, Third Positive Discussion

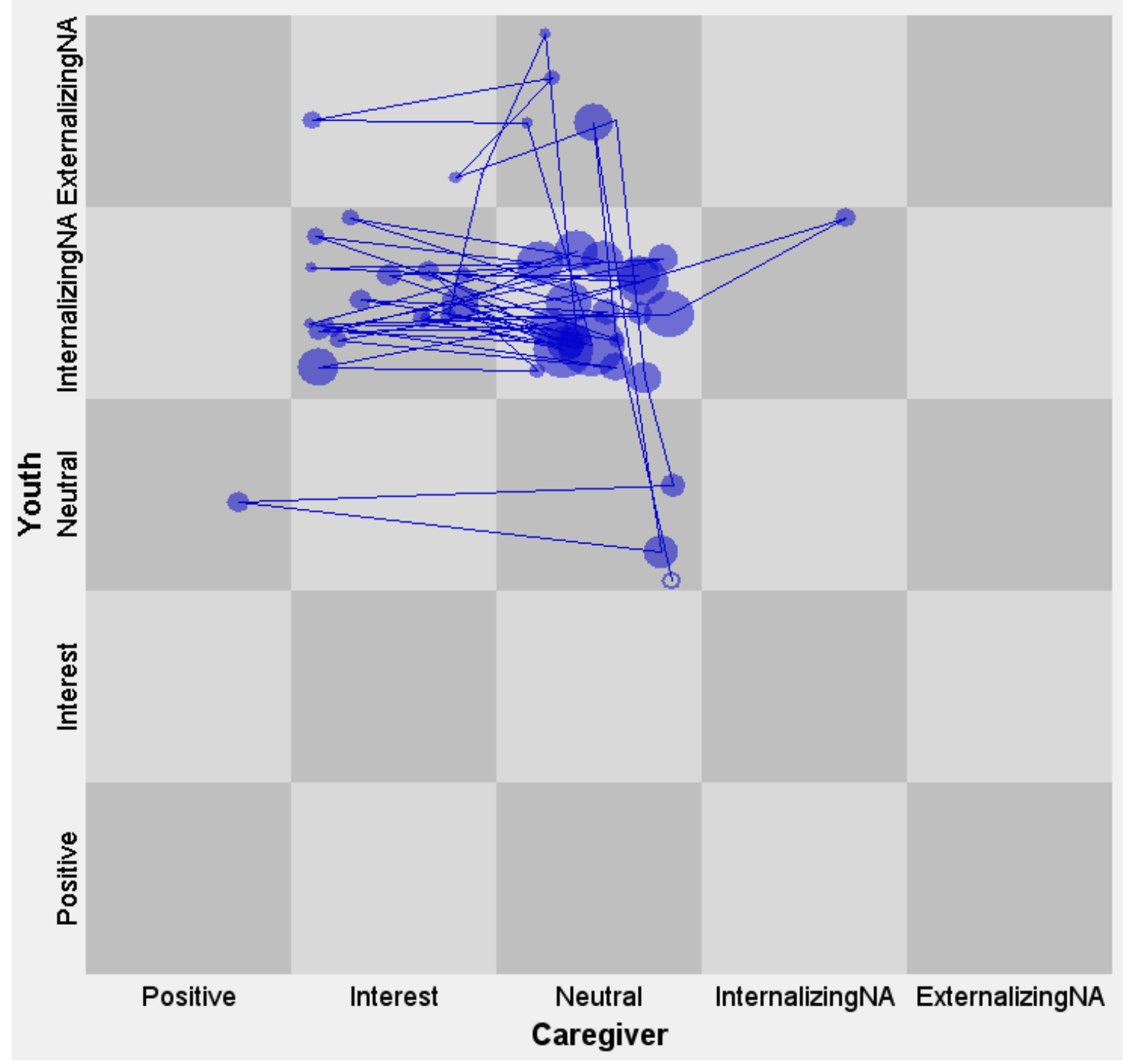


Family with an Individual with Psychosis 2 (PSY2)

PSY2, First Positive Discussion

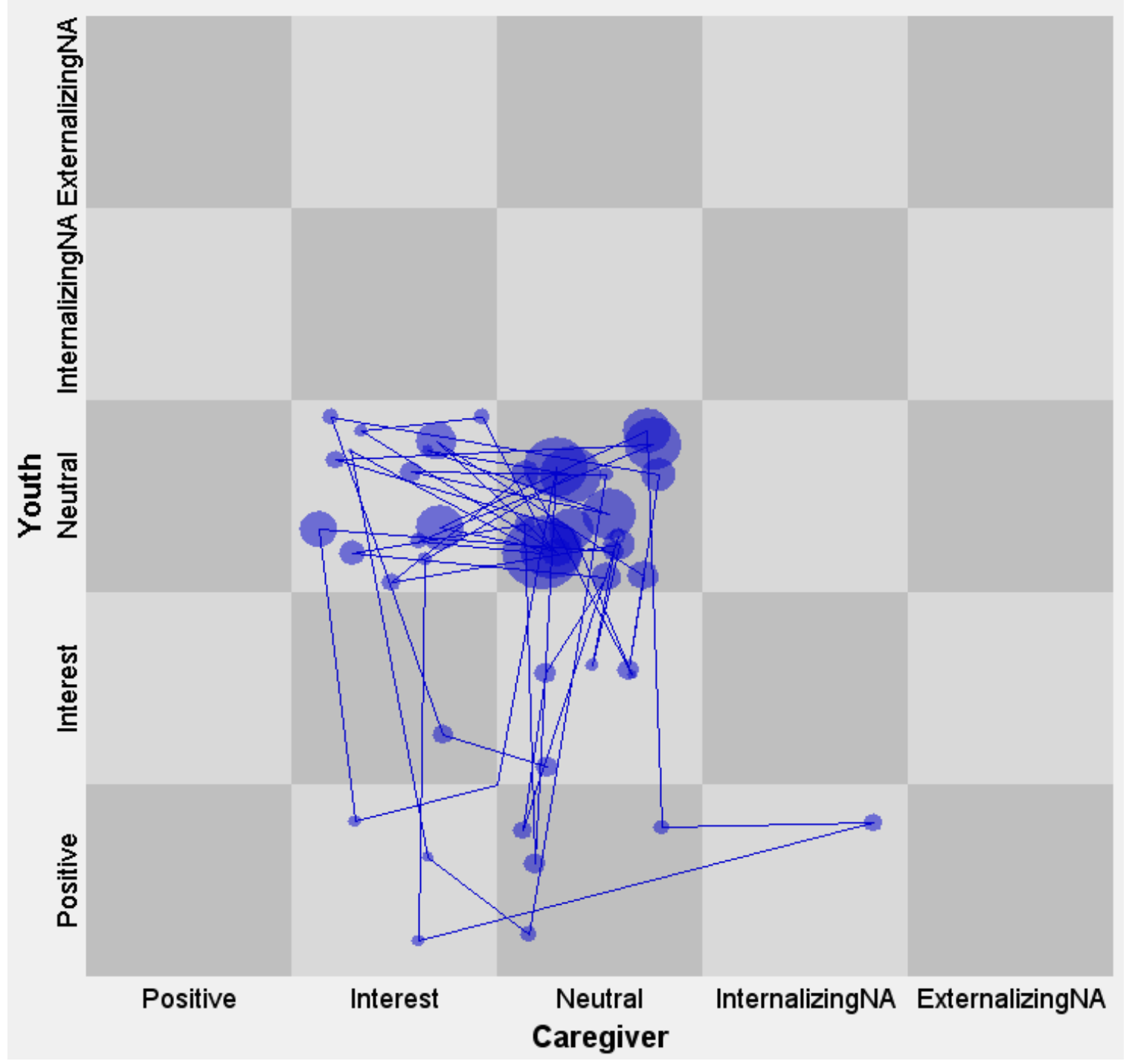


PSY2, Normative Conflict Discussion

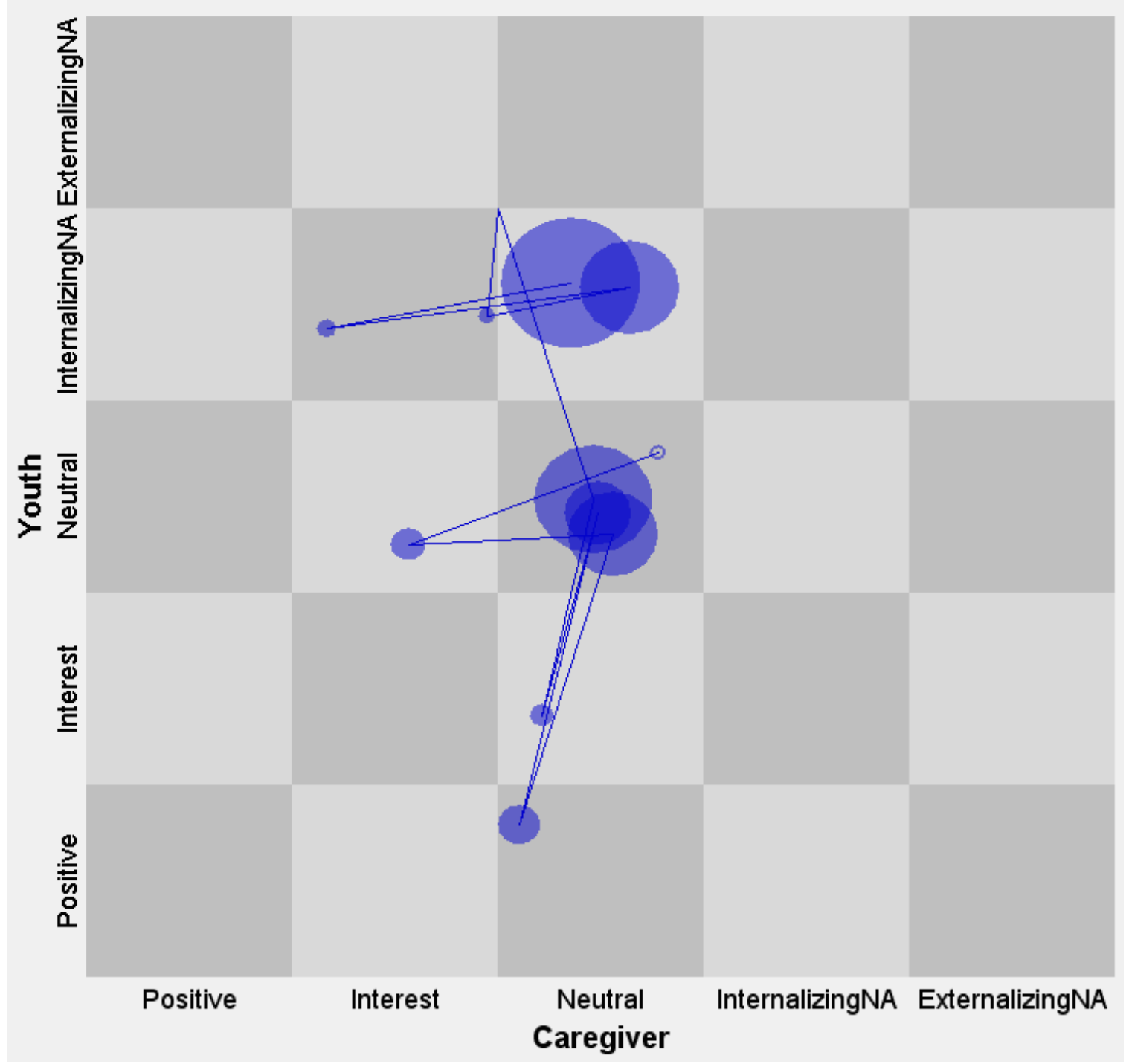


PSY2, Second Positive Discussion

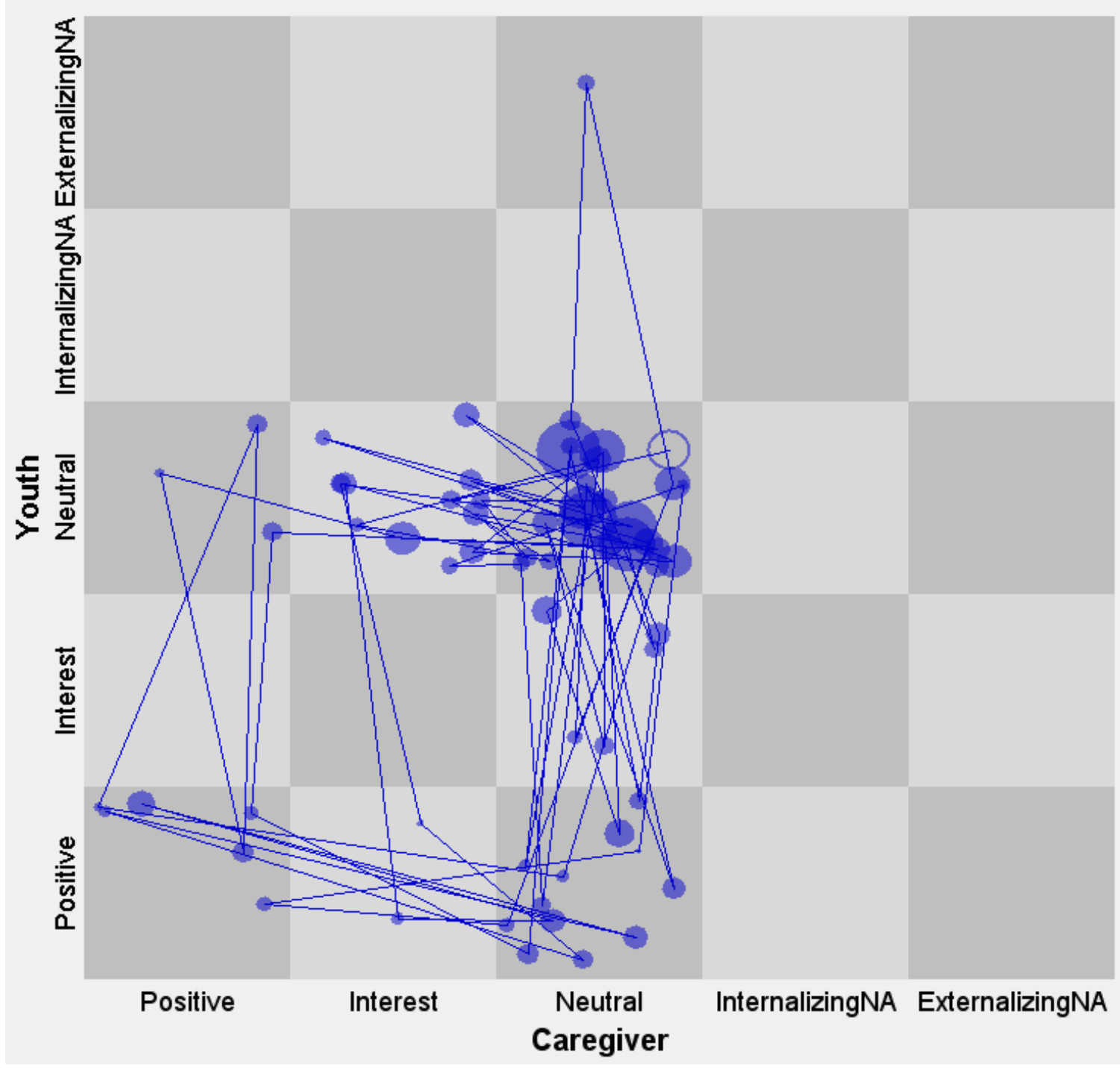


PSY2, Psychosis-Specific Conflict Discussion

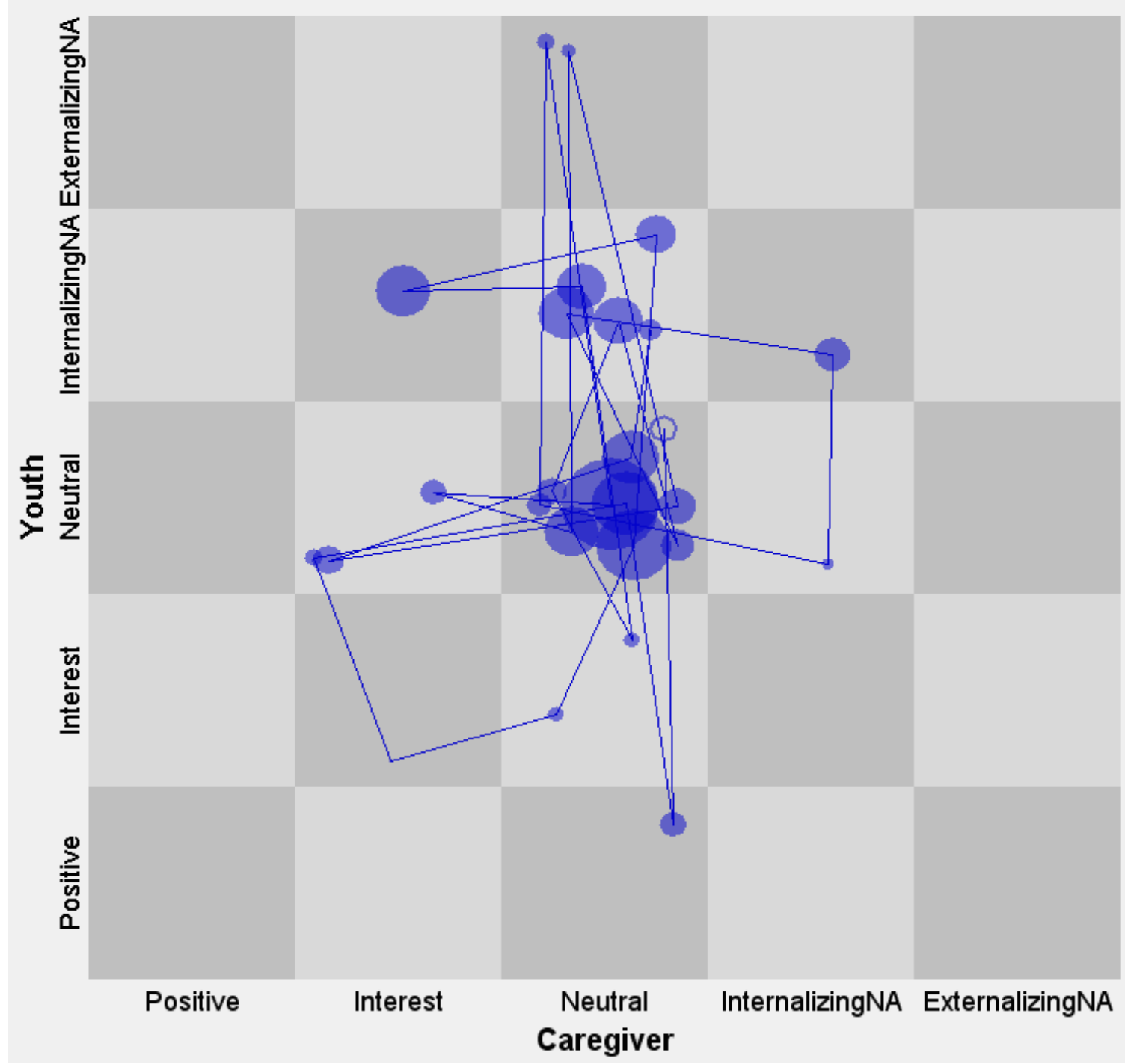


PSY2, Third Positive Discussion

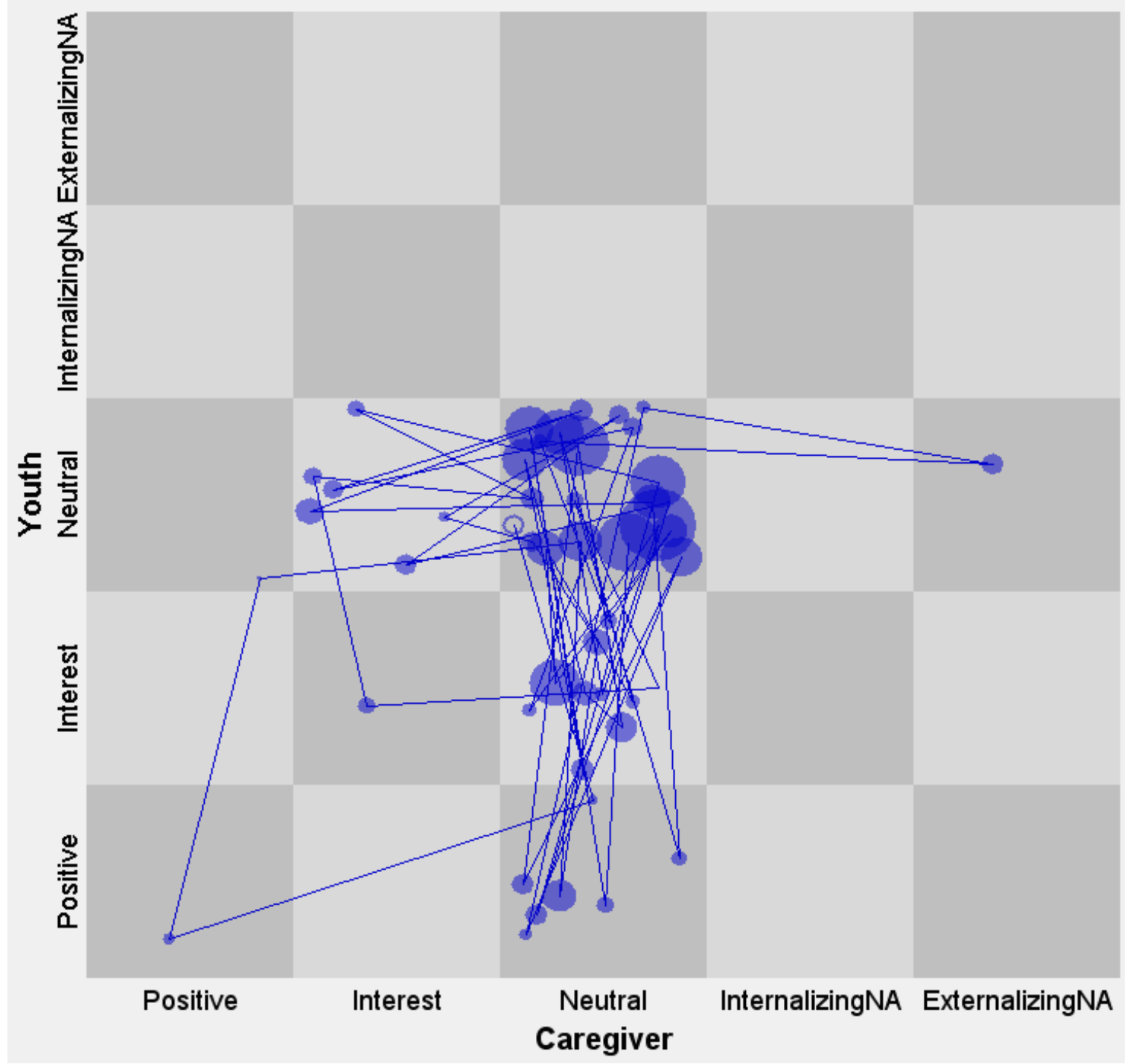


Family with an Individual with Psychosis 3 (PSY3)

PSY3, First Positive Discussion

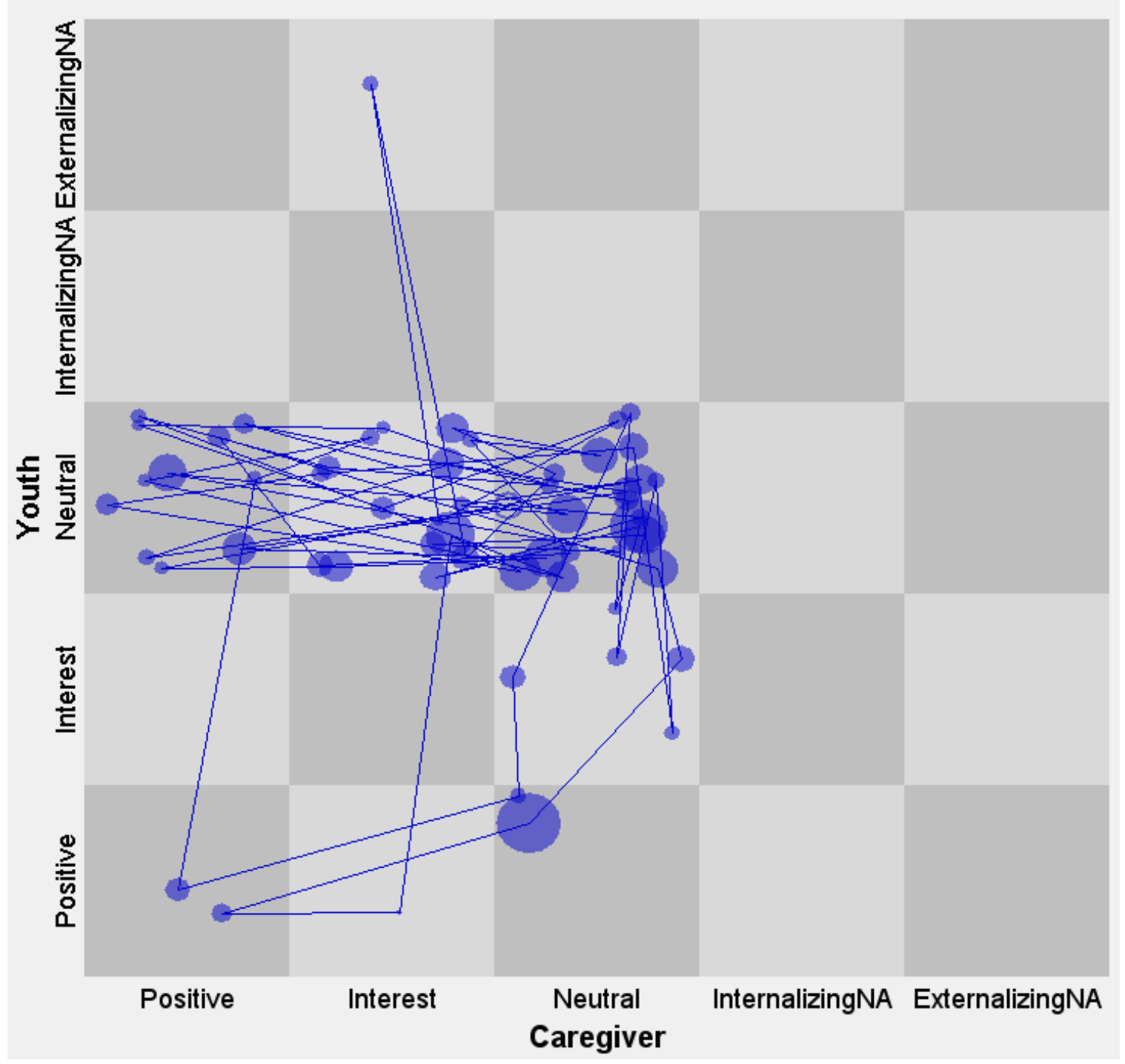


PSY3, Normative Conflict Discussion

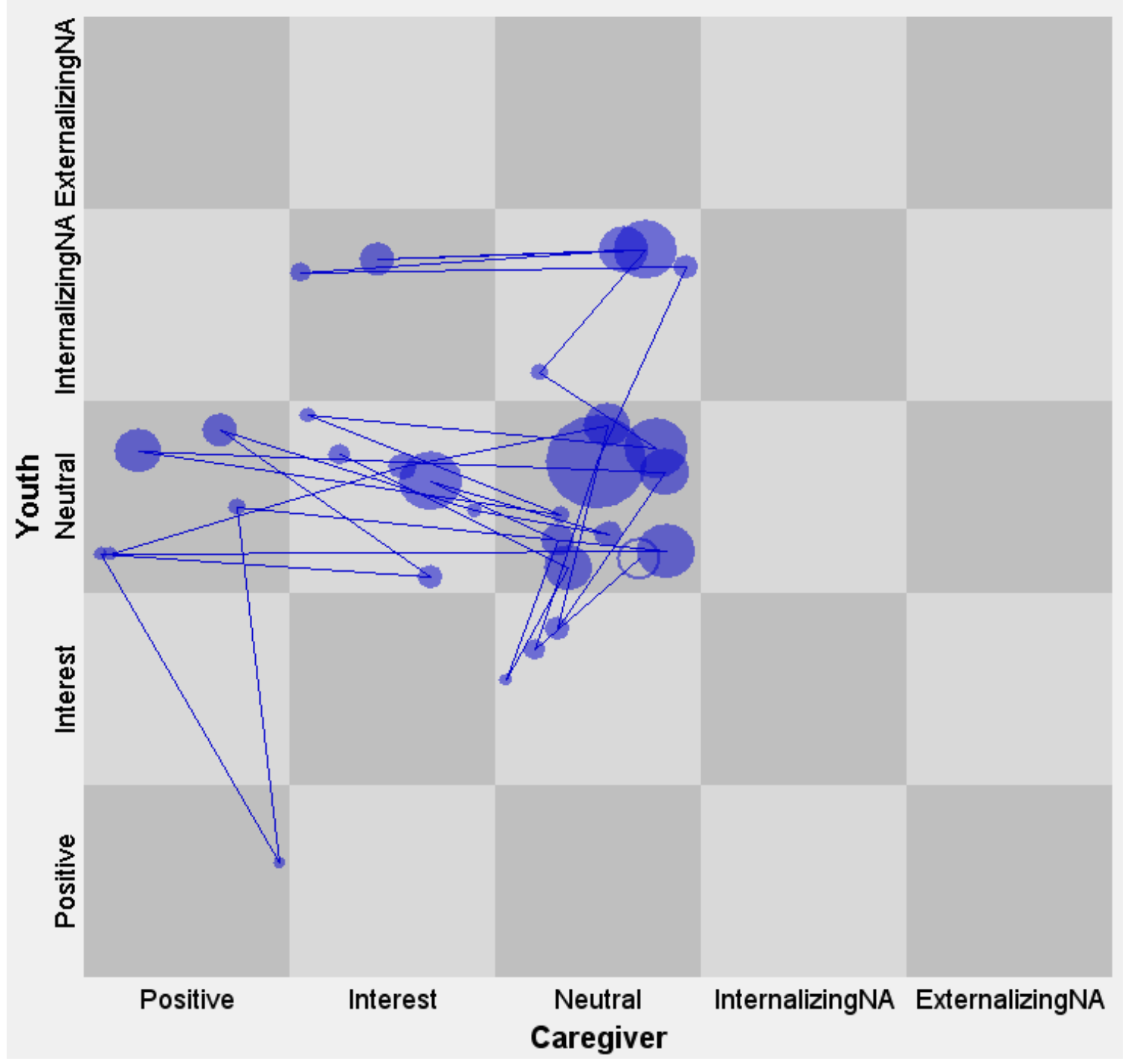


PSY3, Second Positive Discussion

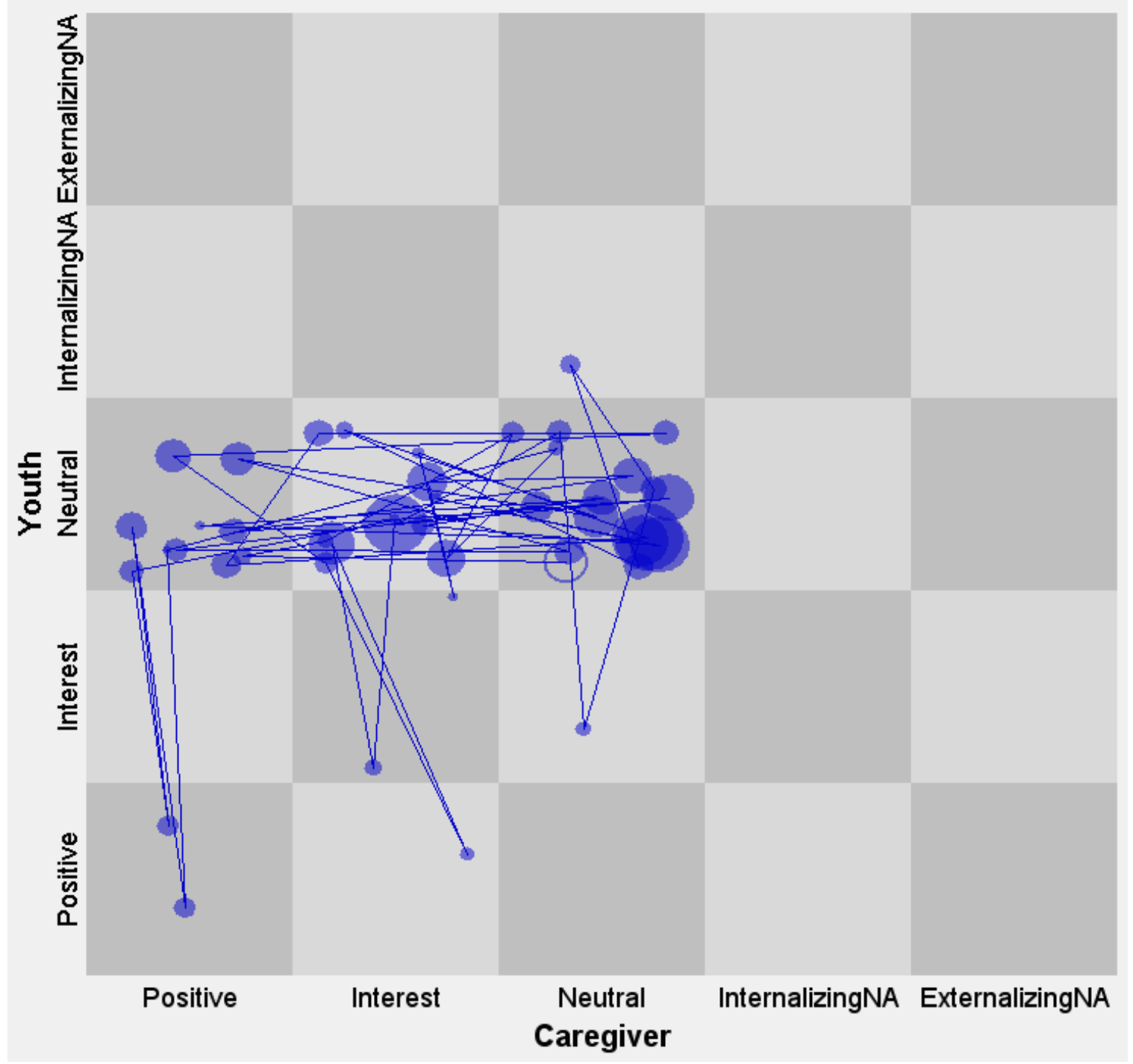


PSY3, Psychosis-Specific Conflict Discussion

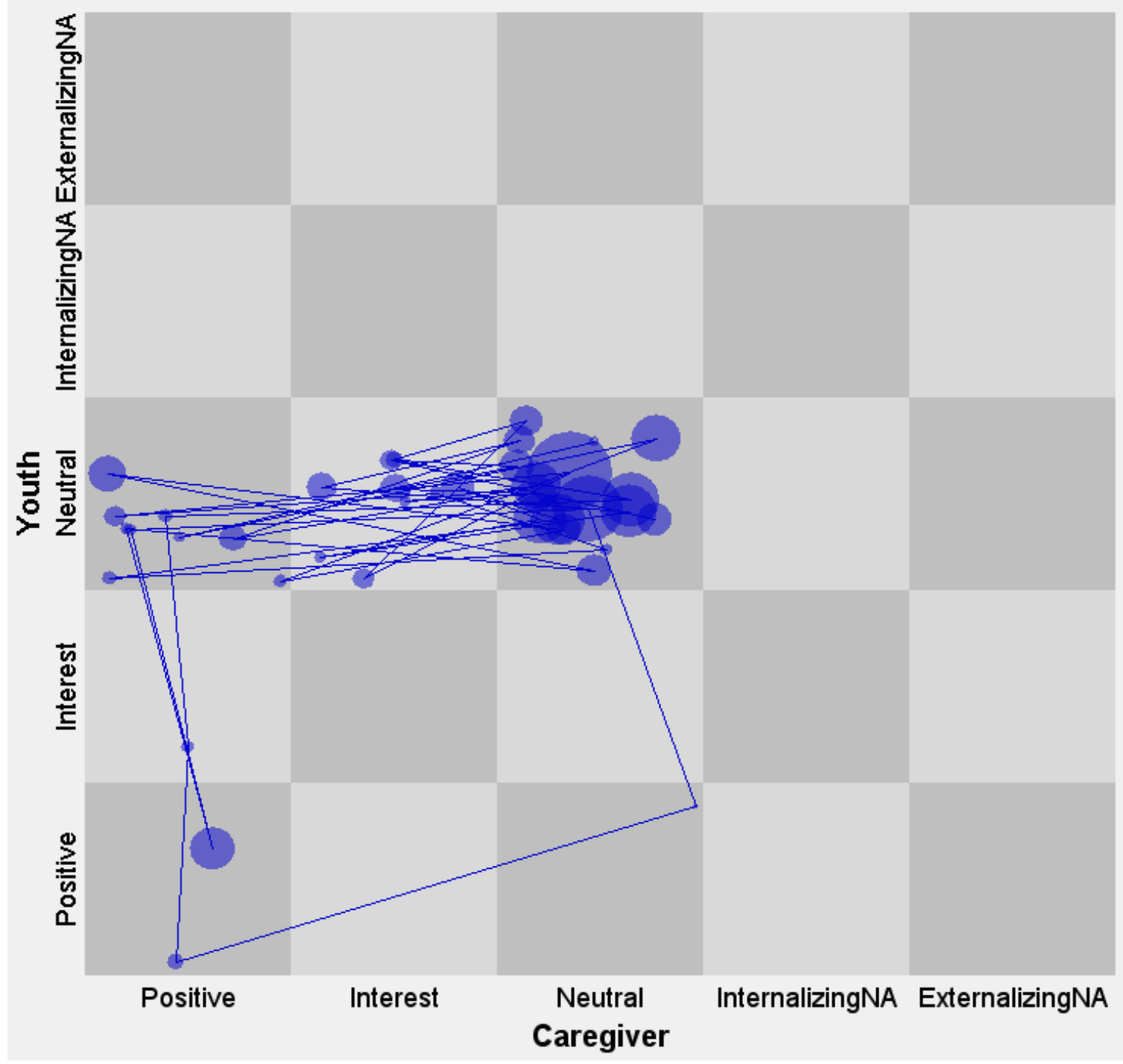


PSY3, Third Positive Discussion

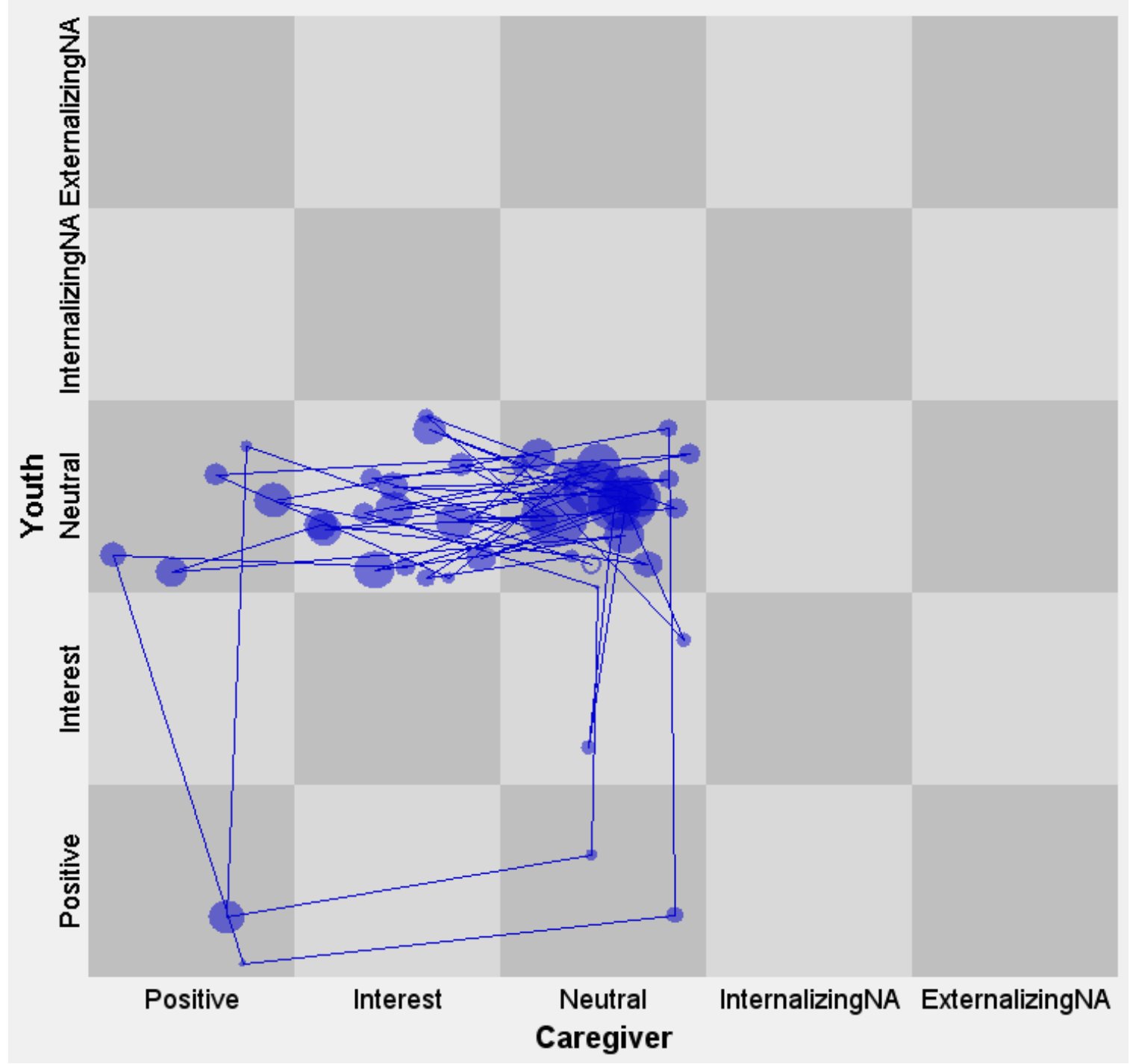


Family with an Individual with Psychosis 4 (PSY4)

PSY4, First Positive Discussion

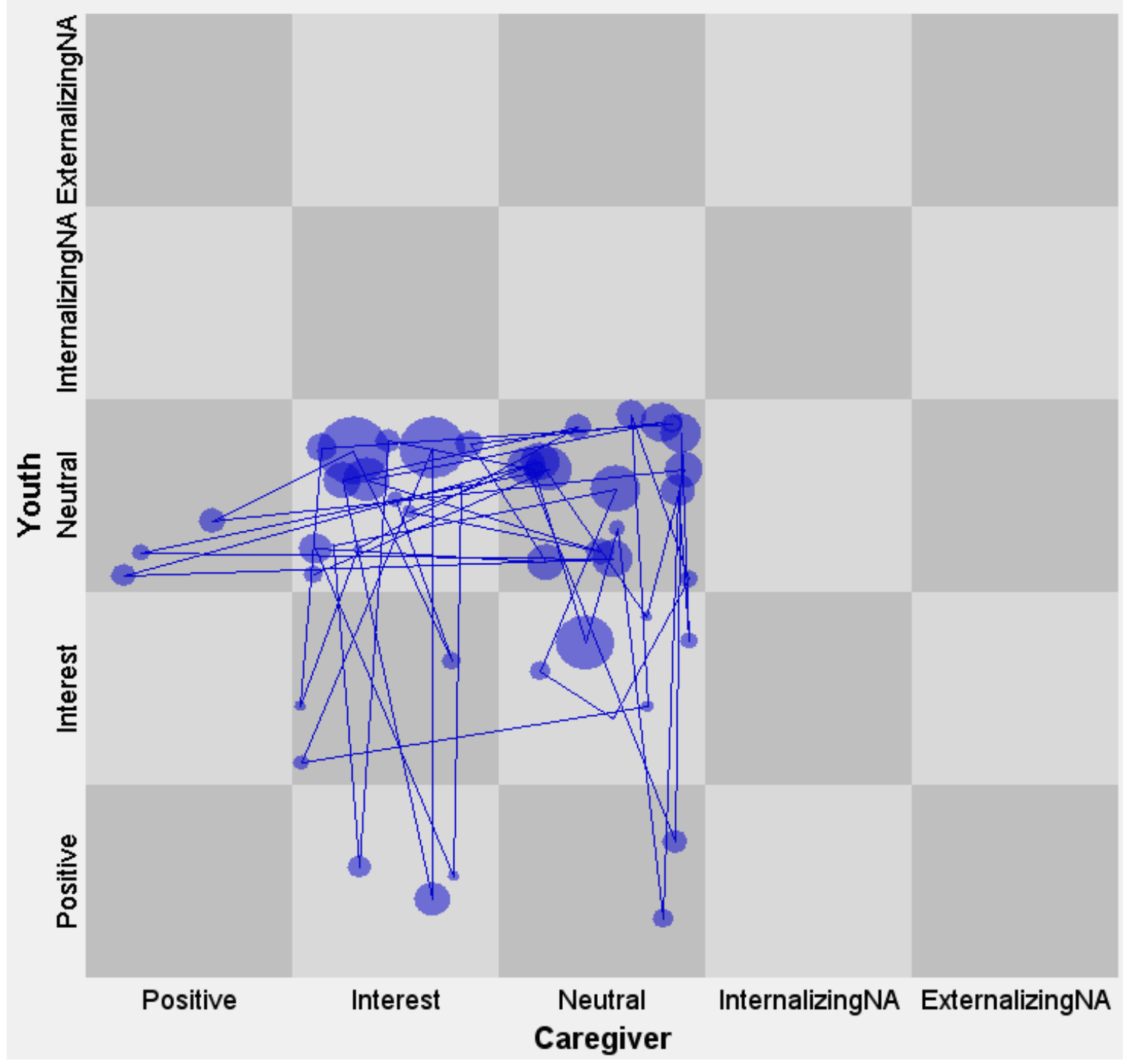


PSY4, Normative Conflict Discussion

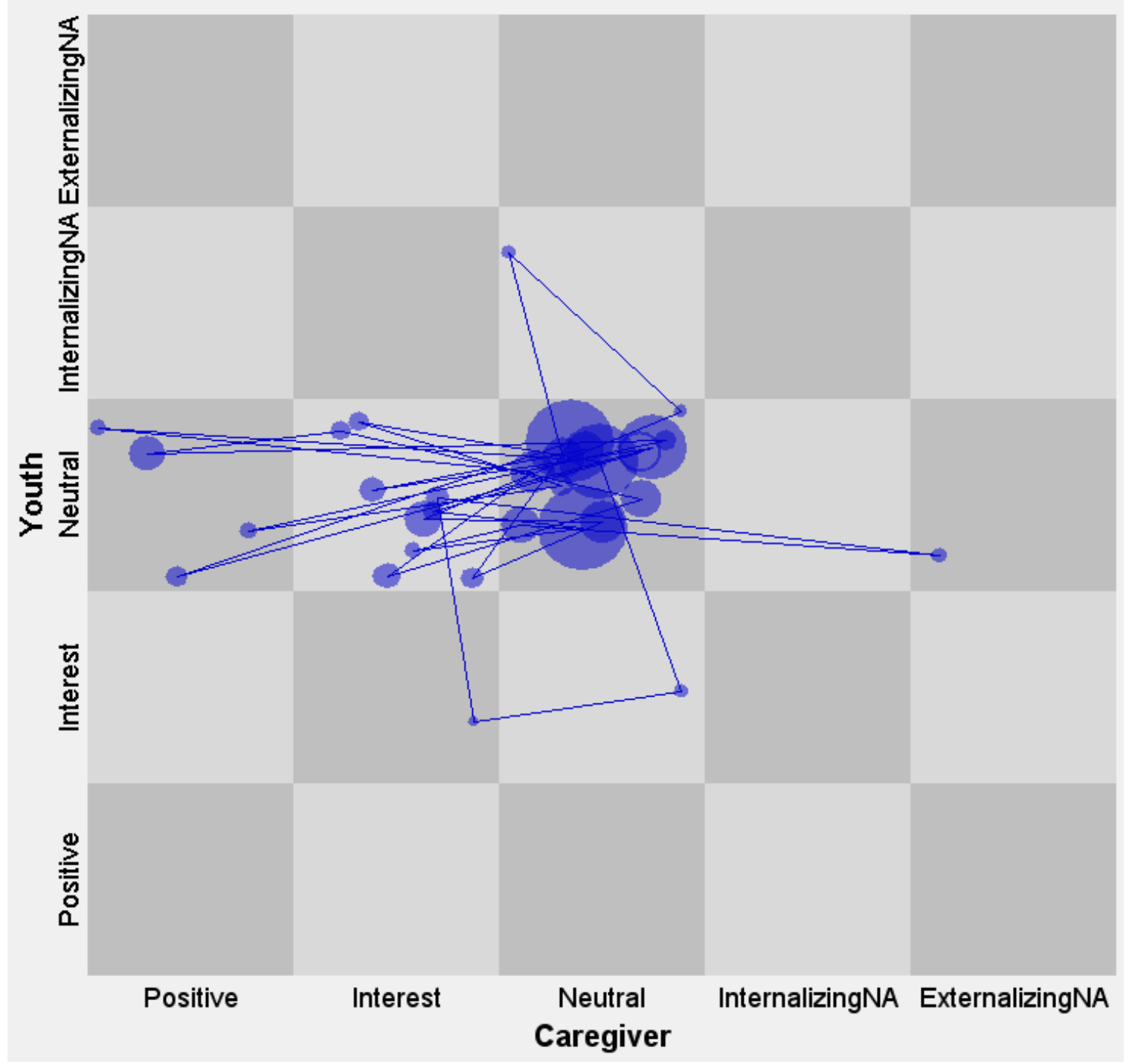


PSY4, Second Positive Discussion

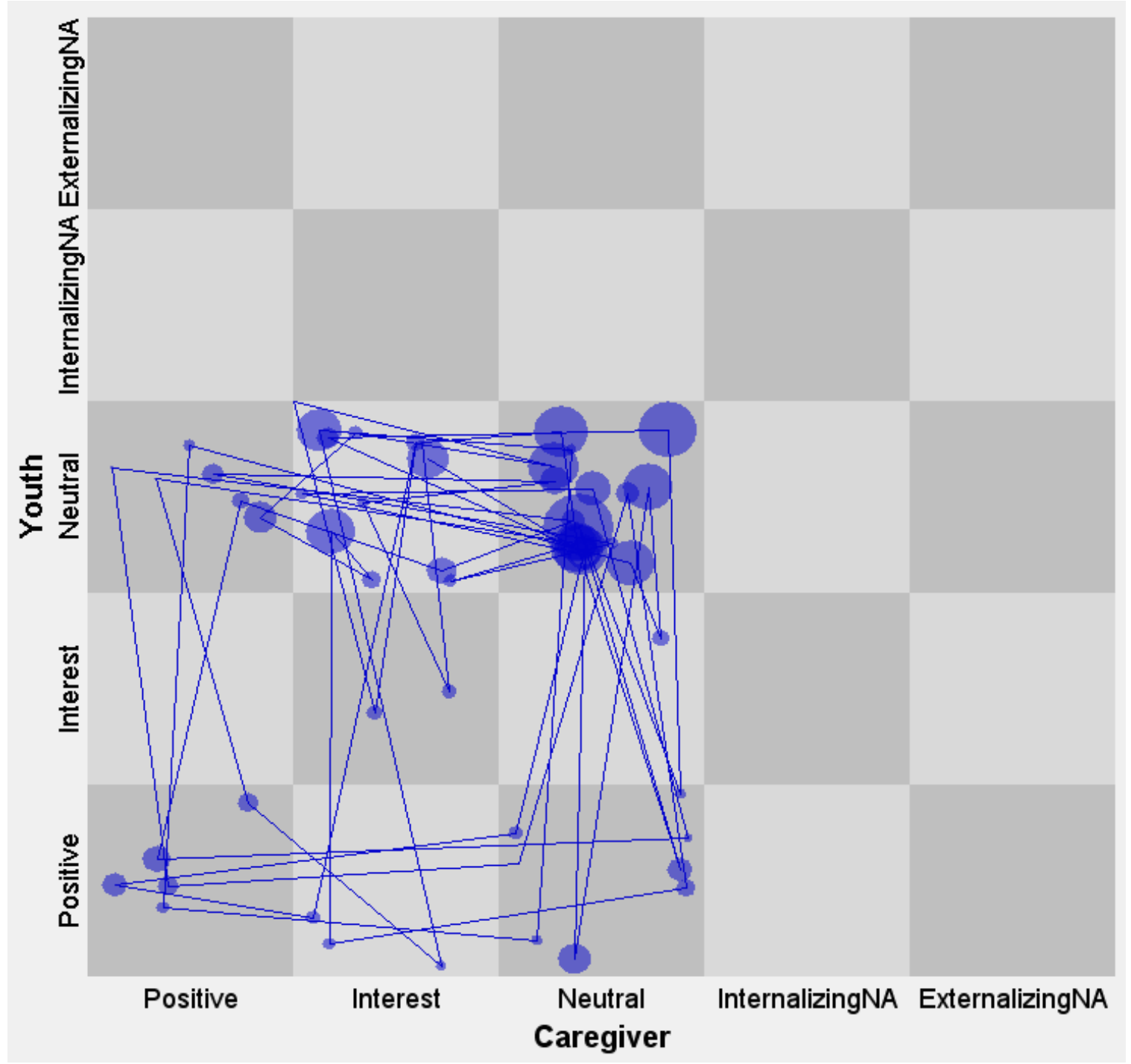


PSY4, Psychosis-Specific Conflict Discussion

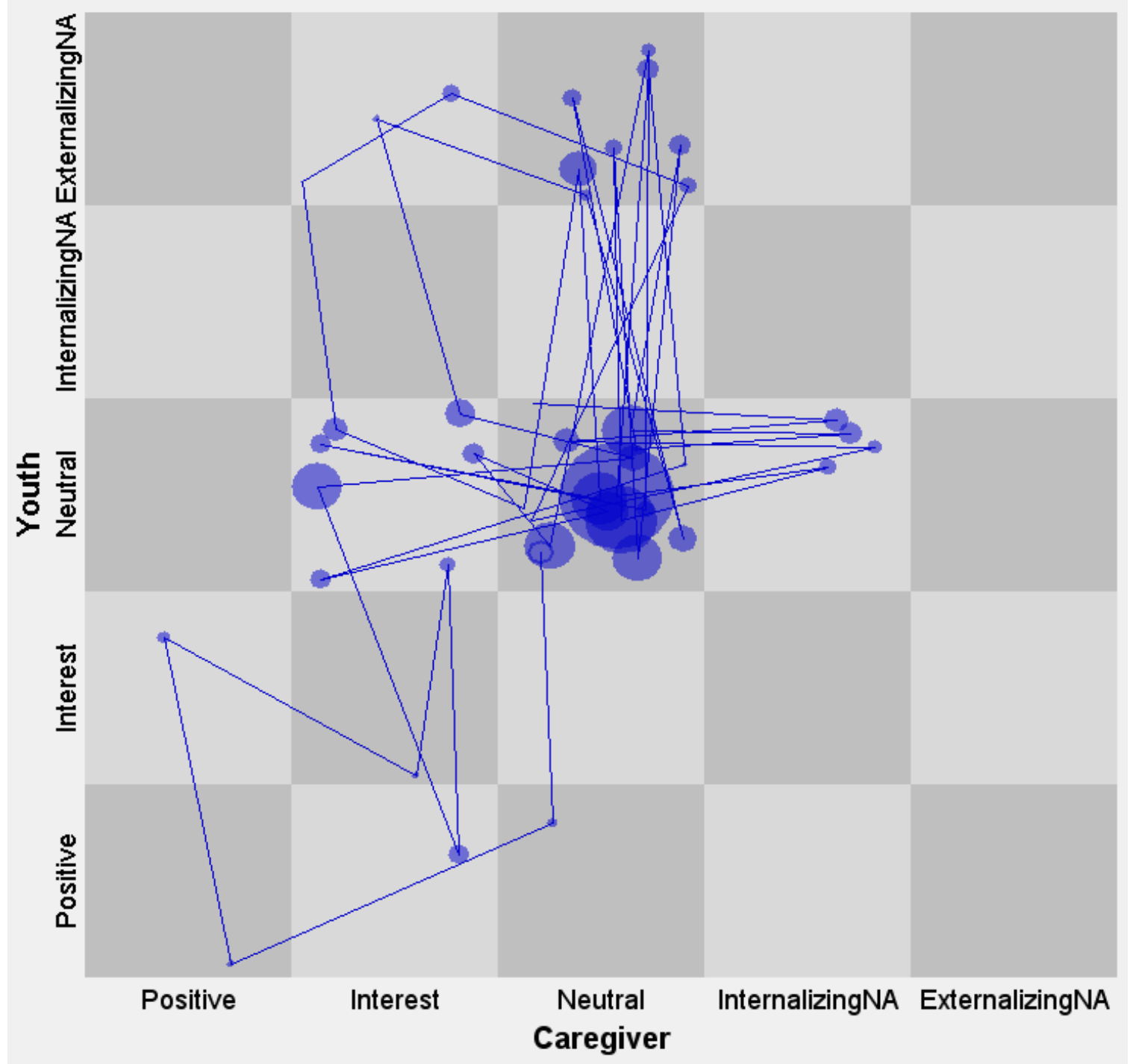


PSY4, Third Positive Discussion

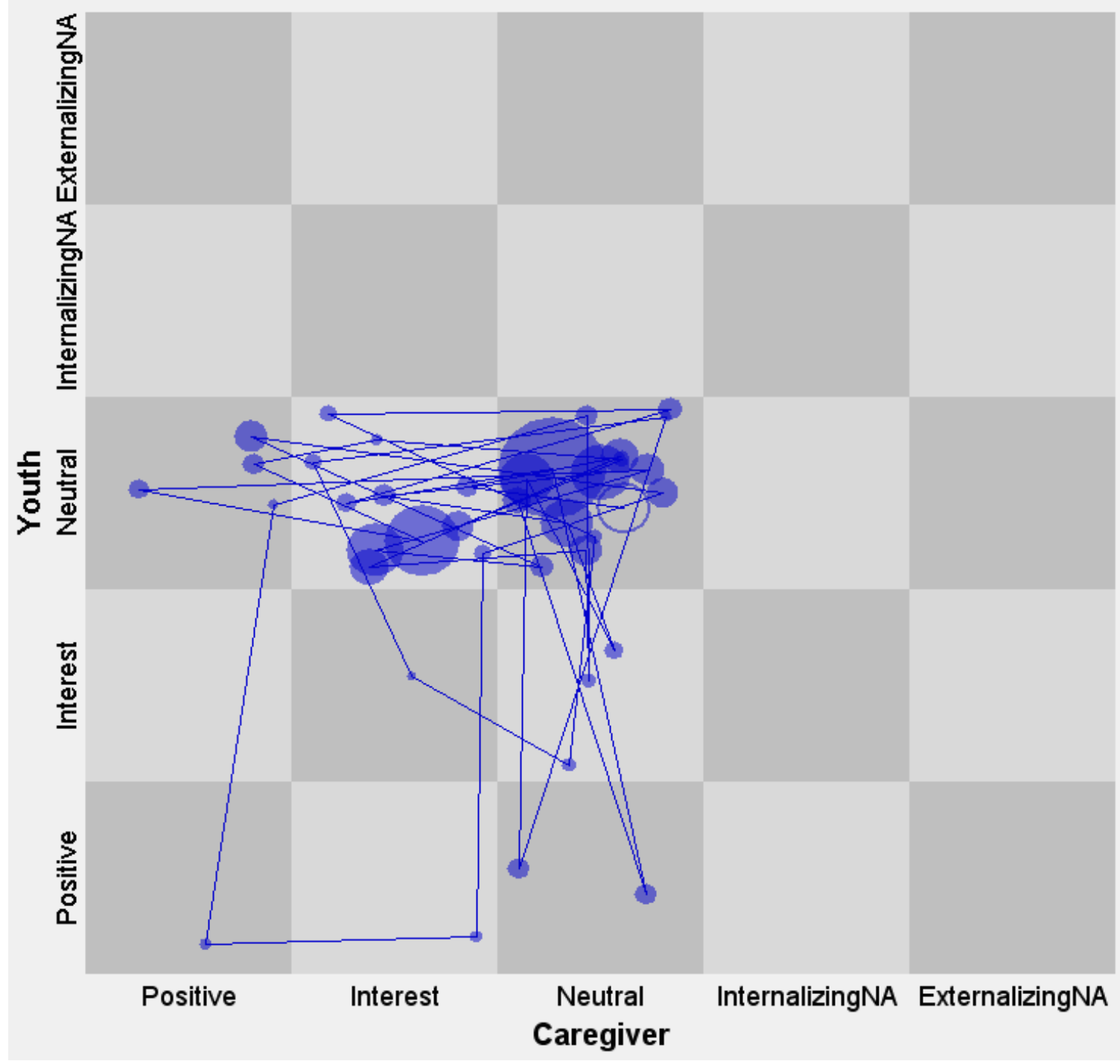


Family with an Individual with Psychosis 5 (PSY5)

PSY5, First Positive Discussion

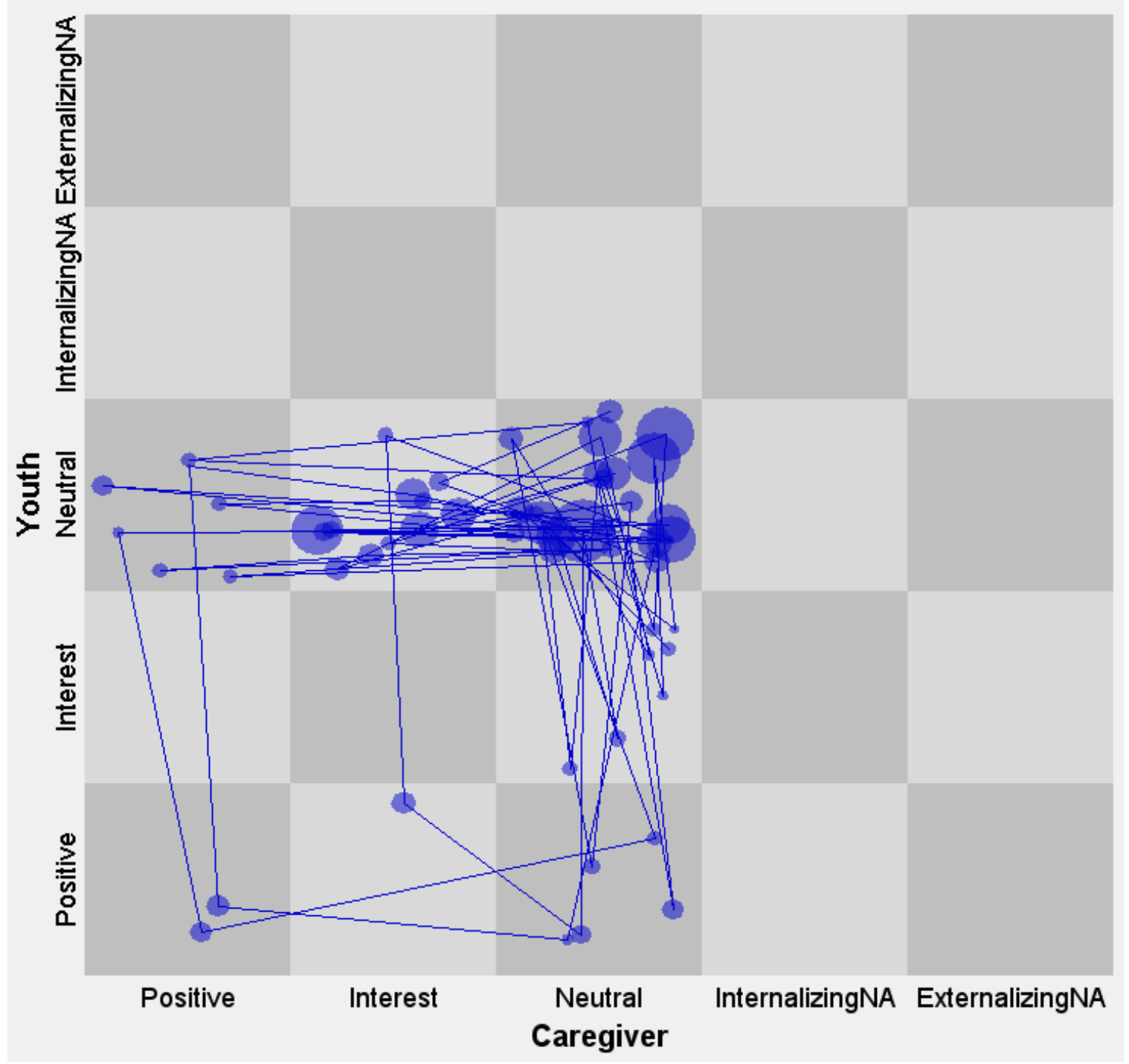


PSY5, Normative Conflict Discussion

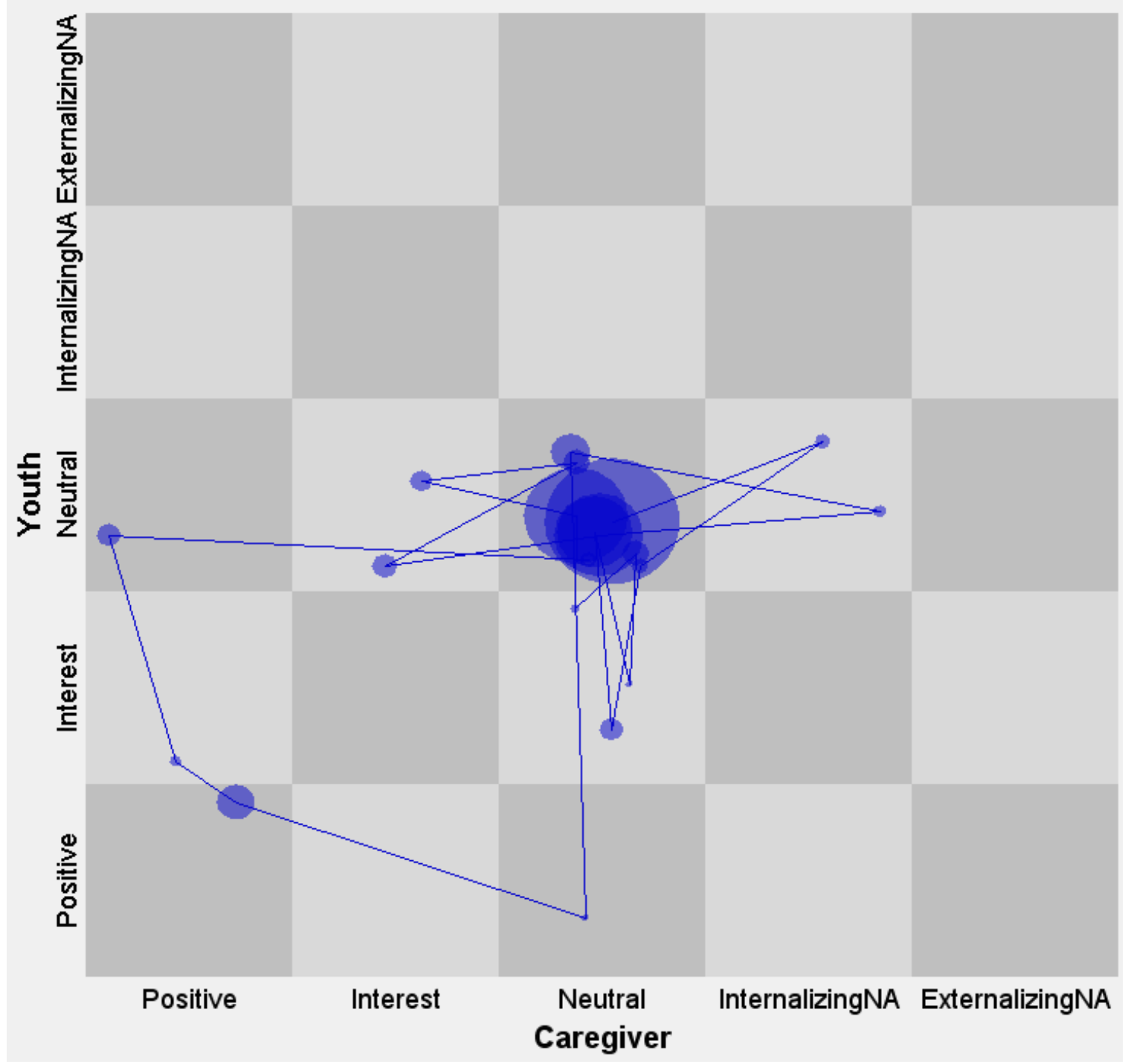


PSY5, Second Positive Discussion

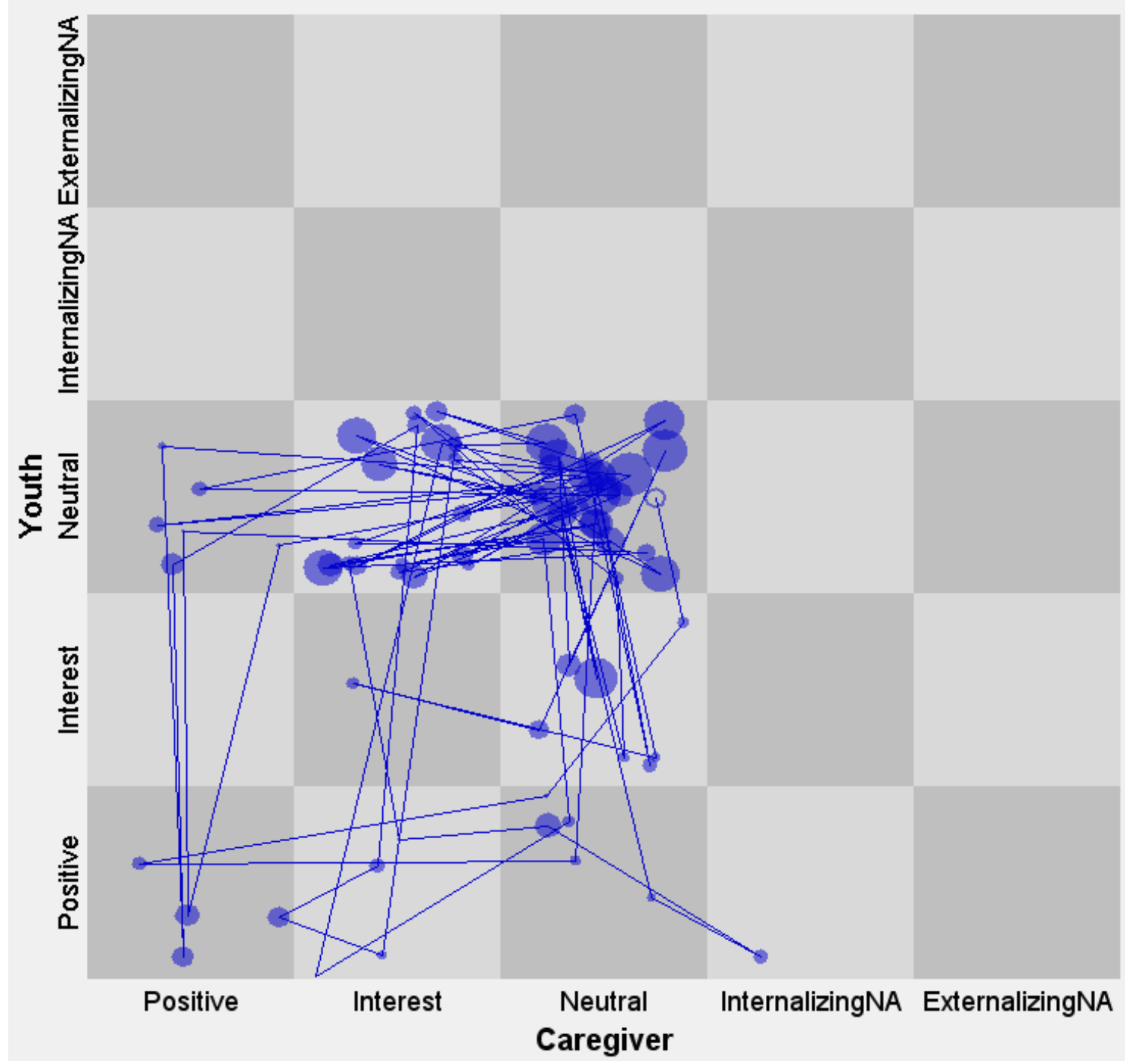


PSY5, Psychosis-Specific Conflict Discussion

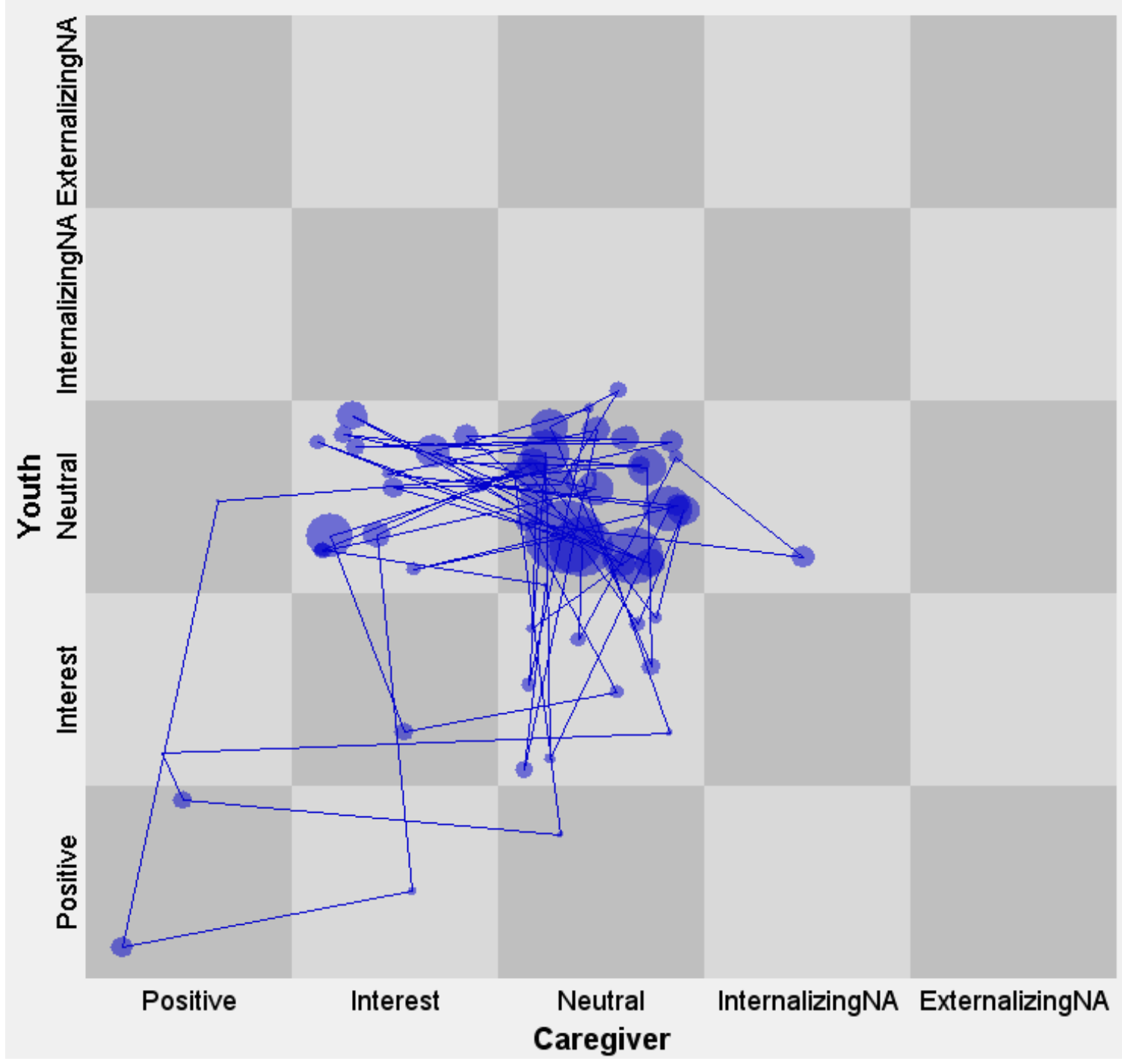


PSY5, Third Positive Discussion

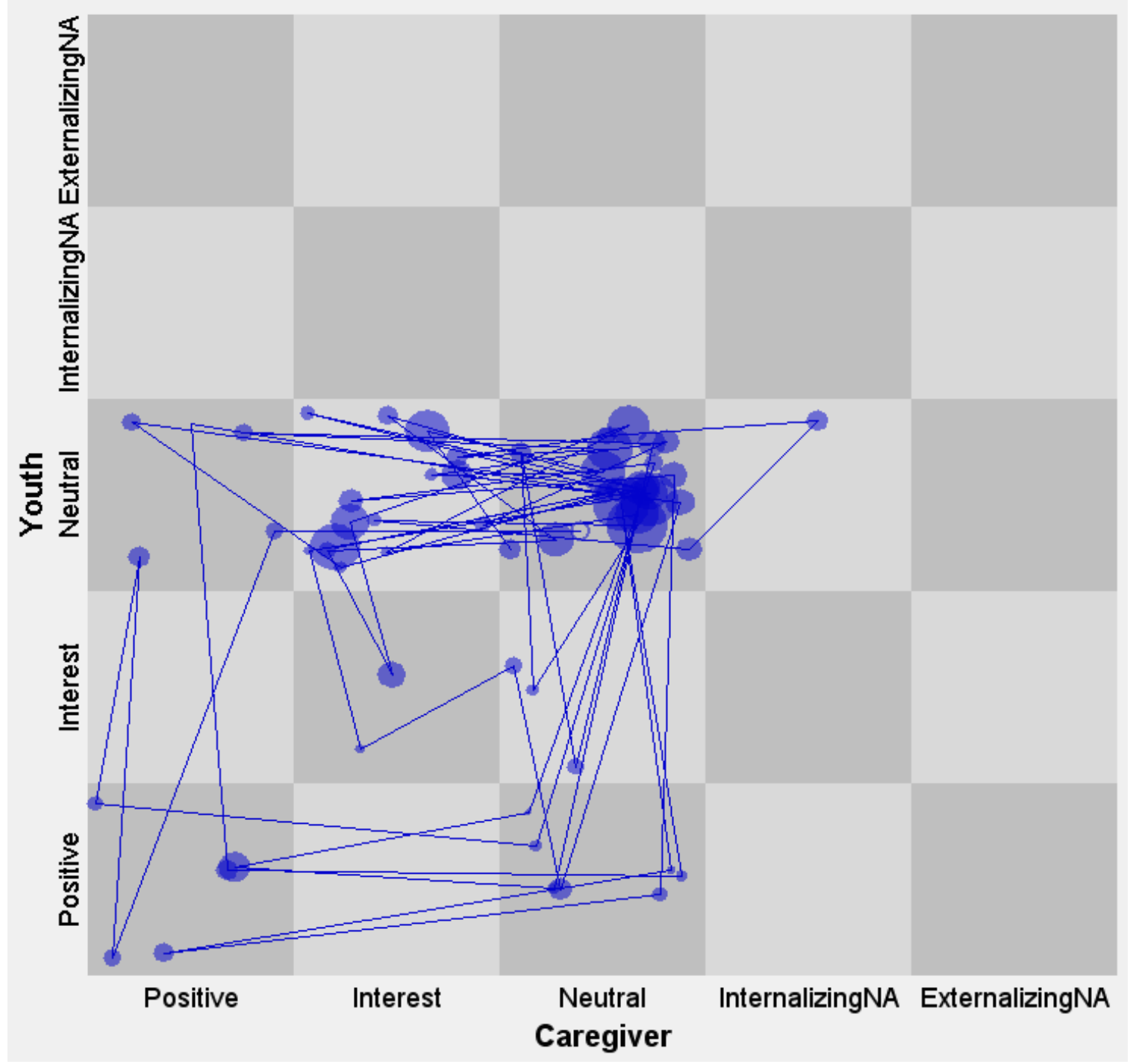

